\title{
ON REPRESENTATIONS DISTINGUISHED BY UNITARY GROUPS
}

\author{
by Brooke FEIGON, ERez LAPID, and OMer OFFEN
}

To the memory of Jonathan Rogawski

\begin{abstract}
Let $\mathrm{E} / \mathrm{F}$ be a quadratic extension of number fields. We study periods and regularized periods of cusp forms and Eisenstein series on $\mathrm{GL}_{n}\left(\mathbf{A}_{\mathrm{E}}\right)$ over a unitary group of a Hermitian form with respect to $\mathrm{E} / \mathrm{F}$. We provide factorization for these periods into locally defined functionals, express these factors in terms of suitably defined local periods and characterize global distinction. We also study in detail the analogous local question and analyze the space of invariant linear forms under a unitary group.
\end{abstract}

\section{CONTENTS}

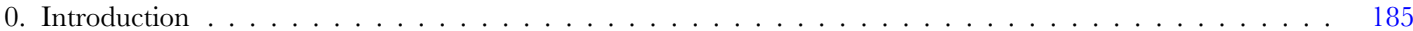

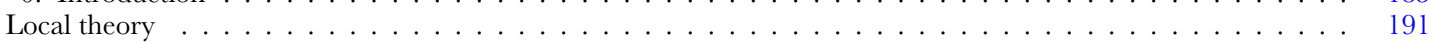

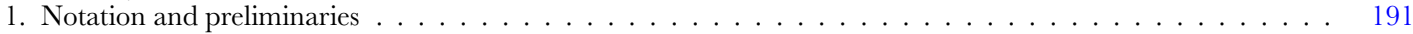

2. Bessel distributions . . . . . . . . . . . . . . . . . . . . . . . . . . . . . . . . . . . . 203

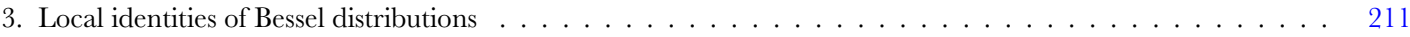

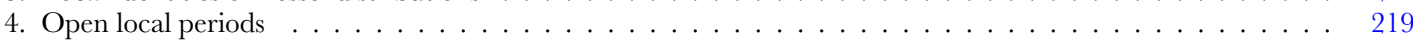

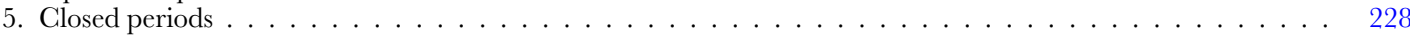

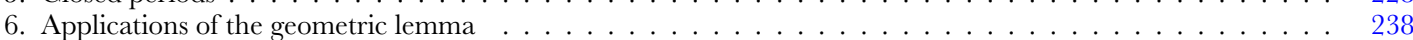

7. Local Bessel identities - the split case . . . . . . . . . . . . . . . . . . . . . . . . . . . 247

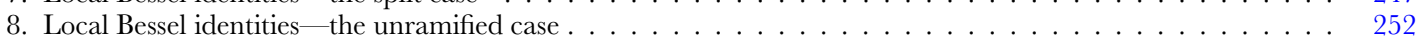

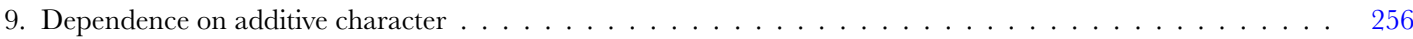

Global theory . . . . . . . . . . . . . . . . . . . . . . . . . . . . . . . . . . . . . . 263

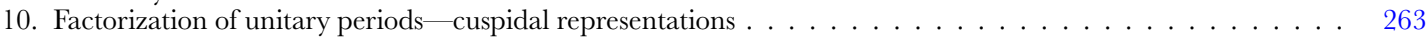

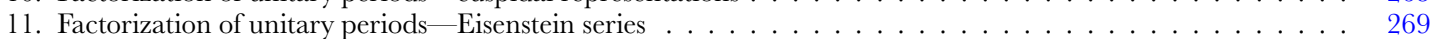

Local applications . . . . . . . . . . . . . . . . . . . . . . . . . . . . . . . . . . 280

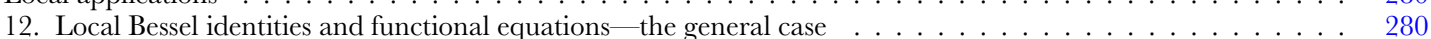

13. Multiplicity of unitary periods . . . . . . . . . . . . . . . . . . . . . . . . . . . . . 289

Acknowledgements . . . . . . . . . . . . . . . . . . . . . . . . . . . . . . . 298

Appendix A: Inner product for induced representations of $\mathrm{GL}_{n}$, by Erez Lapid and Omer Offen . . . . . . . . . . 298 Appendix B: Distinguished representations in the Archimedean case, by Avraham Aizenbud and Erez Lapid . . . 307 Appendix C: The relative trace formula, by Erez Lapid and Omer Offen . . . . . . . . . . . . . . . . . . . . . 313 Appendix D: Upper semicontinuity of multiplicity, by Erez Lapid and Omer Offen ～. . . . . . . . . . . . 317

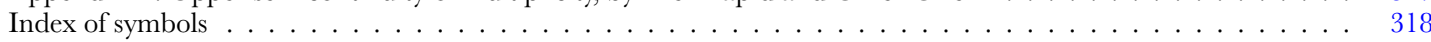

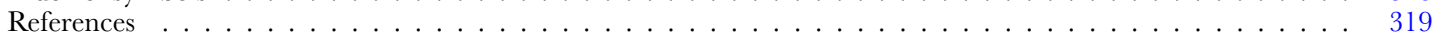

\section{Introduction}

Let $\mathrm{E} / \mathrm{F}$ be a quadratic extension of number fields with Galois involution $\tau$ and let $\mathbf{A}=\mathbf{A}_{\mathrm{F}}$ be the ring of adeles of F. Denote by $\eta: \mathrm{F}^{*} \backslash \mathbf{A}^{*} \rightarrow\{ \pm 1\}$ the quadratic character associated to E/F by class field theory. Let $\mathbf{G}^{\prime}=\mathrm{GL}_{n} / \mathrm{F}$ and let $\mathbf{G}$ be the restriction of scalars from $\mathrm{E}$ to $\mathrm{F}$ of $\mathrm{GL}_{n} / \mathrm{E}$. Let

$$
\mathbf{X}=\left\{g \in \mathbf{G}:{ }^{t} g^{\tau}=g\right\}
$$

The second and third named authors were partially supported by grants from the Israel Science Foundation. 
be the F-subvariety of Hermitian matrices in $\mathbf{G}$. Here ${ }^{t} g$ is the transpose of a matrix $g$. For $x \in \mathbf{X}(\mathrm{F})$ denote by

$$
\mathbf{G}^{\boldsymbol{x}}=\left\{g \in \mathbf{G}:{ }^{t} g^{\tau} x g=x\right\}
$$

the unitary group (defined over F) determined by $x$. The unitary period integral $\mathcal{P}_{x}(\phi)$ of a cusp form $\phi$ of $\mathbf{G}(\mathbf{A})\left(=\mathrm{GL}_{n}\left(\mathbf{A}_{\mathrm{E}}\right)\right)$ is defined by

$$
\mathcal{P}_{x}(\phi)=\int_{\mathbf{G}^{x}(\mathrm{~F}) \backslash \mathbf{G}^{x}(\mathbf{A})} \phi(h) d h .
$$

A cuspidal automorphic representation $\pi$ of $\mathbf{G}(\mathbf{A})$ is called $\mathrm{G}^{x}$-distinguished if there exists a cusp form $\phi$ in the space of $\pi$ such that $\mathcal{P}_{x}(\phi) \neq 0$. The study of unitary period integrals has seen much progress in recent years. In particular, Jacquet showed that an irreducible cuspidal automorphic representation $\pi$ of $\mathbf{G}(\mathbf{A})$ is in the image of quadratic base change bc if and only if $\pi$ is $\mathrm{G}^{x}$-distinguished for some $x \in \mathbf{X}(\mathrm{F})$ [Jac05a]. In fact, it suffices to take the quasi-split unitary group [Jac10]. In order to obtain these results, Jacquet, in the course of many years, invented and developed the relative trace formula on $\mathbf{G}(\mathbf{A})$ and together with his collaborators compared it to a Kuznetsov trace formula on $\mathbf{G}^{\prime}(\mathbf{A})$ (see [Ye88, Ye89, JY90, Jac92, JY92, Ye93, Ye94, Jac95, Ye95, JY96, Jac98, Ye98, JY99, Jac01, Jac02, Jac03a, Jac03b, Jac04b, Jac05a, Jac05b, Lap06, Jac 10]).

This comparison of trace formulas gives additional information about the period integrals. To describe it, we first recall that by Arthur-Clozel, an irreducible cuspidal automorphic representation $\pi$ of $\mathbf{G}(\mathbf{A})$ is in the image of quadratic base change if and only if $\pi$ is Galois invariant [AC89]. In this case, $\pi$ is the image of exactly two irreducible cuspidal representations of $\mathbf{G}^{\prime}(\mathbf{A})$ of the form $\pi^{\prime}, \pi^{\prime} \otimes \eta$. We say that $\mathrm{E} / \mathrm{F}$ splits at infinity if every Archimedean place of $\mathrm{F}$ splits in $\mathrm{E}$. When $n=3, x=e$ and $\mathrm{E} / \mathrm{F}$ splits at infinity, Jacquet showed in [Jac01, Theorem 1] that the period integral $\mathcal{P}_{x}$ is factorizable on $\pi$ and that its local components are governed by certain identities between Bessel distributions associated to the local components $\pi_{v}^{\prime}$ of $\pi^{\prime}$ and $\pi_{v}$ of $\pi$. The restriction to the case $n=3$ was made, since at the time, the necessary relative trace identity was only available in that case. Based on recent developments (mainly [Jac03b, Jac04b, Jac05a, Lap06]), the method and result of Jacquet can be generalized to any $n$ and $x$ (Theorem 10.2). We can also remove the assumption that $\mathrm{E} / \mathrm{F}$ splits at infinity, thanks to a recent result by Aizenbud and Gourevitch [AG] extending smooth transfer to the Archimedean case.

As pointed out in [Jac01], the factorization of the global unitary period is far from formal, since there is no multiplicity one for the local invariant functionals. For instance, for a quadratic extension of $p$-adic fields, any irreducible unramified principal series representation admits $2^{n-1}$ linearly independent invariant functionals with respect to a given unitary group. The failure of multiplicity one reflects the fact that locally, quadratic base change is not one-to-one, even up to a twist by $\eta$.

Along with the factorization we provide for any $x \in \mathbf{X}(\mathrm{F})$, a criterion for an irreducible, Galois-invariant, cuspidal automorphic representation $\pi=\operatorname{bc}\left(\pi^{\prime}\right)$ of $\mathbf{G}(\mathbf{A})$ to be 
$\mathrm{G}^{x}$-distinguished. Namely, $\pi$ is $\mathrm{G}^{x}$-distinguished except for purely local obstructions depending on the (finitely many) local components of $\pi^{\prime}$ (rather than $\pi$ ) at the places where $\mathrm{G}^{x}$ is not quasi-split. (We recall that $\pi_{v}^{\prime}$ is generally not determined by $\pi_{v}$, even up to a quadratic twist.) In the non-Archimedean case (for which non-quasi-split unitary groups exist only for $n$ even) the obstruction is that $\pi_{v}^{\prime} \simeq \pi_{v}^{\prime} \otimes \eta_{v}$. The Archimedean obstruction is a little more complicated, but it is still given explicitly in terms of $\pi_{v}^{\prime}$. More precisely, let $\tilde{\mathfrak{w}}^{\prime}\left(\pi_{v}^{\prime}\right)$ be the largest $k$ such that we can write $\pi_{v}^{\prime}=\delta^{\prime} \times \sigma^{\prime}$ where $\delta^{\prime}$ is a representation of $\mathrm{GL}_{2 k}\left(\mathrm{~F}_{v}\right)$ such that $\delta^{\prime} \simeq \delta^{\prime} \otimes \eta$ and $\times$ denotes parabolic induction. Then we have the following characterization.

Theorem 0.1. - Let $x$ be a Hermitian form of rank $n$ with respect to $\mathrm{E} / \mathrm{F}$ and let $\pi$ be an irreducible cuspidal representation of $\mathrm{GL}_{n}\left(\mathbf{A}_{\mathrm{E}}\right)$. Then $\pi$ is $\mathrm{G}^{x}$-distinguished if and only if there exists a cuspidal representation $\pi^{\prime}$ of $\mathrm{GL}_{n}\left(\mathbf{A}_{\mathrm{F}}\right)$ such that $\pi=\mathrm{bc}\left(\pi^{\prime}\right)$ (i.e., $\pi$ is Galois invariant) and $\tilde{\mathfrak{w}}^{\prime}\left(\pi_{v}^{\prime}\right)$ is not bigger than the Witt index of $x$ with respect to $\mathrm{E}_{v} / \mathrm{F}_{v}$ for all inert places $v$ of $\mathrm{F}$ over which $\mathrm{G}^{x}$ is not quasi-split.

In particular, with this criterion we may give examples, in the case where $\mathbf{G}^{\boldsymbol{x}}$ is not quasi-split, of cuspidal representations which are locally $\mathbf{G}^{x}\left(\mathrm{~F}_{v}\right)$-distinguished for all $v$, but not globally $\mathrm{G}^{x}$-distinguished (see Section 12.3). (The notion of local distinction is explained below.) At first glance this may look surprising since the special value of the L-function appearing in the period factorization is always non-vanishing. The point, however, is that the local functional dictated by the period integral may vanish, even if the corresponding local representation is abstractly distinguished.

The local factor of the global period $\mathcal{P}_{x}$ at a place $v$ is determined by the local component $\pi_{v}^{\prime}$ of the essentially unique $\pi^{\prime}$ that base changes to $\pi$. One of our main goals is to identify the local factors as local open periods (see below).

We recall that in many important cases of period integrals, the square of the absolute value of the period admits (or is expected to admit) a factorization into a product of locally defined positive semi-definite Hermitian forms on $\pi_{v}$ [Wal85, Ich08, II10]. Our context is different in that the factorization is already for $\mathcal{P}_{x}$ itself, but on the other hand, the local factors (even up to a scalar) are not determined by $\pi_{v}$ itself.

As it turns out, for the local analysis it is extremely useful to study the continuous part of the relative trace formula and to extend the factorization of unitary periods to Eisenstein series. Of course, the period integral may not converge, but it is possible to regularize it [LR03, LR01, JLR99]. On a formal level, the period of the Eisenstein series induced from a parabolic subgroup $\mathbf{P}$ unfolds to a sum over the $\mathbf{P}$-orbits in $\mathbf{X}$. The regularized period is the contribution of the open $\mathbf{P}$-orbit in $\mathbf{X}$ (whose F-points decompose to infinitely many $\mathbf{P}(\mathrm{F})$-orbits). The factorization of unitary periods of cusp forms, together with Fourier inversion, allows us to express the regularized period of an Eisenstein series as a finite sum of factorizable, invariant linear forms. The procedure is reminiscent of the stabilization of the trace formula of SL(2) [LL79]. A special case of this was 
carried out in [LR00] and [Off07]. We can analogously apply a Fourier transform locally to obtain open local periods and Bessel distributions. The open local periods are linear combinations of functionals considered in the generality of symmetric spaces by Blanc, Brylinsky, Carmona and Delorme [BD92, CD94, BD08]. We establish explicit local functional equations for the open local periods. This diagonalizes the vector-valued functional equations of [CD94] (see Theorem 12.4).

The question of global distinction admits a local analogue. Suppose that $\mathrm{E} / \mathrm{F}$ is a quadratic extension of local fields. By abuse of language, we call a linear form on a representation $\pi$ of $\mathbf{G}(\mathrm{F})=\mathrm{GL}_{n}(\mathrm{E})$ that is invariant under a unitary group, a local unitary period. We also say that $\pi$ is locally $G^{x}$-distinguished if a non-zero local unitary period with respect to $\mathbf{G}^{\boldsymbol{x}}(\mathrm{F})$ exists. We may ask the following questions.

- What are the distinguished representations $\pi$ of $\mathrm{GL}_{n}(\mathrm{E})$ ?

- What is the dimension of the space $\operatorname{Hom}_{\mathrm{G}^{x}}(\pi, \mathbf{C})$ of local unitary periods?

- Can one construct local unitary periods explicitly?

In the finite field case the answer has been known for a while. Namely, an irreducible representation $\pi$ of $\mathrm{GL}_{n}\left(\mathbf{F}_{q^{2}}\right)$ admits a non-zero functional invariant under the unitary group $\mathrm{U}_{n}\left(\mathbf{F}_{q}\right)$ if and only if $\pi$ is equivalent to its twist under the non-trivial element of $\operatorname{Gal}\left(\mathbf{F}_{q^{2}} / \mathbf{F}_{q}\right)$ and in this case this functional is unique up to a scalar [Gow84]. Moreover, the Galois-invariant representations of $\mathrm{GL}_{n}\left(\mathbf{F}_{q^{2}}\right)$ correspond bijectively, via character identities, to the irreducible representations of $\mathrm{GL}_{n}\left(\mathbf{F}_{q}\right)$ [Shi76].

Suppose that $\mathrm{F}$ is $p$-adic. The case $n=3$ is considered in [Jac01], where it is shown by a simple globalization argument, together with the necessary condition for distinction in the global case (both of which do not require any comparison of relative trace formulas) that a distinguished supercuspidal representation is Galois invariant. Conversely, using the relative trace formula, Jacquet shows that Galois-invariant supercuspidal representations are distinguished by any unitary group, and a non-zero local unitary period is

- unique up to scalar,

- can be obtained by integrating a matrix coefficient over $\mathbf{G}^{\boldsymbol{x}}(\mathrm{F})$,

- arises as a local component of a non-vanishing global unitary period integral.

Once again, using the aforementioned recent developments on the relative trace formula, the argument immediately extends to any $n$.

Jacquet further conjectured, in analogy with the global case, that in general, an irreducible representation is distinguished with respect to the quasi-split unitary group if and only if it is Galois invariant. Using the Geometric Lemma of Bernstein-Zelevinsky (but once again, without appealing to the comparison of relative trace formulas) we show the "only if" part of this conjecture (Theorem 6.1, which also applies in the non-quasisplit case). Using the local open periods that we define, we can prove in many cases the "if" part of the conjecture as well. Namely, we show that it holds if $\pi$ is either unitarizable or generic, and in the latter case the dimension of $\operatorname{Hom}_{\mathrm{G}^{x}}(\pi, \mathbf{C})$ is bounded from 
below by the number of equivalence classes of representations $\pi^{\prime}$ such that $\mathrm{bc}\left(\pi^{\prime}\right)=\pi$ up to a twist by $\eta$ (Proposition 13.14). Moreover, this bound is tight if $\pi$ is induced from pairwise inequivalent essentially square integrable representations (the regular case). On the other hand, in the non-regular case, a simple upper semicontinuity argument (see Appendix D) gives a sharper lower bound in general. One may speculate that the latter bound is actually tight.

Our results apply equally well in the non-quasi-split case. The only difference is in the case where there is a unique $\pi^{\prime}$ such that $\mathrm{bc}\left(\pi^{\prime}\right)=\pi$, in which case $\pi$ is not distinguished with respect to the non-quasi-split unitary group. We will call $\pi$ totally $\tau$-isotropic in this case. Equivalently, $\pi$ is totally $\tau$-isotropic if and only if $\pi$ is Galois invariant and the supercuspidal support of $\pi$ consists of $\sigma_{1} \otimes \cdots \otimes \sigma_{k}$ (up to permutation) where no $\sigma_{i}$ is Galois invariant. This can happen only if $n$ is even.

We summarize the main local results in the following

Theorem 0.2. - Let $\mathrm{E} / \mathrm{F}$ be a quadratic extension of $p$-adic fields, $x \in \mathbf{X}(\mathrm{F})$ and $\pi$ an irreducible representation of $\mathrm{GL}_{n}(\mathrm{E})$. Assume that $\pi$ is distinguished by $\mathrm{G}^{x}$. Then $\pi$ is Galois invariant. Conversely, assume that $\pi$ is Galois invariant. If $\pi$ is totally $\tau$-isotropic then

$$
\operatorname{dim} \operatorname{Hom}_{\mathrm{G}^{x}}(\pi, \mathbf{C})= \begin{cases}1 & \text { if } \mathrm{G}^{x} \text { is quasi-split, } \\ 0 & \text { otherwise }\end{cases}
$$

Otherwise,

(1) If $\pi$ is generic then $\pi$ is distinguished by $\mathrm{G}^{x}$. More precisely, assume that $\pi=\delta_{1} \times \cdots \times \delta_{k}$ where the $\delta_{i}$ 's are essentially square-integrable. Let $r$ be the number of $i$ 's such that $\delta_{i}$ is Galois invariant. (By assumption $r>0$.) Then

$(\mathbf{0 . 1})$

$$
\operatorname{dim} \operatorname{Hom}_{\mathrm{G}^{x}}(\pi, \mathbf{G}) \geq 2^{r-1}
$$

with equality if the Galois invariant $\delta_{i}$ 's are distinct.

(2) If $\pi$ is unitarizable (or more generally, $\pi$ is fully induced from ladder representations - see Definition 13.10) then $\pi$ is distinguished by $\mathrm{G}^{x}$.

We conjecture that in general equality holds in $(\mathbf{0 . 1})$.

In the Archimedean case, an analogue of Theorem 0.2 is proved in Appendix B.

There are other methods to study local unitary periods, especially for supercuspidal representations. For instance, Hakim-Mao, and in a more general context, HakimMurnaghan and Prasad, analyze unitary periods using the realization of a supercuspidal representation as induced from a compact open subgroup modulo the center [BK93], to reduce the problem to groups over finite fields [HM98, HM02b, Pra01]. In contrast, our method, which gives sharper results, is entirely different and exploits the full force of the relative trace formula and a global argument. 
Finally, let us describe the contents of the paper in more detail. The paper is divided into three parts and four appendices. The first part is purely local. After introducing relevant notation, we review some basic facts about representation theory of $\mathrm{GL}_{n}$, base change and Bessel distributions. This enables us to formulate a certain identity of appropriately normalized Bessel distributions for generic representations (Section 3). This identity will eventually be proved in full generality in Section 12 and it serves to normalize the local unitary period on the Whittaker model. The normalization of the Bessel distribution is based on the standard inner product on the Whittaker model. For inductive purposes it is useful to know that the inner product is compatible with parabolic induction, i.e., that the Jacquet integral is an isometry between an induced representation and its Whittaker model. This is shown in Appendix A.

In Section 4, we define and start to analyze the main local objects - the open local periods, following [LR00]. Eventually, they will show up as the local factors of global unitary periods. A complementary set of invariant functionals on an induced representation supported on closed orbits is defined in Section 5. They play a role in the exact characterization of global distinction. More precisely, they provide the missing link for the Archimedean analysis.

In Section 6, we prove that local distinction implies Galois invariance in the $p$-adic case (Theorem 6.1) answering positively the necessity part in Jacquet's conjecture on distinction. We also reduce the study of local unitary periods in the $p$-adic case to the case of representations which are subquotients of representations induced from a tensor product of unramified twists of a single Galois-invariant supercuspidal representation. The main tools are the geometric Lemma of Bernstein-Zelevinsky and a globalization argument which had been used by several authors (cf. [HM02a, Jac01]). The globalization uses a "soft" version of the relative trace formula and does not require any comparison. We also extend the formulation of Jacquet's conjecture on local distinction to include the non-quasi-split and the Archimedean cases.

In Section 7, we analyze the open periods in the split case and interpret them in terms of intertwining operators. Using Shahidi's expression for the local coefficients in the context of GL( $n)$ [Sha84, Sha90] we derive the local Bessel identities. The unramified case is considered in Section 8 where we use explicit computations of Y. Hironaka [Hir99] and the results of [Off09, Off07] to provide the Bessel identities at hand. The dependence of our results on the choice of the additive character is analyzed in Section 9. This is necessary in order to apply a global argument later on. Subsequently, in Appendix B by Aizenbud and Lapid we analyze the Archimedean case. We give a necessary condition (which is also conjectured to be sufficient) for distinction by a unitary group and an upper bound on the dimension of invariant forms. In particular, we show that a distinguished irreducible representation is Galois invariant.

In the second part of the paper we prove the main global results. In Section 10 we prove, following Jacquet, the factorization theorem for cuspidal representations (Theorem 10.2) and its corollaries for distinction. The factorization of unitary periods of Eisen- 
stein series after applying Fourier inversion is carried out in Section 11, following [LR00]. Along the way, we obtain identities of global Bessel distributions. The relative trace formula will be reviewed in Appendix C.

In the last part of the paper, we apply the results of the first two parts to prove our main local results. In Section 12, we prove the local functional equations for the open local periods and the existence of Bessel identities (Theorem 12.4). We also obtain an inductive description of the local factors of unitary periods in terms of the square-integrable data of the local components of the representation. Alongside, we obtain the precise local obstructions for global distinction by a given unitary group. In the final Section 13, we use the functional equations to give upper and lower bounds on the dimension of local unitary periods. In particular, we prove uniqueness of local unitary periods for squareintegrable representations, and more generally for a large class of representations named ladder representations. This class includes the general Speh representations and was studied in [LM]. In the regular case we compute the multiplicity of unitary periods precisely. We conclude with a summary of what is known and still missing about distinguished representations and the dimension of the space of linear forms invariant under a unitary group. Finally, in Appendix D we give an upper semicontinuity result for multiplicities. The argument, which is well known to experts, was kindly communicated to us by Joseph Bernstein, Akshay Venkatesh and Nolan Wallach.

For the convenience of the reader we append an index of symbols at the end of the paper.

\section{Local theory}

\section{Notation and preliminaries}

1.1. Groups and parabolic subgroups. - Throughout this part, let $\mathrm{F}$ be a local field of characteristic zero with normalized absolute value $|\cdot|_{\mathrm{F}}$. In the non-Archimedean case, let $\mathcal{O}_{\mathrm{F}}$ be the ring of integers of $\mathrm{F}, \varpi_{\mathrm{F}}$ a uniformizer and $q_{\mathrm{F}}$ the cardinality of the residue field. Let $\mathrm{E}$ be a quadratic étale algebra over $\mathrm{F}$, i.e., $\mathrm{E}$ is either a quadratic field extension of $\mathrm{F}$ (the inert case) or $\mathrm{E}=\mathrm{F} \oplus \mathrm{F}$ (the split case). Let $x \mapsto x^{\tau}$ denote the non-trivial $\mathrm{F}$ automorphism of $\mathrm{E}$. Thus, in the split case $(x, y)^{\tau}=(y, x), x, y \in \mathrm{F}$. Denote by $\operatorname{Tr}(x)=$ $x+x^{\tau}$ the trace map, $\operatorname{Nm}(x)=x x^{\tau}$ the norm map and $\eta$ the quadratic character attached to $\mathrm{E} / \mathrm{F}$ by class field theory. Thus $\eta$ is the unique non-trivial character of $\mathrm{Nm}\left(\mathrm{E}^{*}\right) \backslash \mathrm{F}^{*}$ in the inert case and $\eta$ is the trivial character of $\mathrm{F}^{*}$ in the split case. Throughout, we fix a non-trivial character $\psi^{\prime}$ of $\mathrm{F}$ and let $\psi=\psi^{\prime} \circ \mathrm{Tr}$. In the $p$-adic case, we say that $\psi^{\prime}$ is unramified if its conductor is $\mathcal{O}_{\mathrm{F}}$. We will usually suppress the dependence on $\psi^{\prime}$ from the notation.

We will often use a boldface letter, e.g. $\mathbf{Y}$, to denote an algebraic set defined over F. We will use a plain letter, e.g. Y, to denote the set of F-points of an algebraic set, i.e., $\mathrm{Y}=\mathbf{Y}(\mathrm{F})$. Let $\mathbf{G}^{\prime}=\mathbf{G}_{\boldsymbol{n}}^{\prime}$ denote the group $\mathrm{GL}_{n}$ considered as an algebraic group 
defined over $\mathrm{F}$ and let $\mathbf{G}=\mathbf{G}_{\boldsymbol{n}}=\operatorname{Res}_{\mathrm{E} / \mathrm{F}} \mathrm{GL}_{n}$ where Res denotes restriction of scalars. Thus, $\mathrm{G}^{\prime}=\mathrm{GL}_{n}(\mathrm{~F})$ and $\mathrm{G}=\mathrm{GL}_{n}(\mathrm{E})$. The group $\mathbf{G}^{\prime}$ is viewed as a subgroup of $\mathbf{G}$. Note that in the split case, $\mathbf{G}=\mathbf{G}^{\prime} \times \mathbf{G}^{\prime}$ and $\mathbf{G}^{\prime}$ embeds diagonally in $\mathbf{G}$. Let $e=\mathrm{I}_{n}$ be the identity element of $\mathrm{G}$. Denote by ${ }^{t} x$ the transpose of a matrix $x$. For a subgroup $\mathbf{Q}$ of $\mathbf{G}$, we denote by ${ }^{t} \mathbf{Q}$ its image under the transpose map.

Let

$$
\mathbf{X}=\mathbf{X}_{\boldsymbol{n}}=\left\{x \in \mathbf{G}:{ }^{t} x^{\tau}=x\right\}
$$

be the symmetric space of Hermitian matrices in $\mathbf{G}$ with the right $\mathbf{G}$-action given by

$$
x \bullet g={ }^{t} g^{\tau} x g, \quad g \in \mathbf{G}, x \in \mathbf{X} .
$$

We denote by $\mathrm{X} / \mathrm{G}$ the finite set of $\mathrm{G}$-orbits in $\mathrm{X}$. It is indexed by $\mathrm{F}^{*} / \mathrm{Nm} \mathrm{E}^{*}$ in the $p$-adic case and by the possible signatures in the Archimedean case. For an algebraic subgroup $\mathbf{Q}$ of $\mathbf{G}$ and any $x \in \mathrm{X}$, let $\mathbf{Q}^{\boldsymbol{x}}$ denote the stabilizer of $x$ in $\mathbf{Q}$. In particular, $\mathbf{G}^{\boldsymbol{x}}$ is the unitary group, defined over $\mathrm{F}$, associated with the Hermitian matrix $x$. For any $x \in \mathrm{X}$, we denote by $\mathfrak{w}(x)$ the Witt index of $x$, i.e., the F-rank of $\mathrm{G}^{x}$.

The standard maximal compact subgroup of $\mathrm{G}$ (resp., $\mathrm{G}^{\prime}$ ) is denoted by $\mathrm{K}$ (resp., $\mathrm{K}^{\prime}$ ). Thus,

$$
\begin{aligned}
& \mathrm{K}^{\prime}= \begin{cases}\mathrm{GL}_{n}\left(\mathcal{O}_{\mathrm{F}}\right) & \text { if } \mathrm{F} \text { is } p \text {-adic, } \\
\mathrm{O}(n) & \text { if } \mathrm{F}=\mathbf{R}, \\
\mathrm{U}(n) & \text { if } \mathrm{F}=\mathbf{C},\end{cases} \\
& \mathrm{K}= \begin{cases}\mathrm{K}^{\prime} \times \mathrm{K}^{\prime} & \text { if } \mathrm{E} / \mathrm{F} \text { is split } \\
\mathrm{GL}_{n}\left(\mathcal{O}_{\mathrm{E}}\right) & \text { if } \mathrm{E} / \mathrm{F} \text { is inert and } p \text {-adic, } \\
\mathrm{U}(n) & \text { if } \mathrm{E} / \mathrm{F}=\mathbf{G} / \mathbf{R}\end{cases}
\end{aligned}
$$

We shall fix some further notation and conventions pertaining to the group $\mathrm{G}$ with the inert case in mind; the corresponding notation for $\mathrm{G}^{\prime}$ will be appended by a prime. Denote by $\mathbf{Z}$ the center of $\mathbf{G}$. Let $\mathbf{P}_{\mathbf{0}}=\mathbf{M}_{\mathbf{0}} \mathbf{U}_{\mathbf{0}}$ be the standard Borel subgroup of $\mathbf{G}$ with its standard Levi decomposition, so that $\mathbf{P}_{\mathbf{0}}$ (resp., $\mathbf{M}_{\mathbf{0}}, \mathbf{U}_{\mathbf{0}}$ ) is the subgroup of upper triangular (resp., diagonal, upper unitriangular) matrices in $\mathbf{G}$. By a standard parabolic subgroup of $\mathbf{G}$ we mean one that contains $\mathbf{P}_{\mathbf{0}}$. Let $\kappa=\left(n_{1}, \ldots, n_{t}\right)$ be a composition of $n$, i.e., $n_{1}, \ldots, n_{t}$ are positive integers with $n=n_{1}+\cdots+n_{t}$. We denote by $\mathbf{P}_{\kappa}=\mathbf{M}_{\kappa} \mathbf{U}_{\kappa}$ the standard parabolic subgroup of $\mathbf{G}$ of type $\kappa$ with unipotent radical $\mathbf{U}_{\boldsymbol{\kappa}}$ and Levi part

$$
\mathbf{M}_{\kappa}=\left\{\operatorname{diag}\left(g_{1}, \ldots, g_{t}\right): g_{i} \in \mathbf{G}_{\boldsymbol{n}_{\boldsymbol{i}}}, i=1, \ldots, t\right\} .
$$

When $\kappa$ is clear from the context (and in particular, throughout this section) we simply write $\mathbf{M}=\mathbf{M}_{\kappa}, \mathbf{U}=\mathbf{U}_{\boldsymbol{\kappa}}$ and $\mathbf{P}=\mathbf{M U}$; similarly for $\mathbf{M}^{\prime}, \mathbf{U}^{\prime}, \mathbf{P}^{\prime}$. Let $\overleftarrow{\kappa}=\left(n_{t}, \ldots, n_{1}\right)$ be the reverse composition. In particular $\mathbf{P}_{\overleftarrow{\kappa}}$ is the standard parabolic subgroup which is 
conjugate to the opposite parabolic ${ }^{\boldsymbol{t}} \mathbf{P}$ of $\mathbf{P}$. For $\mathbf{P}=\mathbf{M U}$ as above, we write $\overleftarrow{\mathbf{P}}=\mathbf{P}_{\overleftarrow{\mathrm{\kappa}}}$ $\overleftarrow{\mathbf{M}}=\mathbf{M}_{\overleftarrow{\mathrm{k}}}$ and $\overleftarrow{\mathbf{U}}=\mathbf{U}_{\overleftarrow{\mathrm{\kappa}}}$

We also consider a composition $\gamma=\left(\gamma_{1}, \ldots, \gamma_{t}\right)$ of $\kappa$, i.e., $\gamma_{i}$ is a composition of $n_{i}$ for $i=1, \ldots, t$. We think of $\gamma$ as a composition of $n$ refining $\kappa$. When $\gamma$ is clear from the context we write $\mathbf{L}=\mathbf{M}_{\gamma} \subseteq \mathbf{M}$ and $\mathbf{L}^{\prime}=\mathbf{M}_{\gamma}^{\prime} \subseteq \mathbf{M}^{\prime}$. Usually, $\mathbf{Q}=\mathbf{L V}$ is the standard parabolic subgroup of $\mathbf{G}$ with Levi subgroup $\mathbf{L}$ and unipotent radical $\mathbf{V}$.

We identify the Weyl group $\mathrm{W}$ of $\mathrm{G}$ with the permutation matrices, or with the symmetric group $\mathrm{S}_{n}$ on the set $[1, n]$, where for $a, b \in \mathbf{R}$ such that $b-a \in \mathbf{N}$ we write

$$
[a, b]=\{a, a+1, \ldots, b\} .
$$

As a rule, we will use a superscript to refer to objects pertaining to a Levi subgroup of $\mathrm{G}$. For instance, we denote by $\Delta_{0}^{\mathrm{M}}$ the set of simple roots of $\mathrm{M}$ with respect to its Borel subgroup $\mathrm{M} \cap \mathrm{P}_{0}, \mathrm{~W}^{\mathrm{M}}$ the Weyl group of $\mathrm{M}$ (identified with a subgroup of $\mathrm{W}$ ) and $w_{0}^{\mathrm{M}}$ the longest element in $\mathrm{W}^{\mathrm{M}}$, i.e. such that $w_{0}^{\mathrm{M}} \alpha<0$ for all $\alpha \in \Delta_{0}^{\mathrm{M}}$. Set $w_{0}=w_{0}^{\mathrm{G}}$. Let $\mathrm{W}^{\mathrm{M}}(\mathrm{L})$ be the set of all right $\mathrm{W}^{\mathrm{L}}$-reduced elements $w \in \mathrm{W}^{\mathrm{M}}$ such that $w \mathrm{~L} w^{-1}$ is a standard Levi subgroup of $\mathrm{M}$ and let $w_{\mathrm{L}}^{\mathrm{M}}$ denote the longest element of $\mathrm{W}^{\mathrm{M}}(\mathrm{L})$. Set $\mathrm{W}(\mathrm{L})=\mathrm{W}^{\mathrm{G}}(\mathrm{L})$ and $w_{\mathrm{L}}=w_{\mathrm{L}}^{\mathrm{G}}$. Note that $w_{0}^{\mathrm{M}}=w_{\mathrm{M}_{0}}^{\mathrm{M}}$ and $w_{0}=w_{\mathrm{M}} w_{0}^{\mathrm{M}}$. Explicitly, for $\mathrm{M}=\mathrm{M}_{\kappa}$ we have

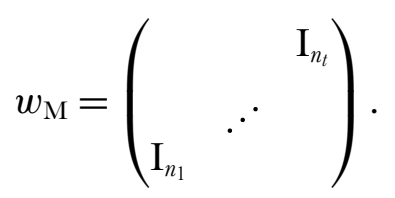

We view any $w \in \mathrm{W}(\mathrm{M})$ as the permutation of $[1, t]$ (also denoted by $w$ ) such that

$$
w \operatorname{diag}\left(g_{1}, \ldots, g_{t}\right) w^{-1}=\operatorname{diag}\left(g_{w^{-1}(1)}, \ldots, g_{w^{-1}(t)}\right) .
$$

Thus $w \mathrm{M} w^{-1}=\mathrm{M}_{w \kappa}$ where $w \kappa=\left(n_{w^{-1}(1)}, \ldots, n_{w^{-1}(t)}\right)$. In particular, $w_{\mathrm{M}} \kappa=\overleftarrow{\kappa}$ and $w_{\mathrm{M}}^{-1}=w_{\overleftarrow{\mathrm{M}}}$.

For a $\tau$-invariant subgroup $\mathbf{Q}$ of $\mathbf{G}$ denote by $\mathrm{X}^{*}(\mathbf{Q})$ the lattice of F-rational characters of $\mathbf{Q}$ and let $\delta_{\mathrm{Q}}$ be the modulus function of $\mathbf{Q}^{1}$ Let $\mathfrak{a}_{\mathrm{Q}}^{*}$ be the real vector space $\mathrm{X}^{*}(\mathbf{Q}) \bigotimes_{\mathbf{z}} \mathbf{R}$ and let $\mathfrak{a}_{\mathrm{Q}}$ be the dual space. If $\mathrm{N}$ is the Levi part of $\mathrm{Q}$ then we identify $\mathfrak{a}_{\mathrm{Q}}$ with $\mathfrak{a}_{\mathrm{N}}$ and with $\mathfrak{a}_{\mathrm{Z}(\mathrm{N})}$. We set $\mathfrak{a}_{0}=\mathfrak{a}_{\mathrm{M}_{0}}$ and $\mathfrak{a}_{0}^{*}=\mathfrak{a}_{\mathrm{M}_{0}}^{*}$. The Weyl group W acts naturally on $\mathfrak{a}_{0}^{*}$ and $\mathfrak{a}_{0}$. We identify $\mathfrak{a}_{0}^{*}$ and its dual space with $\mathbf{R}^{n}$ in the usual way. The $\mathrm{W}$-invariant pairing $\langle\cdot, \cdot\rangle: \mathfrak{a}_{0}^{*} \times \mathfrak{a}_{0} \rightarrow \mathbf{R}$ is the standard inner product on $\mathbf{R}^{n}$.

As before, let $\mathrm{L} \subseteq \mathrm{M}$ be standard Levi subgroups of $\mathrm{G}$. There is a natural embedding of $\mathfrak{a}_{M}$ into $\mathfrak{a}_{L}$. We denote by $\mathfrak{a}_{\mathrm{L}}^{\mathrm{M}}$ the orthogonal complement of $\mathfrak{a}_{\mathrm{M}}$ in $\mathfrak{a}_{\mathrm{L}}$ and use similar notation for the dual subspaces so that we also have $\mathfrak{a}_{\mathrm{L}}^{*}=\mathfrak{a}_{\mathrm{M}}^{*} \oplus\left(\mathfrak{a}_{\mathrm{L}}^{\mathrm{M}}\right)^{*}$. For every $\lambda \in \mathfrak{a}_{0}^{*}$ we denote by $\lambda_{\mathrm{L}}$, (resp. $\left.\lambda^{\mathrm{M}}, \lambda_{\mathrm{L}}^{\mathrm{M}}\right)$ its orthogonal projection to the space $\mathfrak{a}_{\mathrm{L}}^{*}$ (resp. $\left.\left(\mathfrak{a}_{0}^{\mathrm{M}}\right)^{*},\left(\mathfrak{a}_{\mathrm{L}}^{\mathrm{M}}\right)^{*}\right)$.

${ }^{1}$ The convention is that if $d q$ is a right Haar measure then $\delta_{\mathrm{Q}}(q)^{-1} d q$ is a left Haar measure. This is opposite to the convention of Bourbaki. 
In particular, for $M=M_{\kappa}$ we identify $\mathfrak{a}_{M}$ with $\mathbf{R}^{t}$ via

$$
\left(\lambda_{1}, \ldots, \lambda_{t}\right) \leftrightarrow(\overbrace{\lambda_{1}, \ldots, \lambda_{1}}^{n_{1}}, \ldots, \overbrace{\lambda_{t}, \ldots, \lambda_{t}}^{n_{t}}) \in \mathfrak{a}_{0} .
$$

We will identify $\mathfrak{a}_{\mathrm{M}^{\prime}}$ with $\mathfrak{a}_{\mathrm{M}}$.

For any real vector space $\mathfrak{a}$ we denote by $\mathfrak{a}_{\mathbf{C}}=\mathfrak{a} \bigotimes_{\mathbf{R}} \mathbf{G}$ its complexification. The function $\mathrm{H}: \mathrm{G} \rightarrow \mathfrak{a}_{0}$ is defined by

$$
e^{\langle\chi, \mathrm{H}(g)\rangle}=|\chi(m)|_{\mathrm{F}}, \quad \chi \in \mathrm{X}^{*}\left(\mathbf{M}_{\mathbf{0}}\right)
$$

via the Iwasawa decomposition $g=u m k, u \in \mathrm{U}_{0}, m \in \mathrm{M}_{0}, k \in \mathrm{K}$. Note that with our conventions we have $\mathrm{H}(g)=2 \mathrm{H}^{\prime}(g)$ for all $g \in \mathrm{G}^{\prime}$. We let

$$
\rho=\rho_{n}=\left(\frac{n-1}{2}, \frac{n-3}{2}, \ldots, \frac{1-n}{2}\right) \in \mathfrak{a}_{0}^{*}
$$

be the half-sum of the positive roots of $\mathrm{G}$ with respect to $\mathrm{P}_{0}$. Note that

$$
\delta_{\mathrm{Q} \cap \mathrm{M}}=e^{\left\langle 2 \rho_{\mathrm{L}}^{\mathrm{M}}, \mathrm{H}(\cdot)\right\rangle} .
$$

Denote by

$$
\left(\mathfrak{a}_{\mathrm{M}}^{*}\right)_{+}=\left\{\left(\lambda_{1}, \ldots, \lambda_{t}\right) \in \mathfrak{a}_{\mathrm{M}}^{*}: \lambda_{1}>\cdots>\lambda_{t}\right\}
$$

the positive Weyl chamber of $\mathfrak{a}_{\mathrm{M}}^{*}$.

Denote by $\mathbf{1}_{\Gamma}$ the characteristic function of a set $\Gamma$. For a group $Q$ let $1_{Q}$ denote the trivial character of $Q$. For every function $f$ on $Q$ and $x, y, z \in Q$, we set

$$
(\mathrm{L}(x) \mathrm{R}(y) f)(z)=f\left(x^{-1} z y\right) .
$$

For an affine variety $\mathbf{Y}$ over $\mathrm{F}$, we denote by $\mathcal{S}(\mathrm{Y})$ the space of Schwartz functions on $\mathrm{Y}=\mathbf{Y}(\mathrm{F})$. (We will only consider smooth varieties.) If $\mathrm{F}$ is $p$-adic, $\mathcal{S}(\mathrm{Y})$ is the space of locally constant compactly supported functions. If $\mathrm{F}$ is Archimedean, $\mathcal{S}(\mathrm{Y})$ consists of the restrictions to $\mathrm{Y}$ of Schwartz functions (in the usual sense) of the ambient affine space, with the usual topology. (This does not depend on the embedding.) If $\mathrm{H}$ is a group then $\mathcal{S}(\mathrm{H})$ is an algebra under the convolution $*$. Note that $\mathcal{S}(\mathrm{X})$ is a $\mathcal{S}(\mathrm{G})$-module via the convolution

$$
f * \Phi(x)=\int_{\mathrm{G}} f(g) \Phi(x \bullet g) d g .
$$

We denote by $\mathcal{S}^{*}(\mathrm{Y})$ the space of (Schwartz) distributions on $\mathrm{Y}$, i.e. the (continuous) linear forms on $\mathcal{S}(\mathrm{Y})$. 
1.2. Measures. - Recall that for any non-singular algebraic variety $\mathbf{V}$ over $\mathbf{F}$ of dimension $d$ with a gauge form $\boldsymbol{\omega}_{\mathbf{v}}$, the Tamagawa measure on $\mathrm{V}$ is defined by transferring the "standard" Haar measure on $\mathrm{F}^{d}$ to $\mathrm{V}$ using $\boldsymbol{\omega}_{\mathbf{v}}$. (See [Wei82]; the varieties that we consider will always be groups or homogeneous spaces and the gauge forms will be non-zero invariant differential forms.) For global reasons, we will multiply this measure by $\lambda_{\mathrm{V}} c\left(\psi^{\prime}\right)^{d / 2}$ where $\lambda_{\mathrm{V}}$ is a local factor described below and

$$
c\left(\psi^{\prime}\right)= \begin{cases}q^{m} & \text { if } \mathrm{F} \text { is non-Archimedean and } \\ & \text { the conductor of } \psi^{\prime} \text { is } \varpi^{m} \mathcal{O}_{\mathrm{F}}, \\ |a|_{\mathrm{F}} & \text { if } \mathrm{F} \text { is Archimedean and } \psi^{\prime}(x)=e^{2 \pi \mathrm{i} \operatorname{Tr}_{\mathrm{F} / \mathbf{R}} x a} .\end{cases}
$$

As a rule we take

$$
\boldsymbol{\omega}_{\operatorname{Res} / \mathrm{F}} \mathbf{v}=p^{*}\left(\boldsymbol{\omega}_{\mathbf{V}}\right)
$$

where $p^{*}$ is defined in [Wei82, p. 22]. (In the split case this becomes the product form.)

The invariant differential forms are chosen (up to a sign) as follows. We take $\boldsymbol{\omega}_{\mathbf{U}^{\prime}}=$ $\prod d u_{i, j}$ where the product ranges over the non-constant coordinates of $\mathbf{U}^{\prime}, \boldsymbol{\omega}_{\mathbf{G}^{\prime}}=\frac{\prod d g_{i, j}}{\operatorname{det} g^{n}}$, $\boldsymbol{\omega}_{\mathbf{M}^{\prime}}=\prod_{i=1}^{t} \boldsymbol{\omega}_{\mathbf{G}_{\boldsymbol{n}_{\boldsymbol{i}}}^{\prime}}$ and $\boldsymbol{\omega}_{\mathbf{P}^{\prime}}$ which matches $\boldsymbol{\omega}_{\mathbf{M}^{\prime}}$ and $\boldsymbol{\omega}_{\mathbf{U}^{\prime}}$ in the sense of [Wei82, p. 24]. The forms on $\mathbf{U}, \mathbf{G}, \mathbf{M}, \mathbf{P}$ are taken by the convention above. We also take $\boldsymbol{\omega}_{\mathbf{X}}=\frac{\prod_{i \leq j} d x_{i, j}}{\operatorname{det} x^{n}}$ (where for $i<j$ the coordinates are in $\mathrm{E}$ and for $i=j$ the coordinates are in $\mathbf{F}$ ) and for any $x \in \mathrm{X}$ we take $\boldsymbol{\omega}_{\mathbf{G}^{x}}$ which matches $\boldsymbol{\omega}_{\mathbf{G}}$ and $\boldsymbol{\omega}_{\mathbf{X}}$. For any $\mathrm{M}$ we take the product form on $\mathbf{X}^{\mathbf{M}}=\mathbf{X} \cap \mathbf{M} \simeq \mathbf{X}_{n_{\mathbf{1}}} \times \cdots \times \mathbf{X}_{n_{t}}$. We take $\boldsymbol{\omega}_{\mathbf{M}}, y \in \mathrm{X}^{\mathrm{M}}$ to match $\boldsymbol{\omega}_{\mathbf{X}^{\mathbf{M}}}$ and $\boldsymbol{\omega}_{\mathbf{M}}$.

The factors $\boldsymbol{\lambda}_{\mathbf{v}}$ will be 1 for unipotent groups. In particular the Haar measure on $\mathrm{F}$ (resp. E) will be the self-dual Haar measure with respect to $\psi^{\prime}$ (resp. $\psi$ ). We take $\boldsymbol{\lambda}_{\mathbf{G}^{\prime}}=\prod_{i=1}^{n} \mathrm{~L}\left(i, 1_{\mathrm{F}^{*}}\right), \boldsymbol{\lambda}_{\mathbf{G}}=\prod_{i=1}^{n} \mathrm{~L}\left(i, 1_{\mathrm{E}^{*}}\right), \boldsymbol{\lambda}_{\mathbf{X}}=\prod_{i=1}^{n} \mathrm{~L}\left(i, \eta^{i+1}\right)$ and $\boldsymbol{\lambda}_{\mathbf{G}^{x}}=\prod_{i=1}^{n} \mathrm{~L}\left(i, \eta^{i}\right)$ where $\mathrm{L}(s, \chi)$ is the local L-factor of Tate. The isomorphism $\mathrm{X} \simeq \bigsqcup_{\xi \in \mathrm{X} / \mathrm{G}} \mathrm{G}^{\xi} \backslash \mathrm{G}$ is compatible with the measures. Similarly, we take $\boldsymbol{\lambda}_{\mathbf{M}}=\prod_{i=1}^{t} \boldsymbol{\lambda}_{\mathbf{G}_{\boldsymbol{n}_{\boldsymbol{i}}}}, \boldsymbol{\lambda}_{\mathbf{M}^{\prime}}=\prod_{i=1}^{t} \boldsymbol{\lambda}_{\mathbf{G}_{\boldsymbol{n}_{\boldsymbol{i}}}^{\prime}}$ and $\boldsymbol{\lambda}_{\mathbf{M}} \boldsymbol{y}=\prod_{i=1}^{t} \boldsymbol{\lambda}_{\mathbf{G}_{\boldsymbol{n}_{\boldsymbol{i}}}^{\boldsymbol{y}_{\boldsymbol{i}}}}$ for $y=\operatorname{diag}\left(y_{1}, \ldots, y_{t}\right) \in \mathrm{X}^{\mathrm{M}}$. If $\mathrm{F}$ is $p$-adic and $\psi^{\prime}$ has conductor $\mathcal{O}_{\mathrm{F}}$ then the measure on $\mathrm{G}^{\prime}$ gives $\operatorname{vol}\left(\mathrm{K}^{\prime}\right)=1$. If also $\mathrm{E} / \mathrm{F}$ is unramified then $\operatorname{vol}_{\mathrm{G}}(\mathrm{K})=\operatorname{vol}_{\mathrm{X}}(\mathrm{X} \cap \mathrm{K})=1$.

Given a closed subgroup $\mathrm{H}^{\prime} \subseteq \mathrm{H}$ we will consider the 'quotient measure' on $\mathrm{H}^{\prime} \backslash \mathrm{H}$ which is strictly speaking not necessarily a measure but rather a continuous functional on the space of continuous left $\left(\mathrm{H}^{\prime}, \frac{\delta_{\mathrm{H}^{\prime}}}{\delta_{\mathrm{H}}}\right)$-equivariant functions on $\mathrm{G}$ which are compactly supported modulo $\mathrm{H}^{\prime}$. Thus

$$
\int_{\mathrm{H}} f(h) d h=\int_{\mathrm{H}^{\prime} \backslash \mathrm{H}} \int_{\mathrm{H}^{\prime}} \frac{\delta_{\mathrm{H}}}{\delta_{\mathrm{H}^{\prime}}}\left(h^{\prime}\right) f\left(h^{\prime} x\right) d h^{\prime} d x
$$

for any compactly supported continuous function on $\mathrm{H}$. 
1.3. Representations and induced representations. - If $\mathrm{F}$ is $p$-adic then $\mathcal{R}(\mathrm{G})$ will denote the category of admissible finitely generated smooth representations (with respect to the discrete topology). In the Archimedean case, $\mathcal{R}(\mathrm{G})$ will denote the category of smooth Fréchet representations $(\pi, V)$ of moderate growth whose underlying $(\mathfrak{g}, \mathrm{K})$-module $\mathrm{V}_{\mathrm{K}}$ is admissible and finitely generated, with continuous intertwining operators as morphisms. $\left(\mathrm{V}_{\mathrm{K}}\right.$ determines $\mathrm{V}$ up to isomorphism - see [Wal92, Chapter 11 ]..$\left.^{2}\right)$ In both cases, any $\pi \in \mathcal{R}(\mathrm{G})$ is of finite length and can be realized as the smooth part of a Hilbert representation (say, on $\mathfrak{H}$ ) on which $\mathrm{K}$ (but not necessarily $\mathrm{G}$ ) acts unitarily. In the Archimedean case, let $\hat{\mathrm{K}}$ be the unitary dual of $\mathrm{K}$ and let $\mathfrak{H}=\hat{\bigoplus}_{\gamma \in \hat{\mathrm{K}}} \mathrm{V}^{\gamma}$ be the decomposition of $\mathfrak{H}$ into $\mathrm{K}$-types. Let $p_{\gamma}: \mathfrak{H} \rightarrow \mathrm{V}^{\gamma}, \gamma \in \hat{\mathrm{K}}$ be the corresponding orthogonal projections. We fix a norm on the Euclidean space spanned by the lattice of characters of a maximal torus of $\mathrm{K}$. For any $\gamma \in \hat{\mathrm{K}}$ let $\|\gamma\|$ be the norm of the highest weight of $\gamma$. Then $\mathrm{V}$ coincides with the space of $\mathrm{K}$-smooth vectors of $\mathfrak{H}$ and the topology of $\mathrm{V}$ is given by the norms

$$
\sum_{\gamma \in \hat{\mathrm{K}}}\left\|p_{\gamma}(v)\right\|_{\mathrm{V}^{\gamma}}(1+\|\gamma\|)^{n}, \quad n \in \mathbf{N} .
$$

(See e.g. [BK] for this and other basic facts about Harish-Chandra modules and their globalizations.)

Let $\left(\pi^{*}, V^{*}\right)$ be the representation on the topological dual of $V$ and let $\left(\pi^{\vee}, V^{\vee}\right) \in$ $\mathcal{R}(\mathrm{G})$ be the contragredient of $\pi$. As a representation space, $\mathrm{V}^{\vee}$ is the linear span of $\left\{\ell \circ \pi(f): \ell \in \mathrm{V}^{*}, f \in \mathcal{S}(\mathrm{G})\right\}$. In the $p$-adic case $\mathrm{V}^{\vee}$ is just the smooth part of $\mathrm{V}^{*}$. In the Archimedean case, suppose that $\pi$ is realized as the smooth part of a Hilbert representation of $\mathrm{G}$ on $\mathfrak{H}$. Then $\mathrm{V}^{\vee}$ is the smooth part of the dual Hilbert space of $\mathfrak{H}$ (isomorphic to the complex conjugate of $\mathfrak{H}$ ) with respect to the dual action. Thus, the Fréchet topology on $\mathrm{V}^{\vee}$ is given by the norms

$$
\ell \mapsto \sum_{\gamma \in \hat{\mathrm{K}}}\left\|\left.\ell\right|_{\mathrm{V}^{\gamma}}\right\|_{\left(\mathrm{V}^{\gamma}\right)^{*}}(1+\|\gamma\|)^{n}, \quad n \in \mathbf{N} .
$$

It will be useful to use different realizations of the contragredient representations. To that end, we say that the data $\mathcal{D}=(\pi, \hat{\pi},(\cdot, \cdot))$ (or simply $\pi, \hat{\pi}$ if $(\cdot, \cdot)$ is clear from the context) is a dual couple with respect to $\mathrm{G}$ if $\pi, \hat{\pi} \in \mathcal{R}(\mathrm{G})$ and $(\cdot, \cdot)$ is a pairing (i.e. a non-degenerate $\mathrm{G}$-invariant bilinear form) on $\pi \times \hat{\pi}$. This pairing gives rise to an equivalence of representations $\Lambda_{\mathcal{D}}: \pi^{\vee} \rightarrow \hat{\pi}$, and conversely, any such equivalence defines a dual couple.

By definition, an equivalence between the dual couples $\left(\pi_{1}, \hat{\pi}_{1},(\cdot, \cdot)_{1}\right)$ and $\left(\pi_{2}, \hat{\pi}_{2},(\cdot, \cdot)_{2}\right)$ is a pair $(\mathrm{A}, \hat{\mathrm{A}})$ of equivalences of representations $\mathrm{A}: \pi_{1} \rightarrow \pi_{2}$ and $\hat{\mathrm{A}}: \hat{\pi}_{1} \rightarrow \hat{\pi}_{2}$ such that $(\mathrm{A} v, \hat{\mathrm{A}} \hat{v})_{2}=(v, \hat{v})_{1}$ for any $v \in \pi_{1}, \hat{v} \in \hat{\pi}_{1}$. There is also an obvious notion of a direct sum of dual couples.

\footnotetext{
${ }^{2}$ Henceforth, equivalence of representations will always mean isomorphism as smooth representations.
} 
If $\pi \in \mathcal{R}(\mathrm{G})$ has a central character then we denote it by $\omega_{\pi}$. Given a character $\chi$ of $\mathrm{E}^{*}$, we denote by the same letter its pullback to $\mathrm{G}$ via det. For any $\pi \in \mathcal{R}(\mathrm{G})$ we set $\pi \cdot \chi=\pi \otimes(\chi \circ$ det $)$. This representation is realized on the same space as $\pi$ and $[\pi \cdot \chi](g)=\chi(g) \pi(g)$. In particular, we write $\pi[s]=\pi \cdot|\operatorname{det}|^{s}$. For any isomorphism $J$ between two groups $\mathrm{H}_{1}, \mathrm{H}_{2}$ and $(\pi, \mathrm{V}) \in \mathcal{R}\left(\mathrm{H}_{1}\right)$ we denote by $\left(\pi^{j}, \mathrm{~V}\right) \in \mathcal{R}\left(\mathrm{H}_{2}\right)$ the twist of $\pi$ by $J$, i.e., $\pi^{J}\left(g^{J}\right)=\pi(g), g \in \mathrm{H}_{1}$.

Let $(\sigma, \mathrm{V}) \in \mathcal{R}(\mathrm{M})$ and let $\mathrm{I}(\sigma)=\mathrm{I}_{\mathrm{M}}^{\mathrm{G}}(\sigma)$ be the space of smooth functions $\varphi$ on $\mathrm{G}$ with values in $\mathrm{V}$ such that

$$
\varphi(u m g)=\delta_{\mathrm{P}}(m)^{\frac{1}{2}} \sigma(m)[\varphi(g)], \quad m \in \mathrm{M}, u \in \mathrm{U}, g \in \mathrm{G} .
$$

We denote by $\varphi_{\lambda}, \lambda \in \mathfrak{a}_{\mathrm{M}, \mathbf{C}}^{*}$ the holomorphic section given by

$$
\varphi_{\lambda}(g)=e^{\langle\lambda, \mathrm{H}(g)\rangle} \varphi(g) .
$$

Let $\mathrm{I}(\sigma, \lambda)=\mathrm{I}_{\mathrm{M}}^{\mathrm{G}}(\sigma, \lambda)$ be the representation of $\mathrm{G}$ on the space $\mathrm{I}(\sigma)$ given by

$$
(\mathrm{I}(g, \sigma, \lambda) \varphi)_{\lambda}(x)=\varphi_{\lambda}(x g)
$$

for $x$ and $g$ in $\mathrm{G}$. This is the representation parabolically induced from $\sigma[\lambda]:=\sigma \cdot e^{\langle\lambda, \mathrm{H}(\cdot)\rangle}$ realized on the space $\mathrm{I}(\sigma)$. (Occasionally, we omit $\sigma$ from the notation if it is clear from the context.) Whenever $\sigma=\sigma_{1} \otimes \cdots \otimes \sigma_{t}, \sigma_{i} \in \mathcal{R}\left(\mathrm{G}_{n_{i}}\right)$ and $\lambda=\left(\lambda_{1}, \ldots, \lambda_{t}\right) \in \mathfrak{a}_{\mathrm{M}, \mathbf{C}}^{*}=\mathbf{G}^{t}$ we have

$$
\sigma[\lambda]=\sigma_{1}\left[\lambda_{1}\right] \otimes \cdots \otimes \sigma_{t}\left[\lambda_{t}\right]
$$

It will sometimes be convenient to denote the induced representation $\mathrm{I}(\sigma, 0)$ by $\sigma_{1} \times$ $\cdots \times \sigma_{t}$.

We use $\mathrm{I}_{\mathrm{M}}^{\mathrm{G}}$ as a functor. Thus, if $\sigma, \varrho \in \mathcal{R}(\mathrm{M})$ and $\mathrm{T}: \sigma \rightarrow \varrho$ is an intertwining operator then we write $\mathrm{I}_{\mathrm{M}}^{\mathrm{G}}(\mathrm{T}): \mathrm{I}_{\mathrm{M}}^{\mathrm{G}}(\sigma, \lambda) \rightarrow \mathrm{I}_{\mathrm{M}}^{\mathrm{G}}(\varrho, \lambda), \lambda \in \mathfrak{a}_{\mathrm{M}, \mathbf{C}}^{*}$ for the intertwining operator given by $\left[\mathrm{I}_{\mathrm{M}}^{\mathrm{G}}(\mathrm{T}) \varphi\right](g)=\mathrm{T}[\varphi(g)], g \in \mathrm{G}, \varphi \in \mathrm{I}(\sigma)$.

More generally, if $v \in \mathcal{R}(\mathrm{L})$ and $\mu \in \mathfrak{a}_{\mathrm{L}, \mathbf{G}}^{*}$, we denote by $\mathrm{I}_{\mathrm{L}}^{\mathrm{M}}(\nu, \mu) \in \mathcal{R}(\mathrm{M})$ the parabolic induction from $v[\mu]$ realized on the space $\mathrm{I}_{\mathrm{L}}^{\mathrm{M}}(\nu)$. The associated action of $m \in \mathrm{M}$ on $\mathrm{I}_{\mathrm{L}}^{\mathrm{M}}(\nu)$ is denoted by $\mathrm{I}_{\mathrm{L}}^{\mathrm{M}}(m, \nu, \mu)$ and $\varphi_{\mu}=e^{\langle\mu, \mathrm{H}(\cdot)\rangle} \varphi$ is the holomorphic section associated with $\varphi \in \mathrm{I}_{\mathrm{L}}^{\mathrm{M}}(\nu)$. For $\varphi \in \mathrm{I}_{\mathrm{L}}^{\mathrm{G}}(\nu)$ we write $\varphi_{\mathrm{M}}=\left.\delta_{\mathrm{P}}^{-\frac{1}{2}} \varphi\right|_{\mathrm{M}} \in \mathrm{I}_{\mathrm{L}}^{\mathrm{M}}(v)$. With this notation, transitivity of induction can be expressed as follows. Suppose that $\sigma=\mathrm{I}_{\mathrm{L}}^{\mathrm{M}}(\nu, \mu)$. For $\varphi \in \mathrm{I}_{\mathrm{L}}^{\mathrm{G}}(\nu)$ and $\xi \in \mathrm{I}_{\mathrm{M}}^{\mathrm{G}}(\sigma)$ we write $\varphi \stackrel{\mu}{\leftrightarrows} \xi$ if we have

$$
\varphi_{\mu}(g)=\xi(g)(e), \quad g \in \mathrm{G},
$$

or equivalently,

$$
(\xi(g))_{\mu}(m)=\delta_{\mathrm{P}}^{-\frac{1}{2}}(m) \varphi_{\mu}(m g)=\left([\mathrm{I}(g, \nu, \mu) \varphi]_{\mathrm{M}}\right)_{\mu}(m) .
$$


It will sometimes be convenient to write

$$
\Gamma_{\mathrm{L}, \mathrm{M}}(\nu, \mu)=\Gamma_{\mathrm{L}, \mathrm{M}}^{\mathrm{G}}(\nu, \mu): \mathrm{I}_{\mathrm{L}}^{\mathrm{G}}(\nu, \mu+\lambda) \rightarrow \mathrm{I}_{\mathrm{M}}^{\mathrm{G}}(\sigma, \lambda)
$$

for the equivalence (for any $\lambda \in \mathfrak{a}_{\mathrm{M}, \mathbf{C}}^{*}$ ) given by

$$
\Gamma_{\mathrm{L}, \mathrm{M}}^{\mathrm{G}}(\nu, \mu) \varphi(g)=[\mathrm{I}(g, v, \mu) \varphi]_{\mathrm{M}} .
$$

Thus,

$$
\varphi \stackrel{\mu}{\leftrightarrows} \Gamma_{\mathrm{L}, \mathrm{M}}^{\mathrm{G}}(\nu, \mu) \varphi
$$

We will also denote the (normalized) Jacquet functor from smooth representations of $\mathrm{M}$ to smooth representations of $\mathrm{L}$ by $r_{\mathrm{L}, \mathrm{M}}$. The Jacquet functor from $\mathrm{G}$ to $\mathrm{M}$ will simply be denoted by $r_{\mathrm{M}}$.

1.4. Jacquet integral, intertwining operators and gamma factors. - Let $\psi_{0}^{\prime}$ be the character of $\mathrm{U}_{0}^{\prime}$ defined by

$$
\psi_{0}^{\prime}(u)=\psi^{\prime}\left(u_{1,2}+\cdots+u_{n-1, n}\right)
$$

and let $\psi_{0}$ be the character of $\mathrm{U}_{0}$ defined similarly with respect to $\psi$.

Henceforth we denote by $\Pi$ the set of equivalence classes of irreducible representations of $\mathrm{G}$. We have

$$
\Pi_{\text {cusp }} \subseteq \Pi_{\text {sqr }} \subseteq \Pi_{\text {gen }} \subseteq \Pi
$$

where the notation stands for the subsets of (not necessarily unitary) supercuspidal (in the $p$-adic case), essentially square-integrable and generic representations, respectively. We also write $\Pi_{\text {usqr }} \subseteq \Pi_{\text {temp }} \subseteq \Pi_{\text {gen }}$ for the subsets of (unitary) square-integrable and tempered representations respectively. As usual, analogous notation for $\mathrm{G}^{\prime}$ will be appended with a prime. We write $\Pi^{\tau}$ for the irreducible representations of $\mathrm{G}$ which are equivalent to their Galois twist. Similarly for $\Pi_{\text {cusp }}^{\tau}$, etc.

For $\pi \in \Pi_{\text {gen }}$ denote by $\mathcal{W}(\pi)=\mathcal{W}^{\psi}(\pi)$ its Whittaker model with respect to $\left(\mathrm{U}_{0}, \psi_{0}\right)$. For every $g \in \mathrm{G}$ denote by $\delta_{g}^{\pi}$ the evaluation at $g$ viewed as a linear form on $\mathcal{W}(\pi)$ and let

$$
(\mathcal{W}(g, \pi) \mathrm{W})(h)=\mathrm{W}(h g), \quad g, h \in \mathrm{G}, \mathrm{W} \in \mathcal{W}(\pi)
$$

Let $\tilde{\theta}=\tilde{\theta}_{n}$ be the involution of $\mathrm{G}$ given by $g \mapsto w_{0}{ }^{t} g^{-1} w_{0}$. It preserves $\mathrm{U}_{0}$ and we have $\psi_{0}\left(u^{\tilde{\theta}}\right)=\psi_{0}(u)^{-1}$ for all $u \in \mathrm{U}_{0}$. Given $\pi \in \Pi_{\text {gen }}$ we have an equivalence

$$
\mathfrak{y}_{\pi}: \mathcal{W}^{\psi}(\pi)^{\tilde{\theta}} \rightarrow \mathcal{W}^{\psi^{-1}}\left(\pi^{\vee}\right)
$$

given by $\mathfrak{y}_{\pi}(\mathrm{W})(g)=\mathrm{W}\left(g^{\tilde{\theta}}\right), g \in \mathrm{G}$. 
For $\mathbf{P}=\mathbf{M U}$ as above we have $\tilde{\theta}(\mathbf{U})=\overleftarrow{\mathbf{U}}, w_{\mathrm{M}}^{\tilde{\theta}}=w_{\overleftarrow{\mathrm{M}}}=w_{\mathrm{M}}^{-1}, \mathrm{H}\left(g^{\tilde{\theta}}\right)=-w_{0} \mathrm{H}(g)$ $g \in \mathrm{G}$, and $\operatorname{diag}\left(m_{1}, \ldots, m_{t}\right)^{\tilde{\theta}}=\operatorname{diag}\left(m_{t}^{\tilde{\theta}_{n_{s}}}, \ldots, m_{1}^{\tilde{\theta}_{n_{1}}}\right), m_{i} \in \mathrm{G}_{n_{i}}$.

Let $\sigma=\sigma_{1} \otimes \cdots \otimes \sigma_{t} \in \Pi_{\text {gen }}^{\mathrm{M}}$. We identify $\mathcal{W}(\sigma)$ with $\mathcal{W}\left(\sigma_{1}\right) \otimes \cdots \otimes \mathcal{W}\left(\sigma_{t}\right)$. For any $\lambda \in \mathfrak{a}_{\mathrm{M}, \mathbf{C}}^{*}$ and $\varphi \in \mathrm{I}(\mathcal{W}(\sigma))$ we denote by $\mathbf{W}(\varphi, \sigma, \lambda)$ the holomorphic continuation of the Jacquet integral (see [Jac67])

$$
\mathbf{W}(g: \varphi, \sigma, \lambda)=\mathbf{W}_{\mathrm{M}}(g: \varphi, \sigma, \lambda)=\int_{\overleftarrow{\mathrm{U}}} \delta_{e}^{\sigma}\left(\varphi_{\lambda}\left(w_{\mathrm{M}}^{-1} u g\right)\right) \psi_{0}^{-1}(u) d u
$$

We view $\mathbf{W}(\sigma, \lambda)$ as an intertwining operator from $\mathrm{I}(\mathcal{W}(\sigma), \lambda)$ to the space of Whittaker functions on $\mathrm{G}$. We also write

$$
\mathbf{W}_{e}(\varphi: \sigma, \lambda)=\mathbf{W}(e: \varphi, \sigma, \lambda)
$$

so that $\mathbf{W}_{e}(\sigma, \lambda)$ is a Whittaker functional on $\mathrm{I}(\mathcal{W}(\sigma), \lambda)$. If $\mathrm{I}(\sigma, \lambda)$ is irreducible then $\mathrm{I}(\sigma, \lambda) \in \Pi_{\text {gen }}$ and

$$
\mathbf{W}(\sigma, \lambda): \mathrm{I}(\mathcal{W}(\sigma), \lambda) \rightarrow \mathcal{W}(\mathrm{I}(\sigma, \lambda))
$$

is an equivalence of representations.

Let $\overleftarrow{\sigma}=\sigma_{t} \otimes \cdots \otimes \sigma_{1} \in \Pi_{\text {gen }}^{\overleftarrow{M}}$ and define $\mathfrak{y}_{\sigma}: \mathcal{W}^{\psi}(\overleftarrow{\sigma})^{\tilde{\theta}} \rightarrow \mathcal{W}^{\psi^{-1}}\left(\sigma^{\vee}\right)$ by $\mathfrak{y}_{\sigma}(\mathrm{W})(g)=\mathrm{W}\left(g^{\tilde{\theta}}\right), g \in \mathrm{M}$. For any $\lambda=\left(\lambda_{1}, \ldots, \lambda_{t}\right) \in \mathfrak{a}_{\mathrm{M}, \mathbf{C}}^{*}$ we write $\overleftarrow{\lambda}=w_{\mathrm{M}} \lambda=$ $\left(\lambda_{t}, \ldots, \lambda_{1}\right) \in \mathfrak{a}_{\overleftarrow{M}, \mathbf{C}}^{*}$. Define

$$
\Theta(\sigma, \lambda):\left[\mathrm{I}_{\overleftarrow{\mathrm{M}}}\left(\mathcal{W}^{\psi}(\overleftarrow{\sigma}), \overleftarrow{\lambda}\right)\right]^{\tilde{\theta}} \rightarrow \mathrm{I}_{\mathrm{M}}\left(\mathcal{W}^{\psi^{-1}}\left(\sigma^{\vee}\right),-\lambda\right)
$$

by $\Theta(\varphi)\left(g^{\tilde{\theta}}\right)=\mathfrak{y}_{\sigma}(\varphi(g)), g \in \mathrm{G}$. Then $\Theta(\sigma, \lambda)$ is an intertwining operator and if $\pi=$ $\mathrm{I}(\sigma, \lambda)$ is irreducible then we have

$$
\mathfrak{y}_{\pi} \circ \mathbf{W}_{\overleftarrow{M}}(\overleftarrow{\sigma}, \overleftarrow{\lambda})=\mathbf{W}_{M}^{\psi^{-1}}\left(\sigma^{\vee},-\lambda\right) \circ \Theta
$$

Indeed, by a change of variable in the integral defining $\mathbf{W}_{e}$ we have

$$
\begin{aligned}
\mathbf{W}_{e}^{\psi}(\varphi: \overleftarrow{\sigma}, \overleftarrow{\lambda}) & =\int_{\mathrm{U}} \delta_{e}^{\overleftarrow{\sigma}}\left(\varphi_{\overleftarrow{\lambda}}\left(w_{\mathrm{M}} u\right)\right) \psi_{0}(u)^{-1} d u \\
& =\int_{\overleftarrow{\mathrm{U}}} \delta_{e}^{\overleftarrow{\sigma}}\left(\varphi_{\overleftarrow{\lambda}}\left(\left(w_{\overleftarrow{\mathrm{M}}} u\right)^{\tilde{\theta}}\right)\right) \psi_{0}(u) d u \\
& =\int_{\overleftarrow{\mathrm{U}}} \delta_{e}^{\sigma^{\vee}}\left((\Theta \varphi)_{-\lambda}\left(w_{\overleftarrow{\mathrm{M}}} u\right)\right) \psi_{0}(u) d u \\
& =\mathbf{W}_{e}^{\psi^{-1}}\left(\Theta \varphi: \sigma^{\vee},-\lambda\right)
\end{aligned}
$$

for any $\varphi \in \mathrm{I}_{\overleftarrow{\mathrm{M}}}\left(\mathcal{W}^{\psi}(\overleftarrow{\sigma})\right)$ 
we obtain

If $v \in \Pi_{\text {gen }}^{\mathrm{L}}, \mu \in \mathfrak{a}_{\mathrm{L}, \mathbf{G}}^{*}$ and $\sigma=\mathrm{I}_{\mathrm{L}}^{\mathrm{M}}(\mathcal{W}(v), \mu) \in \Pi_{\text {gen }}^{\mathrm{M}}$ then using integration in stages

$$
\mathbf{W}_{\mathrm{L}}(\nu, \mu+\lambda)=\mathbf{W}_{\mathrm{M}}(\sigma, \lambda) \circ \mathrm{I}_{\mathrm{M}}^{\mathrm{G}}\left(\mathbf{W}_{\mathrm{L}}^{\mathrm{M}}(\nu, \mu)\right) \circ \Gamma_{\mathrm{L}, \mathrm{M}}(\mathcal{W}(v), \mu) .
$$

For any $w \in \mathrm{W}(\mathrm{M})$ denote by $w \sigma$ the representation of $\mathrm{M}_{w \kappa}$ on $\mathrm{V}$ defined by $w \sigma\left(w m w^{-1}\right)=\sigma(m)$. There is an intertwining operator $\mathrm{M}(w, \sigma, \lambda): \mathrm{I}_{\mathrm{M}}(\sigma, \lambda) \rightarrow$ $\mathrm{I}_{\mathrm{M}_{w \kappa}}(w \sigma, w \lambda)$ defined as the meromorphic continuation of the integral

$$
(\mathrm{M}(w, \sigma, \lambda) \varphi)_{w \lambda}(g)=\int_{\left(\mathrm{U}_{w \kappa} \cap w \mathrm{U} w^{-1}\right) \backslash \mathrm{U}_{w \kappa}} \varphi_{\lambda}\left(w^{-1} u g\right) d u .
$$

If $\sigma \in \Pi_{\text {gen }}^{\mathrm{M}}$ then we can identify $w \mathcal{W}(\sigma)$ with $\mathcal{W}(w \sigma)$ by $\mathrm{W} \rightarrow \mathrm{W}\left(w \cdot w^{-1}\right)$. By a slight abuse of notation, we write $\mathrm{M}(w, \mathcal{W}(\sigma), \lambda)$ for the intertwining operator $\mathrm{I}_{\mathrm{M}}(\mathcal{W}(\sigma), \lambda) \rightarrow \mathrm{I}_{\mathrm{M}_{w \kappa}}(\mathcal{W}(w \sigma), w \lambda)$ obtained using this identification. For future record, we note that for $\varphi \in \mathrm{I}_{\mathrm{M}}(\mathcal{W}(\sigma), \lambda)$ we have

$$
\begin{gathered}
{\left[\Theta(\sigma, \lambda) \circ \mathrm{M}\left(w_{\mathrm{M}}, \mathcal{W}(\sigma), \lambda\right) \varphi\right]_{-\lambda}(g)=\int_{\overleftarrow{\mathrm{U}}} \mathfrak{y}_{\sigma}\left(\varphi_{\lambda}\left(w_{\mathrm{M}}^{-1} u g^{\tilde{\theta}}\right)\right) d u} \\
=\int_{\mathrm{U}} \mathfrak{y} \overleftarrow{\sigma}\left(\varphi_{\lambda}\left(w_{\mathrm{M}}^{-1} u^{\tilde{\theta}} g^{\tilde{\theta}}\right)\right) d u=\int_{\mathrm{U}} \mathfrak{y} \overleftarrow{\sigma}\left(\varphi_{\lambda}\left(w_{0}^{\mathrm{M} t} u^{-1} w_{0} g^{\tilde{\theta}}\right)\right) d u
\end{gathered}
$$

where for the first equality we identified $\mathfrak{y}_{\sigma}$ on $\mathcal{W}(\overleftarrow{\sigma})=\mathcal{W}\left(w_{\mathrm{M}} \sigma\right)$ as $\mathfrak{y}_{\overleftarrow{\sigma}}$ on $w_{\mathrm{M}} \mathcal{W}(\sigma)$

Denote by $\mathcal{R}_{\mathrm{pi}}(\mathrm{G})$ the representations in $\mathcal{R}(\mathrm{G})$ which are parabolically induced from an irreducible representation on a Levi subgroup of $\mathrm{G}$. Let $\pi_{i} \in \mathcal{R}_{\mathrm{pi}}\left(\mathrm{G}_{n_{i}}\right), i=1,2$ and recall the local Rankin-Selberg factors

$$
\gamma\left(s, \pi_{1} \times \pi_{2} ; \psi\right)=\frac{\varepsilon\left(s, \pi_{1} \times \pi_{2} ; \psi\right) \mathrm{L}\left(1-s, \pi_{1}^{\vee} \times \pi_{2}^{\vee}\right)}{\mathrm{L}\left(s, \pi_{1} \times \pi_{2}\right)}
$$

defined by Jacquet, Piatetski-Shapiro and Shalika [JPSS83] in the non-Archimedean case. In the Archimedean case they are defined via Langlands parameterization in terms of representations of the Weil group (cf. [Sha85]). If $\pi_{1}=\sigma_{1} \times \cdots \times \sigma_{t}$ then we have

$$
\begin{aligned}
& \mathrm{L}\left(s, \pi_{1} \times \pi_{2}\right)=\prod_{i=1}^{t} \mathrm{~L}\left(s, \sigma_{i} \times \pi_{2}\right), \\
& \varepsilon\left(s, \pi_{1} \times \pi_{2} ; \psi\right)=\prod_{i=1}^{t} \varepsilon\left(s, \sigma_{i} \times \pi_{2} ; \psi\right)
\end{aligned}
$$

and therefore also

$$
\gamma\left(s, \pi_{1} \times \pi_{2} ; \psi\right)=\prod_{i=1}^{t} \gamma\left(s, \sigma_{i} \times \pi_{2} ; \psi\right) .
$$


We recall Shahidi's normalization for the intertwining operators. Let $\sigma=\sigma_{1} \otimes$ $\cdots \otimes \sigma_{t} \in \mathcal{R}_{\mathrm{pi}}(\mathrm{M}), w \in \mathrm{W}(\mathrm{M})$ (considered as a permutation of $[1, t]$ as before) and $\lambda=$ $\left(\lambda_{1}, \ldots, \lambda_{t}\right) \in \mathbf{G}^{t}=\mathfrak{a}_{\mathrm{M}, \mathbf{G}}^{*}$. Let

$$
\mathrm{C}_{\mathrm{M}}(w: \sigma, \lambda ; \psi)=\prod_{\substack{1 \leq i<j \leq t \\ w(i)>w(j)}} \omega_{\sigma_{j}}(-1)^{n_{i}} \gamma\left(\lambda_{i}-\lambda_{j}, \sigma_{i} \times \sigma_{j}^{\vee} ; \psi\right)
$$

and

$$
\mathfrak{c s}(\sigma, \lambda)=\prod_{i<j} \mathrm{~L}\left(\lambda_{i}-\lambda_{j}+1, \sigma_{i} \times \sigma_{j}^{\vee}\right)
$$

Define the normalized intertwining operators

$$
\mathrm{N}(w, \sigma, \lambda)=\mathrm{C}_{\mathrm{M}}(w: \sigma, \lambda ; \psi) \mathrm{M}(w, \sigma, \lambda): \mathrm{I}_{\mathrm{M}}(\sigma, \lambda) \rightarrow \mathrm{I}_{w \mathrm{M} w^{-1}}(w \sigma, w \lambda)
$$

and (in the case $\sigma \in \Pi_{\text {gen }}^{\mathrm{M}}$ )

$$
\begin{aligned}
\mathrm{N}(w, \mathcal{W}(\sigma), \lambda)= & \mathrm{C}_{\mathrm{M}}(w: \sigma, \lambda ; \psi) \mathrm{M}(w, \mathcal{W}(\sigma), \lambda): \mathrm{I}_{\mathrm{M}}(\mathcal{W}(\sigma), \lambda) \\
& \rightarrow \mathrm{I}_{w \mathrm{M} w^{-1}}(\mathcal{W}(w \sigma), w \lambda)
\end{aligned}
$$

Suppose that $\sigma \in \Pi_{\text {gen }}^{\mathrm{M}}$. It follows from the results of Shahidi (cf. [Sha90]) that we have the identity of meromorphic functions in $\lambda \in \mathfrak{a}_{\mathrm{M}, \mathbf{C}}^{*}$

$$
\mathbf{W}(w \sigma, w \lambda) \circ \mathrm{N}(w, \mathcal{W}(\sigma), \lambda)=\mathbf{W}(\sigma, \lambda),
$$

and in particular

$$
\mathbf{W}_{e}(w \sigma, w \lambda) \circ \mathrm{N}(w, \mathcal{W}(\sigma), \lambda)=\mathbf{W}_{e}(\sigma, \lambda)
$$

We also have

$$
\mathrm{N}\left(w_{2} w_{1}, \sigma, \lambda\right)=\mathrm{N}\left(w_{2}, w_{1} \sigma, w_{1} \lambda\right) \circ \mathrm{N}\left(w_{1}, \sigma, \lambda\right)
$$

for any $w_{1} \in \mathrm{W}(\mathrm{M})$ and $w_{2} \in \mathrm{W}\left(w_{1} \mathrm{M} w_{1}^{-1}\right)$. In particular, for any $w \in \mathrm{W}(\mathrm{M})$

$$
\mathrm{N}(w, \sigma, \lambda)^{-1}=\mathrm{N}\left(w^{-1}, w \sigma, w \lambda\right) .
$$

${ }^{3}$ The representatives for $\mathrm{W}$ specified in [Sha90] are different from ours. We refer the reader to the earlier paper [Sha84] where the case of $\mathrm{GL}(n)$ is considered. 
1.5. Irreducibility of parabolic induction. - We recall the following basic and wellknown properties of $\gamma$-factors combining the results of [JPSS83, §8] and [MW89, §I.6]. ${ }^{4}$

Lemma 1.1. - Suppose that $\delta=\delta_{1} \otimes \delta_{2} \in \Pi_{\mathrm{usqr}}^{\mathrm{M}}$ (M maximal). Then

(1) $\mathrm{L}\left(s, \delta_{1} \times \delta_{2}^{\vee}\right)$ and $\gamma\left(s, \delta_{1} \times \delta_{2}^{\vee} ; \psi\right)^{-1}$ are holomorphic for $\operatorname{Re} s>0$. Consequently, $\gamma\left(s, \delta_{1} \times \delta_{2}^{\vee} ; \psi\right)$ is holomorphic for $\operatorname{Re} s<1$.

(2) $\mathrm{L}\left(s, \delta_{1} \times \delta_{2}^{\vee}\right)$, or equivalently, $\gamma\left(s, \delta_{1} \times \delta_{2}^{\vee} ; \psi\right)^{-1}$, has a pole, necessarily simple, at $s=0$ if and only if $\delta_{2}=\delta_{1}$.

(3) $\mathrm{M}\left(w_{\mathrm{M}}, \delta, \lambda\right)$ and $\mathrm{N}\left(w_{\mathrm{M}}, \delta,-\lambda\right)$ are holomorphic for $\operatorname{Re} \lambda_{1}>\operatorname{Re} \lambda_{2}$.

(4) Suppose that $\operatorname{Re} \lambda_{1}>\operatorname{Re} \lambda_{2}$. Then $\pi:=\mathrm{I}(\delta, \lambda)$ is reducible if and only if $\gamma\left(\lambda_{1}-\right.$ $\left.\lambda_{2}, \delta_{1} \times \delta_{2}^{\vee} ; \psi\right)=\infty$, i.e., if and only if $\mathrm{L}\left(1-\lambda_{1}+\lambda_{2}, \delta_{1}^{\vee} \times \delta_{2}\right)=\infty$. In this case, at least in the p-adic and complex cases, ${ }^{5} \pi$ has length two and admits a unique irreducible subrepresentation $\sigma$, which is generic. We have $\sigma=\operatorname{Ker} M\left(w_{\mathrm{M}}, \delta, \lambda\right)=$ $\operatorname{Im} \mathrm{N}\left(w_{\mathrm{M}}, \delta, \lambda\right)^{-1}=\operatorname{Im} \mathrm{N}\left(w_{\mathrm{M}}^{-1}, w_{\mathrm{M}} \delta, w_{\mathrm{M}} \lambda\right)$.

In particular, in the p-adic case, the following conditions are equivalent for $\sigma_{1}, \sigma_{2} \in \Pi_{\text {cusp }}$ (not necessarily unitary)

(1) $\gamma\left(0, \sigma_{1} \times \sigma_{2}^{\vee} ; \psi\right)=0$.

(2) $\gamma\left(1, \sigma_{1} \times \sigma_{2}^{\vee} ; \psi\right)=\infty$

(3) $\sigma_{2}=\sigma_{1}$.

By the Langlands classification, for any $\pi \in \Pi$ there exists a unique triplet $(\mathrm{M}, \sigma, \lambda)$ (Langlands data) where $\sigma \in \Pi_{\text {temp }}^{\mathrm{M}}$ and $\lambda \in\left(\mathfrak{a}_{\mathrm{M}}^{*}\right)_{+}$such that $\pi$ is the unique irreducible quotient of $\mathrm{I}(\sigma, \lambda)$. We write $\pi=\mathrm{LQ}(\sigma, \lambda)$ in this case. Recall that $\mathrm{C}_{\mathrm{M}}\left(w_{\mathrm{M}}\right.$ : $\sigma, \cdot ; \psi)^{-1}$ is holomorphic at $\lambda$ and

$$
\pi \in \Pi_{\text {gen }} \Longleftrightarrow \mathrm{C}_{\mathrm{M}}\left(w_{\mathrm{M}}: \sigma, \lambda ; \psi\right)^{-1} \neq 0 \quad \Longleftrightarrow \mathrm{I}(\sigma, \lambda) \text { is irreducible. }
$$

See [CS98, §5] for a more general statement.

Alternatively, we can write any $\pi \in \Pi_{\text {gen }}$ as $\mathrm{I}(\delta, \lambda)$ where $\delta \in \Pi_{\text {usqr }}^{\mathrm{M}}$ and $\lambda \in \overline{\left(\mathfrak{a}_{\mathrm{M}}^{*}\right)_{+}}$, i.e., $\lambda_{1} \geq \cdots \geq \lambda_{t}$.

Let $\pi \in \Pi_{\text {gen }}$. Write $\pi=\sigma_{1} \times \cdots \times \sigma_{t}$ where $\sigma_{1} \otimes \cdots \otimes \sigma_{t} \in \Pi_{\text {sqr }}^{\mathrm{M}}$. Then $\mathrm{L}(s, \pi \times$ $\left.\pi^{\vee}\right)=\prod_{1 \leq i, j \leq t} \mathrm{~L}\left(s, \sigma_{i} \times \sigma_{j}^{\vee}\right)$. The latter is holomorphic at $s=1$ by Lemma 1.1 and the irreducibility of $\pi$. It follows that

$$
\mathrm{L}\left(s, \pi \times \pi^{\vee}\right) \quad \text { is holomorphic at } s=1 \text { for any } \pi \in \Pi_{\text {gen }} .
$$

We write $\Pi_{\text {unr }}$ for the class of unramified representations in $\Pi$, i.e., those which admit a non-zero spherical (i.e., K-fixed) vector, which is necessarily unique up to a scalar.

\footnotetext{
${ }^{4}$ Note the following typo in [JPSS83, Proposition 8.1]: $\mathrm{L}(s, \pi \times \sigma)$ should be replaced by $\mathrm{L}\left(s, \pi \times \sigma^{\vee}\right)$. Also note that our convention for the normalized intertwining operator $\mathrm{N}$ is different from [MW89].

${ }^{5}$ Probably also in the real case, but we haven't been able to find a reference for that.
} 
Note that $\pi \in \Pi_{\text {unr }}$ if and only if $\pi^{\vee} \in \Pi_{\text {unr }}$. For any bi-K-invariant $f \in \mathcal{S}(\mathrm{G})$ and $\pi \in$ $\Pi_{\text {unr }}$, we write $\hat{f}(\pi)$ for the scalar by which $\pi(f)$ acts on the spherical vector. This is called the Harish-Chandra transform. More explicitly, if $\pi$ is the unramified subquotient of $\mathrm{I}\left(1_{\mathrm{M}_{0}}, \lambda\right)$ then

$$
\hat{f}(\pi)=\int_{\mathrm{G}} f(g) e^{\left\langle\rho_{0}+\lambda, \mathrm{H}(g)\right\rangle} d g .
$$

Suppose now that $\mathrm{F}$ is $p$-adic. For any $\pi \in \Pi$ there exists $\mathrm{M}$ and $\sigma=\sigma_{1} \otimes \cdots \otimes$ $\sigma_{t} \in \Pi_{\text {cusp }}^{\mathrm{M}}$ such that $\pi$ is a subquotient of $\mathrm{I}(\sigma)$. The multiset $\operatorname{supp}_{c}(\pi)=\left\{\sigma_{1}, \ldots, \sigma_{t}\right\}$ is determined by $\pi$ and is called the supercuspidal support of $\pi$. We say that $\pi$ is pure (of type $\sigma)$ if there exists a divisor $m$ of $n$ and a representation $\sigma \in \Pi_{\text {cusp }}^{\mathrm{G}_{m}}$ such that $\operatorname{supp}_{c}(\pi) \subseteq$ $\sigma[\mathbf{Z}]:=\{\sigma[k]: k \in \mathbf{Z}\}$. Note that if $\pi$ is pure of type $\sigma$ then $\pi \in \Pi^{\tau}$ if and only if $\sigma \in \Pi^{\tau}$. This follows for instance from the Zelevinsky classification. We say that $\pi_{1}, \pi_{2} \in \Pi$ are disjoint if the $\operatorname{sets} \operatorname{supp}_{c}\left(\pi_{2}\right)$ and $\sigma[\mathbf{Z}]$ are disjoint for every $\sigma \in \operatorname{supp}_{c}\left(\pi_{1}\right)$. If $\pi_{1}, \pi_{2}$ are disjoint then $\pi_{1} \times \pi_{2}$ is irreducible. Any $\pi \in \Pi$ can be written as $\delta_{1} \times \cdots \times \delta_{t}$ where the $\delta_{i}$ 's are pure and pairwise disjoint. The $\delta_{i}$ 's are unique up to permutation and are called the pure components of $\pi$.

For any $\sigma \in \Pi_{\text {cusp }}$ and $a, b \in \mathbf{Z}$ with $a \leq b$ the induced representation $\sigma[a] \times \cdots \times$ $\sigma[b]$ admits a unique irreducible quotient $\Delta_{\sigma}([a, b])$. We have $\Delta_{\sigma}([a, b]) \in \Pi_{\text {sqr }}$. Conversely, any $\pi \in \Pi_{\text {sqr }}$ is of the form above. Also, $\Delta_{\sigma_{1}}\left[a_{1}, b_{1}\right]=\Delta_{\sigma_{2}}\left[a_{2}, b_{2}\right]$ if and only if $\sigma_{1}\left[a_{1}\right]=\sigma_{2}\left[a_{2}\right]$ and $\sigma_{1}\left[b_{1}\right]=\sigma_{2}\left[b_{2}\right]$. We have $\Delta_{\sigma}([a, b])^{\tau}=\Delta_{\sigma^{\tau}}([a, b])$ and therefore $\Delta_{\sigma}([a, b]) \in \Pi_{\text {sqr }}^{\tau}$ if and only if $\sigma \in \Pi_{\text {cusp }}^{\tau}$.

Let $\sigma \in \Pi_{\text {cusp }}^{\mathrm{G}_{d}}$. The Jacquet module of $\Delta_{\sigma}([a, b])$ was described by Zelevinsky as follows [Zel80, §9.5]. Let $\mathrm{M}=\mathrm{M}_{\kappa}$ where $\kappa=\left(n_{1}, \ldots, n_{t}\right)$. If $d$ does not divide $n_{i}$ for some $i$ then $r_{\mathrm{M}}\left(\Delta_{\sigma}([a, b])\right)=0$. Otherwise,

$$
r_{\mathrm{M}}\left(\Delta_{\sigma}([a, b])\right)=\bigotimes_{i=1}^{t} \Delta_{\sigma}\left(\left[a_{i}, b_{i}\right]\right)
$$

where $b_{1}=b, d\left(b_{i}-a_{i}+1\right)=n_{i}, i=1, \ldots, t$ and $b_{i+1}=a_{i}-1, i=1, \ldots, t-1$.

Let $\delta_{i} \in \Pi_{\mathrm{sqr}}^{\mathrm{G}_{n_{i}}}, i=1,2$. We write $\delta_{1} \unlhd \delta_{2}$ if for some $\sigma \in \Pi_{\text {cusp }}$ and integers $a_{1} \leq$ $a_{2} \leq b_{1} \leq b_{2}$ we have $\delta_{1}=\Delta_{\sigma}\left(\left[a_{1}, b_{1}\right]\right)$ and $\delta_{2}=\Delta_{\sigma}\left(\left[a_{2}, b_{2}\right]\right)$. If moreover $\delta_{1} \neq \delta_{2}$ then we write $\delta_{1} \triangleleft \delta_{2}$. It follows from the above description of the Jacquet module that $\delta_{1} \unlhd \delta_{2}$ if and only if there exist Levi subgroups $\mathrm{M}_{i}$ of $\mathrm{G}_{n_{i}}, i=1,2$ (not necessarily proper) and $\tilde{\delta} \in \Pi_{\text {sqr }}$ such that $r_{\mathrm{M}_{1}}\left(\delta_{1}\right)=\tilde{\delta} \otimes \cdots \otimes \cdots$ and $r_{\mathrm{M}_{2}}\left(\delta_{2}\right)=\cdots \otimes \cdots \otimes \tilde{\delta}$. Note that when $\delta_{1}=\delta_{2}$, this condition is only possible for $\mathrm{M}_{1}=\mathrm{M}_{2}=\mathrm{G}_{n_{1}}=\mathrm{G}_{n_{2}}$.

\section{Bessel distributions}

2.1. General setup and basic properties. - We first recall and slightly extend the notion of Bessel distributions (cf. [JLR04, §4.1]). Let $\pi \in \mathcal{R}(\mathrm{G})$. The map $\ell \mapsto(f \mapsto \ell \circ \pi(f))$ 
defines an equivalence of representations

$$
\pi^{*} \simeq \operatorname{Hom}_{\mathrm{G}}\left(\mathcal{S}(\mathrm{G})^{r}, \pi^{\vee}\right)
$$

where $\mathcal{S}(\mathrm{G})^{r}$ stands for $\mathcal{S}(\mathrm{G})$ with the right $\mathrm{G}$-action and $\mathrm{G}$ acts on the right-hand side through its left action on $\mathcal{S}(\mathrm{G})$.

Let $\mathcal{D}=(\pi, \hat{\pi},(\cdot, \cdot))$ be a dual couple (see Section 1.3) and recall the ensuing equivalence $\Lambda_{\mathcal{D}}: \pi^{\vee} \rightarrow \hat{\pi}$. For $\ell \in \pi^{*}$ and $\hat{\ell} \in \hat{\pi}^{*}$ define

$$
\mathfrak{B}_{\mathcal{D}}^{\ell, \hat{\ell}}(f)=\hat{\ell}\left(\Lambda_{\mathcal{D}}(\ell \circ \pi(f))\right) .
$$

This is a distribution in $f \in \mathcal{S}(\mathrm{G})$, which we call the Bessel distribution (with respect to $\mathcal{D}, \ell, \hat{\ell})$.

If $\mathcal{D}=(\pi, \hat{\pi},(\cdot, \cdot))$ is a dual couple then we define the opposite dual couple to be $\mathcal{D}^{\circ}=\left(\hat{\pi}, \pi,(\cdot, \cdot)^{\circ}\right)$ where $(\hat{v}, v)^{\circ}=(v, \hat{v})$. If $\chi$ is a character of $\mathrm{E}^{*}$ (viewed as a character of $\mathrm{G}$ through det) then $(\cdot, \cdot)$ also gives a duality between $\pi \cdot \chi$ and $\hat{\pi} \cdot \chi^{-1}$. We write $\mathcal{D} \cdot \chi=\left(\pi \cdot \chi, \hat{\pi} \cdot \chi^{-1},(\cdot, \cdot)\right)$.

Given a dual couple $\mathcal{D}=(\sigma, \hat{\sigma},(\cdot, \cdot))$ with respect to $\mathrm{M}$ we can define the induced couple $\mathrm{I}(\mathcal{D}, \lambda)=(\mathrm{I}(\sigma, \lambda), \mathrm{I}(\hat{\sigma},-\lambda), \mathrm{I}(\cdot, \cdot))$ for any $\lambda \in \mathfrak{a}_{\mathrm{M}, \mathbf{C}}^{*}$ where

$$
(\varphi, \hat{\varphi})_{\mathrm{I}(\cdot, \cdot)}=\int_{\mathrm{P} \backslash \mathrm{G}}(\varphi(g), \hat{\varphi}(g)) d g .
$$

(This depends implicitly on $\psi$ through the measure on $P \backslash G$.)

We record some simple formal properties of Bessel distributions in the following Lemma.

Lemma 2.1. - Let $\mathcal{D}=(\pi, \hat{\pi},(\cdot, \cdot))$ be a dual couple and $f \in \mathcal{S}(\mathrm{G})$. Then

(1) Let $^{\vee}(g)=f\left(g^{-1}\right)$. Then

$$
\mathfrak{B}_{\mathcal{D}}^{\ell, \hat{\ell}}(f)=\mathfrak{B}_{\mathcal{D}^{\circ}}^{\hat{\ell}, \ell}\left(f^{\vee}\right) .
$$

(2) For any $g, g^{\prime} \in \mathrm{G}$ we have

$$
\mathfrak{B}_{\mathcal{D}}^{\ell \circ \pi(g), \hat{\ell} \circ \hat{\pi}\left(g^{\prime}\right)}(f)=\mathfrak{B}_{\mathcal{D}}^{\ell, \hat{\ell}}\left(\mathrm{L}(g) \mathbf{R}\left(g^{\prime}\right) f\right)
$$

(3) Similarly, for any $f_{1}, f_{2} \in \mathcal{S}(\mathrm{G})$ we have

$$
\mathfrak{B}_{\mathcal{D}}^{\ell \circ \pi\left(f_{1}\right), \hat{\ell} \circ \hat{\pi}\left(f_{2}\right)}(f)=\mathfrak{B}_{\mathcal{D}}^{\ell, \hat{\ell}}\left(f_{1} * f * f_{2}^{\vee}\right) .
$$

(4) For any character $\chi$ of $\mathrm{E}^{*}$ we have

$$
\mathfrak{B}_{\mathcal{D} \cdot \chi}^{\ell, \hat{\ell}}(f)=\mathfrak{B}_{\mathcal{D}}^{\ell, \hat{\ell}}(f \cdot \chi) .
$$


(5) If $(\mathrm{A}, \hat{\mathrm{A}}): \mathcal{D}_{1} \rightarrow \mathcal{D}_{2}=\left(\pi_{2}, \hat{\pi}_{2},(\cdot, \cdot)_{2}\right)$ is an equivalence of dual couples, $\ell \in \pi_{2}^{*}$ and $\hat{\ell} \in \hat{\pi}_{2}^{*}$ then

$$
\mathfrak{B}_{\mathcal{D}_{1}, \hat{\ell}^{\ell} \hat{A}}^{\ell}=\mathfrak{B}_{\mathcal{D}_{2}}^{\ell, \hat{\ell}} .
$$

(6) Suppose that $\pi \in \Pi_{\mathrm{unr}}$ and let $v_{0}$ and $\hat{v}_{0}$ be non-zero spherical vectors in $\pi$ and $\hat{\pi}$ respectively. Then for any bi-K-invariant $f$ we have

$$
\mathfrak{B}_{\mathcal{D}}^{\ell, \hat{\ell}}(f)=\hat{f}(\pi) \frac{\ell\left(v_{0}\right) \hat{\ell}\left(\hat{v}_{0}\right)}{\left(v_{0}, \hat{v}_{0}\right)} .
$$

We say that $\ell \in \pi^{*}$ is injective if for every non-zero $v \in \pi$ there exists $g \in \mathrm{G}$ such that $\ell(\pi(g) v) \neq 0$. In other words, the intertwining map $\pi \rightarrow \mathrm{C}^{\infty}(\mathrm{G})$ given by the generalized matrix coefficients defined by $\ell$ is injective. This is automatic if $\pi \in \Pi$ and $\ell \neq 0$.

\section{Lemma 2.2.}

1. Suppose that $\mathcal{D}=(\pi, \hat{\pi},(\cdot, \cdot))$ is a dual couple, $0 \neq \ell \in \pi^{*}$ and $\hat{\ell} \in \hat{\pi}^{*}$ is injective. Then $\mathfrak{B}_{\mathcal{D}}^{\ell, \hat{\ell}} \neq 0$.

2. Suppose that $\mathcal{D}_{i}=\left(\pi_{i}, \hat{\pi}_{i},(\cdot, \cdot)_{i}\right), i=1, \ldots, r$ are dual couples with pairwise inequivalent $\pi_{1}, \ldots, \pi_{r} \in \Pi$. Let $0 \neq \ell_{i} \in \pi_{i}^{*}, 0 \neq \hat{\ell}_{i} \in \hat{\pi}_{i}^{*}, i=1, \ldots, r$. Then the Bessel distributions $\mathfrak{B}_{\mathcal{D}_{i}}^{\ell_{i}, \hat{\ell}_{i}}, i=1, \ldots$, r are linearly independent.

Proof. - To show the first part, take any $f \in \mathcal{S}(\mathrm{G})$ such that $v^{\vee}:=\ell \circ \pi(f) \in \pi^{\vee}$ is non-zero. Since $\hat{\ell} \circ \Lambda_{\mathcal{D}} \in \pi^{\vee *}$ is injective, there exists $g \in \mathrm{G}$ such that $\mathfrak{B}_{\mathcal{D}}^{\ell, \hat{\ell}}(\mathrm{R}(g) f)=$ $\hat{\ell}\left(\Lambda_{\mathcal{D}}\left(\pi^{\vee}(g) v^{\vee}\right)\right) \neq 0$.

To show the second part, assume that $\sum_{i=1}^{r} a_{i} \mathfrak{B}_{\mathcal{D}_{i}}^{\ell_{i}, \hat{\ell}_{i}}=0$ with $a_{i} \in \mathbf{C}$. Let $\mathcal{D}=$ $(\pi, \hat{\pi},(\cdot, \cdot))=\bigoplus \mathcal{D}_{i}, \ell=\bigoplus_{i=1}^{r} a_{i} \ell_{i} \in \pi^{*}$ and $\hat{\ell}=\bigoplus_{i=1}^{r} \hat{\ell}_{i} \in \hat{\pi}^{*}$. Then $\hat{\ell}$ is injective since $\pi_{i}$ are irreducible and pairwise inequivalent and $\mathfrak{B}_{\mathcal{D}}^{\ell, \hat{\ell}}=\sum_{i=1}^{r} a_{i} \mathfrak{B}_{\mathcal{D}_{i}}^{\ell_{i}, \hat{\ell}_{i}}=0$. By the first part, $\ell=0$ and therefore $a_{i}=0$ for all $i$.

Suppose now that $\pi$ is a unitary representation of $\mathrm{G}$ on a Hilbert space $(\mathfrak{H},(\cdot, \cdot))$. Let $\mathrm{V}$ be the space of smooth vectors in $\mathfrak{H}$. Assume that $\mathrm{V}$ is of finite length. Let $\hat{\pi}$ be the representation on the conjugate Hilbert space $\hat{\mathfrak{H}}$ and let $\hat{\mathrm{V}} \subseteq \hat{\mathfrak{H}}$ be the image of $\mathrm{V}$ under the canonical conjugate-linear automorphism $\mathfrak{H} \rightarrow \hat{\mathfrak{H}}$. Note that $\hat{\mathrm{V}}$ coincides with the space of smooth vectors of $\hat{\pi}$. Let $\mathcal{D}=(\pi, \hat{\pi},(\cdot, \cdot))$ so that $\Lambda_{\mathcal{D}}: \pi^{\vee} \rightarrow \hat{\pi}$ is the restriction of the canonical isomorphism $\mathfrak{H}^{*} \rightarrow \hat{\mathfrak{H}}$. We say that an orthonormal basis $\left\{e_{i}\right\}$ of $\mathfrak{H}$ is admissible if it is contained in $\mathrm{V}$ and for any $v \in \mathrm{V}$ we have $v=\sum_{i}\left(v, e_{i}\right) e_{i}$ where the sum has only finitely many non-zero terms in the $p$-adic case and $\sum_{i}\left|\left(v, e_{i}\right)\right| \mu\left(e_{i}\right)$ converges for any continuous seminorm $\mu$ in the Archimedean case. For instance, the union over $\gamma \in \hat{\mathrm{K}}$ of orthonormal bases of $\mathrm{V}^{\gamma}$ is an admissible orthonormal basis. Suppose that $\left\{e_{i}\right\}$ 
is an admissible orthonormal basis of $\mathfrak{H}$. We write $\left\{\hat{e}_{i}\right\}$ for $\left\{e_{i}\right\}$ viewed as an orthonormal basis of $\hat{\mathfrak{H}}$. Then for any $\ell \in \mathrm{V}^{*}, \hat{\ell} \in \hat{\mathrm{V}}^{*}$ we have

$$
\mathfrak{B}_{\mathcal{D}}^{\ell, \hat{\ell}}(f)=\sum_{i} \ell\left(\pi(f) e_{i}\right) \hat{\ell}\left(\hat{e}_{i}\right)
$$

where the sum converges absolutely. Indeed, $\left\{\hat{e}_{i}\right\}$ is also admissible so that $\Lambda_{\mathcal{D}}[\ell \circ \pi(f)]=$ $\sum_{i} \ell\left(\pi(f) e_{i}\right) \hat{e}_{i}$ in $\hat{V}$. Applying $\hat{\ell}$ we get the above.

Consider now a family of induced representations. Let $\sigma \in \mathcal{R}(\mathrm{M})$ and let $\mathrm{V}=$ $\mathrm{I}(\sigma)$. The map $\mathfrak{a}_{\mathrm{M}, \mathbf{G}}^{*} \times \mathrm{G} \times \mathrm{V} \rightarrow \mathrm{V}$ given by $(\lambda, g, v) \mapsto \mathrm{I}(g, \sigma, \lambda) v$ is continuous, and is analytic in $\lambda$. In addition, in the Archimedean case, for any compact set $\mathrm{C} \subseteq \mathfrak{a}_{\mathrm{M}, \mathbf{G}}^{*}$ and a continuous seminorm $v$ on $\mathrm{V}$ there exists $\mathrm{N}$ such that

$$
v \mapsto \sup _{\lambda \in \mathrm{C}, g \in \mathrm{G}} \frac{v(\mathrm{I}(g, \sigma, \lambda) v)}{\|g\|^{\mathrm{N}}}
$$

is a continuous seminorm on $\mathrm{V}$, where $\|g\|=\max \left(\left|g_{i, j}\right|,|\operatorname{det} g|^{-1}\right)$. It easily follows that for any $f \in \mathcal{S}(\mathrm{G})$ and $v \in \mathrm{V}$ the map $\mathfrak{a}_{\mathrm{M}, \mathbf{G}}^{*} \rightarrow \mathrm{V}$ defined by $\lambda \mapsto \mathrm{I}(f, \sigma, \lambda) v$ is analytic. Moreover, for any $\ell \in \mathrm{V}^{*}$ the map $\lambda \mapsto \ell \circ \mathrm{I}(f, \sigma, \lambda) \in \mathrm{V}^{\vee}$ is analytic.

We say that a family $\ell(\lambda) \in \mathrm{I}(\sigma, \lambda)^{*}, \lambda \in \mathfrak{a}_{\mathrm{M}, \mathbf{C}}^{*}$ is meromorphic if for any $\lambda_{0} \in \mathfrak{a}_{\mathrm{M}, \mathbf{C}}^{*}$ there exists a connected neighborhood $\mathrm{U}$ and a non-zero analytic function $p: \mathrm{U} \rightarrow \mathbf{C}$ such that for all $v \in \mathrm{V}$ the function $p(\lambda) \ell(\lambda)(v)$ is analytic on $\mathrm{U}$.

Lemma 2.3. - Let $\mathcal{D}=(\sigma, \hat{\sigma},(\cdot, \cdot))$ be a dual couple with respect to $\mathrm{M}$ and let $\ell(\lambda)$ (resp. $\hat{\ell}(\lambda)$ ) be a meromorphic family in $\mathrm{I}(\sigma, \lambda)^{*}$ (resp. $\left.\mathrm{I}(\hat{\sigma},-\lambda)^{*}\right)$. Then for any $f \in \mathcal{S}(\mathrm{G})$ the expression $\mathrm{D}_{\lambda}(f)=\mathfrak{B}_{\mathrm{I}(\mathcal{D}, \lambda)}^{\ell(\lambda), \hat{\ell}(\lambda)}(f)$ is a meromorphic function of $\lambda$. Suppose further that $\mathrm{D}_{\lambda}$ is holomorphic at $\lambda_{0}$ (i.e., for every $f \in \mathcal{S}(\mathrm{G}), \mathrm{D}_{\lambda}(f)$ is holomorphic at $\left.\lambda_{0}\right), \hat{\ell}$ is holomorphic at $\lambda_{0}$ and $\hat{\ell}\left(\lambda_{0}\right) \in \mathrm{I}\left(\hat{\sigma},-\lambda_{0}\right)^{*}$ is injective. Then $\ell(\lambda)$ is also holomorphic at $\lambda=\lambda_{0}$.

Proof. - For the first part we may assume that $\ell(\lambda)$ and $\hat{\ell}(\lambda)$ are analytic. By the remarks above, the expression

$$
\hat{\ell}\left(\lambda_{2}\right) \circ \Lambda_{\mathrm{I}(\mathcal{D})}\left[\ell\left(\lambda_{1}\right) \circ \mathrm{I}\left(f, \sigma, \lambda_{3}\right)\right]=\ell\left(\lambda_{1}\right) \circ \Lambda_{\mathrm{I}\left(\mathcal{D}^{\circ}\right)}\left[\hat{\ell}\left(\lambda_{2}\right) \circ \mathrm{I}\left(f^{\vee}, \hat{\sigma},-\lambda_{3}\right)\right]
$$

is separately holomorphic in $\lambda_{1}, \lambda_{2}, \lambda_{3}$. Therefore, by Hartogs' Theorem, it is jointly holomorphic in $\lambda_{1}, \lambda_{2}, \lambda_{3}$, and in particular, holomorphic for $\lambda_{1}=\lambda_{2}=\lambda_{3}=\lambda$.

We prove the second part. Assume on the contrary that $\ell(\lambda)$ is not holomorphic at $\lambda=\lambda_{0}$. Then for some generic direction $\mu \in \mathfrak{a}_{\mathrm{M}, \mathbf{G}}^{*}$, the linear form $\ell\left(\lambda_{0}+s \mu\right)$ is a meromorphic function in $s$ which is holomorphic at some punctured disc centered at $s=0$ and has a pole at $s=0$. Therefore, there exists $m>0$ such that $s^{m} \ell\left(\lambda_{0}+s \mu\right)$ is holomorphic near $s=0$ and its value $\mathrm{L}$ at $s=0$ is a non-zero element of $\mathrm{V}^{*}$. However, for any $f \in \mathcal{S}(\mathrm{G})$ 


$$
\mathfrak{B}_{\mathrm{I}\left(\mathcal{D}, \lambda_{0}\right)}^{\mathrm{L}, \hat{\ell}\left(\lambda_{0}\right)}(f)=\left[s^{m} \mathrm{D}_{\lambda_{0}+s \mu}(f)\right]_{s=0}=0
$$

since $\mathrm{D}_{\lambda}$ is holomorphic at $\lambda=\lambda_{0}$. This contradicts Lemma 2.2. The lemma follows.

2.1.1. Next we consider relative Bessel distributions on the symmetric space $X$. Let

$$
\mathcal{E}_{\mathrm{G}}\left(\mathrm{X}, \pi^{*}\right)=\left\{\alpha: \mathrm{X} \rightarrow \pi^{*}: \alpha_{x \bullet g}=\alpha_{x} \circ \pi(g) \text { for all } g \in \mathrm{G} \text { and } x \in \mathrm{X}\right\} .
$$

Here, the value of $\alpha$ at $x \in \mathrm{X}$ is denoted by $\alpha_{x}$. In particular, if $\alpha \in \mathcal{E}_{\mathrm{G}}\left(\mathrm{X}, \pi^{*}\right)$ then $\alpha_{x} \in \operatorname{Hom}_{\mathrm{G}^{x}}(\pi, \mathbf{G})$ for all $x \in \mathrm{X}$.

Definition 2.4. - We say that $\pi \in \mathcal{R}(\mathrm{G})$ is $\mathrm{G}^{x}$-distinguished if $\operatorname{Hom}_{\mathrm{G}^{x}}(\pi, \mathbf{G}) \neq 0$. The elements of $\operatorname{Hom}_{\mathrm{G}^{x}}(\pi, \mathbf{G})$ are called (local) unitary periods.

Thus, an element of $\mathcal{E}_{\mathrm{G}}\left(\mathrm{X}, \pi^{*}\right)$ is a compatible family of local unitary periods. More precisely, for every $x \in \mathrm{X}$ and $g \in \mathrm{G}$ the map $\ell \mapsto \ell \circ \pi(g)$ defines an isomorphism

$$
\left(\pi^{*}\right)^{\mathrm{G}^{x}}=\operatorname{Hom}_{\mathrm{G}^{x}}(\pi, \mathbf{G}) \simeq \operatorname{Hom}_{\mathrm{G}^{x \bullet g}}(\pi, \mathbf{G})=\left(\pi^{*}\right)^{\mathrm{G}^{x \bullet g}} .
$$

With these identifications, the map $\alpha \mapsto\left(\alpha_{x}\right)_{x \in \mathrm{X} / \mathrm{G}}$ is an isomorphism

$$
\mathcal{E}_{\mathrm{G}}\left(\mathrm{X}, \pi^{*}\right) \simeq \bigoplus_{x \in \mathrm{X} / \mathrm{G}}\left(\pi^{*}\right)^{\mathrm{G}^{x}} .
$$

We can view $\mathcal{E}_{\mathrm{G}}\left(\mathrm{X}, \pi^{*}\right)$ slightly differently as follows. For any $\alpha \in \mathcal{E}_{\mathrm{G}}\left(\mathrm{X}, \pi^{*}\right)$ and $\Phi \in$ $\mathcal{S}(\mathrm{X})$, let $\Phi \odot \alpha \in \pi^{\vee}$ be given by

$$
\Phi \odot \alpha(v)=\int_{\mathrm{X}} \Phi(x) \alpha_{x}(v) d x, \quad \Phi \in \mathcal{S}(\mathrm{X}), v \in \pi .
$$

Then the map

$$
\alpha \mapsto(\Phi \mapsto \Phi \odot \alpha)
$$

defines an isomorphism of vector spaces $\mathcal{E}_{\mathrm{G}}\left(\mathrm{X}, \pi^{*}\right) \rightarrow \operatorname{Hom}_{\mathrm{G}}\left(\mathcal{S}(\mathrm{X}), \pi^{\vee}\right.$ ) (with the right action of $\mathrm{G}$ on $\mathcal{S}(\mathrm{X})$ ), i.e.,

$$
(f * \Phi) \odot \alpha=\pi^{\vee}(f)(\Phi \odot \alpha)
$$

for any $\Phi \in \mathcal{S}(\mathrm{X})$ and $f \in \mathcal{S}(\mathrm{G})$.

The above isomorphism is compatible with the identifications

$$
\begin{aligned}
\mathcal{E}_{\mathrm{G}}\left(\mathrm{X}, \pi^{*}\right) & \simeq \bigoplus_{x \in \mathrm{X} / \mathrm{G}}\left(\pi^{*}\right)^{\mathrm{G}^{x}} \simeq \bigoplus_{x \in \mathrm{X} / \mathrm{G}} \operatorname{Hom}_{\mathrm{G}}\left(\mathcal{S}\left(\mathrm{G}^{x} \backslash \mathrm{G}\right), \pi^{\vee}\right) \\
& \simeq \operatorname{Hom}_{\mathrm{G}}\left(\mathcal{S}(\mathrm{X}), \pi^{\vee}\right)
\end{aligned}
$$


where for the middle one, we use the identification

$$
\left(\pi^{*}\right)^{\mathrm{G}^{x}} \simeq\left[\operatorname{Hom}_{\mathrm{G}}\left(\mathcal{S}(\mathrm{G})^{r}, \pi^{\vee}\right)\right]^{\mathrm{G}^{x}} \simeq \operatorname{Hom}_{\mathrm{G}}\left(\mathcal{S}\left(\mathrm{G}^{x} \backslash \mathrm{G}\right), \pi^{\vee}\right)
$$

obtained by viewing $\mathcal{S}\left(\mathrm{G}^{x} \backslash \mathrm{G}\right)$ as the left $\mathrm{G}^{x}$-coinvariants of $\mathcal{S}(\mathrm{G})$.

If $\pi_{1}, \pi_{2} \in \mathcal{R}(\mathrm{G}), \mathrm{T}: \pi_{1} \rightarrow \pi_{2}$ is an intertwining operator and $\alpha \in \mathcal{E}_{\mathrm{G}}\left(\mathrm{X}, \pi_{2}^{*}\right)$ then we write $\alpha \circ \mathrm{T} \in \mathcal{E}_{\mathrm{G}}\left(\mathrm{X}, \pi_{1}^{*}\right)$ for the equivariant map $x \mapsto \alpha_{x} \circ \mathrm{T}$.

The relative Bessel distribution associated with a dual couple $\mathcal{D}=(\pi, \hat{\pi},(\cdot, \cdot))$, a linear form $\hat{\ell} \in \hat{\pi}^{*}$ and $\alpha \in \mathcal{E}_{\mathrm{G}}\left(\mathrm{X}, \pi^{*}\right)$ is defined by

$$
\tilde{\mathfrak{B}}_{\mathcal{D}}^{\alpha, \hat{\ell}}(\Phi)=\hat{\ell}\left(\Lambda_{\mathcal{D}}(\Phi \odot \alpha)\right), \quad \Phi \in \mathcal{S}(\mathrm{X}) .
$$

As before, for any $g \in \mathrm{G}$ we have

$$
\tilde{\mathfrak{B}}_{\mathcal{D}}^{\alpha, \hat{\ell} \circ \hat{\pi}(g)}(\Phi)=\tilde{\mathfrak{B}}_{\mathcal{D}}^{\alpha, \hat{\ell}}(\mathrm{R}(g) \Phi)
$$

where $\mathrm{R}(g) \Phi(x)=\Phi(x \bullet g)$. Similarly, for any $f \in \mathcal{S}(\mathrm{G})$ we have

$$
\tilde{\mathfrak{B}}_{\mathcal{D}}^{\alpha, \hat{\ell} \circ \hat{\pi}(f)}(\Phi)=\tilde{\mathfrak{B}}_{\mathcal{D}}^{\alpha, \hat{\ell}}(f * \Phi) .
$$

Moreover, if $(\mathrm{A}, \hat{\mathrm{A}}): \mathcal{D}_{1} \rightarrow \mathcal{D}_{2}=\left(\pi_{2}, \hat{\pi}_{2},(\cdot, \cdot)_{2}\right)$ is an equivalence of dual couples, $\alpha \in \mathcal{E}_{\mathrm{G}}\left(\mathrm{X}, \pi_{2}^{*}\right)$ and $\hat{\ell} \in \pi_{2}^{*}$ then we have

$$
\tilde{\mathfrak{B}}_{\mathcal{D}_{1}}^{\alpha \circ A, \hat{\ell} \circ \hat{A}}=\tilde{\mathfrak{B}}_{\mathcal{D}_{2}}^{\alpha, \hat{\ell}} .
$$

Finally, if $\pi \in \Pi_{\mathrm{unr}}, \mathrm{F}$ is $p$-adic, $\mathrm{E} / \mathrm{F}$ is unramified and $v_{0}$ and $\hat{v}_{0}$ are non-zero spherical vectors in $\pi$ and $\hat{\pi}$ respectively, then

$$
\tilde{\mathfrak{B}}_{\mathcal{D}}^{\alpha, \hat{\ell}}\left(f^{\vee} * \mathbf{1}_{\mathrm{X} \cap \mathrm{K}}\right)=\operatorname{vol}_{\mathrm{X}}(\mathrm{X} \cap \mathrm{K}) \hat{f}(\pi) \frac{\alpha_{e}\left(v_{0}\right) \hat{\ell}\left(\hat{v}_{0}\right)}{\left(v_{0}, \hat{v}_{0}\right)}
$$

for any bi-K-invariant $f$.

The relative Bessel distribution on $\mathrm{X}$ can be expressed as a sum over the G-orbits in $\mathrm{X}$ of Bessel distributions on $\mathrm{G}$ as follows. For any $\Phi \in \mathcal{S}(\mathrm{X})$ and $x \in \mathrm{X}$ there exists $f \in \mathcal{S}(\mathrm{G})$ such that

$$
\Phi(x \bullet g)=\int_{\mathrm{G}^{x}} f(h g) d h, \quad g \in \mathrm{G} .
$$

In this case, we say that $\Phi$ is represented by $f$ (or that $f$ represents $\Phi$ ) at $x$. We say that the family $f^{x} \in \mathcal{S}(\mathrm{G}), x \in \mathrm{X}$ represents $\Phi$ if $f^{x}$ represents $\Phi$ at $x$ for all $x$. In this case, for any $v \in \pi$ we have

$$
(\Phi \odot \alpha)(v)=\sum_{x \in \mathrm{X} / \mathrm{G}} \int_{\mathrm{G}^{x} \backslash \mathrm{G}} \Phi(x \bullet g) \alpha_{x \bullet g}(v) d g
$$




$$
\begin{aligned}
& =\sum_{x \in \mathrm{X} / \mathrm{G}} \int_{\mathrm{G}^{x} \backslash \mathrm{G}} \int_{\mathrm{G}^{x}} f^{x}(h g) \alpha_{x}(\pi(g) v) d h d g \\
& =\sum_{x \in \mathrm{X} / \mathrm{G}} \int_{\mathrm{G}} f^{x}(g) \alpha_{x}(\pi(g) v) d g=\sum_{x \in \mathrm{X} / \mathrm{G}} \alpha_{x}\left(\pi\left(f^{x}\right) v\right) .
\end{aligned}
$$

In particular, $\alpha_{x} \circ \pi\left(f^{x}\right)$ does not depend on the choice of $f^{x}$ nor on the representative $x$. It follows that

$$
\tilde{\mathfrak{B}}_{\mathcal{D}}^{\alpha, \hat{\ell}}(\Phi)=\sum_{x \in \mathrm{X} / \mathrm{G}} \mathfrak{B}_{\mathcal{D}}^{\alpha_{x}, \hat{\ell}}\left(f^{x}\right) .
$$

We conclude from Lemma 2.2 that

$$
\text { if } \pi \in \Pi \text { and } \alpha \text { and } \hat{\ell} \text { are non-zero then } \tilde{\mathfrak{B}}_{\mathcal{D}}^{\alpha, \hat{\ell}} \text { is non-zero. }
$$

2.2. Normalized Bessel distributions. - Define the Whittaker couple $\mathfrak{W}(\pi)$ associated to $\pi \in \Pi_{\text {gen }}$ by $\mathfrak{W}(\pi)=\left(\mathcal{W}^{\psi}(\pi), \mathcal{W}^{\psi^{-1}}\left(\pi^{\vee}\right),[\cdot, \cdot]_{\pi}^{\psi}\right)$ where the pairing $[\cdot, \cdot]=[\cdot, \cdot]_{\pi}^{\psi}$ is defined in Appendix A. It will be useful to adopt the following notational convention. Whenever an object is related to a contragredient representation (on $\mathrm{G}$ or $\mathrm{M}$ ), the additive character which is used to define it is implicitly assumed to be $\psi^{-1}$ (rather than $\psi$ ). Analogously for $\mathrm{G}^{\prime}$ and $\mathrm{M}^{\prime}$. Thus, $\mathcal{W}\left(\pi^{\vee}\right)=\mathcal{W}^{\psi^{-1}}\left(\pi^{\vee}\right), \mathbf{W}_{e}\left(\sigma^{\vee}, \lambda\right)=\mathbf{W}_{e}^{\psi^{-1}}\left(\sigma^{\vee}, \lambda\right)$, etc. Hopefully this abuse of notation will not create any ambiguity. to $\pi^{\prime}$ by

Given $\pi^{\prime} \in \Pi_{\text {gen }}^{\prime}$, we define the normalized local Bessel distribution $\mathbf{B}_{\pi^{\prime}}$ on $\mathrm{G}^{\prime}$ associated

$$
\mathbf{B}_{\pi^{\prime}}\left(f^{\prime}\right)=\mathfrak{B}_{\mathfrak{W}\left(\pi^{\prime}\right)}^{\delta_{w_{0}^{\prime}}^{\pi^{\prime}}, \delta^{\pi^{\prime}}}\left(f^{\prime}\right)
$$

By (2.1), $\mathbf{B}_{\pi^{\prime}}\left(f^{\prime}\right)$ belongs to the space $\mathcal{S}^{*}\left(\mathrm{G}^{\prime}\right)^{\left({ }^{t} \mathrm{U}_{0}^{\prime} \times \mathrm{U}_{0}^{\prime},{ }^{t} \psi_{0}^{\prime} \times \psi_{0}^{\prime-1}\right)}$ of distributions $\mathrm{D}^{\prime}$ on $\mathrm{G}^{\prime}$ satisfying

$$
\begin{gathered}
\mathrm{D}^{\prime}\left(\mathrm{L}\left(u_{1}\right) \mathrm{R}\left(u_{2}\right) f^{\prime}\right)={ }^{t} \psi_{0}^{\prime}\left(u_{1}\right) \psi_{0}^{\prime}\left(u_{2}\right)^{-1} \mathrm{D}^{\prime}\left(f^{\prime}\right) \\
\text { for any } f^{\prime} \in \mathcal{S}\left(\mathrm{G}^{\prime}\right), u_{1} \in{ }^{t} \mathrm{U}_{0}^{\prime}, u_{2} \in \mathrm{U}_{0}^{\prime}
\end{gathered}
$$

where ${ }^{t} \psi_{0}^{\prime}$ is the character on ${ }^{t} \mathrm{U}_{0}^{\prime}$ given by ${ }^{t} \psi_{0}^{\prime}\left({ }^{t} u\right)=\psi_{0}^{\prime}(u), u \in \mathrm{U}_{0}^{\prime}$. tions

More generally, for any $\sigma^{\prime} \in \Pi_{\text {gen }}^{\mathrm{M}^{\prime}}$ define a holomorphic family of Bessel distribu-

$$
\mathbf{B}\left(f^{\prime}: \sigma^{\prime}, \lambda\right)=\mathfrak{B}_{\mathrm{I}\left(\mathfrak{W}\left(\sigma^{\prime}\right), \lambda\right)}^{\mathbf{W}\left(w_{0}: \sigma^{\prime}, \lambda\right), \mathbf{W}_{e}\left(\sigma^{\prime \vee},-\lambda\right)}\left(f^{\prime}\right) \quad f^{\prime} \in \mathcal{S}\left(\mathrm{G}^{\prime}\right), \lambda \in \mathfrak{a}_{\mathrm{M}, \mathbf{G}}^{*}
$$

By Proposition A.2, we have an equivalence of dual couples

$$
\Omega\left(\sigma^{\prime}, \lambda\right)=\left(\mathbf{W}\left(\sigma^{\prime}, \lambda\right), \mathbf{W}\left(\sigma^{\prime \vee},-\lambda\right)\right): \mathrm{I}\left(\mathfrak{W}\left(\sigma^{\prime}\right), \lambda\right) \rightarrow \mathfrak{W}\left(\mathrm{I}\left(\sigma^{\prime}, \lambda\right)\right)
$$

whenever $\mathrm{I}\left(\sigma^{\prime}, \lambda\right)$ is irreducible. 
Applying the relation (2.4), we infer that

$$
\mathbf{B}\left(\sigma^{\prime}, \lambda\right)=\mathbf{B}_{\mathrm{I}\left(\sigma^{\prime}, \lambda\right)}
$$

provided that $\mathrm{I}\left(\sigma^{\prime}, \lambda\right) \in \Pi^{\prime}$.

Remark 2.5. - It is likely that even if $\mathrm{I}\left(\sigma^{\prime}, \lambda\right)$ is reducible, we still have $\mathbf{B}\left(\sigma^{\prime}, \lambda\right)=$ $\mathbf{B}_{\pi^{\prime}}$ where $\pi^{\prime}$ is the generic subquotient of $\mathrm{I}\left(\sigma^{\prime}, \lambda\right)$. However, we will not discuss this question here.

Let $\chi^{\prime}$ be a character of $\mathrm{F}^{*}$. We also view $\chi^{\prime}$ as a character of $\mathrm{G}^{\prime}$ via the determinant. For any function $f^{\prime}$ on $\mathrm{G}^{\prime}$ set $f_{\chi^{\prime}}^{\prime}(g)=\chi^{\prime}(g) f^{\prime}(g), g \in \mathrm{G}^{\prime}$. For $\pi^{\prime} \in \Pi_{\text {gen }}^{\prime}$ let $\mathrm{A}_{\chi^{\prime}}^{\pi^{\prime}}$ : $\mathcal{W}\left(\pi^{\prime}\right) \cdot \chi^{\prime} \rightarrow \mathcal{W}\left(\pi^{\prime} \cdot \chi^{\prime}\right)$ be the isomorphism defined by $\mathrm{A}_{\chi^{\prime}}^{\pi^{\prime}}(\mathrm{W})=\mathrm{W}_{\chi^{\prime}}, \mathrm{W} \in \mathcal{W}\left(\pi^{\prime}\right)$. Then by $(\mathbf{A . 3}),\left(\mathrm{A}_{\chi^{\prime}}^{\pi^{\prime}}, \mathrm{A}_{\chi^{\prime-1}}^{\pi^{\prime \prime}}\right): \mathfrak{W}\left(\pi^{\prime}\right) \cdot \chi^{\prime} \rightarrow \mathfrak{W}\left(\pi^{\prime} \cdot \chi^{\prime}\right)$ is an equivalence. Thus, by $(\mathbf{2 . 4})$ we get

$$
\chi^{\prime}\left(w_{0}\right) \mathfrak{B}_{\mathfrak{W}\left(\pi^{\prime}\right) \cdot \chi^{\prime}}^{\delta_{w_{0}^{\prime}}^{\pi^{\prime}}, \delta_{l}^{\pi^{\prime}}}=\mathbf{B}_{\pi^{\prime} \cdot \chi^{\prime}}
$$

Combined with (2.3), we infer that

$$
\mathbf{B}_{\pi^{\prime} \cdot \chi^{\prime}}\left(f^{\prime}\right)=\chi^{\prime}\left(w_{0}\right) \mathbf{B}_{\pi^{\prime}}\left(f_{\chi^{\prime}}^{\prime}\right)
$$

for any $f^{\prime} \in \mathcal{S}\left(\mathrm{G}^{\prime}\right)$.

Assume now that $\mathrm{E} / \mathrm{F}$ is inert. Let

$$
\mathrm{G}^{\prime \pm}=\left\{g \in \mathrm{G}^{\prime}: \eta\left(w_{0} g\right)= \pm 1\right\}
$$

so that $\mathrm{G}^{\prime}$ is the disjoint union of the open cosets $\mathrm{G}^{\prime \pm}$. Thus,

$$
\mathcal{S}\left(\mathrm{G}^{\prime}\right)=\mathcal{S}\left(\mathrm{G}^{\prime+}\right) \oplus \mathcal{S}\left(\mathrm{G}^{\prime-}\right)
$$

and

$$
\mathcal{S}\left(\mathrm{G}^{\prime \pm}\right)=\left\{f \in \mathcal{S}\left(\mathrm{G}^{\prime}\right): f_{\eta}= \pm \eta\left(w_{0}\right) f\right\}
$$

The following property of the support of the Bessel distribution will be useful for us.

Lemma 2.6. - For any $\pi^{\prime} \in \Pi_{\text {gen }}^{\prime}$ we have $\left.\mathbf{B}_{\pi^{\prime}}\right|_{\mathcal{S}\left(\mathrm{G}^{\prime+}\right)} \not \equiv 0$. Moreover, $\left.\mathbf{B}_{\pi^{\prime}}\right|_{\mathcal{S}\left(\mathrm{G}^{\prime}\right)} \equiv 0$ if and only if $\pi^{\prime} \simeq \pi^{\prime} \cdot \eta$.

Proof. - If $\pi^{\prime} \simeq \pi^{\prime} \cdot \eta$ then it follows from $(\mathbf{2 . 1 5})$ and $(\mathbf{2 . 1 6})$ that $\left.\mathbf{B}_{\pi^{\prime}}\right|_{\mathcal{S}\left(\mathrm{G}^{\prime}\right)} \equiv 0$. Since $\mathbf{B}_{\pi^{\prime}} \not \equiv 0$, it remains to see that if $\pi^{\prime} \not \pi^{\prime} \cdot \eta$ then $\mathbf{B}_{\pi^{\prime}}$ is non-zero on each of $\mathcal{S}\left(\mathrm{G}^{\prime \pm}\right)$. Since $\mathbf{B}_{\pi^{\prime}}$ and $\mathbf{B}_{\pi^{\prime} \cdot \eta}$ are linearly independent (Lemma 2.2) there exists $f^{\prime} \in$ 
$\mathcal{S}\left(\mathrm{G}^{\prime}\right)$ such that $\mathbf{B}_{\pi^{\prime}}\left(f^{\prime}\right) \neq 0$ and $\mathbf{B}_{\pi^{\prime} \cdot \eta}\left(f^{\prime}\right)=0$. By $(\mathbf{2 . 1 5}), \mathbf{B}_{\pi^{\prime}}\left(f_{\eta}^{\prime}\right)=0$ as well. Then $f_{ \pm}^{\prime}:=f^{\prime} \pm \eta\left(w_{0}\right) f_{\eta}^{\prime} \in \mathcal{S}\left(\mathrm{G}^{\prime \pm}\right)$ and $\mathbf{B}_{\pi^{\prime}}\left(f_{ \pm}^{\prime}\right)=\mathbf{B}_{\pi^{\prime}}\left(f^{\prime}\right) \neq 0$ as required.

Finally, suppose that $\mathrm{F}$ is $p$-adic, $\pi^{\prime} \in \Pi_{\text {gen,unr }}^{\prime}$ and $\psi^{\prime}$ has conductor $\mathcal{O}_{\mathrm{F}}$. Then by (A.4) and (2.5) we get

$$
\mathbf{B}_{\pi^{\prime}}\left(\mathbf{1}_{\mathrm{K}^{\prime}}\right)=\mathrm{L}\left(1, \pi^{\prime} \times \pi^{\prime \vee}\right)^{-1} .
$$

\section{Local identities of Bessel distributions}

In this section, we describe a family of $\mathrm{G}^{x}$-invariant linear forms characterized by an identity of local Bessel distributions for matching functions. These $\mathrm{G}^{x}$-invariant forms will eventually appear as the local factors of the global unitary period integral.

We first recall the definition of functions with matching orbital integrals.

3.1. Matching of orbital integrals. - Denote by $\gamma: \mathrm{M}_{0}^{\prime} \rightarrow\{ \pm 1\}$ the transfer factor defined by

$$
\gamma(a)=\eta\left(a_{1}\right) \eta^{2}\left(a_{2}\right) \ldots \eta^{n}\left(a_{n}\right), \quad a=\operatorname{diag}\left(a_{1}, \ldots, a_{n}\right) \in \mathrm{M}_{0}^{\prime} .
$$

Note that this differs from the transfer factor defined by Jacquet in JJac05a] by a factor of $\eta^{n}(a)$. This is motivated by [Off09, Theorem 10.1].

In the inert case, we say that $\Phi \in \mathcal{S}(\mathrm{X})$ and $f^{\prime} \in \mathcal{S}\left(\mathrm{G}^{\prime}\right)$ have matching orbital integrals (or simply, $\Phi$ and $f^{\prime}$ match), and we write $\Phi \longleftrightarrow f^{\prime}$, if for every $a \in \mathrm{M}_{0}^{\prime}=\mathrm{X} \cap \mathrm{M}_{0}$ we have

$$
\gamma(a) \int_{\mathrm{U}_{0}^{\prime}} \int_{\mathrm{U}_{0}^{\prime}} f^{\prime}\left({ }^{t} u_{1} a u_{2}\right) \psi_{0}^{\prime}\left(u_{1} u_{2}\right) d u_{1} d u_{2}=\int_{\mathrm{U}_{0}} \Phi(a \bullet u) \psi_{0}(u) d u .
$$

Similarly, for any $x \in \mathrm{X}$ we say that $f \in \mathcal{S}(\mathrm{G})$ and $f^{\prime} \in \mathcal{S}\left(\mathrm{G}^{\prime}\right)$ have $x$-matching orbital integrals and we write $f \stackrel{x}{\longleftrightarrow} f^{\prime}$ if for every $a \in \mathbf{M}_{0}^{\prime}$ we have

$$
\begin{aligned}
& \gamma(a) \int_{\mathrm{U}_{0}^{\prime}} \int_{\mathrm{U}_{0}^{\prime}} f^{\prime}\left({ }^{t} u_{1} a u_{2}\right) \psi_{0}^{\prime}\left(u_{1} u_{2}\right) d u_{1} d u_{2} \\
& = \begin{cases}\int_{\mathrm{U}_{0}} \int_{\mathrm{G}^{x}} f(h g u) d h \psi_{0}(u) d u & \text { if } a=x \bullet g \text { for some } g \in \mathrm{G}, \\
0 & \text { if } a \notin x \bullet \mathrm{G} .\end{cases}
\end{aligned}
$$

In the split case, we write $\Phi \longleftrightarrow f^{\prime}$ if $\Phi\left({ }^{t} g, g\right)=f^{\prime}(g), g \in \mathrm{G}^{\prime}$, so that (3.1) is satisfied. Similarly, for any $f \in \mathcal{S}(\mathrm{G})$ and $h \in \mathrm{G}^{\prime}$ we write

$$
f \stackrel{\left(h,{ }^{h} h\right)}{\longleftrightarrow}\left(g^{\prime} \mapsto \int_{\mathrm{G}^{\prime}} f\left(y^{t} g^{\prime},{ }^{t}(h y)^{-1}\right) d y\right) .
$$

Once again (3.2) is satisfied. 
Back in the general case, we observe that for every $x \in \mathrm{X}$ and $g \in \mathrm{G}$ we have

$$
\mathrm{L}(g) f \stackrel{x}{\longleftrightarrow} f^{\prime} \quad \text { if and only if } f \stackrel{x \bullet g}{\longleftrightarrow} f^{\prime} .
$$

Let

$$
\mathrm{X}[x]=\{y \in \mathrm{X}: \eta(y)=\eta(x)\}
$$

and

$$
\mathrm{G}^{\prime}[x]=\left\{g \in \mathrm{G}^{\prime}: \eta(g)=\eta(x)\right\}
$$

In the split case, $\eta$ is trivial and therefore $\mathrm{X}[x]=\mathrm{X}$ and $\mathrm{G}^{\prime}[x]=\mathrm{G}^{\prime}$ for all $x \in \mathrm{X}$. In the inert case, we get a bisection of both $\mathrm{X}$ and $\mathrm{G}^{\prime}$ into two open subsets. In particular, $f^{\prime} \mathbf{1}_{\mathrm{G}^{\prime}[x]} \in \mathcal{S}\left(\mathrm{G}^{\prime}\right)$ whenever $f^{\prime} \in \mathcal{S}\left(\mathrm{G}^{\prime}\right)$. Note that for any $x \in \mathrm{X}$

$$
f \stackrel{x}{\longleftrightarrow} f^{\prime} \Longrightarrow f \stackrel{x}{\longleftrightarrow} f^{\prime} \mathbf{1}_{\mathrm{G}^{\prime}[x]}
$$

Observe that $\mathrm{X}[x]=x \bullet \mathrm{G}$ in the $p$-adic case, in the split case, and in the case where $\mathrm{E} / \mathrm{F}=\mathbf{C} / \mathbf{R}, n=2$ and $\mathrm{G}^{x}$ is quasi-split. Thus, in the split or non-Archimedean case, for $\Phi \in \mathcal{S}(\mathrm{X})$ represented by the family $\left(f^{x}\right)_{x \in \mathrm{X}}$ and $f^{\prime} \in \mathcal{S}\left(\mathrm{G}^{\prime}\right)$ we have

$$
\Phi \longleftrightarrow f^{\prime} \quad \text { if and only if } f^{x} \stackrel{x}{\longleftrightarrow} f^{\prime} \mathbf{1}_{\mathrm{G}^{\prime}[x]}, \forall x \in \mathrm{X}
$$

However, this is not true in the case $\mathbf{G} / \mathbf{R}$, for which there are $n+1$ orbits of $\mathrm{G}$ on $\mathbf{X}$, according to signature.

We recall the following key results

Theorem 3.1 (See [Jac03b, AG, Fac05a]).

- Smooth transfer: For every $\Phi \in \mathcal{S}(\mathrm{G})$ there exists $f^{\prime} \in \mathcal{S}\left(\mathrm{G}^{\prime}\right)$ and for every $f^{\prime} \in \mathcal{S}\left(\mathrm{G}^{\prime}\right)$ there exists $\Phi \in \mathcal{S}(\mathrm{X})$ such that $\Phi \longleftrightarrow f^{\prime}$. Thus, for every $x \in \mathrm{X}$ and $f \in \mathcal{S}(\mathrm{G})$ there exists $f^{\prime} \in \mathcal{S}\left(\mathrm{G}^{\prime}[x]\right)$ such that $f \stackrel{x}{\longleftrightarrow} f^{\prime}$. Conversely, if $\mathrm{X}[x]=x \bullet \mathrm{G}$ then for every $f^{\prime} \in$ $\mathcal{S}\left(\mathrm{G}^{\prime}[x]\right)$ there exists $f \in \mathcal{S}(\mathrm{G})$ such that $f \stackrel{x}{\longleftrightarrow} f^{\prime}$.

- The Fundamental Lemma: Assume that $\mathrm{F}$ is non-Archimedean of odd residual characteristic, $\mathrm{E} / \mathrm{F}$ is unramified and $\psi^{\prime}$ has conductor $\mathcal{O}_{\mathrm{F}}$. Suppose that $f \in \mathcal{S}(\mathrm{G})$ is bi-K-invariant and let $f^{\prime}$ be the bi-K'-invariant function that is the image off under the base change homomorphism of Hecke algebras. Then for $x \in \mathrm{X} \cap \mathrm{K}$ we have $f \stackrel{x}{\longleftrightarrow} f^{\prime}$. In particular

$$
\mathbf{1}_{\mathrm{K}} \stackrel{x}{\longleftrightarrow} \mathbf{1}_{\mathrm{K}^{\prime}}
$$


In the split case this is straightforward from the definitions. In the inert case, the first part was proved by Jacquet in the non-Archimedean case [Jac03b, Theorem 3] and by Aizenbud-Gourevitch in the Archimedean case [AG]. Note that the last assertion in the first part follows by taking $\Phi \longleftrightarrow f^{\prime}$ and any $f$ representing $\Phi$ at $x$.

Along with the relations (3.1), analogous relations for the singular orbital integrals are proved as well [Jac03a, AG].

The fundamental lemma was originally proved by Ngô in the case of positive characteristic, first for the unit element [Châ99] and then in general [Ngô99]. Later on, by a completely different approach, Jacquet gave a proof which is also valid in the characteristic zero case, first for the unit element [Jac04b] and then in general [Jac05a].

Let $\mathcal{S}^{*}(\mathrm{X})^{\left(\mathrm{U}_{0}, \psi_{0}^{-1}\right)}$ denote the space of distributions on $\mathrm{X}$ which are right $\left(\mathrm{U}_{0}, \psi_{0}^{-1}\right)$ equivariant. We say that $\mathrm{D} \in \mathcal{S}^{*}(\mathrm{X})^{\left(\mathrm{U}_{0}, \psi_{0}^{-1}\right)}$ and $\mathrm{D}^{\prime} \in \mathcal{S}^{*}\left(\mathrm{G}^{\prime}\right)^{\left({ }^{t} \mathrm{U}_{0}^{\prime} \times \mathrm{U}_{0}^{\prime},{ }^{t} \psi_{0}^{\prime} \times \psi_{0}^{\prime-1}\right)}$ match and we write $\mathrm{D} \longleftrightarrow \mathrm{D}^{\prime}$ if $\mathrm{D}(\Phi)=\mathrm{D}^{\prime}\left(f^{\prime}\right)$ whenever $\Phi \longleftrightarrow f^{\prime}$. In the $p$-adic case, it follows from smooth transfer and the principle of localization [GK75] that the relation $\mathrm{D} \longleftrightarrow \mathrm{D}^{\prime}$ defines an isomorphism of vector spaces

$$
\mathcal{S}^{*}(\mathrm{X})^{\left(\mathrm{U}_{0}, \psi_{0}^{-1}\right)} \simeq \mathcal{S}^{*}\left(\mathrm{G}^{\prime}\right)^{\left({ }^{t} \mathrm{U}_{0}^{\prime} \times \mathrm{U}_{0}^{\prime},{ }^{t} \psi_{0}^{\prime} \times \psi_{0}^{\prime-1}\right)} .
$$

3.2. Quadratic base change. - We recall some properties of the functorial transfers bc : $\Pi^{\prime} \rightarrow \Pi^{\tau}$ given by quadratic base change (cf. [AC89, Ch. 1, §§6, 7]) and ai : $\Pi^{\mathrm{G}_{n}} \rightarrow$ $\Pi^{\prime \mathrm{G}_{2 n}^{\prime}}$ given by automorphic induction (cf. [HH95, Hen 10]).

For any $\pi_{i}^{\prime} \in \Pi^{\mathrm{G}_{n_{i}}^{\prime}}, i=1,2$ we have

$$
\mathrm{L}\left(s, \mathrm{bc}\left(\pi_{1}^{\prime}\right) \times \mathrm{bc}\left(\pi_{2}^{\prime}\right)\right)=\mathrm{L}\left(s, \pi_{1}^{\prime} \times \pi_{2}^{\prime}\right) \mathrm{L}\left(s, \pi_{1}^{\prime} \times \pi_{2}^{\prime} \cdot \eta\right)
$$

and

$$
\varepsilon\left(s, \mathrm{bc}\left(\pi_{1}^{\prime}\right) \times \mathrm{bc}\left(\pi_{2}^{\prime}\right) ; \psi\right)=\lambda_{\psi^{\prime}}^{-n_{1} n_{2}} \varepsilon\left(s, \pi_{1}^{\prime} \times \pi_{2}^{\prime} ; \psi^{\prime}\right) \varepsilon\left(s, \pi_{1}^{\prime} \times \pi_{2}^{\prime} \cdot \eta ; \psi^{\prime}\right),
$$

where $\boldsymbol{\lambda}_{\psi^{\prime}}=\lambda\left(\mathrm{E} / \mathrm{F}, \psi^{\prime}\right)$ is Langlands's constant. Consequently,

$$
\gamma\left(s, \mathrm{bc}\left(\pi_{1}^{\prime}\right) \times \mathrm{bc}\left(\pi_{2}^{\prime}\right) ; \psi\right)=\lambda_{\psi^{\prime}}^{-n_{1} n_{2}} \gamma\left(s, \pi_{1}^{\prime} \times \pi_{2}^{\prime} ; \psi^{\prime}\right) \gamma\left(s, \pi_{1}^{\prime} \times \pi_{2}^{\prime} \cdot \eta ; \psi^{\prime}\right) .
$$

Recall that bc is onto. For any $\pi \in \Pi^{\tau}$, we denote by $\mathcal{B}(\pi)=\mathrm{bc}^{-1}(\{\pi\})$ the fiber of $\pi$ under bc. The set $\mathcal{B}(\pi)$ is finite and we denote its cardinality by $\mathfrak{b}_{\pi}$. For any $\pi^{\prime} \in \Pi^{\prime}$ we have $\mathrm{bc}\left(\pi^{\prime}\right)=\mathrm{bc}\left(\pi^{\prime} \cdot \eta\right)$ and therefore, the group $\left\{1_{\mathrm{F}^{*}}, \eta\right\}$ acts on the set $\mathcal{B}(\pi)$. We denote by $[\mathcal{B}](\pi)$ the set of $\left\{1_{\mathrm{F}^{*}}, \eta\right\}$-orbits in $\mathcal{B}(\pi)$ and by $[\mathfrak{b}]_{\pi}$ the cardinality of $[\mathcal{B}](\pi)$.

In the split case, $\mathrm{bc}\left(\pi^{\prime}\right)=\pi^{\prime} \otimes \pi^{\prime}$ for any $\pi^{\prime} \in \Pi^{\prime}$ and in particular bc is one-toone. From now on, assume that $\mathrm{E} / \mathrm{F}$ is inert.

Let $\delta \in \Pi_{\text {sqr }}^{\tau}$. Then there exists $\delta^{\prime} \in \Pi_{\text {sqr }}^{\prime}$ such that $\delta^{\prime} \not \delta^{\prime} \cdot \eta$ and $\mathcal{B}(\delta)=\left\{\delta^{\prime}, \delta^{\prime} \cdot \eta\right\}$. In particular, $\mathfrak{b}_{\delta}=2$ and $[\mathfrak{b}]_{\delta}=1$. Moreover, in the $p$-adic case $\delta \in \Pi_{\text {cusp }}$ if and only if $\delta^{\prime} \in \Pi_{\text {cusp }}^{\prime}$. To describe the situation for tempered representations, we consider an- 
other example first. Let $\delta \in \Pi_{\text {sqr }} \backslash \Pi_{\text {sqr }}^{\tau}$. Then $\delta \times \delta^{\tau} \in \Pi_{\text {temp }}^{\tau}$ and $\mathcal{B}\left(\delta \times \delta^{\tau}\right)$ is a singleton $\left\{\delta^{\prime}\right\}$, with $\delta^{\prime} \in \Pi_{\mathrm{sqr}}^{\prime}$. This $\delta^{\prime}$ is the automorphic induction of $\delta$, denoted ai $(\delta)$. In particular, ai $(\delta) \simeq \operatorname{ai}(\delta) \cdot \eta=\operatorname{ai}\left(\delta^{\tau}\right)$ and we have $\mathfrak{b}_{\delta \times \delta^{\tau}}=[\mathfrak{b}]_{\delta \times \delta^{\tau}}=1$. Again, in the $p$ adic case $\delta \in \Pi_{\text {cusp }}$ if and only if ai $(\delta) \in \Pi_{\text {cusp }}^{\prime}$. In general, any $\pi \in \Pi_{\text {temp }}$ is of the form $\delta_{1} \times \cdots \times \delta_{t}$ with $\delta_{1}, \ldots, \delta_{t} \in \Pi_{\text {usqr }}$ which are uniquely determined up to a permutation. Thus any $\pi \in \Pi_{\text {temp }}^{\tau}$ can be written as $\pi=\delta \times \sigma \times \sigma^{\tau}$ where $\delta=\delta_{1} \times \cdots \times \delta_{r} \in \Pi_{\text {temp }}^{\mathrm{G}_{l}, \tau}$, $\sigma=\sigma_{1} \times \cdots \times \sigma_{s} \in \Pi_{\text {temp }}^{\mathrm{G}_{k}}$ with $\delta_{1}, \ldots, \delta_{r} \in \Pi_{\text {usqr }}^{\tau}, \sigma_{1}, \ldots, \sigma_{s} \in \Pi_{\text {usqr }} \backslash \Pi_{\text {usqr }}^{\tau}$. The $\delta_{i}$ 's and the pairs $\left\{\sigma_{j}, \sigma_{j}^{\tau}\right\}, j=1, \ldots, s$ are uniquely determined by $\pi$ up to permutation. We call the integer $0 \leq k \leq n / 2$ the $\tau$-Witt index of $\pi$ and denote it by $\tilde{\mathfrak{w}}(\pi)$. Note that in the Archimedean case, $r+2 s=n$, i.e., the $\delta_{i}$ 's and $\sigma_{j}$ 's are characters of $\mathbf{C}^{*}$, with $\delta_{i}$ unramified, and $\tilde{\mathfrak{w}}(\pi)=s$.

In analogy with quadratic forms, we say that $\pi$ is totally $\tau$-isotropic (resp., $\tau$ anisotropic) if $\tilde{\mathfrak{w}}(\pi)=n / 2$ (resp., $\tilde{\mathfrak{w}}(\pi)=0)$. We have $\pi^{\prime} \in \mathcal{B}(\pi)$ if and only if $\pi^{\prime} \simeq$ $\delta^{\prime} \times \operatorname{ai}(\sigma)$ where $\operatorname{ai}(\sigma)=\operatorname{ai}\left(\sigma_{1}\right) \times \cdots \times \operatorname{ai}\left(\sigma_{s}\right)$ and $\delta^{\prime} \in \mathcal{B}(\delta)$, i.e. $\delta^{\prime}=\delta_{1}^{\prime} \times \cdots \times \delta_{r}^{\prime}$ where $\delta_{i}^{\prime} \in \mathcal{B}\left(\delta_{i}\right), i=1, \ldots, r$. Writing

$$
\delta=\overbrace{\Delta_{1} \times \cdots \times \Delta_{1}}^{k_{1}} \times \cdots \times \overbrace{\Delta_{m} \times \cdots \times \Delta_{m}}^{k_{m}}
$$

with $\Delta_{i} \in \Pi_{\text {usqr }}^{\tau}$ distinct and $k_{1}+\cdots+k_{m}=r$ and fixing a choice $\Delta_{i}^{\prime} \in \mathcal{B}\left(\Delta_{i}\right)$ for each $i$, any $\delta^{\prime} \in \mathcal{B}(\delta)$ can be written uniquely as

$$
\begin{aligned}
& \overbrace{\Delta_{1}^{\prime} \times \cdots \times \Delta_{1}^{\prime}}^{j_{1}} \times \overbrace{\Delta_{1}^{\prime} \cdot \eta \times \cdots \times \Delta_{1}^{\prime} \cdot \eta}^{k_{1}-j_{1}} \times \cdots \times \overbrace{\Delta_{m}^{\prime} \times \cdots \times \Delta_{m}^{\prime}}^{j_{m}} \\
& \times \overbrace{\Delta_{m}^{\prime} \cdot \eta \times \cdots \times \Delta_{m}^{\prime} \cdot \eta}^{k_{m}-j_{m}}
\end{aligned}
$$

where $0 \leq j_{i} \leq k_{i}, i=1, \ldots, m$. In particular $\mathfrak{b}_{\pi}=\left(k_{1}+1\right) \ldots\left(k_{m}+1\right) \leq 2^{r}$. Thus, $\mathfrak{b}_{\pi}=2^{r}$ if and only if $m=r$, i.e., if and only if $\delta_{1}, \ldots, \delta_{r}$ are distinct, and $\mathfrak{b}_{\pi}=1$ if and only if $\tilde{\mathfrak{w}}(\pi)=n / 2$ (i.e. $r=0$ ).

For any $\pi^{\prime} \in \Pi_{\text {temp }}^{\prime}$ we define $\tilde{\mathfrak{w}}^{\prime}\left(\pi^{\prime}\right)$ (which we call the $\eta$-Witt index of $\pi^{\prime}$ ) to be the maximal integer $0 \leq k \leq n / 2$ such that $\pi^{\prime}=\operatorname{ai}(\delta) \times \sigma^{\prime}$ where $\delta \in \Pi_{\text {temp }}^{\mathrm{G}_{k}}$. In particular, $\tilde{\mathfrak{w}}^{\prime}\left(\pi^{\prime}\right)=n / 2$ (and we say that $\pi^{\prime}$ is totally $\eta$-isotropic) if and only if $\pi^{\prime} \simeq \pi^{\prime} \cdot \eta$ (in which case $n$ is even). In this case, $\pi^{\prime}=\operatorname{ai}(\delta)$ for $\delta \in \Pi_{\text {temp }}^{\mathrm{G}_{n / 2}}$ (not necessarily unique) and bc $\left(\pi^{\prime}\right)=$ $\delta \times \delta^{\tau}$. Conversely, if $\pi=\delta \times \delta^{\tau}$ for some $\delta \in \Pi_{\text {temp }}^{\mathrm{G}_{n / 2}}$ then ai $(\delta)$ depends only on $\pi$ and it is the unique totally $\eta$-isotropic element $\pi^{\prime} \in \mathcal{B}(\pi)$.

Note that for any $\pi \in \Pi_{\text {temp }}^{\tau}$ we have

$$
\tilde{\mathfrak{w}}(\pi)=\min _{\pi^{\prime} \in \mathcal{B}(\pi)} \tilde{\mathfrak{w}}^{\prime}\left(\pi^{\prime}\right)
$$

Now let $\pi \in \Pi^{\tau}$ be of the form $\operatorname{LQ}(\sigma, \lambda)$ where $\sigma \in \Pi_{\text {temp }}^{\mathrm{M}}$ and $\lambda \in\left(\mathfrak{a}_{\mathrm{M}}^{*}\right)_{+}$. The uniqueness part of the Langlands classification implies that $\sigma \in \Pi^{\mathrm{M}, \tau}$. We have 
$\mathcal{B}(\pi)=\left\{\operatorname{LQ}\left(\sigma^{\prime}, \lambda\right): \sigma^{\prime} \in \mathcal{B}(\sigma)\right\}$ and in particular $\mathfrak{b}_{\pi}=\mathfrak{b}_{\sigma}=\prod_{i=1}^{t} \mathfrak{b}_{\sigma_{i}}$ where $\sigma=$ $\sigma_{1} \otimes \cdots \otimes \sigma_{t}$ and $\sigma_{i} \in \Pi_{\text {temp }}^{\mathrm{G}_{n_{i}}, \tau}, i=1, \ldots, t$. Set $\tilde{\mathfrak{w}}(\pi):=\tilde{\mathfrak{w}}(\sigma)=\sum_{i=1}^{t} \tilde{\mathfrak{w}}\left(\sigma_{i}\right)$ - the $\tau$-Witt index of $\pi$. As before, we say that $\pi$ is $\tau$-anisotropic (resp. totally $\tau$-isotropic) if $\tilde{\mathfrak{w}}(\pi)=0$ (resp. $\tilde{\mathfrak{w}}(\pi)=n / 2$ ). Once again, $\pi$ is totally $\tau$-isotropic if and only if $\mathfrak{b}_{\pi}=1$. We denote by $\Pi^{\tau, \mathrm{ti}}\left(\right.$ resp. $\left.\Pi^{\tau, \text { an }}\right)$ the set of totally $\tau$-isotropic (resp. $\tau$-anisotropic) representations.

Analogously, we write

$$
\tilde{\mathfrak{w}}^{\prime}\left(\operatorname{LQ}\left(\sigma^{\prime}, \lambda\right)\right):=\tilde{\mathfrak{w}}^{\prime}\left(\sigma^{\prime}\right)=\sum_{i=1}^{t} \tilde{\mathfrak{w}}^{\prime}\left(\sigma_{i}^{\prime}\right) \leq n / 2
$$

and define totally $\eta$-isotropic and $\eta$-anisotropic in a similar vein. Thus, $\pi^{\prime}$ is totally $\eta$ isotropic, i.e., $\tilde{\mathfrak{w}}^{\prime}\left(\pi^{\prime}\right)=n / 2$, if and only if $\pi^{\prime} \simeq \pi^{\prime} \cdot \eta$. Note that there exists a totally $\eta$-isotropic $\pi^{\prime} \in \mathcal{B}(\pi)$ if and only if the same property holds for $\sigma_{1}, \ldots, \sigma_{t}$ and in this case such $\pi^{\prime}$ is unique, indeed it is $\operatorname{LQ}\left(\sigma^{\prime}, \lambda\right)$ where $\sigma^{\prime}=\sigma_{1}^{\prime} \otimes \cdots \otimes \sigma_{r}^{\prime}$ and $\sigma_{i}^{\prime} \in \mathcal{B}\left(\sigma_{i}\right)$ is the unique element such that $\sigma_{i}^{\prime} \simeq \sigma_{i}^{\prime} \cdot \eta$.

\section{Lemma 3.2.}

(1) Suppose that $\pi=\mathrm{I}(\sigma, \lambda) \in \Pi_{\text {gen }}^{\tau}$. Then for any $\sigma^{\prime} \in \mathcal{B}(\sigma), \mathrm{I}\left(\sigma^{\prime}, \lambda\right)$ is irreducible. Hence, $\mathcal{B}(\pi)=\left\{\mathrm{I}\left(\sigma^{\prime}, \lambda\right): \sigma^{\prime} \in \mathcal{B}(\sigma)\right\}$.

(2) Any $\pi \in \Pi_{\text {gen }}^{\tau}$ can be written as $\pi=\delta \times \sigma \times \sigma^{\tau}$ where $\delta \in \Pi_{\text {gen }}^{\tau}$ is $\tau$-anisotropic, $\sigma \in \Pi_{\text {gen }}^{\mathrm{G}_{k}}$ and $k=\tilde{\mathfrak{w}}(\pi)$. Moreover, $\delta$ and $\mathrm{ai}(\sigma)$ (but not necessarily $\sigma$ itself) are uniquely determined by $\pi$.

(3) Similarly any $\pi^{\prime} \in \Pi_{\text {gen }}^{\prime}$ can be written as $\pi^{\prime}=\operatorname{ai}(\sigma) \times \delta^{\prime}$ where $\sigma \in \Pi_{\text {gen }}^{\mathrm{G}_{k}}, k=$ $\tilde{\mathfrak{w}}^{\prime}\left(\pi^{\prime}\right)$ and $\delta^{\prime}$ is $\eta$-anisotropic. Moreover, $\delta^{\prime}$ and $\mathrm{ai}(\sigma)$ (but not necessarily $\sigma$ ) are uniquely determined by $\pi^{\prime}$.

Proof. - The first part follows from $(\mathbf{1 . 1 1})$ and $(\mathbf{3 . 7})$. The remaining assertions are straightforward.

We observe that bc does not take $\Pi_{\text {gen }}^{\prime}$ to $\Pi_{\text {gen }}$. For instance, bc $\left(|\cdot|_{\mathrm{F}} \times \eta\right)=|\operatorname{det}|_{\mathrm{E}}^{\frac{1}{2}}$. We denote by $\Pi_{\mathrm{bc} \text {-gen }}^{\prime}$ the set of $\pi^{\prime} \in \Pi_{\text {gen }}^{\prime}$ such that $\operatorname{bc}\left(\pi^{\prime}\right) \in \Pi_{\text {gen }}$. More explicitly, $\Pi_{\mathrm{bc}-\mathrm{gen}}^{\prime}$ consists of the irreducible generic representations of the form $\sigma_{1}^{\prime} \times \cdots \times \sigma_{k}^{\prime}$ where $\sigma_{1}^{\prime}, \ldots, \sigma_{k}^{\prime} \in \Pi_{\text {sqr }}^{\prime}$ are such that $\sigma_{i}^{\prime} \eta \times \sigma_{j}^{\prime}$ is irreducible for all $i \neq j$ or equivalently such that $\operatorname{bc}\left(\sigma_{i}^{\prime}\right) \times \operatorname{bc}\left(\sigma_{j}^{\prime}\right)$ is irreducible for all $i \neq j$.

The following Lemmas are straightforward.

Lemma 3.3. - We have $\Pi_{\mathrm{unr}} \subseteq \Pi^{\tau, \mathrm{an}}$. In the Archimedean case, $\Pi_{\mathrm{unr}}=\Pi^{\tau \text {,an }}$.

Lemma 3.4. - Suppose that $\mathrm{F}$ is p-adic and let $\pi \in \Pi^{\tau}$. Then $\pi \in \Pi^{\tau, \mathrm{ti}}$ (resp. $\pi \in \Pi^{\tau, \text { an }}$ ) if and only if $\operatorname{supp}_{c}(\pi) \cap \Pi^{\tau}=\emptyset\left(\right.$ resp. $\left.\operatorname{supp}_{c}(\pi) \subseteq \Pi^{\tau}\right)$. It follows that 
(1) Any $\pi_{1} \in \Pi^{\tau, \mathrm{ti}}$ and $\pi_{2} \in \Pi^{\tau, \text { an }}$ are disjoint.

(2) If $\pi \in \Pi^{\tau, \text { an }}$ then all pure components of $\pi$ are in $\Pi^{\tau, \text { an }}$.

(3) $\pi \in \Pi^{\tau, \mathrm{ti}}$ if and only if we can write (non-uniquely) $\pi=\delta \times \delta^{\tau}$ where $\delta \in \Pi^{\mathrm{G}_{n / 2}}$ and $\delta^{\tau}$ are disjoint.

(4) Any $\pi \in \Pi^{\tau}$ can be written uniquely as $\pi=\pi_{1} \times \pi_{2}$ where $\pi_{1} \in \Pi^{\tau, \mathrm{ti}}$ and $\pi_{2} \in \Pi^{\tau, \text { an }}$. Moreover, we have $\mathfrak{b}_{\pi}=\mathfrak{b}_{\pi_{2}}$. we have

Finally, we mention the following property. For $\sigma^{\prime} \in \Pi^{\mathrm{G}_{n}^{\prime}}, \sigma=\operatorname{bc}\left(\sigma^{\prime}\right)$ and $\varrho \in \Pi^{\mathrm{G}_{m}}$

$$
\begin{aligned}
& \mathrm{L}\left(s, \sigma^{\prime} \times \operatorname{ai}(\varrho)\right)=\mathrm{L}(s, \sigma \times \varrho), \\
& \varepsilon\left(s, \sigma^{\prime} \times \operatorname{ai}(\varrho), \psi^{\prime}\right)=\lambda_{\psi^{\prime}}^{m n} \varepsilon(s, \sigma \times \varrho, \psi) .
\end{aligned}
$$

Hence,

$$
\gamma\left(s, \sigma^{\prime} \times \operatorname{ai}(\varrho), \psi^{\prime}\right)=\lambda_{\psi^{\prime}}^{m n} \gamma(s, \sigma \times \varrho, \psi) .
$$

Note also that

$$
\omega_{\mathrm{ai}(\varrho)}=\left.\omega_{\varrho}\right|_{\mathrm{F} *} \eta^{m}
$$

3.3. The Bessel identities - $\mathcal{B I}$. - Our goal is to define for any $\pi \in \Pi_{\text {gen }}^{\tau}$ and $\pi^{\prime} \in \mathcal{B}(\pi)$ a certain element $\alpha^{\pi^{\prime}} \in \mathcal{E}_{\mathrm{G}}\left(\mathrm{X}, \mathcal{W}(\pi)^{*}\right)$, i.e., a compatible family of local unitary periods on the Whittaker model of $\pi$. These local periods will turn out to be the local components of global unitary periods. The definition is given implicitly via an identity of Bessel distributions which will only be established in full generality in Section 12. Provisionally, we define a subset $\mathcal{B I}_{x}$ of $\Pi_{\mathrm{bc} \text {-gen }}^{\prime}$ for any $x \in \mathrm{X}$ as follows. Suppose that $\pi^{\prime} \in \Pi_{\mathrm{bc}-\text { gen }}^{\prime}$ and let $\pi=\mathrm{bc}\left(\pi^{\prime}\right)$. Then by definition, $\pi^{\prime} \in \mathcal{B I}_{x}$ if there exists a linear form $\alpha_{x}^{\pi^{\prime}} \in \mathcal{W}(\pi)^{*}$ (possibly zero, but necessarily unique by Lemma 2.2 and smooth transfer) satisfying

$$
\mathfrak{B}_{\mathfrak{W}(\pi)}^{\alpha_{\pi}^{\pi^{\prime}}, \delta_{e}^{\pi^{\vee}}}(f)=\mathbf{B}_{\pi^{\prime}}\left(f^{\prime}\right)
$$

for any $f \in \mathcal{S}(\mathrm{G})$ and $f^{\prime} \in \mathcal{S}\left(\mathrm{G}^{\prime}\right)$ such that $f \stackrel{x}{\longleftrightarrow} f^{\prime}$. For convenience, we set $\alpha_{x}^{\pi^{\prime}}=0$ in the (a posteriori empty) case $\pi^{\prime} \notin \mathcal{B \mathcal { I } _ { x }}$. We also set

$$
\mathcal{B I}=\mathcal{B I}^{\mathrm{G}}=\bigcap_{x \in \mathrm{X}} \mathcal{B I}_{x}
$$

In the split case, it follows from [Jac01, Lemma 2] that every unitarizable $\pi^{\prime} \in \Pi_{\mathrm{bc} \text {-gen }}^{\prime}=$ $\Pi_{\text {gen }}^{\prime}$ belongs to $\mathcal{B I}$. The argument easily extends to any $\pi^{\prime} \in \Pi_{\text {gen }}^{\prime}$ - cf. Corollary 7.2 below. Eventually, we will show that $\mathcal{B I}=\Pi_{\mathrm{bc} \text {-gen }}^{\prime}$ in the inert case as well-cf. Theorem 12.4 below. 
Suppose that $\pi^{\prime} \in \mathcal{B} \mathcal{I}_{x}$ for some $x \in \mathrm{X}$ and let $\pi=\mathrm{bc}\left(\pi^{\prime}\right)$. Then it follows from (3.3) and (2.1) that for any $g \in \mathrm{G}$ we have $\pi^{\prime} \in \mathcal{B I}_{x \bullet g}$ and

$$
\alpha_{x \bullet g}^{\pi^{\prime}}=\alpha_{x}^{\pi^{\prime}} \circ \mathcal{W}(g, \pi)
$$

In particular, $\alpha_{x}^{\pi^{\prime}} \in \operatorname{Hom}_{\mathrm{G}^{x}}(\mathcal{W}(\pi), \mathbf{C})$. Recall that by $(\mathbf{2 . 1})$, for any $\ell \in \operatorname{Hom}_{\mathrm{G}^{x}}(\mathcal{W}(\pi), \mathbf{C})$, $\mathfrak{B}_{\mathfrak{W}(\pi)}^{\ell, \delta_{e}^{\pi^{\vee}}}(f)$ is right $\left(\mathrm{U}_{0}, \psi_{0}^{-1}\right)$-equivariant and left $\mathrm{G}^{x}$-invariant, and therefore, at least in the $p$-adic case, it depends only on the orbital integrals of $f$.

Suppose that $\pi^{\prime} \in \mathcal{B I}$. It follows that $\alpha^{\pi^{\prime}} \in \mathcal{E}\left(\mathrm{X}, \mathcal{W}(\pi)^{*}\right)$ (so that $\alpha_{x}^{\pi^{\prime}}$ is $\mathrm{G}^{x}$ invariant for all $x \in \mathrm{X})$. Therefore, by $(\mathbf{2 . 7})$ we have $\tilde{\mathfrak{B}}_{\mathfrak{W}(\pi)}^{\alpha^{\pi^{\prime}}, \delta_{e} \vee} \in \mathcal{S}^{*}(\mathrm{X})^{\left(\mathrm{U}_{0}, \psi_{0}^{-1}\right)}$ and by definition we have

$$
\tilde{\mathfrak{B}}_{\mathfrak{W}(\pi)}^{\alpha^{\pi^{\prime}}, \delta_{e}^{\pi^{\vee}}} \longleftrightarrow \mathbf{B}_{\pi^{\prime}}
$$

For a character $\chi$ of $\mathrm{E}^{*}$ and $\pi \in \Pi_{\text {gen }}$, recall the equivalence $\mathrm{A}_{\chi}^{\pi}: \mathcal{W}(\pi) \cdot \chi \rightarrow$ $\mathcal{W}(\pi \cdot \chi)$ defined by $\mathrm{A}_{\chi}^{\pi}(\mathrm{W})=\mathrm{W}_{\chi}, \mathrm{W} \in \mathcal{W}(\pi)$.

Lemma 3.5. - Suppose that $\pi^{\prime} \in \mathcal{B I}_{x}$ for some $x \in \mathrm{X}$ and let $\pi=\operatorname{bc}\left(\pi^{\prime}\right)$. Then for any character $\chi^{\prime}$ of $\mathrm{F}^{*}$ we have $\pi^{\prime} \cdot \chi^{\prime} \in \mathcal{B I}_{x}$ and

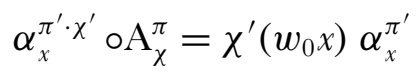

where $\chi=\chi^{\prime} \circ \mathrm{Nm}$. In particular,

$$
\alpha_{x}^{\pi^{\prime} \cdot \eta}=\eta\left(w_{0} x\right) \alpha_{x}^{\pi^{\prime}}
$$

Proof. - Note that for $f \stackrel{x}{\longleftrightarrow} f^{\prime}$ we also have $\chi^{\prime}(x) f_{\chi} \stackrel{x}{\longleftrightarrow} f_{\chi^{\prime}}^{\prime}$ and therefore

$$
\chi^{\prime}(x) \mathfrak{B}_{\mathfrak{W}(\pi)}^{\alpha_{x}^{\pi^{\prime}}, \delta_{e}^{\pi^{\vee}}}\left(f_{\chi}\right)=\mathbf{B}_{\pi^{\prime}}\left(f_{\chi^{\prime}}^{\prime}\right) .
$$

Combined with $(\mathbf{2 . 1 5})$ this gives

$$
\mathbf{B}_{\pi^{\prime} \cdot \chi^{\prime}}\left(f^{\prime}\right)=\chi^{\prime}\left(w_{0} x\right) \mathfrak{B}_{\mathfrak{W}(\pi)}^{\alpha_{x}^{\pi^{\prime}}, \delta_{e}^{\pi^{\vee}}}\left(f_{\chi}\right) .
$$

On the other hand, as in the proof of $(\mathbf{2 . 1 5})$ we have

$$
\mathfrak{B}_{\mathfrak{W}(\pi)}^{\alpha_{x}^{\pi^{\prime}}, \delta_{e}^{\pi^{\vee}}}\left(f_{\chi}\right)=\mathfrak{B}_{\mathfrak{W}(\pi) \cdot \chi}^{\alpha_{x}^{\pi^{\prime}}, \delta_{e}^{\pi^{\vee}}}(f)
$$

by $(\mathbf{2 . 3})$, and then applying $(\mathbf{2 . 4})$ to $\left(\mathrm{A}_{\chi}^{\pi}, \mathrm{A}_{\chi^{-1}}^{\pi^{\vee}}\right)$,

$$
\mathfrak{B}_{\mathfrak{W}(\pi) \cdot \chi}^{\alpha_{x}^{\pi^{\prime}}, \delta_{e}^{\pi^{\vee}}}=\mathfrak{B}_{\mathfrak{W}(\pi \cdot \chi)}^{\ell, \delta_{e}^{\pi^{\vee}} \cdot \chi^{-1}}
$$

where $\ell \circ \mathrm{A}_{\chi}^{\pi}=\alpha_{x}^{\pi^{\prime}}$. Hence (3.12) follows. 
For induction purpose, it will be useful to define more generally subsets $\mathcal{B I}_{\mathrm{M}, x}$ of $\Pi_{\mathrm{bc}-\text { gen }}^{\mathrm{M}^{\prime}}$ for any $\mathrm{M}$ and $x \in \mathrm{X}$ as follows. We write $\sigma^{\prime} \in \mathcal{B I}_{\mathrm{M}, x}$ if there exists a meromorphic family $\lambda \mapsto \alpha_{\mathrm{M}, x}\left(\sigma^{\prime}, \lambda\right) \in \mathrm{I}(\mathcal{W}(\sigma), \lambda)^{*}$ on $\mathfrak{a}_{\mathrm{M}, \mathbf{G}}^{*}$ such that

$$
\mathfrak{B}_{\mathrm{I}_{\mathrm{M}}^{\mathrm{G}}(\mathfrak{W}(\sigma), \lambda)}^{\alpha_{\mathrm{W}}\left(\sigma^{\prime}, \lambda\right), \mathbf{W}_{e}\left(\sigma^{\vee},-\lambda\right)}(f)=\mathbf{B}\left(f^{\prime}: \sigma^{\prime}, \lambda\right)
$$

as meromorphic functions whenever $f \stackrel{x}{\longleftrightarrow} f^{\prime}$. Here, as usual, $\sigma=\operatorname{bc}\left(\sigma^{\prime}\right)$. We further set $\mathcal{B I}_{\mathrm{M}}:=\bigcap_{x \in \mathrm{X}} \mathcal{B I}_{\mathrm{M}, x}$.

Once again, it follows from $(3.3)$ and $(2.1)$ that if $\sigma^{\prime} \in \mathcal{B I}_{\mathrm{M}, x}$ then $\sigma^{\prime} \in \mathcal{B I}_{\mathrm{M}, x \bullet g}$ for all $g \in \mathrm{G}$ and

$$
\alpha_{\mathrm{M}, x \circ g}\left(\sigma^{\prime}, \lambda\right)=\alpha_{\mathrm{M}, x}\left(\sigma^{\prime}, \lambda\right) \circ \mathrm{I}(g, \mathcal{W}(\sigma), \lambda)
$$

where $\sigma=\operatorname{bc}\left(\sigma^{\prime}\right)$.

Lemma 3.6. - Let $\pi^{\prime}=\mathrm{I}\left(\sigma^{\prime}, \lambda\right) \in \Pi_{\mathrm{bc}-\text { gen }}^{\prime}$ and suppose that $\sigma^{\prime} \in \mathcal{B I}_{\mathrm{M}, x}$. Then $\alpha_{\mathrm{M}, x}\left(\sigma^{\prime}, \cdot\right)$ is holomorphic at $\lambda, \pi^{\prime} \in \mathcal{B I}_{x}$ and $\alpha_{x}^{\pi^{\prime}} \circ \mathbf{W}(\sigma, \lambda)=\alpha_{\mathrm{M}, x}\left(\sigma^{\prime}, \lambda\right)$.

Proof. - The holomorphicity follows from Lemma 2.3 and smooth transfer. The rest of the Lemma follows from (2.4) applied to the equivalence $\Omega(\sigma, \lambda)$ defined in $(\mathbf{2 . 1 3})$ and the identity $(\mathbf{2 . 1 4})$.

Lemma 3.7. - For $\sigma^{\prime} \in \mathcal{B I}_{\mathrm{M}}$ and $\mu \in \mathfrak{a}_{\mathrm{M}, \mathbf{C}}^{*}$ we have $\sigma^{\prime}[\mu] \in \mathcal{B I}_{\mathrm{M}}$ and

$$
\alpha_{\mathrm{M}}\left(\sigma^{\prime}[\mu], \lambda\right)=\alpha_{\mathrm{M}}\left(\sigma^{\prime}, \mu+\lambda\right) \circ \mathcal{A}(\sigma, \mu), \quad \lambda \in \mathfrak{a}_{\mathrm{M}, \mathbf{G}}^{*}
$$

where $\sigma=\operatorname{bc}\left(\sigma^{\prime}\right)$ and

$$
\mathcal{A}(\sigma, \mu)=\mathrm{I}\left(\mathrm{A}_{e^{-\langle\mu, \mathrm{H}(\cdot)\rangle}}^{\sigma[\mu]}\right): \mathrm{I}(\mathcal{W}(\sigma[\mu]), \lambda) \rightarrow \mathrm{I}(\mathcal{W}(\sigma), \mu+\lambda)
$$

i.e., $\mathcal{A}(\sigma, \mu) \varphi(g)=e^{-\langle\mu, \mathrm{H}(\cdot)\rangle} \varphi(g), g \in \mathrm{G}$.

Proof. - It is easy to see that $\left(\mathcal{A}(\sigma, \mu), \mathcal{A}\left(\sigma^{\vee},-\mu\right)\right): \mathrm{I}(\mathfrak{W}(\sigma[\mu]), \lambda) \rightarrow \mathrm{I}(\mathfrak{W}(\sigma)$, $\mu+\lambda)$ is an equivalence of dual couples and that $\mathbf{W}_{e}\left(\sigma^{\vee},-\mu-\lambda\right) \circ \mathcal{A}\left(\sigma^{\vee},-\mu\right)=$ $\mathbf{W}_{e}\left(\sigma^{\vee}[-\mu],-\lambda\right)$. Applying $(\mathbf{2 . 9})$ we get that

$$
\tilde{\mathfrak{B}}_{\mathrm{I}_{\mathrm{M}}^{\mathrm{G}}(\mathfrak{W}(\sigma[\mu]), \lambda)}^{\alpha_{\mathrm{M}}\left(\sigma^{\prime}, \mu+\lambda\right) \mathcal{A}(\sigma, \mu), \mathbf{W}_{e}\left(\sigma^{\vee}[-\mu],-\lambda\right)}=\tilde{\mathfrak{B}}_{\mathrm{I}_{\mathrm{M}}^{\mathrm{G}}(\mathfrak{W}(\sigma), \mu+\lambda)}^{\alpha_{\mathrm{M}}\left(\sigma^{\prime}, \mu+\lambda\right), \mathbf{W}_{e}\left(\sigma^{\vee},-\mu-\lambda\right)} .
$$

It follows from (2.14) that $\mathbf{B}\left(\sigma^{\prime}[\mu], \lambda\right)=\mathbf{B}\left(\sigma^{\prime}, \mu+\lambda\right)$. Taking $(\mathbf{2 . 1 1})$ into account, the lemma follows.

Assume that $\mathrm{E} / \mathrm{F}$ is inert. Let

$$
\mathrm{X}^{+}=\mathrm{X}\left[w_{0}\right] \text { and } \mathrm{X}^{-}=\mathrm{X} \backslash \mathrm{X}^{+} .
$$

From the support property of the normalized Bessel distributions (Lemma 2.6) we get the following (partial) vanishing criterion. 
Lemma 3.8. - Assume that $\pi^{\prime} \in \Pi_{\mathrm{bc}-\mathrm{gen}}^{\prime}$ and $x \in \mathrm{X}$. Then

(1) If $x \in \mathrm{X}^{-}$and $\pi^{\prime} \simeq \pi^{\prime} \cdot \eta$ then $\pi^{\prime} \in \mathcal{B \mathcal { I } _ { x }}$ and $\alpha_{x}^{\pi^{\prime}} \equiv 0$.

(2) In the non-Archimedean case, if $\pi^{\prime} \in \mathcal{B I}_{x}$ then $\alpha_{x}^{\pi^{\prime}} \equiv 0$ if and only if $\mathrm{G}^{x}$ is non-quasi-split and $\pi^{\prime} \simeq \pi^{\prime} \cdot \eta$.

Proof. - If $\pi^{\prime} \simeq \pi^{\prime} \cdot \eta$ then by Lemma 2.6, $\mathbf{B}_{\pi^{\prime}}$ vanishes on $\mathcal{S}\left(\mathrm{G}^{\prime-}\right)$. It follows from (3.4) that $\pi^{\prime} \in \mathcal{B} \mathcal{I}_{x}$ and $\alpha_{x}^{\pi^{\prime}} \equiv 0$ for $x \in \mathrm{X}^{-}$.

Conversely, in the non-Archimedean case, (in which $\mathrm{X}[x]$ is a single G-orbit) for any $f^{\prime} \in \mathcal{S}\left(\mathrm{G}^{\prime}[x]\right)$ we can find $f \in \mathcal{S}(\mathrm{G})$ such that $f \stackrel{x}{\longleftrightarrow} f^{\prime}$. Thus, if $\pi^{\prime} \in \mathcal{B I}$ and $\alpha_{x}^{\pi^{\prime}} \equiv 0$ then $\mathbf{B}_{\pi^{\prime}}$ vanishes on $\mathcal{S}\left(\mathrm{G}^{\prime}[x]\right)$. Applying Lemma 2.6 once again, we conclude that $x \in \mathrm{X}^{-}$and $\pi^{\prime} \simeq \pi^{\prime} \cdot \eta$. In particular, $n$ is even and therefore $\mathrm{G}^{x}$ is non-quasi-split.

In the $p$-adic case, once we show that $\Pi_{\mathrm{bc}-\text { gen }}^{\prime}=\mathcal{B I}$, Lemma 3.8 can be restated as

$$
\alpha_{x}^{\pi^{\prime}} \not \equiv 0 \quad \text { if and only if } \quad \tilde{\mathfrak{w}}^{\prime}\left(\pi^{\prime}\right) \leq \mathfrak{w}(x)
$$

where we recall that $\tilde{\mathfrak{w}}^{\prime}\left(\pi^{\prime}\right)$ is defined in Section 3.2 and $\mathfrak{w}(x)$ is the Witt index of $x$. As we shall see later, this criterion holds in the Archimedean case as well.

We conclude this section with a computation for unramified data.

Lemma 3.9. - Assume that $\mathrm{F}$ is non-Archimedean of odd residual characteristic, $\mathrm{E} / \mathrm{F}$ is unramified and $\psi^{\prime}$ has conductor $\mathcal{O}_{\mathrm{F}}$. Let $\pi=\mathrm{bc}\left(\pi^{\prime}\right) \in \Pi_{\text {gen,unr }}$ and $\mathrm{W}_{0}^{\pi} \in \mathcal{W}(\pi)$ the spherical Whittaker function normalized by $\mathrm{W}_{0}^{\pi}(e)=1$. Let $x \in \mathrm{X} \cap \mathrm{K}$ and suppose that $\pi^{\prime} \in \mathcal{B} \mathcal{I}_{x}$. Then

$$
\alpha_{x}^{\pi^{\prime}}\left(\mathrm{W}_{0}^{\pi}\right)=\frac{\mathrm{L}\left(1, \pi \times \pi^{\vee}\right)}{\mathrm{L}\left(1, \pi^{\prime} \times \pi^{\prime \vee}\right)}=\mathrm{L}\left(1, \pi^{\prime} \times \pi^{\prime \vee} \cdot \eta\right) .
$$

Proof. - By (2.5) and (A.4) we have

$$
\mathfrak{B}_{\mathfrak{W}(\pi)}^{\alpha_{x}^{\pi^{\prime}}, \delta_{e}^{\pi^{\vee}}}\left(\mathbf{1}_{\mathrm{K}}\right)=\frac{\alpha_{x}^{\pi^{\prime}}\left(\mathrm{W}_{0}^{\pi}\right)}{\left[\mathrm{W}_{0}^{\pi}, \mathrm{W}_{0}^{\pi^{\vee}}\right]_{\pi}}=\frac{\alpha_{x}^{\pi^{\prime}}\left(\mathrm{W}_{0}^{\pi}\right)}{\mathrm{L}\left(1, \pi \times \pi^{\vee}\right)} .
$$

On the other hand, by $(\mathbf{3 . 6})$, the defining property of $\alpha_{x}^{\pi^{\prime}}$, and $(\mathbf{2 . 1 7})$ we have

$$
\mathfrak{B}_{\mathfrak{W}(\pi)}^{\alpha_{x}^{\pi^{\prime}}, \delta_{e}^{\pi^{\vee}}}\left(\mathbf{1}_{\mathrm{K}}\right)=\mathbf{B}_{\pi^{\prime}}\left(\mathbf{1}_{\mathrm{K}^{\prime}}\right)=\mathrm{L}\left(1, \pi^{\prime} \times \pi^{\prime \vee}\right)^{-1}
$$

The Lemma follows.

\section{Open local periods}

The local periods that we are going to study in this section were introduced in [LR00] for principal series representations. They were further analyzed in [Off07]. Here we introduce analogous objects for more general induced representations of finite length. 
Fix a standard parabolic $\mathbf{P}=\mathbf{M U}$. Note that $\mathbf{P}$ has an open orbit $\mathbf{X}^{\circ}=\mathbf{X} \cap{ }^{\boldsymbol{t}} \mathbf{U M U}$ on $\mathbf{X}$. While $\mathrm{X}^{\circ}$ is not a single $\mathrm{P}$ orbit in general, any $\mathrm{P}$ orbit in $\mathrm{X}^{\circ}$ intersects $\mathrm{X}^{\mathrm{M}}=\mathrm{X} \cap \mathrm{M}$ in a single $\mathrm{M}$-orbit. Therefore, $\mathrm{X}^{\circ} / \mathrm{P}$ is in bijection with the finite set $\mathrm{X}^{\mathrm{M}} / \mathrm{M}$. The $\mathrm{P}$-orbits in $\mathrm{X}^{\circ}$ are the open orbits of $\mathrm{P}$ in $\mathrm{X}$ (in the usual topology).

Let $v_{\mathrm{M}}=v_{\mathrm{M}}^{\mathrm{G}}$ be either the character of $\mathrm{M}^{\prime}$ or the function on $\mathrm{X}^{\mathrm{M}}$ defined by

$$
v_{\mathrm{M}}(g)=\prod_{i=1}^{t} \eta^{i-1}\left(\operatorname{det} g_{i}\right), \quad g=\operatorname{diag}\left(g_{1}, \ldots, g_{t}\right)
$$

and set further $v_{0}=v_{\mathrm{M}_{0}}$.

Let $\sigma \in \mathcal{R}(\mathrm{M})$ and let $\alpha \in \mathcal{E}_{\mathrm{M}}\left(\mathrm{X}^{\mathrm{M}}, \sigma^{*}\right)$. For any $x \in \mathrm{X}, \lambda \in \mathfrak{a}_{\mathrm{M}, \mathbf{C}}^{*}$ and $\varphi \in \mathrm{I}(\sigma)$ consider the expression

$$
\mathrm{J}_{\mathrm{M}}(\varphi: x, \alpha, \lambda)=\sum_{y} v_{\mathrm{M}}(y) e^{\frac{1}{2}\left\langle\rho_{\mathrm{M}}+\lambda, \mathrm{H}(y)\right\rangle} \int_{\mathrm{P}^{y} \backslash \mathrm{G} y} \alpha_{y}\left(\varphi_{\lambda}\left(g \iota_{y}^{x}\right)\right) d g
$$

where $y$ ranges over a (finite) set of representatives of the M-orbits $(\mathrm{M} \cap x \bullet \mathrm{G}) / \mathrm{M}$ in $\mathrm{M} \cap$ $x \bullet \mathrm{G}$ and $\iota_{y}^{x} \in \mathrm{G}$ is such that $x=y \bullet \iota_{y}^{x}$. (See Lemma 4.3 below for geometric motivation.)

Proposition 4.1. - Let $\sigma \in \mathcal{R}(\mathrm{M})$ and $\alpha \in \mathcal{E}_{\mathrm{M}}\left(\mathrm{X}^{\mathrm{M}}, \sigma^{*}\right)$. In the Archimedean case, assume further that $\sigma$ is unitarizable. Then

(1) The sum of integrals $(\mathbf{4 . 2})$ is well-defined and absolutely convergent for $\lambda=\left(\lambda_{1}, \ldots, \lambda_{t}\right) \in$ $\mathfrak{a}_{\mathrm{M}, \mathbf{C}}^{*}$ such that $\operatorname{Re}\left(\lambda_{i}-\lambda_{i+1}\right) \gg 0, i=1, \ldots, t-1$.

(2) For every $x \in \mathrm{X}$ the linear form $\varphi \mapsto \mathrm{J}_{\mathrm{M}}^{\mathrm{G}}(\varphi: x, \alpha, \lambda)$ admits a meromorphic continuation in $\lambda \in \mathfrak{a}_{\mathrm{M}, \mathbf{G}}^{*}$ (still denoted by $\left.\mathrm{J}(x, \alpha, \lambda)=\mathrm{J}_{\mathrm{M}}^{\mathrm{G}}(x, \alpha, \lambda)\right)$. In the $p$-adic case, it is a rational function in $q_{\mathrm{F}}^{\lambda}=\left(q_{\mathrm{F}}^{\lambda_{1}}, \ldots, q_{\mathrm{F}}^{\lambda_{t}}\right)$. In the Archimedean case, it has hyperplane singularities of the form $\lambda_{i}-\lambda_{j}=c$.

(3) The map $x \mapsto \mathrm{J}(x, \alpha, \lambda)$ is an element of $\mathcal{E}_{\mathrm{G}}\left(\mathrm{X}, \mathrm{I}(\sigma, \lambda)^{*}\right)$ which we denote by $\mathrm{J}(\alpha, \lambda)$.

(4) Suppose that $\mathrm{J}(x, \alpha, \cdot)$ is regular at $\lambda$. Then $\mathrm{J}(x, \alpha, \lambda) \equiv 0$ if and only if $\alpha_{y} \equiv 0$ for all $y \in \mathrm{M} \cap x \bullet \mathrm{G}$.

Proof. - We first check that the definition formally makes sense. If $y=$ $\operatorname{diag}\left(y_{1}, \ldots, y_{t}\right) \in \mathrm{X}^{\mathrm{M}}$ then

$$
\mathrm{P}^{y}=\mathrm{M}^{y}=\left\{\operatorname{diag}\left(g_{1}, \ldots, g_{t}\right): g_{i} \in \mathrm{G}_{n_{i}}^{y_{i}}, i=1, \ldots, t\right\}
$$

is a product of unitary groups in the diagonal blocks of $\mathrm{M}$. The measure on $\mathrm{P}^{y}$ is the product of the measures on $\mathrm{G}_{n_{i}}^{y_{i}}$ according to our convention. It follows from the description of $\mathrm{P}^{y}$ that $\mathrm{H}(m)_{\mathrm{M}}=0$ for all $m \in \mathrm{P}^{y}$. Furthermore, since $\alpha_{y}$ is an $\mathrm{M}^{y}$-invariant linear form on $\sigma$ we have

$$
\alpha_{y}\left(\varphi_{\lambda}\left(m g \iota_{y}^{x}\right)\right)=\alpha_{y}\left(\sigma(m) \varphi_{\lambda}\left(g \iota_{y}^{x}\right)\right)=\alpha_{y}\left(\varphi_{\lambda}\left(g \iota_{y}^{x}\right)\right), \quad m \in \mathrm{P}^{y}, g \in \mathrm{G}
$$


and therefore, the integrand in (4.2) is indeed $\mathrm{M}^{y}$-invariant. If $\iota \in \mathrm{G}$ is another element such that $x=y \bullet \iota$ then $\iota \in \mathrm{G}^{y} \iota_{y}^{x}$ and the $\mathrm{G}^{y}$-invariance of $d g$ shows that the integral is independent of the choice of $\iota_{y}^{x}$. If $m \in \mathbf{M}$ then we can choose $\iota_{y \bullet m}^{x}=m^{-1} \iota_{y}^{x}$ and the equivariance of $\alpha$ gives that

$$
\begin{aligned}
e^{\frac{1}{2}\left\langle\rho_{\mathrm{M}}+\lambda, \mathrm{H}(y \bullet m)\right\rangle} \alpha_{y \bullet m}\left(\varphi_{\lambda}\left(g m^{-1} \iota_{y}^{x}\right)\right) & =e^{\frac{1}{2}\left\langle\rho_{\mathrm{M}}+\lambda, \mathrm{H}(y)+2 \mathrm{H}(m)\right\rangle} \alpha_{y}\left(\sigma(m) \varphi_{\lambda}\left(g m^{-1} \iota_{y}^{x}\right)\right) \\
& =e^{\frac{1}{2}\left\langle\rho_{\mathrm{M}}+\lambda, \mathrm{H}(y)\right\rangle} \alpha_{y}\left(\varphi_{\lambda}\left(m g m^{-1} \iota_{y}^{x}\right)\right)
\end{aligned}
$$

for all $g \in \mathrm{G}$. Since $\mathrm{G}^{y \bullet m}=m^{-1} \mathrm{G}^{y} m$ it follows, that

$$
\begin{aligned}
& e^{\frac{1}{2}\left\langle\rho_{\mathrm{M}}+\lambda, \mathrm{H}(y \bullet m)\right\rangle} \int_{\mathrm{N}^{\bullet \bullet} \backslash \mathrm{G}^{\bullet \bullet m}} \alpha_{y \bullet m}\left(\varphi_{\lambda}\left(g \iota_{y \bullet m}^{x}\right)\right) d g \\
& =e^{\frac{1}{2}\left\langle\rho_{\mathrm{M}}+\lambda, \mathrm{H}(y)\right\rangle} \int_{\mathrm{M}^{\bullet} \backslash \mathrm{G}^{\bullet \bullet m}} \alpha_{y}\left(\varphi_{\lambda}\left(m g m^{-1} \iota_{y}^{x}\right)\right) d g \\
& =e^{\frac{1}{2}\left\langle\rho_{\mathrm{M}}+\lambda, \mathrm{H}(y)\right\rangle} \int_{\mathrm{M}^{y} \backslash \mathrm{G}^{y}} \alpha_{y}\left(\varphi_{\lambda}\left(g l_{y}^{x}\right)\right) d g .
\end{aligned}
$$

Since we also have $v_{\mathrm{M}}(y \bullet m)=v_{\mathrm{M}}(y), y \in \mathrm{X}^{\mathrm{M}}, m \in \mathrm{M}$, we see that each summand in (4.2) is independent of the choice of a representative $y$ of the M-orbit and whenever absolutely convergent, (4.2) is well-defined. Furthermore, for any $h \in \mathrm{G}$ we may choose $\iota_{y}^{x \bullet h}=\iota_{y}^{x} h$. Since

$$
\varphi_{\lambda}\left(g \iota_{y}^{x} h\right)=(\mathrm{I}(h, \sigma, \lambda) \varphi)_{\lambda}\left(g \iota_{y}^{x}\right),
$$

it is also formal from the definition that whenever defined by an absolutely convergent sum of integrals, we have

$$
\mathrm{J}(\varphi: x \bullet g, \alpha, \lambda)=\mathrm{J}(\mathrm{I}(g, \sigma, \lambda) \varphi: x, \alpha, \lambda), \quad g \in \mathrm{G} .
$$

Next, we justify the absolute convergence and meromorphic continuation. The absolute convergence of integrals of the form

$$
\int_{\mathrm{M}^{y} \backslash \mathrm{G}^{y}} \alpha_{y}\left(\varphi_{\lambda}(g \iota)\right) d g
$$

in some positive cone and their meromorphic continuation (to a rational function in $q_{\mathrm{F}}^{\lambda}$ in the non-Archimedean case) was proved in a more general context of a reductive symmetric space. In the Archimedean case this was done by Brylinski, Carmona and Delorme ([CD94, Proposition 2 and Theorem 3], cf. [BD92]) using a result of Flensted-Jensen, Öshima and Schlichtkrull [FJŌS88]. In the non-Archimedean case we refer to BlancDelorme [BD08, Theorems 2.8 and 2.16]. ${ }^{6}$ The result in [loc. cit.] is stated under an additional assumption, but the latter is always satisfied thanks to a result of Lagier [Lag08,

\footnotetext{
${ }^{6}$ Note that in the terminology of [loc. cit.], any $\mathrm{P}$ is a $\theta_{y}$-parabolic subgroup of G where $\theta_{y}(g)=y^{-1}\left({ }^{t} g^{\tau}\right)^{-1} y$.
} 
Theorem 4(i)]. (Alternatively, one could also prove the rationality using Bernstein's meromorphic continuation principle as in the proof of [LR00, Proposition 2] - cf. Remark 6.8 below.) The formal steps at the beginning of the proof are now justified.

For the last part, let $\mathrm{G}^{\circ}[x]=\left\{g \in \mathrm{G}: x \bullet g^{-1} \in \mathrm{X}^{\circ}\right\}$ be the union of open double cosets in $\mathrm{P} \backslash \mathrm{G} / \mathrm{G}^{x}$. One observes (cf. [CD94, Theorem 3, part 3]) that the restriction of $\mathrm{J}(x, \alpha, \lambda)$ to

$$
\left\{\varphi \in \mathrm{I}(\sigma): \operatorname{supp} \varphi \subseteq \mathrm{G}^{\circ}[x]\right\}
$$

is non-zero if and only if there exists $y \in \mathrm{X}^{\mathrm{M}} \cap x \bullet \mathrm{G}$ such that $\alpha_{y} \not \equiv 0$.

Corollary 4.2. - Let $\sigma \in \mathcal{R}(\mathrm{M}), \pi=\mathrm{I}(\sigma, \lambda)$ and $x \in \mathrm{X}$. In the Archimedean case assume further that $\sigma$ is unitarizable. Suppose that $\sigma$ is $\mathrm{M}^{y}$-distinguished for some $y \in \mathrm{M} \cap x \bullet \mathrm{G}$. Then $\pi$ is $\mathrm{G}^{x}$-distinguished.

Proof. - The Corollary follows by taking $\alpha \in \mathcal{E}_{\mathrm{M}}\left(\mathrm{X}^{\mathrm{M}}, \sigma^{*}\right)$ non-zero and supported (as a function on $\mathrm{X}^{\mathrm{M}}$ ) in the given M-orbit $y \bullet \mathrm{M}$ and taking the leading term at $\lambda$ of the restriction of $\mathrm{J}_{\mathrm{M}}(x, \alpha, \cdot)$ to a complex line through $\lambda$ in general position.

We may interpret and motivate the definition of the operation $\mathrm{J}_{\mathrm{M}}$ as follows. Recall the isomorphism

$$
\mathcal{E}_{\mathrm{G}}\left(\mathrm{X}, \mathrm{I}(\sigma, \lambda)^{*}\right) \rightarrow \operatorname{Hom}_{\mathrm{G}}\left(\mathcal{S}(\mathrm{X}), \mathrm{I}(\sigma, \lambda)^{\vee}\right)
$$

defined by (2.6). Similarly, for $\alpha \in \mathcal{E}_{\mathrm{M}}\left(\mathrm{X}^{\mathrm{M}}, \sigma^{*}\right)$ we may define $\Phi \odot \alpha$ by

$$
(\Phi \odot \alpha)(v)=\int_{\mathrm{X}^{\mathrm{M}}} v_{\mathrm{M}}(y) \Phi(y) \alpha_{y}(v) d y, \quad \Phi \in \mathcal{S}\left(\mathrm{X}^{\mathrm{M}}\right), v \in \sigma .
$$

(Note that this is consistent with $(\mathbf{2 . 6})$ when $\mathrm{M}=\mathrm{G}$.) Then $\alpha \mapsto(\Phi \mapsto \Phi \odot \alpha)$ defines an isomorphism of vector spaces

$$
\mathcal{E}_{\mathrm{M}}\left(\mathrm{X}^{\mathrm{M}}, \sigma^{*}\right) \rightarrow \operatorname{Hom}_{\mathrm{M}}\left(\mathcal{S}\left(\mathrm{X}^{\mathrm{M}}\right), \sigma^{\vee}\right) .
$$

We also have a canonical isomorphism of vector spaces

$$
\mathcal{E}_{\mathrm{M}}\left(\mathrm{X}^{\mathrm{M}}, \sigma^{*}\right) \rightarrow \mathcal{E}_{\mathrm{M}}\left(\mathrm{X}^{\mathrm{M}}, \sigma[\lambda]^{*}\right)
$$

for any $\lambda \in \mathfrak{a}_{\mathrm{M}, \mathbf{G}}^{*}$ given by $\alpha \mapsto \alpha[\lambda]$ where

$$
\alpha[\lambda]_{y}=e^{\frac{1}{2}\langle\lambda, \mathrm{H}(y)\rangle} \alpha_{y}, \quad y \in \mathrm{X}^{\mathrm{M}} .
$$

The map $\alpha \mapsto \mathrm{J}_{M}(\alpha, \lambda)$ (when defined) gives rise to a map

$$
\mathrm{J}_{\mathrm{M}}(\lambda): \mathcal{E}_{\mathrm{M}}\left(\mathrm{X}^{\mathrm{M}}, \sigma^{*}\right) \rightarrow \mathcal{E}_{\mathrm{G}}\left(\mathrm{X}, \mathrm{I}(\sigma, \lambda)^{*}\right)
$$


and therefore, via the identifications above, gives rise to a map

$$
\operatorname{Hom}_{\mathrm{M}}\left(\mathcal{S}\left(\mathrm{X}^{\mathrm{M}}\right), \sigma^{\vee}[-\lambda]\right) \rightarrow \operatorname{Hom}_{\mathrm{G}}\left(\mathcal{S}(\mathrm{X}), \mathrm{I}(\sigma, \lambda)^{\vee}\right)
$$

By Frobenius reciprocity, we obtain a map

$$
\operatorname{Hom}_{M}\left(\mathcal{S}\left(\mathrm{X}^{\mathrm{M}}\right), \sigma^{\vee}[-\lambda]\right) \rightarrow \operatorname{Hom}_{\mathrm{M}}\left(r_{\mathrm{M}}(\mathcal{S}(\mathrm{X})), \sigma^{\vee}[-\lambda]\right) .
$$

For any $\Phi \in \mathcal{S}(\mathrm{X})$ let $\Phi^{\mathrm{M}}$ be the smooth function on $\mathrm{X}^{\mathrm{M}}$ defined by

$$
\Phi^{\mathrm{M}}(y)=e^{\frac{1}{2}\left\langle\rho_{\mathrm{M}}, \mathrm{H}(y)\right\rangle} \int_{\mathrm{U}} \Phi(y \bullet u) d u .
$$

In general, $\Phi^{\mathrm{M}} \notin \mathcal{S}\left(\mathrm{X}^{\mathrm{M}}\right)$. However, we have $\Phi^{\mathrm{M}} \in \mathcal{S}\left(\mathrm{X}^{\mathrm{M}}\right)$ for any $\Phi \in \mathcal{S}\left(\mathrm{X}^{\circ}\right)$ where we identify $\mathcal{S}\left(\mathrm{X}^{\circ}\right)$ with the P-invariant subspace of $\mathcal{S}(\mathrm{X})$ consisting of functions which vanish (together with all their derivatives in the Archimedean case) on the complement of $\mathrm{X}^{\circ}$. We get an M-equivariant map

$$
r_{\mathrm{M}}\left(\mathcal{S}\left(\mathrm{X}^{\circ}\right)\right) \rightarrow \mathcal{S}\left(\mathrm{X}^{\mathrm{M}}\right)
$$

The alternative description of $\mathrm{J}_{M}$ is as follows.

Lemma 4.3. - The composition of $(\mathbf{4 . 3})$ with the restriction map

$$
\operatorname{Hom}_{\mathrm{M}}\left(r_{\mathrm{M}}(\mathcal{S}(\mathrm{X})), \sigma^{\vee}[-\lambda]\right) \rightarrow \operatorname{Hom}_{\mathrm{M}}\left(r_{\mathrm{M}}\left(\mathcal{S}\left(\mathrm{X}^{\circ}\right)\right), \sigma^{\vee}[-\lambda]\right)
$$

coincides with the map

$$
\operatorname{Hom}_{M}\left(\mathcal{S}\left(\mathrm{X}^{\mathrm{M}}\right), \sigma^{\vee}[-\lambda]\right) \rightarrow \operatorname{Hom}_{\mathrm{M}}\left(r_{\mathrm{M}}\left(\mathcal{S}\left(\mathrm{X}^{\circ}\right)\right), \sigma^{\vee}[-\lambda]\right)
$$

obtained by composition with the map (4.4) induced by $\Phi \mapsto \Phi^{\mathrm{M}}$.

Proof. - Explicating the various identifications, the Lemma amounts to the relation

$$
\left(\Phi \odot \mathrm{J}_{\mathrm{M}}(\alpha, \lambda)\right)(\varphi)=\int_{\mathrm{P} \backslash \mathrm{G}}\left((\mathrm{R}(g) \Phi)^{\mathrm{M}} \odot \alpha[\lambda]\right)(\varphi(g)) d g
$$

for any $\varphi \in \mathrm{I}(\sigma)$ and $\Phi \in \mathcal{S}\left(\mathrm{X}^{\circ}\right)$.

To show this, we compute the left hand side as

$$
\begin{aligned}
\int_{\mathrm{X}} & \Phi(x) \mathrm{J}_{\mathrm{M}}(\varphi: x, \alpha, \lambda) d x \\
& =\int_{\mathrm{X}} \Phi(x) \sum_{y \in(x \bullet \mathrm{G} \cap \mathrm{M}) / \mathrm{M}} v_{\mathrm{M}}(y) e^{\frac{1}{2}\left\langle\rho_{\mathrm{M}}+\lambda, \mathrm{H}(y)\right\rangle} \int_{\mathrm{P}^{y} \backslash \mathrm{G}^{y}} \alpha_{y}\left(\varphi_{\lambda}\left(g \iota_{y}^{x}\right)\right) d g d x
\end{aligned}
$$




$$
\begin{aligned}
& =\sum_{y \in \mathrm{X}^{\mathrm{M}} / \mathrm{M}} v_{\mathrm{M}}(y) e^{\frac{1}{2}\left\langle\rho_{\mathrm{M}}+\lambda, \mathrm{H}(y)\right\rangle} \int_{\mathrm{G}^{y} \backslash \mathrm{G}} \Phi(y \bullet z) \int_{\mathrm{P}^{y} \backslash \mathrm{G}^{y}} \alpha_{y}\left(\varphi_{\lambda}(g z)\right) d g d z \\
& =\sum_{y \in \mathrm{X}^{\mathrm{M}} / \mathrm{M}} v_{\mathrm{M}}(y) e^{\frac{1}{2}\left\langle\rho_{\mathrm{M}}+\lambda, \mathrm{H}(y)\right\rangle} \int_{\mathrm{P}^{y} \backslash \mathrm{G}} \Phi(y \bullet g) \alpha_{y}\left(\varphi_{\lambda}(g)\right) d g \\
& =\sum_{y \in \mathrm{X}^{\mathrm{M}} / \mathrm{M}} v_{\mathrm{M}}(y) e^{\frac{1}{2}\left\langle\rho_{\mathrm{M}}+\lambda, \mathrm{H}(y)\right\rangle} \int_{\mathrm{M} \backslash \mathrm{G}} \int_{\mathrm{M}^{\gamma} \backslash \mathrm{M}} \Phi(y \bullet m g) \alpha_{y}\left(\varphi_{\lambda}(m g)\right) d m d g \\
& =\sum_{y \in \mathrm{X}^{\mathrm{M}} / \mathrm{M}} v_{\mathrm{M}}(y) e^{\frac{1}{2}\left\langle\rho_{\mathrm{M}}+\lambda, \mathrm{H}(y)\right\rangle} \\
& \times \int_{\mathrm{P} \backslash \mathrm{G}} \int_{\mathrm{U}} \int_{\mathrm{M}^{y} \backslash \mathrm{M}} \Phi(y \bullet m u g) \alpha_{y}\left(\varphi_{\lambda}(m u g)\right) d m d u d g \\
& =\sum_{y \in \mathrm{X}^{\mathrm{M}} / \mathrm{M}} v_{\mathrm{M}}(y) e^{\frac{1}{2}\left\langle\rho_{\mathrm{M}}+\lambda, \mathrm{H}(y)\right\rangle} \\
& \times \int_{\mathrm{P} \backslash \mathrm{G}} \int_{\mathrm{M}^{\gamma} \backslash \mathrm{M}} e^{-\frac{1}{2}\left\langle\rho_{\mathrm{M}}, \mathrm{H}(y \bullet m)\right\rangle}(\mathrm{R}(g) \Phi)^{\mathrm{M}}(y \bullet m) \alpha_{y}\left(\varphi_{\lambda}(m g)\right) d m d g \\
& =\int_{\mathrm{P} \backslash \mathrm{G}} \sum_{y \in \mathrm{X}^{\mathrm{M}} / \mathrm{M}} \int_{\mathrm{M}^{\gamma} \backslash \mathrm{M}} v_{\mathrm{M}}(y) e^{\frac{1}{2}\langle\lambda, \mathrm{H}(y \bullet m)\rangle}(\mathrm{R}(g) \Phi)^{\mathrm{M}}(y \bullet m) \alpha_{y \bullet m}\left(\varphi_{\lambda}(g)\right) d m d g \\
& =\int_{\mathrm{P} \backslash \mathrm{G}} \int_{\mathrm{X}^{\mathrm{M}}} v_{\mathrm{M}}(y) e^{\frac{1}{2}\langle\lambda, \mathrm{H}(y)\rangle}(\mathrm{R}(g) \Phi)^{\mathrm{M}}(y) \alpha_{y}\left(\varphi_{\lambda}(g)\right) d y d g \\
& =\int_{\mathrm{P} \backslash \mathrm{G}}\left((\mathrm{R}(g) \Phi)^{\mathrm{M}} \odot \alpha[\lambda]\right)(\varphi(g)) d g
\end{aligned}
$$

where the integrals are absolutely convergent for $\Phi \in \mathcal{S}\left(\mathrm{X}^{\circ}\right)$. The lemma follows.

It will be useful to normalize the functionals $\mathrm{J}_{\mathrm{M}}$ for $\sigma \in \mathcal{R}_{\mathrm{pi}}(\mathrm{M})$ (assumed unitarizable in the Archimedean case). The normalizing factor depends on an auxiliary $\sigma^{\prime} \in \mathcal{R}_{\mathrm{pi}}\left(\mathrm{M}^{\prime}\right)$ (as well as on $\sigma$ itself which will be omitted from the notation, since in all cases at hand, $\sigma$ will be determined by $\sigma^{\prime}$ ). More precisely, for $\lambda \in \mathfrak{a}_{\mathrm{M}, \mathbf{C}}^{*}$ set

$$
\mathfrak{n}\left(\sigma^{\prime}, \lambda\right)=\mathfrak{n}_{\mathrm{M}^{\prime}}\left(\sigma^{\prime}, \lambda\right)=\frac{\mathrm{C}_{\mathrm{M}}\left(w_{\mathrm{M}}: \sigma, \lambda ; \psi\right)}{\mathrm{C}_{\mathrm{M}^{\prime}}\left(w_{\mathrm{M}}: \sigma^{\prime}, \lambda ; \psi^{\prime}\right)}
$$

and define the normalized linear forms and equivariant maps

$$
\begin{aligned}
& \mathcal{J}_{\sigma^{\prime}}(x, \alpha, \lambda)=\mathfrak{n}\left(\sigma^{\prime}, \lambda\right) \mathrm{J}(x, \alpha, \lambda), \quad x \in \mathrm{X} \\
& \mathcal{J}_{\sigma^{\prime}}(\alpha, \lambda)=\mathfrak{n}\left(\sigma^{\prime}, \lambda\right) \mathrm{J}(\alpha, \lambda) .
\end{aligned}
$$

By Proposition 4.1, these are meromorphic functions in $\lambda$ (rational in $q_{\mathrm{F}}^{\lambda}$ in the nonArchimedean case) and lie in $\operatorname{Hom}_{\mathrm{G}^{x}}(\mathrm{I}(\sigma, \lambda), \mathbf{C})$ and $\mathcal{E}_{\mathrm{G}}\left(\mathrm{X}, \mathrm{I}(\sigma, \lambda)^{*}\right)$ respectively. Sup- 
pose further that $\sigma^{\prime} \in \Pi^{\mathrm{M}^{\prime}}$ and $\sigma=\mathrm{bc}\left(\sigma^{\prime}\right)$. In this case, we may write

$$
\mathfrak{n}_{\mathrm{M}^{\prime}}\left(\sigma^{\prime}, \lambda\right)=\prod_{1 \leq i<j \leq t} \lambda_{\psi^{\prime}}^{-n_{i} n_{j}} \omega_{\sigma_{j}^{\prime}}(-1)^{n_{i}} \gamma\left(\lambda_{i}-\lambda_{j}, \sigma_{i}^{\prime} \times \sigma_{j}^{\prime \vee} \cdot \eta ; \psi\right)
$$

Note that it follows from $(\mathbf{3 . 1 2})$ that in the notation of Lemma 3.7, for any $\sigma^{\prime} \in \Pi_{\mathrm{bc} \text {-gen }}^{\mathrm{M}^{\prime}}$ we have

$$
\mathcal{J}_{\sigma^{\prime}[\mu]}\left(\alpha^{\sigma^{\prime}[\mu]}, \lambda\right)=\mathcal{J}_{\sigma^{\prime}}\left(\alpha^{\sigma^{\prime}}, \mu+\lambda\right) \circ \mathcal{A}(\sigma, \mu)
$$

where $\sigma=\operatorname{bc}\left(\sigma^{\prime}\right)$ and $\mu, \lambda \in \mathfrak{a}_{\mathrm{M}, \mathbf{G}}^{*}$.

For inductive arguments, it will be useful to consider the open periods in the relative situation for a pair $\mathrm{L}=\mathrm{M}_{\gamma} \subseteq \mathrm{M}=\mathrm{M}_{\kappa}$ of standard Levi subgroups in G. Let $\nu_{\mathrm{L}}^{\mathrm{M}}(\ell)$ be defined for $\ell$ in either $\mathrm{L}^{\prime}$ or $\mathrm{X}^{\mathrm{L}}$ by

$$
v_{\mathrm{L}}(\ell)=v_{\mathrm{L}}^{\mathrm{M}}(\ell) v_{\mathrm{M}}(\ell)
$$

For a representation $\varrho \in \mathcal{R}(\mathrm{L})$ (unitarizable in the Archimedean case), $y \in \mathrm{X}^{\mathrm{M}}$ and $\alpha \in$ $\mathcal{E}_{\mathrm{L}}\left(\mathrm{X}^{\mathrm{L}}, \varrho^{*}\right)$, we define the linear form $\mathrm{J}_{\mathrm{L}}^{\mathrm{M}}(y, \alpha, \mu) \in \operatorname{Hom}_{\mathrm{M}^{y}}\left(\mathrm{I}_{\mathrm{L}}^{\mathrm{M}}(\varrho, \mu), \mathbf{C}\right)$ as the function in $\mu \in \mathfrak{a}_{\mathrm{L}, \mathbf{G}}^{*}$ given by the meromorphic continuation of the sum of integrals

$$
\mathrm{J}_{\mathrm{L}}^{\mathrm{M}}(\varphi: y, \alpha, \mu)=\sum_{z \in(\mathrm{L} \cap y \bullet \mathrm{M}) / \mathrm{L}} v_{\mathrm{L}}^{\mathrm{M}}(z) e^{\frac{1}{2}\left\langle\rho_{\mathrm{L}}^{\mathrm{M}}+\mu, \mathrm{H}(z)\right\rangle} \int_{\mathrm{L}^{z} \backslash \mathrm{M}^{z}} \alpha_{z}\left(\varphi_{\mu}\left(m \iota_{z}^{y}\right)\right) d m
$$

for $\varphi \in \mathrm{I}_{\mathrm{L}}^{\mathrm{M}}(\sigma)$, where $\iota_{z}^{y} \in \mathrm{M}$ is a choice such that $z \bullet \iota_{z}^{y}=y$. We denote by $\mathrm{J}_{\mathrm{L}}^{\mathrm{M}}(\alpha, \mu) \in$ $\mathcal{E}_{\mathrm{M}}\left(\mathrm{X}^{\mathrm{M}}, \mathrm{I}_{\mathrm{L}}^{\mathrm{M}}(\sigma, \mu)^{*}\right)$ the associated equivariant map. Assume that $\varrho \in \mathcal{R}_{\mathrm{pi}}(\mathrm{L})$ and let $\varrho^{\prime}=$ $\varrho_{1}^{\prime} \otimes \cdots \otimes \varrho_{t}^{\prime} \in \Pi^{\mathrm{L}^{\prime}}$ where $\varrho_{i}^{\prime}$ is a representation of the Levi $\mathrm{M}_{\gamma_{i}}^{\prime}$ of $\mathrm{G}_{n_{i}}^{\prime}$ (see Section 1 for the notation). Let $\mu=\left(\mu_{1}, \ldots, \mu_{t}\right) \in \mathfrak{a}_{\mathrm{L}, \mathbf{G}}^{*}$ with $\mu_{i} \in \mathfrak{a}_{\mathrm{M}_{\gamma_{i}}, \mathbf{G}}$ and let $w \in \mathrm{W}^{\mathrm{M}}(\mathrm{L})$. Writing $w=\operatorname{diag}\left(w_{1}, \ldots, w_{t}\right)$ where $w_{i} \in \mathrm{W}^{\mathrm{G}_{n_{i}}}\left(\mathrm{M}_{\gamma_{i}}\right)$, we set

$$
\begin{gathered}
\mathrm{C}_{\mathrm{L}^{\prime}}^{\mathrm{M}^{\prime}}\left(w: \varrho^{\prime}, \mu ; \psi^{\prime}\right)=\prod_{i=1}^{t} \mathrm{C}_{\mathrm{M}_{\gamma_{i}}^{\prime}}^{\mathrm{G}_{n_{i}}^{\prime}}\left(w_{i}: \varrho_{i}^{\prime}, \mu_{i} ; \psi^{\prime}\right), \\
\mathrm{C}_{\mathrm{L}}^{\mathrm{M}}(w: \varrho, \mu ; \psi)=\prod_{i=1}^{t} \mathrm{C}_{\mathrm{M}_{\gamma_{i}}}^{\mathrm{G}_{n_{i}}}\left(w_{i}: \varrho_{i}, \mu_{i} ; \psi\right),
\end{gathered}
$$

and

$$
\mathfrak{n}_{\mathrm{L}^{\prime}}^{\mathrm{M}^{\prime}}\left(\varrho^{\prime}, \mu\right)=\frac{\mathrm{C}_{\mathrm{L}}^{\mathrm{M}}\left(w_{\mathrm{M}}^{\mathrm{L}}: \varrho, \mu ; \psi\right)}{\mathrm{C}_{\mathrm{L}^{\prime}}^{\mathrm{M}^{\prime}}\left(w_{\mathrm{M}}^{\mathrm{L}}: \varrho^{\prime}, \mu ; \psi^{\prime}\right)}
$$


It follows from the multiplicativity of $\gamma$-factors $(\mathbf{1 . 8})$ that for any $w_{1} \in \mathrm{W}(\mathrm{M}), w_{2} \in$ $\mathrm{W}^{\mathrm{M}}(\mathrm{L})$ we have

$$
\mathrm{C}_{\mathrm{L}^{\prime}}\left(w_{1} w_{2}: \varrho^{\prime}, \mu+\lambda ; \psi^{\prime}\right)=\mathrm{C}_{\mathrm{L}^{\prime}}^{\mathrm{M}^{\prime}}\left(w_{2}: \varrho^{\prime}, \mu ; \psi^{\prime}\right) \mathrm{C}_{\mathrm{M}^{\prime}}\left(w_{1}: \mathrm{I}_{\mathrm{L}^{\prime}}^{\mathrm{M}^{\prime}}\left(\varrho^{\prime}, \mu\right), \lambda ; \psi^{\prime}\right)
$$

as meromorphic functions in $\mu \in \mathfrak{a}_{\mathrm{L}, \mathbf{G}}^{*}$ and $\lambda \in \mathfrak{a}_{\mathrm{M}, \mathbf{G}}^{*}$. On $\mathrm{I}_{\mathrm{L}}^{\mathrm{M}}(\varrho)$ we set

$$
\mathcal{J}_{\mathrm{L}, \varrho^{\prime}}^{\mathrm{M}}(x, \alpha, \mu)=\mathfrak{n}_{\mathrm{L}^{\prime}}^{\mathrm{M}^{\prime}}\left(\varrho^{\prime}, \mu\right) \mathrm{J}_{\mathrm{L}}^{\mathrm{M}}(x, \alpha, \mu)
$$

We now show that the open local unitary periods are compatible with transitivity of induction.

Lemma 4.4. - Let $\mathrm{L} \subseteq \mathrm{M}$ be Levi subgroups of $\mathrm{G}, \varrho \in \mathcal{R}(\mathrm{L}), \varrho^{\prime} \in \Pi^{\mathrm{L}^{\prime}}$ and $\alpha \in$ $\mathcal{E}_{\mathrm{L}}\left(\mathrm{X}^{\mathrm{L}}, \varrho^{*}\right)$. In the Archimedean case, assume further that $\varrho$ is unitarizable. Then as a meromorphic function in $\lambda+\mu$ (in the $p$-adic case, rational function in $q_{\mathrm{F}}^{\mu+\lambda}$ ) for $\mu \in \mathfrak{a}_{\mathrm{L}, \mathbf{G}}^{*}$ and $\lambda \in \mathfrak{a}_{\mathrm{M}, \mathbf{G}}^{*}$, we have

$$
\mathrm{J}_{\mathrm{L}}^{\mathrm{G}}(x, \alpha, \mu+\lambda)=\mathrm{J}_{\mathrm{M}}^{\mathrm{G}}\left(x, \mathrm{~J}_{\mathrm{L}}^{\mathrm{M}}(\alpha, \mu), \lambda\right) \circ \Gamma_{\mathrm{L}, \mathrm{M}}(\varrho, \mu)
$$

and if $\varrho \in \mathcal{R}_{\mathrm{pi}}(\mathrm{L})$ then similarly

$$
\mathcal{J}_{\mathrm{L}, \varrho^{\prime}}^{\mathrm{G}}(x, \alpha, \mu+\lambda)=\mathcal{J}_{\mathrm{M}, \mathrm{I}_{\mathrm{L}^{\prime}}^{\mathrm{M}^{\prime}}\left(\varrho^{\prime}, \mu\right)}^{\mathrm{G}}\left(x, \mathcal{J}_{\mathrm{L}, \varrho^{\prime}}^{\mathrm{M}}(\alpha, \mu), \lambda\right) \circ \Gamma_{\mathrm{L}, \mathrm{M}}(\varrho, \mu) .
$$

The right-hand sides of $(\mathbf{4 . 8})$ and $(\mathbf{4 . 9})$ are defined by an absolutely convergent sum of integrals (also in the Archimedean case) whenever $\lambda$ and $\mu$ are sufficiently positive and by meromorphic continuation in general.

Proof. - Since $w_{\mathrm{L}}=w_{\mathrm{M}} w_{\mathrm{L}}^{\mathrm{M}},(\mathbf{4 . 9})$ follows from $(\mathbf{4 . 7})$ and $(\mathbf{4 . 8})$. To prove the identity $(\mathbf{4 . 8})$ we may assume that $\operatorname{Re} \mu$ and $\operatorname{Re} \lambda$ are sufficiently regular in the corresponding cones. A set of representatives for $(\mathrm{L} \cap x \bullet \mathrm{G}) / \mathrm{L}$ can be chosen by first fixing a set $\{y\}$ of representatives for $(\mathrm{M} \cap x \bullet \mathrm{G}) / \mathrm{M}$ and then taking the union over all such $y$ of a set of representatives for $(\mathrm{L} \cap y \bullet \mathrm{M}) / \mathrm{L}$. By Proposition 4.1 for any $\varphi \in \mathrm{I}_{\mathrm{L}}(\varrho), \mathrm{J}_{\mathrm{L}}^{\mathrm{G}}(\varphi: x, \alpha, \mu+\lambda)$ is equal to

$$
\sum_{y \in(\mathrm{M} \cap x \bullet \mathrm{G}) / \mathrm{M}} \sum_{z \in(\mathrm{L} \cap y \bullet \mathrm{M}) / \mathrm{L}} v_{\mathrm{L}}(z) e^{\frac{1}{2}\left\langle\rho_{\mathrm{L}}+\mu+\lambda, \mathrm{H}(z)\right\rangle} \int_{\mathrm{L}^{z} \backslash \mathrm{G}^{z}} \alpha_{z}\left(\varphi_{\mu+\lambda}\left(g \iota_{z}^{x}\right)\right) d g
$$

where the sum-integral is absolutely convergent. Since $z \in y \bullet \mathrm{M}$, we have

$$
v_{\mathrm{M}}(z)=v_{\mathrm{M}}(y)
$$

Let $\iota_{z}^{y} \in \mathrm{M}$ and $\iota_{y}^{x} \in \mathrm{G}$ be chosen so that $\iota_{z}^{x}=\iota_{z}^{y} \iota_{y}^{x}$. Since $z \bullet \iota_{z}^{y}=y$, we have

$$
\left\langle\rho_{\mathrm{L}}+\mu+\lambda, \mathrm{H}(z)\right\rangle=\left\langle\rho_{\mathrm{L}}^{\mathrm{M}}+\mu, \mathrm{H}(z)\right\rangle+\left\langle\rho_{\mathrm{M}}+\lambda, \mathrm{H}(y)-2 \mathrm{H}\left(\iota_{z}^{y}\right)\right\rangle .
$$


Since $\left(v_{z}^{y}\right)^{-1} \mathrm{M}^{z} \boldsymbol{t}_{z}^{y}=\mathrm{M}^{y}$ and $\left(v_{z}^{y}\right)^{-1} \mathrm{G}^{z} \ell_{z}^{y}=\mathrm{G}^{y}$, by integrating in stages and performing the change of variables $g \mapsto v_{z}^{y} g\left(v_{z}^{y}\right)^{-1}$ we obtain

$$
\begin{aligned}
\int_{\mathrm{L}^{z} \backslash \mathrm{G}^{z}} \alpha_{z}\left(\varphi_{\mu+\lambda}\left(g \iota_{z}^{x}\right)\right) d g & =\int_{\mathrm{M}^{z} \backslash \mathrm{G}^{z}} \int_{\mathrm{L}^{z} \backslash \mathrm{M}^{z}} \alpha_{z}\left(\varphi_{\mu+\lambda}\left(m g \iota_{z}^{x}\right)\right) d m d g \\
& =\int_{\mathrm{M}^{y} \backslash \mathrm{G}^{y}} \int_{\mathrm{L}^{z} \backslash \mathrm{M}^{z}} \alpha_{z}\left(\varphi_{\mu+\lambda}\left(m \iota_{z}^{y} g l_{y}^{x}\right)\right) d m d g .
\end{aligned}
$$

Let $\xi=\Gamma_{\mathrm{L}, \mathrm{M}}(\varrho, \mu) \varphi$, so that by $(\mathbf{1 . 1})$ we have

$$
\varphi_{\mu+\lambda}\left(m \iota_{z}^{y} g \iota_{y}^{x}\right)=e^{\left\langle\rho_{\mathrm{M}}+\lambda, \mathrm{H}\left(m t_{z}^{y}\right)\right\rangle}\left(\xi_{\lambda}\left(g l_{y}^{x}\right)\right)_{\mu}\left(m \iota_{z}^{y}\right) .
$$

Since $\mathrm{H}(m) \in\left(\mathfrak{a}_{0}^{\mathrm{M}}\right)^{*}$ for $m \in \mathrm{M}^{z}$ we deduce that

$$
\begin{aligned}
& \int_{\mathrm{L}^{z} \backslash \mathrm{G}^{z}} \alpha_{z}\left(\varphi_{\mu+\lambda}\left(g \iota_{z}^{x}\right)\right) d g \\
& =e^{\left\langle\rho_{\mathrm{M}}+\lambda, \mathrm{H}\left(\iota_{z}^{y}\right)\right\rangle} \int_{\mathrm{M}^{y} \backslash \mathrm{G}^{y}} \int_{\mathrm{L}^{z} \backslash \mathrm{M}^{z}} \alpha_{z}\left(\left(\xi_{\lambda}\left(g \iota_{y}^{x}\right)\right)_{\mu}\left(m \iota_{z}^{y}\right)\right) d m d g .
\end{aligned}
$$

Plugging this into (4.10), taking into account (4.11) and (4.12), and changing the order of summation over $z$ with integration over $\mathrm{M}^{y} \backslash \mathrm{G}^{y}$ we obtain that

$$
\begin{aligned}
\mathrm{J}_{\mathrm{L}}^{\mathrm{G}}(\varphi: x, \alpha, \mu+\lambda)= & \sum_{y \in(\mathrm{M} \cap x \bullet \mathrm{G}) / \mathrm{M}} v_{\mathrm{M}}(y) e^{\frac{1}{2}\left\langle\rho_{\mathrm{M}}+\lambda, \mathrm{H}(y)\right\rangle} \\
& \times \int_{\mathrm{M}^{y} \backslash \mathrm{G}^{y}}\left\{\sum_{z \in\left(\mathrm{X}^{\mathrm{L}} \cap y \bullet \mathrm{M}\right) / \mathrm{L}} v_{\mathrm{L}}^{\mathrm{M}}(z) e^{\frac{1}{2}\left\langle\rho_{\mathrm{L}}^{\mathrm{M}}+\mu, \mathrm{H}(z)\right\rangle}\right. \\
& \left.\times \int_{\mathrm{L}^{z} \backslash \mathrm{M}^{z}} \alpha_{z}\left(\left(\xi_{\lambda}\left(g l_{y}^{x}\right)\right)_{\mu}\left(m \iota_{z}^{y}\right)\right) d m\right\} d g,
\end{aligned}
$$

where the right-hand side is absolutely convergent. Note that the term in curly brackets equals $\mathrm{J}_{\mathrm{L}}^{\mathrm{M}}\left(\xi_{\lambda}\left(g l_{y}^{x}\right): y, \alpha, \mu\right)$ and therefore, this gives the identity $(\mathbf{4 . 8})$.

Remark 4.5. - Under the interpretation of Lemma 4.3, Lemma 4.4 reflects the relation $\Phi^{\mathrm{L}}=\left(\Phi^{\mathrm{M}}\right)^{\mathrm{L}}$.

For future use, we define a meromorphic family of normalized relative Bessel distributions associated with a representation $\sigma^{\prime} \in \Pi_{\mathrm{bc} \text {-gen }}^{\mathrm{M}^{\prime}}$ (unitarizable in the Archimedean case) by

$$
\tilde{\mathbf{B}}\left(\sigma^{\prime}, \lambda\right)=\tilde{\mathfrak{B}}_{\mathrm{I}(\mathfrak{W}(\sigma), \lambda)}^{\mathcal{J}_{\sigma^{\prime}}\left(\alpha^{\sigma^{\prime}}, \lambda\right), \mathbf{W}_{e}\left(\sigma^{\vee},-\lambda\right)}, \quad \lambda \in \mathfrak{a}_{\mathrm{M}, \mathbf{C}}^{*}
$$

where $\sigma=\operatorname{bc}\left(\sigma^{\prime}\right)$. 


\section{Closed periods}

Next, we consider invariant functionals arising from the closed orbits of a parabolic subgroup on $\mathrm{X}$. They are defined for induced representations in special position.

5.1. Definitions and properties. - Throughout this section, let $\mathrm{L}$ be a Levi subgroup such that $w_{0} \mathrm{~L} w_{0}=\mathrm{L}$. Thus, $\mathrm{L}$ is of type $\left(n_{1}, \ldots, n_{s}, m, n_{s}, \ldots, n_{1}\right)$ where $m$ is possibly zero. We say that L is even symmetric if $m=0$ and odd symmetric otherwise. In both cases set $k=n_{1}+\cdots+n_{s}$ so that $n=2 k+m$ and let $\kappa=\left(n_{1}, \ldots, n_{s}\right)$ be the associated composition of $k$. Let

$$
\mathbf{X}_{\mathbf{L}}=\mathbf{X}_{\mathbf{L}}^{\mathbf{G}}=\mathbf{X} \cap w_{0} \mathbf{L}
$$

It is an $\mathbf{L}$-invariant subspace of $\mathbf{X}$. Let $\theta=\theta_{k}$ be the involution $\tau \circ \tilde{\theta}$ of $\mathrm{G}_{k}$ where we recall that $\tilde{\theta}(g)=w_{0}{ }^{t} g^{-1} w_{0}$. Also set

$$
w_{*}=w_{0}^{\mathrm{G}} \cdot\left(\begin{array}{ccc}
1_{k} & & \\
& w_{0}^{\mathrm{G}_{m}} & \\
& & 1_{k}
\end{array}\right)=\left(\begin{array}{ccc} 
& & w_{0}^{\mathrm{G}_{k}} \\
& 1_{m} & \\
w_{0}^{\mathrm{G}_{k}} & &
\end{array}\right) .
$$

(If $m=0$ then $w_{*}=w_{0}$.) We have

$$
\mathbf{X}_{\mathbf{L}}=\left\{w_{*} \operatorname{diag}\left(g, y, \theta\left(g^{-1}\right)\right): g \in \mathbf{M}_{\kappa}, y \in \mathbf{X}_{\boldsymbol{m}}\right\} \simeq \mathbf{M}_{\kappa} \times \mathbf{X}_{\boldsymbol{m}}
$$

with the action of $\mathbf{L}$ given by

$$
w_{*} \operatorname{diag}\left(g, y, \theta\left(g^{-1}\right)\right) \bullet \operatorname{diag}\left(h_{1}, h, \theta\left(h_{2}\right)\right)=w_{*} \operatorname{diag}\left(h_{2}^{-1} g h_{1}, y \bullet h, \theta\left(h_{2}^{-1} g h_{1}\right)^{-1}\right)
$$

for any $g, h_{1}, h_{2} \in \mathbf{M}_{\kappa}, h \in \mathbf{G}_{\boldsymbol{m}}, y \in \mathbf{X}_{\boldsymbol{m}}$. Consequently,

$$
\mathbf{L}^{\boldsymbol{w}_{*} \boldsymbol{d i a g}\left(\boldsymbol{g}, \boldsymbol{y}, \boldsymbol{\theta}\left(\boldsymbol{g}^{-\mathbf{1}}\right)\right)}=\left\{\operatorname{diag}\left(h_{1}, h, \theta\left(g h_{1} g^{-1}\right)\right): h_{1} \in \mathbf{M}_{\kappa}, h \in \mathbf{G}_{\boldsymbol{m}}^{\boldsymbol{y}}\right\} \simeq \mathbf{M}_{\kappa} \times \mathbf{G}_{\boldsymbol{m}}^{\boldsymbol{y}}
$$

Let $p_{m}: \mathbf{X}_{\mathbf{L}} \rightarrow \mathbf{X}_{\boldsymbol{m}}$ be the projection to the middle $m \times m$ block, i.e.,

$$
p_{m}\left(w_{*} \operatorname{diag}\left(g, y, \theta\left(g^{-1}\right)\right)\right)=p_{m}\left(\left(w_{w^{\mathrm{G}_{k}} g} y^{{ }^{t} g^{\tau} w_{0}^{\mathrm{G}_{k}}}\right)\right)=y .
$$

\section{Lemma 5.1.}

(1) The inclusion $\mathrm{X}_{\mathrm{L}} \subseteq \mathrm{X}$ induces an injection $\mathrm{X}_{\mathrm{L}} / \mathrm{L} \hookrightarrow \mathrm{X} / \mathrm{G}$. The image (i.e., the orbits intersecting $w_{*} \mathrm{~L}$, or equivalently $\left.w_{*} \mathrm{Q}\right)$ consists of the orbits of Witt index $\geq k$. In particular, in the even case the image is a singleton- the quasi-split forms.

(2) For any $x \in \mathrm{X}_{\mathrm{L}}, \mathrm{Q}^{x}$ is a parabolic subgroup of $\mathrm{G}^{x}$ with Levi decomposition $\mathrm{L}^{x} \mathrm{~V}^{x}$ and opposite parabolic $\left({ }^{t} \mathrm{Q}\right)^{x}$ with respect to $\mathrm{L}^{x}$. 
(3) The map p $p_{m}: \mathrm{X}_{\mathrm{L}} \rightarrow \mathrm{X}_{m}$ induces a bijection $\mathrm{X}_{\mathrm{L}} / \mathrm{L} \simeq \mathrm{X}_{m} / \mathrm{G}_{m}$. In particular, in the even case $\mathrm{X}_{\mathrm{L}}=w_{0} \bullet \mathrm{L}$.

(4) Let $\mathbf{Y}$ be the space of $n \times n$ Hermitian forms whose entries are zero on the constant coordinates of $w_{*} \mathrm{~V}$. Then for any $x \in \mathrm{X}_{\mathrm{L}}, x \bullet \mathbf{V}=x+\mathbf{Y}$ and $x \bullet \mathrm{V}=x+\mathrm{Y}$.

Proof. - Part (1) follows from Witt's cancellation theorem for Hermitian forms.

Let $x \in \mathrm{X}_{\mathrm{L}}$. Note that $\mathrm{Q}$ is invariant under the involution $g \mapsto x^{-1}{ }^{t} \tau(g)^{-1} x$. Therefore, $\mathrm{Q}^{x}$, which is the subgroup of fixed points of that involution, is a parabolic subgroup of $\mathrm{G}^{x}$. The Levi decompositions $\mathrm{Q}^{x}=\mathrm{L}^{x} \mathrm{~V}^{x},\left({ }^{t} \mathrm{Q}\right)^{x}=\mathrm{L}^{x}\left({ }^{t} \mathrm{~V}\right)^{x}$ follow from statement $(14)$ on [LR03, p. 178]. We deduce part (2). Part (3) follows from (5.2). The last part of the lemma is straightforward.

We will normalize the measures on $\mathrm{Q}^{x}, x \in \mathrm{X}_{\mathrm{L}}$ by the recipe of Section 1.2. To that end, we need to specify the gauge form $\boldsymbol{\omega}_{\mathbf{Q}^{x}}$ and the scalar $\lambda_{\mathbf{Q}^{x}}$. First, let $\boldsymbol{\omega}_{\mathbf{L}^{x}}=\boldsymbol{\omega}_{\mathbf{M}_{\boldsymbol{k}}} \wedge$ $\boldsymbol{\omega}_{\mathbf{G}_{\boldsymbol{m}}^{y}}$ be the gauge form on $\mathbf{L}^{\boldsymbol{x}}$ defined via the isomorphism (5.3), and let $\boldsymbol{\lambda}_{\mathbf{L}^{x}}=\boldsymbol{\lambda}_{\mathbf{M}_{\kappa}} \boldsymbol{\lambda}_{\mathbf{G}_{m}^{y}}$ where $y=p_{m}(x)$. For any $x \in \mathrm{X}_{\mathrm{L}}$ we take $\boldsymbol{\omega}_{x+\mathbf{Y}}=\prod d x_{i, j}$ where the product is over all non-zero coordinates of $\mathbf{Y}$ such that $i \leq j$ (where the coordinates are in $\mathrm{E}$ if $i<j$ and in $\mathrm{F}$ if $i=j$ ) and take $\boldsymbol{\omega}_{\mathbf{V}^{x}}$ which matches $\boldsymbol{\omega}_{\mathbf{V}}$ and $\boldsymbol{\omega}_{x+\mathbf{Y}}$. Finally, applying Lemma 5.1 (2), we define $\boldsymbol{\omega}_{\mathbf{Q}^{x}}$ to be matching with $\boldsymbol{\omega}_{\mathbf{L}^{x}}$ and $\boldsymbol{\omega}_{\mathbf{V}^{x}}$, and take $\boldsymbol{\lambda}_{\mathbf{Q}^{x}}=\boldsymbol{\lambda}_{\mathbf{L}^{x}}$.

We also define a gauge form on $\mathbf{X}_{\mathbf{L}}$ by $\boldsymbol{\omega}_{\mathbf{X}_{\mathbf{L}}}=\boldsymbol{\omega}_{\mathbf{M}_{k}} \wedge \boldsymbol{\omega}_{\mathbf{X}_{m}}$ via the isomorphism (5.1) and the scalar $\lambda_{\mathbf{x}_{\mathbf{L}}}=\boldsymbol{\lambda}_{\mathbf{M}_{\kappa}} \boldsymbol{\lambda}_{\mathbf{x}_{\boldsymbol{m}}}$. The isomorphism $\mathrm{X}_{\mathrm{L}} \simeq \coprod_{x \in \mathrm{X}_{\mathrm{L}} / \mathrm{L}} \mathrm{L}^{x} \backslash \mathrm{L}$ is compatible with measures.

For a Levi subgroup $\mathrm{M}$ of $\mathrm{G}$, an $\mathrm{M}$-invariant subset $\mathrm{C}$ of $\mathrm{X}$ and $\sigma \in \mathcal{R}(\mathrm{M})$, let

$$
\mathcal{E}_{\mathrm{M}}\left(\mathrm{C}, \sigma^{*}\right)=\left\{\beta: \mathrm{C} \rightarrow \sigma^{*}: \beta_{x \bullet g}=\beta_{x} \circ \sigma(g) \text { for all } x \in \mathrm{C} \text { and } g \in \mathrm{M}\right\} .
$$

Any $w \in \mathrm{W}$ such that $w \mathrm{M} w^{-1}=\mathrm{M}$ acts on $\mathfrak{a}_{\mathrm{M}}^{*}$ and we define

$$
\left(\mathfrak{a}_{\mathrm{M}}^{*}\right)^{w}=\left\{\lambda \in \mathfrak{a}_{\mathrm{M}}^{*}: w \lambda=\lambda\right\} .
$$

We have

$$
\left(\mathfrak{a}_{\mathrm{L}}^{*}\right)^{w_{*}}= \begin{cases}\left\{(\mu, \overleftarrow{\mu}): \mu \in \mathfrak{a}_{\mathrm{M}_{\kappa}}^{*}\right\} & m=0 \\ \left\{(\mu, z, \overleftarrow{\mu}): \mu \in \mathfrak{a}_{\mathrm{M}_{\kappa}}^{*}, z \in \mathbf{R}\right\} & \text { otherwise }\end{cases}
$$

It follows from (5.3) and Lemma 5.1(2) that for $x \in \mathrm{X}_{\mathrm{L}}$ we have

$$
\langle\lambda, \mathrm{H}(g)\rangle=0, \quad g \in \mathrm{Q}^{x}, \lambda \in\left(\mathfrak{a}_{\mathrm{L}}^{*}\right)_{\mathbf{C}}^{w_{*}} .
$$

For $\varrho \in \mathcal{R}(\mathrm{L}), \beta \in \mathcal{E}_{\mathrm{L}}\left(\mathrm{X}_{\mathrm{L}}, \varrho^{*}\right), x \in \mathrm{X}$ and $\lambda \in\left(\mathfrak{a}_{\mathrm{L}}^{*}\right)_{\mathbf{C}}^{w_{*}}$ define the closed period $\mathrm{Z}(x, \beta, \lambda)=\mathrm{Z}_{\mathrm{L}}^{\mathrm{G}}(x, \beta, \lambda)$ as follows. If $x \bullet \mathrm{G} \cap \mathrm{X}_{\mathrm{L}}=\emptyset$ we set $\mathrm{Z}(x, \beta, \lambda)=0$. Otherwise, there exist $y \in \mathrm{X}_{\mathrm{L}}$ and $\iota_{y}^{x} \in \mathrm{G}$ such that $x=y \bullet \iota_{y}^{x}$ and we define

$$
\mathrm{Z}(\varphi: x, \beta, \lambda)=e^{\frac{1}{2}\langle\lambda, \mathrm{H}(y)\rangle} \int_{\mathrm{Q}^{y} \backslash \mathrm{G}^{\nu}} \beta_{y}\left(\varphi_{\lambda}\left(h \iota_{y}^{x}\right)\right) d h, \quad \varphi \in \mathrm{I}_{\mathrm{L}}^{\mathrm{G}}(\varrho) .
$$


In particular, if $m=0$ then

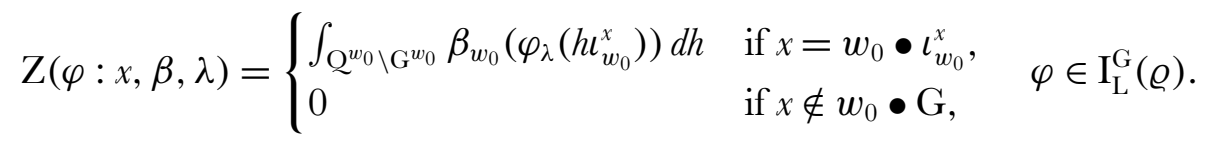

Lemma 5.2. - The expression (5.5) is well-defined and holomorphic in $\lambda \in\left(\mathfrak{a}_{\mathrm{L}}^{*}\right)_{\mathbf{C}}^{w_{*}}$. Furthermore, the map $x \mapsto \mathrm{Z}(x, \beta, \lambda)$ is an element of $\mathcal{E}_{\mathrm{G}}\left(\mathrm{X}, \mathrm{I}_{\mathrm{L}}^{\mathrm{G}}(\varrho, \lambda)^{*}\right)$ that we denote by $\mathrm{Z}(\beta, \lambda)$.

Moreover, for any $\lambda \in\left(\mathfrak{a}_{\mathrm{L}}^{*}\right)_{\mathbf{C}}^{w_{*}}, \mathrm{Z}(x, \beta, \lambda) \not \equiv 0$ if and only if there exists $y \in x \bullet \mathrm{G} \cap \mathrm{X}_{\mathrm{L}}$ such that $\beta_{y} \not \equiv 0$.

In particular, for $m=0, \mathrm{Z}(x, \beta, \lambda) \not \equiv 0$ if and only if $x \in w_{0} \bullet \mathrm{G}$ (i.e., $\mathrm{G}^{x}$ is quasi-split) and $\beta \not \equiv 0$.

Proof. - Recall that by [LR03, Proposition 4.3.2] we have $\left.\delta_{\mathrm{Q}^{\frac{1}{2}}}\right|_{\mathrm{Q}^{y}}=\delta_{\mathrm{Q}^{\nu}}$. Therefore, it follows from the equivariance property of $\beta,(\mathbf{5 . 4})$ and the fact that $\mathrm{Q}^{y}$ is a parabolic subgroup of $\mathrm{G}^{y}$ (Lemma 5.1(2)) that the integral in (5.5) is well-defined and holomorphic in $\lambda$.

If $x=y \bullet \iota_{2}$ for $\iota_{2} \in \mathrm{G}$ then $\iota_{y}^{x} \iota_{2}^{-1} \in \mathrm{G}^{y}$ and the change of variables $h \mapsto h\left(\iota_{y}^{x} \iota_{2}^{-1}\right)^{-1}$ shows that (5.5) is independent of the choice of $\iota_{y}^{x}$. If $y^{\prime} \in \mathrm{X}_{\mathrm{L}}$ is such that $y \bullet \mathrm{G}=y^{\prime} \bullet \mathrm{G}$ then it follows from Lemma 5.1(1) that there exists $\ell \in \mathrm{L}$ such that $y^{\prime}=y \bullet \ell$ and we may therefore choose $\iota_{y^{\prime}}^{x}=\ell^{-1} \iota_{y}^{x}$. Since

$$
e^{\left\langle\rho_{\mathrm{L}}, \mathrm{H}(\ell)\right\rangle} e^{\frac{1}{2}\left\langle\lambda, \mathrm{H}\left(y^{\prime}\right)\right\rangle} \beta_{y^{\prime}}\left(\varphi_{\lambda}\left(\ell^{-1} g\right)\right)=e^{\frac{1}{2}\langle\lambda, \mathrm{H}(y)\rangle} \beta_{y}\left(\varphi_{\lambda}(g)\right), \quad g \in \mathrm{G}
$$

and the change of variable $h \mapsto \ell h \ell^{-1}$ transforms our measure on $Q^{y} \backslash \mathrm{G}^{y}$ to $e^{-\left\langle\rho_{\mathrm{L}}, \mathrm{H}(\ell)\right\rangle}$ times our measure on $Q^{y^{\prime}} \backslash \mathrm{G}^{y^{\prime}}$, this change of variables shows that (5.5) is independent of the choice of $y$.

The equivariance property is trivial if $x \bullet \mathrm{G} \cap \mathrm{X}_{\mathrm{L}}=\emptyset$. Otherwise, it follows by choosing $\iota_{y}^{x} g=\iota_{y}^{x} g$ for $g \in \mathrm{G}$.

For the non-vanishing criterion, the 'only if' part is trivial. For the 'if' part, we may suppose that $x=y \in \mathrm{X}_{\mathrm{L}}$ and $\beta_{x} \not \equiv 0$. Let $v \in \varrho$ be such that $\beta_{x}(v) \neq 0$. By Lemma 5.1(2), we may replace the integration in (5.5) over $Q^{y} \backslash \mathrm{G}^{y}$ by integration over $\left({ }^{t} \mathrm{~V}\right)^{\nu}$. Fix $f \in \mathrm{C}_{c}^{\infty}\left({ }^{t} \mathrm{~V}\right) \geq 0$ with $f(e)>0$ and let $\varphi \in \mathrm{I}_{\mathrm{L}}^{\mathrm{G}}(\varrho)$ be the section supported in $\mathrm{Q}^{t} \mathrm{~V}$ such that $\varphi_{\lambda}(u)=f(u) v$ for $u \in{ }^{t} \mathrm{~V}$. Then up to a positive scalar, $\mathrm{Z}(\varphi: x, \beta, \lambda)$ is given by $\beta_{x}(v) \int_{\left({ }^{\prime} \mathrm{V}\right)^{x}} f(u) d u$ and hence it is non-zero.

The map

$$
\mathcal{S}(\mathrm{X}) \rightarrow \mathcal{S}\left(\mathrm{X}_{\mathrm{L}}\right)
$$

which takes $\Phi$ to

$$
\Phi_{\mathrm{V}}(y)=\int_{\mathrm{V}^{y} \backslash \mathrm{V}} \Phi(y \bullet v) d v, \quad y \in \mathrm{X}_{\mathrm{L}}
$$


induces an L-equivariant map

$$
r_{\mathrm{L}}(\mathcal{S}(\mathrm{X})) \rightarrow \mathcal{S}\left(\mathrm{X}_{\mathrm{L}}\right)
$$

On the other hand, we may identify

$$
\mathcal{E}_{\mathrm{L}}\left(\mathrm{X}_{\mathrm{L}}, \varrho^{*}\right) \simeq \operatorname{Hom}_{\mathrm{L}}\left(\mathcal{S}\left(\mathrm{X}_{\mathrm{L}}\right), \varrho^{\vee}\right)
$$

and

$$
\mathcal{E}_{\mathrm{G}}\left(\mathrm{X}, \mathrm{I}_{\mathrm{L}}^{\mathrm{G}}(\varrho, \lambda)^{*}\right) \simeq \operatorname{Hom}_{\mathrm{G}}\left(\mathcal{S}(\mathrm{X}), \mathrm{I}(\varrho, \lambda)^{\vee}\right) \simeq \operatorname{Hom}_{\mathrm{L}}\left(r_{\mathrm{L}}(\mathcal{S}(\mathrm{X})), \varrho^{\vee}[-\lambda]\right)
$$

(by Frobenius reciprocity, cf. the discussion before Lemma 4.3). For $\beta \in \mathcal{E}_{\mathrm{L}}\left(\mathrm{X}_{\mathrm{L}}, \varrho^{*}\right)$ let $\beta[\lambda]=\left(y \mapsto e^{\frac{1}{2}\langle\lambda, \mathrm{H}(y)\rangle} \beta_{y}\right) \in \mathcal{E}_{\mathrm{L}}\left(\mathrm{X}_{\mathrm{L}}, \varrho[\lambda]^{*}\right)$.

Lemma 5.3. - Under the above identifications, the map $\beta[\lambda] \mapsto \mathrm{Z}(\beta, \lambda)$ becomes the map

$$
\operatorname{Hom}_{\mathrm{L}}\left(\mathcal{S}\left(\mathrm{X}_{\mathrm{L}}\right), \varrho^{\vee}[-\lambda]\right) \rightarrow \operatorname{Hom}_{\mathrm{L}}\left(r_{\mathrm{L}}(\mathcal{S}(\mathrm{X})), \varrho^{\vee}[-\lambda]\right)
$$

obtained by composition with the map $(\mathbf{5 . 8})$.

Proof. - If $\beta \in \mathcal{E}_{\mathrm{L}}\left(\mathrm{X}_{\mathrm{L}}, \varrho^{*}\right)$ is such that $\beta[\lambda]$ corresponds to $\mathrm{A} \in$ $\operatorname{Hom}_{\mathrm{L}}\left(\mathcal{S}\left(\mathrm{X}_{\mathrm{L}}\right), \varrho^{\vee}[-\lambda]\right)$ then

$$
\mathrm{A}(\Psi)(v)=\int_{\mathrm{X}_{\mathrm{L}}} \Psi(x) e^{\frac{1}{2}\langle\lambda, \mathrm{H}(x)\rangle} \beta_{x}(v) d x, \quad \Psi \in \mathcal{S}\left(\mathrm{X}_{\mathrm{L}}\right), v \in \varrho
$$

and we need to show that for $\Phi \in \mathcal{S}(\mathrm{X})$ and $\varphi \in \mathrm{I}(\varrho)$ we have

$$
\int_{\mathrm{X}} \Phi(x) \mathrm{Z}(\varphi: x, \beta, \lambda) d x=\int_{\mathrm{Q} \backslash \mathrm{G}} \mathrm{A}\left((\mathrm{R}(g) \Phi)_{\mathrm{V}}\right)\left(\varphi_{\lambda}(g)\right) d g .
$$

From the definition of $\mathrm{Z}$ we get

$$
\begin{aligned}
\int_{\mathrm{X}} & \Phi(x) \mathrm{Z}(\varphi: x, \beta, \lambda) d x \\
= & \sum_{y \in \mathrm{X}_{\mathrm{L}} / \mathrm{L}} e^{\frac{1}{2}\langle\lambda, \mathrm{H}(y)\rangle} \int_{\mathrm{G}^{y} \backslash \mathrm{G}} \Phi(y \bullet g) \int_{\mathrm{Q}^{y} \backslash \mathrm{G}^{y}} \beta_{y}\left(\varphi_{\lambda}(h g)\right) d h d g \\
= & \sum_{y \in \mathrm{X}_{\mathrm{L}} / \mathrm{L}} e^{\frac{1}{2}\langle\lambda, \mathrm{H}(y)\rangle} \int_{\mathrm{Q}^{y \mathrm{G}}} \Phi(y \bullet g) \beta_{y}\left(\varphi_{\lambda}(g)\right) d g \\
= & \sum_{y \in \mathrm{X}_{\mathrm{L}} / \mathrm{L}} e^{\frac{1}{2}\langle\lambda, \mathrm{H}(y)\rangle} \int_{\mathrm{Q} \backslash \mathrm{G}} \int_{\mathrm{Q}^{y} \backslash \mathrm{Q}} \Phi(y \bullet q g) \beta_{y}\left(\varphi_{\lambda}(q g)\right) \delta_{\mathrm{Q}}(q)^{-1} d q d g
\end{aligned}
$$




$$
\begin{aligned}
& =\sum_{y \in \mathrm{X}_{\mathrm{L}} / \mathrm{L}} e^{\frac{1}{2}\langle\lambda, \mathrm{H}(y)\rangle} \int_{\mathrm{Q} \backslash \mathrm{G}} \int_{\mathrm{L}^{y} \backslash \mathrm{L}} \int_{\mathrm{V}^{y} \backslash \mathrm{V}} \Phi(y \bullet v l g) \delta_{\mathrm{Q}}(l)^{-1} \beta_{y}\left(\varphi_{\lambda}(l g)\right) d v d l d g \\
& =\sum_{y \in \mathrm{X}_{\mathrm{L}} / \mathrm{L}} e^{\frac{1}{2}\langle\lambda, \mathrm{H}(y)\rangle} \int_{\mathrm{Q}_{\backslash \mathrm{G}}} \int_{\mathrm{L}^{y} \backslash \mathrm{L}}(\mathrm{R}(g) \Phi)_{\mathrm{V}}(y \bullet l) \beta_{y}\left(\varphi_{\lambda}(l g)\right) \delta_{\mathrm{Q}}(l)^{-\frac{1}{2}} d l d g \\
& \stackrel{(\mathbf{5 . 7})}{=} \int_{\mathrm{Q} \backslash \mathrm{G}} \sum_{y \in \mathrm{X}_{\mathrm{L}} / \mathrm{L}} \int_{\mathrm{L}^{y} \backslash \mathrm{L}}(\mathrm{R}(g) \Phi)_{\mathrm{V}}(y \bullet l) e^{\frac{1}{2}\langle\lambda, \mathrm{H}(y \bullet l)\rangle} \beta_{y \bullet l}\left(\varphi_{\lambda}(g)\right) d y d g \\
& =\int_{\mathrm{Q} \backslash \mathrm{G}} \int_{\mathrm{X}_{\mathrm{L}}}(\mathrm{R}(g) \Phi)_{\mathrm{V}}(x) e^{\frac{1}{2}\langle\lambda, \mathrm{H}(x)\rangle} \beta_{x}\left(\varphi_{\lambda}(g)\right) d x d g \\
& =\int_{\mathrm{Q} \backslash \mathrm{G}} \mathrm{A}\left((\mathrm{R}(g) \Phi)_{\mathrm{V}}\right)\left(\varphi_{\lambda}(g)\right) d g .
\end{aligned}
$$

The lemma follows.

5.2. Further analysis. - Next, we would like to connect the closed periods above to the open periods defined in Section 4 and to reduce the study of the closed periods to the even symmetric case $(m=0)$.

Assume that $m>0$. Let $\mathrm{M}=\mathrm{M}_{(k+m, k)} \supset \mathrm{L}$ and introduce the pair of Levi subgroups $\tilde{\mathrm{L}} \subseteq \tilde{\mathrm{M}}$ where $\tilde{\mathrm{L}}=\mathrm{M}_{(m, \kappa, \overleftarrow{\kappa})}$ and $\tilde{\mathrm{M}}=\mathrm{M}_{(m, 2 k)}$. Let $\mathrm{P}=\mathrm{MU}, \tilde{\mathrm{P}}=\tilde{\mathrm{M}} \tilde{\mathrm{U}}$ and $\tilde{\mathrm{Q}}=\tilde{\mathrm{L}} \tilde{\mathrm{V}}$ be the corresponding standard parabolic subgroups. Let also $w=w_{(m, k, k)}^{(m+k, k)} \in \mathbf{W}(\tilde{\mathrm{L}})$, so that $\tilde{\mathrm{L}}=w^{-1} \mathrm{~L} w$, and set $\widetilde{w_{*}}=w^{-1} w_{*} w=\operatorname{diag}\left(1_{m}, w_{0}^{\mathrm{G}_{2 k}}\right)$.

Let $\mathrm{X}_{\tilde{\mathrm{L}}}^{\tilde{\mathrm{M}}}=\widetilde{w_{*}} \tilde{\mathrm{L}} \cap \mathrm{X}^{\tilde{\mathrm{M}}}=\widetilde{w_{*}} \tilde{\mathrm{L}} \cap \mathrm{X}$ and note that $y \mapsto y \bullet w: \mathrm{X}_{\mathrm{L}} \rightarrow \mathrm{X}_{\tilde{\mathrm{L}}}^{\tilde{\mathrm{M}}}$ is an isomorphism. Accordingly, for any $\varrho \in \mathcal{R}(\tilde{\mathrm{L}})$ the map $\beta \mapsto w \beta$, where $(w \beta)_{y}=\beta_{y \bullet w}$ for $y \in \mathrm{X}_{\mathrm{L}}$ defines an isomorphism $\mathcal{E}_{\tilde{\mathrm{L}}}\left(\mathrm{X}_{\tilde{\mathrm{L}}}^{\tilde{\mathrm{M}}}, \varrho^{*}\right) \rightarrow \mathcal{E}_{\mathrm{L}}\left(\mathrm{X}_{\mathrm{L}},(w \varrho)^{*}\right)$.

Note that

$$
\mathrm{X}_{\tilde{\mathrm{L}}}^{\tilde{\mathrm{M}}}=\left\{\operatorname{diag}\left(x_{1}, x_{2}\right): x_{1} \in \mathrm{X}_{m}, x_{2} \in \mathrm{X}_{\mathrm{M}_{(\kappa, \overleftarrow{\kappa})}}^{\mathrm{G}_{2 k}}\right\}
$$

and

$$
\left(\mathfrak{a}_{\tilde{\mathrm{L}}}^{*}\right)_{\mathbf{C}}^{\widetilde{w_{*}}}=\left\{(z, \mu, \overleftarrow{\mu}): z \in \mathbf{C}, \mu \in \mathfrak{a}_{\mathrm{M}_{\kappa}, \mathbf{G}}^{*}\right\}=w^{-1}\left(\mathfrak{a}_{\mathrm{L}}^{*}\right)_{\mathbf{G}}^{w_{*}}
$$

For any $x=\operatorname{diag}\left(x_{1}, x_{2}\right) \in \mathrm{X}_{\tilde{\mathrm{L}}}^{\tilde{\tilde{I}}}$ we have $\tilde{\mathrm{U}}^{x}=1$ and therefore, $\tilde{\mathrm{Q}}^{x} \subseteq \tilde{\mathrm{P}}^{x}=\tilde{\mathrm{M}}^{x} \tilde{\mathrm{U}}^{x}=\tilde{\mathrm{M}}^{x}$. In fact,

$$
\tilde{\mathrm{Q}}^{x}=\left\{\operatorname{diag}(g, p): g \in \mathrm{G}_{m}^{x_{1}}, p \in \mathrm{P}_{(\kappa, \overleftarrow{\kappa})}^{x_{2}}\right\} \simeq \mathrm{G}_{m}^{x_{1}} \times \mathrm{P}_{(\kappa, \overleftarrow{\kappa})}^{x_{2}}
$$

We endow $\mathrm{P}_{(\kappa, \overleftarrow{\kappa})}^{x_{2}}$ with a Haar measure as in the discussion following Lemma 5.1. This gives rise to a Haar measure on $\tilde{Q}^{x}$. 
For $\varrho \in \mathcal{R}(\tilde{\mathrm{L}}), \beta \in \mathcal{E}_{\tilde{\mathrm{L}}}\left(\mathrm{X}_{\tilde{\mathrm{L}}}^{\tilde{\mathrm{M}}}, \varrho^{*}\right)$ and $\lambda \in\left(\mathfrak{a}_{\tilde{\mathrm{L}}}^{*}\right)_{\mathbf{\mathbf { c }}}^{\widetilde{w_{*}}}$, let $\mathrm{Z}^{\tilde{\mathrm{M}}}(\beta, \lambda)$ be defined by

$$
\mathrm{Z}^{\tilde{\mathrm{M}}}(\varphi: x, \beta, \lambda)= \begin{cases}e^{\frac{1}{2}\langle\lambda, \mathrm{H}(y)\rangle} \int_{\tilde{\mathrm{Q}} \backslash \tilde{\mathrm{M}}^{y}} \beta_{y}\left(\varphi_{\lambda}\left(h \iota_{y}^{x}\right)\right) d h & x=y \bullet \iota_{y}^{x}, y \in \mathrm{X}_{\tilde{\mathrm{L}}}^{\tilde{\mathrm{M}}}, \iota_{y}^{x} \in \tilde{\mathrm{M}}, \\ 0 & x \notin \mathrm{X}_{\tilde{\mathrm{L}}}^{\tilde{\mathrm{M}}} \bullet \tilde{\mathrm{M}},\end{cases}
$$

for $\varphi \in \mathrm{I}_{\tilde{\mathrm{L}}}^{\tilde{\mathrm{M}}}(\varrho)$ and $x \in \mathrm{X}^{\tilde{\mathrm{M}}}$. Note that as in Lemma 5.2 (for the special case $m=0$ ), $\mathrm{Z}^{\tilde{\mathrm{M}}}(\beta, \lambda)$ is a well defined element of $\mathcal{E}_{\tilde{\mathrm{M}}}\left(\mathrm{X}^{\tilde{\mathrm{M}}}, \mathrm{I}_{\tilde{\mathrm{L}}}^{\tilde{\mathrm{M}}}(\varrho, \lambda)\right)$, entire in $\lambda$ and

(5.10) $\quad \mathrm{Z}^{\tilde{\mathrm{M}}}(x, \beta, \lambda) \not \equiv 0 \quad$ if and only if there exists $\quad y \in \mathrm{X}_{\tilde{\mathrm{L}}}^{\tilde{\mathrm{M}}} \cap x \bullet \tilde{\mathrm{M}}$ such that $\beta_{y} \not \equiv 0$.

Proposition 5.4. - For any $\varrho \in \mathcal{R}(\tilde{\mathrm{L}})$ and $\beta \in \mathcal{E}_{\tilde{\mathrm{L}}}\left(\mathrm{X}_{\tilde{\mathrm{L}}}^{\tilde{\mathrm{M}}}, \varrho^{*}\right)$ we have

$$
\mathrm{J}_{\tilde{\mathrm{M}}}\left(\mathrm{Z}^{\tilde{\mathrm{M}}}(\beta, \lambda), 0\right) \circ \Gamma_{\tilde{\mathrm{L}}, \tilde{\mathrm{M}}}(\varrho, \lambda)=\eta(-1)^{k} \mathrm{Z}(w \beta, w \lambda) \circ \mathrm{M}(w, \varrho, \lambda)
$$

as an identity of meromorphic functions in $\lambda \in\left(\mathfrak{a}_{\tilde{\mathbf{L}}}^{*}\right)_{\mathbf{G}}^{\widetilde{w_{*}}}$.

We first need some more notation. Let $\mathrm{L}_{1}=\mathrm{M}_{(k, m, k)}$ and $\tilde{\mathrm{L}}_{1}=\mathrm{M}_{(m, k, k)}=$ $w^{-1} \mathrm{~L}_{1} w=\mathrm{M} \cap \tilde{\mathrm{M}}$, so that $\mathrm{L} \subseteq \mathrm{L}_{1} \subseteq \mathrm{M}, \tilde{\mathrm{L}} \subseteq \tilde{\mathrm{L}}_{1} \subseteq \tilde{\mathrm{M}}$ and $w=w_{\tilde{\mathrm{L}}_{1}}^{\mathrm{M}}$. Let $\mathrm{Q}_{1}=\mathrm{L}_{1} \mathrm{~V}_{1}$ be the corresponding standard parabolic subgroups of $\mathrm{G}$. Also, let $\mathrm{R}$ be the non-standard parabolic subgroup $w^{-1} \mathrm{Q} w \subseteq \mathrm{P}$ and let $\tilde{\mathrm{V}}_{1}=w^{-1} \mathrm{~V}_{1} w$. Explicitly,

$$
\begin{aligned}
& \mathrm{R}=\left(\begin{array}{ccc}
\mathrm{G}_{m} & 0 & * \\
* & \mathrm{P}_{\kappa} & * \\
0 & 0 & \mathrm{P}_{\overleftarrow{\kappa}}
\end{array}\right) ; \quad \tilde{\mathrm{Q}} \cap \tilde{\mathrm{L}}_{1}=\left(\begin{array}{ccc}
\mathrm{G}_{m} & 0 & 0 \\
0 & \mathrm{P}_{\kappa} & 0 \\
0 & 0 & \mathrm{P}_{\overleftarrow{\kappa}}
\end{array}\right) ; \\
& \tilde{\mathrm{V}}_{1}=\left(\begin{array}{ccc}
\mathrm{I}_{m} & 0 & * \\
* & \mathrm{I}_{k} & * \\
0 & 0 & \mathrm{I}_{k}
\end{array}\right) ; \quad \mathrm{U} \cap \tilde{\mathrm{M}}=\left(\begin{array}{ccc}
\mathrm{I}_{m} & 0 & 0 \\
0 & \mathrm{I}_{k} & * \\
0 & 0 & \mathrm{I}_{k}
\end{array}\right) \text {. }
\end{aligned}
$$

Note that $\mathrm{R}=\left(\tilde{\mathrm{Q}} \cap \tilde{\mathrm{L}}_{1}\right) \ltimes \tilde{\mathrm{V}}_{1}$ and $\tilde{\mathrm{Q}}=\left(\tilde{\mathrm{Q}} \cap \tilde{\mathrm{L}}_{1}\right) \ltimes(\mathrm{U} \cap \tilde{\mathrm{M}}) \tilde{\mathrm{U}}$. The following result follows by straightforward verification.

Lemma 5.5. - Let $y \in \mathrm{X}_{\mathrm{L}}$ and $x=y \bullet w \in \mathrm{X}_{\tilde{\mathrm{L}}}^{\tilde{\mathrm{M}}}$. Then

$$
\mathrm{R}^{x}=\left(\tilde{\mathrm{Q}} \cap \tilde{\mathrm{L}}_{1}\right)^{x} \ltimes \tilde{\mathrm{V}}_{1}^{x} \supset \tilde{\mathrm{Q}}^{x}=\left(\tilde{\mathrm{Q}} \cap \tilde{\mathrm{L}}_{1}\right)^{x} \ltimes(\mathrm{U} \cap \tilde{\mathrm{M}})^{x}
$$

so that

(5.12)

$$
\tilde{Q}^{x} \backslash \mathrm{R}^{x} \simeq(\mathrm{U} \cap \tilde{\mathrm{M}})^{x} \backslash \tilde{\mathrm{V}}_{1}^{x} .
$$

Moreover, the projection of $\mathrm{P}$ onto $\mathrm{M}$ induces an isomorphism

$$
(\mathrm{U} \cap \tilde{\mathrm{M}})^{x} \backslash \tilde{\mathrm{V}}_{1}^{x} \rightarrow \mathrm{M} \cap \tilde{\mathrm{V}}_{1}=w^{-1}\left(\mathrm{M} \cap \mathrm{V}_{1}\right) w
$$


Transfer the measure on $\mathrm{Q}^{y}$ (defined after Lemma 5.1) to $\mathrm{R}^{x}$ via the relation $\mathrm{R}^{x}=w^{-1} \mathrm{Q}^{y} w$. Transfer the quotient measure on $\tilde{Q}^{x} \backslash \mathrm{R}^{x}$ to $(\mathrm{U} \cap \tilde{\mathrm{M}})^{x} \backslash \tilde{\mathrm{V}}_{1}^{x}$ via $(\mathbf{5 . 1 2})$. Then under the isomorphism $(\mathbf{5 . 1 3})$, the measure on $(\mathrm{U} \cap \tilde{\mathrm{M}})^{x} \backslash \tilde{\mathrm{V}}_{1}^{x}$ becomes $e^{-\frac{1}{2}\left\langle\rho_{\tilde{\mathrm{M}}}, \mathrm{H}(x)\right\rangle}$ times the measure on $w^{-1}\left(\mathrm{M} \cap \mathrm{V}_{1}\right) w$ (which is the transfer of our measure on $\mathrm{M} \cap \mathrm{V}_{1}$ via $\left.m \mapsto w^{-1} m w\right)$.

Proof of Proposition 5.4. - Fix $x \in \mathrm{X}$ and write for simplicity

$$
\mathrm{J}=\mathrm{J}_{\tilde{\mathrm{M}}}\left(x, \mathrm{Z}^{\tilde{\mathrm{M}}}(\beta, \lambda), 0\right) \in \operatorname{Hom}_{\mathrm{G}^{x}}\left(\mathrm{I}_{\tilde{\mathrm{M}}}\left(\mathrm{I}_{\tilde{\mathrm{L}}}^{\tilde{\mathrm{M}}}(\varrho, \lambda), 0\right), \mathbf{G}\right) .
$$

Note that if $\beta_{y} \equiv 0$ for all $y \in x \bullet \mathrm{G} \cap \mathrm{X}_{\tilde{\mathrm{L}}}^{\tilde{\mathrm{M}}}$ then $(w \beta)_{y} \equiv 0$ for all $y \in x \bullet \mathrm{G} \cap \mathrm{L}$ and both sides of (5.11) vanish (by (5.10) and Lemma 5.2). Assume therefore that there exists $y \in x \bullet \mathrm{G} \cap \mathrm{X}_{\tilde{\mathrm{L}}}^{\tilde{\mathrm{M}}}$ such that $\beta_{y} \not \equiv 0$. By meromorphic continuation, it suffices to consider $\lambda$ such that $\operatorname{Re} \lambda_{\tilde{M}} \in\left(\mathfrak{a}_{\tilde{\mathrm{M}}}^{*}\right)_{+}$is sufficiently regular. Note that $J$ is defined in (4.2) as the sum over $(x \bullet \mathrm{G} \cap \tilde{\mathrm{M}}) / \tilde{\mathrm{M}}$ of certain integrals and that $y \bullet \tilde{\mathrm{M}}$ is the only $\tilde{\mathrm{M}}$-orbit in $x \bullet \mathrm{G} \cap \tilde{\mathrm{M}}$ that has non-empty intersection with $\mathrm{X}_{\tilde{\mathrm{L}}}^{\tilde{\mathrm{M}}}$. By $(\mathbf{5 . 1 0})$, it follows that J equals the summand parameterized by $y \bullet \tilde{M}$. Without loss of generality, we may therefore assume that $x \in \mathrm{X}_{\tilde{\mathrm{L}}}^{\tilde{\mathrm{M}}}$. Note that in this case $v_{\tilde{\mathrm{M}}}(x)=\eta(-1)^{k}$. Let $y=x \bullet w^{-1} \in \mathrm{X}_{\mathrm{L}}$ be as in Lemma 5.5. Fix $\varphi \in \mathrm{I}(\varrho)$ and let $\xi=\Gamma_{\tilde{\mathrm{L}}, \tilde{\mathrm{M}}}(\varrho, \lambda) \varphi$. We have

$$
\begin{aligned}
\mathrm{J}(\xi) & =\eta(-1)^{k} e^{\frac{1}{2}\left\langle\rho_{\tilde{\mathrm{M}}}, \mathrm{H}(x)\right\rangle} \int_{\tilde{\mathrm{M}}^{x} \backslash \mathrm{G}^{x}} \mathrm{Z}^{\tilde{\mathrm{M}}}(\xi(g): x, \beta, \lambda) d g \\
& =\eta(-1)^{k} e^{\frac{1}{2}\left\langle\rho_{\tilde{\mathrm{M}}}+\lambda, \mathrm{H}(x)\right\rangle} \int_{\tilde{\mathrm{M}}^{x} \backslash \mathrm{G}^{x}} \int_{\tilde{\mathrm{Q}}^{x} \backslash \tilde{\mathrm{M}}^{x}} \beta_{x}\left(\xi_{\lambda}(g)(l)\right) d l d g
\end{aligned}
$$

where the integral is absolutely convergent (by our assumption on $\lambda$ ). By $(\mathbf{1 . 1})$ and the fact that $\mathrm{H}\left(\tilde{\mathrm{M}}^{x}\right)_{\tilde{\mathrm{M}}}=0$ we get $\eta(-1)^{k} e^{\frac{1}{2}\langle\lambda, \mathrm{H}(x)\rangle}$ times

$$
e^{\frac{1}{2}\left\langle\rho_{\tilde{\mathrm{M}}}, \mathrm{H}(x)\right\rangle} \int_{\tilde{\mathrm{M}}^{x} \backslash \mathrm{G}^{x}} \int_{\tilde{\mathrm{Q}}^{x} \backslash \tilde{\mathrm{M}}^{x}} \beta_{x}\left(\varphi_{\lambda}(l g)\right) d l d g=e^{\frac{1}{2}\left\langle\rho_{\tilde{\mathrm{M}}}, \mathrm{H}(x)\right\rangle} \int_{\tilde{\mathbf{Q}}^{x} \backslash \mathrm{G}^{x}} \beta_{x}\left(\varphi_{\lambda}(g)\right) d g .
$$

By Lemma 5.5 this becomes

$$
\begin{aligned}
& e^{\frac{1}{2}\left\langle\rho_{\tilde{\mathrm{M}}}, \mathrm{H}(x)\right\rangle} \int_{\mathrm{R}^{x} \backslash \mathrm{G}^{x}} \int_{\tilde{\mathrm{Q}}^{x} \backslash \mathrm{R}^{x}} \delta_{\mathrm{R}^{x}}^{-1}(r) \beta_{x}\left(\varphi_{\lambda}(r g)\right) d r d g \\
& =e^{\frac{1}{2}\left\langle\rho_{\tilde{\mathrm{M}}}, \mathrm{H}(x)\right\rangle} \int_{\mathrm{R}^{x} \backslash \mathrm{G}^{x}} \int_{(\mathrm{U} \cap \tilde{\mathrm{M}})^{x} \backslash \tilde{\mathrm{V}}_{1}^{x}} \beta_{x}\left(\varphi_{\lambda}(u g)\right) d u d g \\
& =\int_{\mathrm{R}^{x} \backslash \mathrm{G}^{x}} \int_{\mathrm{M} \cap \mathrm{V}_{1}} \beta_{x}\left(\varphi_{\lambda}\left(w^{-1} u w g\right)\right) d u d g \\
& =\int_{\mathrm{R}^{x} \backslash \mathrm{G}^{x}} \beta_{x}\left((\mathrm{M}(w, \varrho, \lambda) \varphi)_{w \lambda}(w g)\right) d g .
\end{aligned}
$$


By the change of variable $g \mapsto w^{-1} g w$, we get

$$
\int_{Q^{y} \backslash \mathrm{G}^{y}}(w \beta)_{y}\left((\mathrm{M}(w, \varrho, \lambda) \varphi)_{w \lambda}(g w)\right) d g .
$$

Altogether, we get $\mathrm{J}(\xi)=\eta(-1)^{k} \mathrm{Z}(\mathrm{M}(w, \varrho, \lambda) \varphi: x, w \beta, w \lambda)$ as required.

Suppose now that $\varrho=\operatorname{bc}\left(\sigma^{\prime}\right) \otimes \delta \otimes \overleftarrow{\delta}^{\tau}$ where $\sigma^{\prime} \in \Pi_{\mathrm{bc} \text {-gen }}^{\mathrm{G}_{m}^{\prime}}$ and $\delta=\delta_{1} \otimes \cdots \otimes \delta_{s} \in$ $\Pi^{\mathrm{M}_{\kappa}}$. Let $2 \kappa$ be the composition $\left(2 n_{1}, \ldots, 2 n_{s}\right)$ of $2 k$ and set ai $(\delta)=\operatorname{ai}\left(\delta_{1}\right) \otimes \cdots \otimes \operatorname{ai}\left(\delta_{s}\right) \in$ $\Pi^{\mathrm{M}_{2 \kappa}^{\prime}}$. We write $\mu \rightarrow \mu^{\prime}$ for the isomorphism $\mathfrak{a}_{\mathrm{M}_{\kappa}}^{*} \rightarrow \mathfrak{a}_{\mathrm{M}_{2 \kappa}^{\prime}}^{*}$ obtained by their identification with $\mathbf{R}^{s}$.

Corollary 5.6. - Let $\lambda=(z, \mu, \overleftarrow{\mu}) \in\left(\mathfrak{a}_{\tilde{\mathrm{L}}}^{*}\right)_{\mathbf{\mathbf { C }}}^{\widetilde{w_{*}}}$ with $z \in \mathbf{C}$ and $\mu \in \mathfrak{a}_{\mathbf{M}_{\kappa}, \mathbf{G}}^{*}$ and assume that $\mathrm{I}(\varrho, \lambda)$ is irreducible. Let $\beta \in \mathcal{E}_{\tilde{\mathrm{L}}}\left(\mathrm{X}_{\tilde{\mathrm{L}}}^{\tilde{\mathrm{M}}}, \varrho^{*}\right)$ and $\varrho^{\prime}=\sigma^{\prime}[z] \otimes \mathrm{I}_{\mathrm{M}_{2 \kappa}^{\prime}}\left(\operatorname{ai}(\delta), \mu^{\prime}\right)$. Then

$$
\mathcal{J}_{\varrho^{\prime}}\left(\mathrm{Z}^{\tilde{\mathrm{M}}}(\beta, \lambda), 0\right) \circ \Gamma_{\tilde{\mathrm{L}}, \tilde{\mathrm{M}}}(\varrho, \lambda) \circ \mathrm{N}(w, \varrho, \lambda)^{-1}=\eta(-1)^{k} \lambda_{\psi^{\prime-1}}^{-m k} \mathrm{Z}(w \beta, w \lambda) .
$$

Indeed, this follows from $(\mathbf{5 . 1 1})$ by observing, using $(\mathbf{4} .5),(\mathbf{3 . 9}),(\mathbf{3 . 1 0})$, and the fact that $\boldsymbol{\lambda}_{\psi^{\prime-1}}=\eta(-1) \boldsymbol{\lambda}_{\psi^{\prime}}$, that

$$
\mathfrak{n}\left(\varrho^{\prime}, 0\right)=\lambda_{\psi^{\prime-1}}^{-m k} \mathrm{C}_{\mathrm{M}}(w: \varrho, \lambda ; \psi) .
$$

For the rest of this section, we assume that $\mathrm{L}$ is even symmetric, i.e., $m=0$ in the notation above.

Let $\varrho \in \mathcal{R}(\mathrm{L})$ and $\beta \in \mathcal{E}_{\mathrm{L}}\left(\mathrm{X}_{\mathrm{L}}, \varrho^{*}\right)$. Let $\mathrm{P}=\mathrm{P}_{(k, k)}, \mathrm{M}=\mathrm{M}_{(k, k)}$ and $\mathrm{Q}_{\mathrm{M}}=\mathrm{Q} \cap \mathrm{M}$. Recall that $\mathrm{X}_{\mathrm{L}}=w_{0} \bullet \mathrm{L}$ and similarly for $\mathrm{M}$. For $\lambda \in\left(\mathfrak{a}_{\mathrm{L}}^{*}\right)_{\mathbf{C}}^{w_{0}}$ and $\varphi \in \mathrm{I}_{\mathrm{L}}^{\mathrm{M}}(\varrho)$ let

$$
\mathcal{F}(\varphi: x, \beta, \lambda)= \begin{cases}\int_{\mathrm{Q}_{\mathrm{M}}^{w_{0}} \backslash \mathrm{M}^{w_{0}}} \beta_{w_{0}}\left(\varphi_{\lambda}\left(h \iota_{w_{0}}^{x}\right)\right) d h & x=w_{0} \bullet \iota_{w_{0}}^{x}, \\ 0 & x \bullet \mathrm{G} \cap \mathrm{X}_{\mathrm{M}}=\emptyset .\end{cases}
$$

Since $\lambda \in\left(\mathfrak{a}_{\mathrm{L}}^{*}\right)_{\mathbf{C}}^{w_{0}}$, it easily follows that $\left\langle\lambda, \mathrm{H}\left(\mathrm{M}^{w_{0}}\right)\right\rangle=0$ and therefore,

$$
\mathcal{F}\left(\varphi: w_{0}, \beta, \lambda\right)=\int_{\mathrm{Q}_{\mathrm{M}}^{w_{0}} \backslash \mathrm{M}^{w_{0}}} \beta_{w_{0}}(\varphi(h)) d h
$$

is independent of $\lambda$.

Let $\imath: \mathrm{G}_{k} \rightarrow \mathrm{M}$ be the embedding given by $g^{l}=\operatorname{diag}\left(g, g^{\theta}\right)$. Then $\mathrm{M}^{w_{0}}=\mathrm{G}_{k}^{l}$, $\mathrm{Q}_{\mathrm{M}}^{w_{0}}=\mathrm{P}_{\kappa}^{\iota}$ and $\mathrm{L}^{w_{0}}=\mathrm{M}_{\kappa}^{\iota}$. In particular, $\mathrm{Q}_{\mathrm{M}}^{w_{0}}$ is a parabolic subgroup of $\mathrm{M}^{w_{0}}$ with $\delta_{\mathrm{Q}_{\mathrm{M}}}=$ $\left.\delta_{\mathrm{Q}_{\mathrm{M}}}^{\frac{1}{2}}\right|_{\mathrm{Q}_{\mathrm{M}}} ^{w_{0}}$. Furthermore, $\langle\lambda, \mathrm{H}(h)\rangle=0$ for all $h \in \mathrm{Q}_{\mathrm{M}}^{w_{0}}$. It follows that the integral in $(\mathbf{5 . 1 5})$ is well defined and $\mathcal{F}(\beta, \lambda) \in \mathcal{E}_{\mathrm{M}}\left(\mathrm{X}_{\mathrm{M}}, \mathrm{I}_{\mathrm{L}}^{\mathrm{M}}(\varrho, \lambda)^{*}\right)$ is holomorphic in $\lambda$.

Lemma 5.7. - Let $\varrho \in \mathcal{R}(\mathrm{L}), \beta \in \mathcal{E}_{\mathrm{L}}\left(\mathrm{X}_{\mathrm{L}}, \varrho^{*}\right)$ and $\lambda \in\left(\mathfrak{a}_{\mathrm{L}}^{*}\right)_{\mathbf{C}}^{w_{0}}$. We have

$$
\mathrm{Z}_{\mathrm{L}}^{\mathrm{G}}(\beta, \lambda)=\mathrm{Z}_{\mathrm{M}}^{\mathrm{G}}(\mathcal{F}(\beta, \lambda), 0) \circ \Gamma_{\mathrm{L}, \mathrm{M}}(\varrho, \lambda) .
$$


Proof. — Both sides are elements of $\mathcal{E}_{\mathrm{G}}\left(\mathrm{X}, \mathrm{I}(\varrho, \lambda)^{*}\right)$ which are supported on $w_{0} \bullet \mathrm{G}$. Therefore, it suffices to show that

$$
\mathrm{Z}_{\mathrm{L}}^{\mathrm{G}}\left(w_{0}, \beta, \lambda\right)=\mathrm{Z}_{\mathrm{M}}\left(w_{0}, \mathcal{F}\left(\beta, \lambda^{\mathrm{M}}\right), \lambda_{\mathrm{M}}\right) \circ \Gamma_{\mathrm{L}, \mathrm{M}}(\varrho, \lambda)
$$

Note that $\mathrm{Q}^{w_{0}}=\mathrm{Q}_{\mathrm{M}}^{w_{0}} \mathrm{U}^{w_{0}}, \mathrm{P}^{w_{0}}=\mathrm{M}^{w_{0}} \mathrm{U}^{w_{0}}$ and $\delta_{\mathrm{P}} w_{0}=\left.\delta_{\mathrm{P}}^{\frac{1}{2}}\right|_{\mathrm{P}} ^{w_{0}}$. Thus, for any $\varphi \in \mathrm{I}_{\mathrm{L}}^{\mathrm{G}}(\varrho)$ we have

$$
\begin{aligned}
\mathcal{F}\left(\varphi_{\mathrm{M}}: w_{0}, \beta, \lambda\right) & =\int_{\mathrm{Q}_{\mathrm{M}}^{w_{0}} \backslash \mathrm{M}^{w_{0}}} \delta_{\mathrm{P}}^{-\frac{1}{2}}(h) \beta_{w_{0}}\left(\varphi_{\lambda}(h)\right) d h \\
& =\int_{\mathrm{Q}^{w_{0}} \backslash \mathrm{P}^{w_{0}}} \delta_{\mathrm{P}^{w_{0}}}^{-1}(p) \beta_{w_{0}}\left(\varphi_{\lambda}(p)\right) d p
\end{aligned}
$$

where we recall that $\varphi_{\mathrm{M}}=\left.\delta_{\mathrm{P}}^{-\frac{1}{2}} \varphi\right|_{\mathrm{M}} \in \mathrm{I}_{\mathrm{L}}^{\mathrm{M}}(\varrho)$. Therefore, by $(\mathbf{1 . 2})$ and $(\mathbf{5 . 6})$ we have

$$
\begin{aligned}
\mathrm{Z}_{\mathrm{M}} & \left(\Gamma_{\mathrm{L}, \mathrm{M}}(\varrho, \lambda) \varphi: w_{0}, \mathcal{F}(\beta, \lambda), 0\right) \\
\quad= & \int_{\mathrm{P}^{w_{0}} \backslash \mathrm{G}^{w_{0}}} \mathcal{F}\left([\mathrm{I}(h, \varrho, \lambda) \varphi]_{\mathrm{M}}: w_{0}, \beta, \lambda\right) d h \\
\quad= & \int_{\mathrm{P}^{w_{0}} \backslash \mathrm{G}^{w_{0}}} \int_{\mathrm{Q}^{w_{0} \backslash \mathrm{P}^{w_{0}}}} \delta_{\mathrm{P}^{w_{0}}}^{-1}(p) \beta_{w_{0}}\left(\varphi_{\lambda}(p h)\right) d p d h \\
& =\int_{\mathrm{Q}^{w_{0}} \backslash \mathrm{G}} \beta_{w_{0}}\left(\varphi_{\lambda}(h)\right) d h
\end{aligned}
$$

which, once again by $(\mathbf{5 . 6})$, equals $\mathrm{Z}_{\mathrm{L}}^{\mathrm{G}}\left(\varphi: w_{0}, \beta, \lambda\right)$ as required.

5.3. The normalized closed periods. - We continue to assume that $\mathrm{L}=\mathrm{M}_{(\kappa, \overleftarrow{\kappa})}$ is even symmetric and let $\mathbf{M}=\mathbf{M}_{(k, k)}$ as before. Recall that $\mathrm{L}^{w_{0}}=\left\{\operatorname{diag}(m, \theta(m)): m \in \mathbf{M}_{\kappa}\right\}$. Denote by $\Pi^{\mathrm{L}, w_{0}}$ the set of all $\varrho \in \Pi^{\mathrm{L}}$ that are $\mathrm{L}^{w_{0}}$-distinguished. Concretely,

$$
\Pi^{\mathrm{L}, w_{0}}=\left\{\sigma_{1} \otimes \cdots \otimes \sigma_{s} \otimes \sigma_{s}^{\tau} \otimes \cdots \otimes \sigma_{1}^{\tau}: \sigma_{i} \in \Pi^{\mathrm{G}_{n_{i}}}\right\} .
$$

Let $\Pi_{\text {gen }}^{\mathrm{L}, w_{0}}=\Pi^{\mathrm{L}, w_{0}} \cap \Pi_{\text {gen }}^{\mathrm{L}}$. Let $b$ be the involution $\operatorname{diag}\left(g_{1}, g_{2}\right)^{\mathrm{b}}=\operatorname{diag}\left(g_{1}, g_{2}^{\tau}\right)$ of $\mathbf{M}\left(g_{1}, g_{2} \in \mathbf{G}_{k}\right)$.

For $\varrho \in \Pi_{\text {gen }}^{\mathrm{L}, w_{0}}$, we define the normalized closed period $\beta^{\varrho}$ by

$$
\beta_{w_{0} \bullet}^{\varrho}(\mathrm{W})=\beta_{0}^{\varrho^{\mathrm{b}}}\left((\mathcal{W}(\varrho, l) \mathrm{W})^{b}\right)
$$

where $\beta_{0}^{\varrho^{b}}$ is defined in $(\mathbf{A . 1 0})$ (except that now we use it with respect to E) and $\mathrm{W}^{\mathrm{b}} \in$ $\mathcal{W}\left(\varrho^{b}\right)$ is given by $\mathrm{W}^{b}\left(l^{b}\right)=\mathrm{W}(l), l \in \mathrm{L}$. Thus, $0 \not \equiv \beta^{\varrho} \in \mathcal{E}_{\mathrm{L}}\left(\mathrm{X}_{\mathrm{L}}, \mathcal{W}(\varrho)^{*}\right)$. 
and

Lemma 5.8. - Let $\varrho \in \Pi_{\text {gen }}^{\mathrm{L}, w_{0}}, \lambda \in\left(\mathfrak{a}_{\mathrm{L}}^{*}\right)_{\mathbf{G}}^{w_{0}}$ and $\sigma=\mathrm{I}_{\mathrm{L}}^{\mathrm{M}}(\varrho, \lambda) \in \Pi^{\mathrm{M}}$. Then $\sigma \in \Pi_{\text {gen }}^{\mathrm{M}, w_{0}}$

$$
\beta^{\sigma} \circ \mathbf{W}_{\mathrm{L}}^{\mathrm{M}}(\varrho, \lambda)=\mathcal{F}\left(\beta^{\varrho}, \lambda\right)
$$

Proof. - It suffices to prove this equality at $w_{0}$. This case amounts to Corollary A.5 upon applying $b$.

For $\varrho \in \Pi_{\text {gen }}^{\mathrm{L}, w_{0}}$, we can now define the relative closed Bessel distribution

$$
\tilde{\mathbf{D}}_{\mathrm{L}}(\varrho, \lambda)=\tilde{\mathbf{D}}(\varrho, \lambda)=\lambda_{\psi^{\prime}}^{k} \tilde{\mathfrak{B}}_{\mathrm{I}(\mathfrak{W}(\varrho), \lambda)}^{Z\left(\beta^{e}, \lambda\right), \mathbf{W}_{e}^{\psi^{-1}}\left(\varrho^{\vee},-\lambda\right)}, \quad \lambda \in\left(\mathfrak{a}_{\mathrm{L}}^{*}\right)_{\mathbf{G}}^{w_{0}}
$$

It is holomorphic in $\lambda$. The factor $\lambda_{\psi^{\prime}}^{k}$ is needed for comparison with $\mathrm{G}^{\prime}-\mathrm{cf}$. Section 9.

We may reduce the study of the closed Bessel distribution to the case $\mathrm{L}=\mathrm{M}$ as follows.

Proposition 5.9. - We have $\tilde{\mathbf{D}}_{\mathrm{L}}(\varrho, \lambda)=\tilde{\mathbf{D}}_{\mathrm{M}}\left(\mathrm{I}_{\mathrm{L}}^{\mathrm{M}}(\varrho, \lambda), 0\right)$.

Proof. - Let $\sigma=\mathrm{I}_{\mathrm{L}}^{\mathrm{M}}(\varrho, \lambda)$. We will apply the relation $(\mathbf{2 . 9})$ to the equivalence

$$
\left(\mathcal{A}_{\varrho}(\lambda), \mathcal{A}_{\varrho^{\vee}}(-\lambda)\right)
$$

of dual couples where

$$
\mathcal{A}_{\varrho}(\lambda)=\mathrm{I}_{\mathrm{M}}^{\mathrm{G}}\left(\mathbf{W}_{\mathrm{L}}^{\mathrm{M}}(\varrho, \lambda)\right) \circ \Gamma_{\mathrm{L}, \mathrm{M}}^{\mathrm{G}}(\mathcal{W}(\varrho), \lambda): \mathrm{I}_{\mathrm{L}}^{\mathrm{G}}(\mathcal{W}(\varrho), \lambda) \rightarrow \mathrm{I}_{\mathrm{M}}^{\mathrm{G}}(\mathcal{W}(\sigma), 0) .
$$

Recall that by $(\mathbf{1 . 6})$ we have

$$
\mathbf{W}_{e}(\varrho, \lambda)=\mathbf{W}_{e}(\sigma, 0) \circ \mathcal{A}_{\varrho}(\lambda) .
$$

On the other hand, by Lemmas 5.7 and 5.8 we have

$$
\mathrm{Z}_{\mathrm{L}}\left(\beta^{\varrho}, \lambda\right)=\mathrm{Z}_{\mathrm{M}}\left(\beta^{\sigma}, 0\right) \circ \mathcal{A}_{\varrho}(\lambda)
$$

The Proposition follows.

Corollary 5.10. - Let $\mathrm{L}_{1} \subseteq \mathrm{L}$ be both even symmetric, $\sigma \in \Pi_{\text {gen }}^{\mathrm{L}_{1}, w_{0}}$ and $\mu \in\left(\mathfrak{a}_{\mathrm{L}_{1}}^{*}\right)_{\mathbf{G}}^{w_{0}}$ be such that $\varrho=\mathrm{I}_{\mathrm{L}_{1}}^{\mathrm{L}}(\sigma, \mu) \in \Pi^{\mathrm{L}}$. Then

$$
\tilde{\mathbf{D}}_{\mathrm{L}}(\varrho, \lambda)=\tilde{\mathbf{D}}_{\mathrm{L}_{1}}(\sigma, \mu+\lambda), \quad \lambda \in\left(\mathfrak{a}_{\mathrm{L}}^{*}\right)_{\mathbf{C}}^{w_{0}} .
$$

Indeed, both sides are equal to $\tilde{\mathbf{D}}_{\mathrm{M}}\left(\mathrm{I}_{\mathrm{L}}^{\mathrm{M}}(\varrho, \lambda), 0\right)$. 


\section{Applications of the geometric lemma}

In this section, we reduce the study of local unitary periods in the $p$-adic case to the case of pure Galois-invariant representations (i.e., with supercuspidal support contained in $\sigma[\mathbf{Z}]$ for a Galois-invariant supercuspidal representation $\sigma$ ). Once the supercuspidal case is settled, the main tool will be the geometric Lemma of Bernstein-Zelevinsky [BZ77]. On the other hand, the supercuspidal case requires a global argument (which however does not require the relative trace formula comparison).

Assume from now on that $\mathrm{F}$ is $p$-adic and $\mathrm{E} / \mathrm{F}$ is inert. Our main goal in this section is the following result.

Theorem 6.1. - Let $\pi \in \Pi$ and $x \in \mathrm{X}$.

(1) If $\pi$ is $\mathrm{G}^{x}$-distinguished then $\pi \simeq \pi^{\tau}$.

(2) If $\pi$ is totally $\tau$-isotropic (see Section 3.2) then $\mathcal{E}_{\mathrm{G}}\left(\mathrm{X}, \pi^{*}\right)$ is one-dimensional and

$$
\operatorname{dim} \operatorname{Hom}_{\mathrm{G}^{x}}(\pi, \mathbf{C})= \begin{cases}1 & \text { if } \mathrm{G}^{x} \text { is quasi-split }, \\ 0 & \text { otherwise. }\end{cases}
$$

Consider first the supercuspidal case. We turn to a global setting. Assume that $\mathrm{E} / \mathrm{F}$ is a quadratic extension of number fields and $v_{0}$ is an inert place of $\mathrm{F}$ such that $\mathrm{E}_{v_{0}} / \mathrm{F}_{v_{0}}$ is isomorphic to our given quadratic extension of $p$-adic fields. Consider $\pi \in \Pi_{\text {cusp }}^{\mathrm{G}_{v_{0}}}$ and $x \in \mathrm{X}_{v_{0}}$ such that $\pi$ is $\mathrm{G}_{v_{0}}^{x}$-distinguished. After possibly twisting by an unramified character, we may assume that $\pi$ is unitary. By passing to a different representative in the $\mathrm{G}_{v_{0}}$-orbit, we may assume that $x \in \mathrm{X}$. It follows from [HM02a, Theorem 1] that there exists an irreducible cuspidal automorphic representation $\sigma=\bigotimes_{v} \sigma_{v}$ of $\mathrm{G}_{\mathbf{A}}$ which is distinguished by $\mathrm{G}^{x}$ and such that $\sigma_{v_{0}} \simeq \pi$. A well-known argument from [HLR86] using strong multiplicity one (e.g., [Jac05a, Theorem 4]) yields that $\sigma$ (and in particular $\pi)$ is Galois invariant.

For the rest of the proof we return to the local setting. Recall the involution $\theta=\theta_{k}$ of $\mathrm{G}_{k}$.

Let $\sigma \in \mathcal{R}(\mathrm{M})$ and $\pi=\mathrm{I}(\sigma, 0)$. By [BZ77, Theorem 5.2], for $x \in \mathrm{X}, \pi$ admits a filtration by $\mathrm{G}^{x}$-invariant subspaces which is parameterized by $\mathrm{P} \backslash \mathrm{G} / \mathrm{G}^{x}$. The successive quotients can be described in terms of induction and Jacquet functors. We study $\mathrm{G}^{x}$-invariant functionals on $\pi$ through these subquotients.

6.1. $\mathrm{P}$-orbits in $\mathrm{X}$. - The $\mathrm{P}$-orbits in $\mathrm{X}$ were analyzed in [LR03, Proposition 4.2.1] building on earlier results of Springer [Spr85]. (For a more general setup - $\mathrm{cf}$. [HW93].) To describe the results, fix $\mathrm{M}=\mathrm{M}_{\kappa}$ where $\kappa=\left(n_{1}, \ldots, n_{t}\right)$, and let $\mathrm{W}_{2}[\mathrm{M}]$ denote the set of involutions $w$ in $\mathrm{W}$ satisfying $w \alpha<0$ for all $\alpha \in \Delta_{0}^{\mathrm{M}}$, i.e., $w$ is the longest element in the double coset $\mathrm{W}^{\mathrm{M}} w \mathrm{~W}^{\mathrm{M}}$. For $w \in \mathrm{W}_{2}[\mathrm{M}]$ the group 


$$
\mathrm{M}(w)=\mathrm{M} \cap w \mathrm{M} w
$$

is a standard Levi subgroup of M invariant under conjugation by $w$.

Lemma 6.2. - [LR03, Proposition 4.2.1 $]^{7}$ Every $\mathrm{P}$-orbit $x \bullet \mathrm{P}$ in $\mathrm{X}$ intersects $w \mathrm{M}(w)$ for a unique $w \in \mathrm{W}_{2}[\mathrm{M}]$. We say that $x$ (or its $\mathrm{P}$-orbit) lies above $w$ in this case. If $x$ lies above $w$ then $w \mathrm{M}(w) \cap x \bullet \mathrm{P}$ is a single $\mathrm{M}(w)$-orbit.

Thus, the $\mathrm{P}$-orbits in $\mathrm{X}$ are parameterized by the disjoint union over all $w \in$ $\mathrm{W}_{2}[\mathrm{M}]$ of the $\mathrm{M}(w)$-orbits in $w \mathrm{M}(w) \cap \mathrm{X}$. Fix $w \in \mathrm{W}_{2}[\mathrm{M}]$ and set $\mathrm{L}=\mathrm{M}(w)$. Recall that $\mathrm{L}=\mathrm{M}_{\gamma}$ for a composition $\gamma=\left(\gamma_{1}, \ldots, \gamma_{t}\right)$ of $n$ refining $\kappa$, i.e., for all $i=1, \ldots, t$, $\gamma_{i}=\left(k_{i, 1}, \ldots, k_{i, s_{i}}\right)$ is a composition of $n_{i}$. Thus, the blocks of $\mathrm{L}$ are enumerated by the linearly ordered set $(\mathfrak{I}=\mathfrak{I}(w), \prec)$ where

$$
\mathfrak{I}=\left\{(i, j): i=1, \ldots, t, j=1, \ldots, s_{i}\right\}
$$

and $\prec$ is the lexicographic order. We write an element of $\mathrm{L}$ as $\operatorname{diag}\left(g_{\imath}\right)_{l \in \mathfrak{I}}$ where $g_{\imath} \in \mathrm{G}_{k_{l}}$. Note that $w$ defines an involution on $\mathfrak{I}$ (also denoted by $w$ ) such that $k_{w(l)}=k_{l}$,

$$
w \operatorname{diag}\left(g_{l}\right)_{l \in \mathfrak{I}} w=\operatorname{diag}\left(w_{0}^{\mathrm{G}_{k_{l}}} g_{w(l)} w_{0}^{\mathrm{G}_{k_{l}}}\right)_{l \in \mathfrak{I}}
$$

and

$$
w(i, j+1) \prec w(i, j), \quad i=1, \ldots, t, j=1, \ldots, s_{i}-1 .
$$

In particular, for any $i$ there is at most one $j$ such that $w(i, j)=(i, j)$. For $m=$ $\operatorname{diag}\left(m_{l}\right)_{l \in \mathfrak{I}} \in \mathrm{L}$, the element $w m \in w \mathrm{~L}$ lies in $\mathrm{X}$ if and only if

$$
m_{w(l)}=\theta\left(m_{l}^{-1}\right), \quad l \in \mathfrak{I} .
$$

Furthermore, if $w m \in \mathrm{X}$ and $g=\operatorname{diag}\left(g_{l}\right)_{l \in \mathfrak{I}} \in \mathrm{L}$ then

$$
(w m) \bullet g=w \operatorname{diag}\left(\left(\theta\left(g_{w(l)}^{-1}\right) m_{\iota} g_{l}\right)_{\imath \in \mathfrak{I}}\right) .
$$

Let $\mathfrak{F}_{w}$ be the set of fixed points of $w$ on $\mathfrak{I}$. We can choose $g$ so that $\theta\left(g_{w(l)}^{-1}\right) m_{l} g_{l}=e$ for all $\imath \notin \mathfrak{F}_{w}$. Thus, the L-orbits in $w \mathrm{~L} \cap \mathrm{X}$ are parameterized by the product over $\imath \in \mathfrak{F}_{w}$ of the $\mathrm{G}_{k_{\imath}}$-orbits on $\mathrm{X}_{k_{\imath}}$ or, what amounts to the same, the $\mathrm{G}_{k_{\imath}}$-orbits on $w_{\imath} \mathrm{X}_{k_{\iota}}$ (where $w_{\imath}=w_{0}^{\mathrm{G}_{k_{l}}}$ ) with respect to the twisted action $\left(x^{\prime}, g\right) \mapsto \theta\left(g^{-1}\right) x^{\prime} g$. Since we are in the $p$-adic case, they are therefore parameterized by $\left(\mathrm{F}^{*} / \mathrm{NmE}^{*}\right)^{\mathfrak{F}_{w}}$. The stabilizer $\mathrm{L}^{y}$ of a representative of the form

$$
y=w \operatorname{diag}\left(\left(m_{\iota}\right)_{\iota \in \mathfrak{I}}\right), \quad m_{\iota}=e \text { for all } \iota \notin \mathfrak{F}_{w}
$$

${ }^{7}$ In [ibid.], the left action $g \star y=g y \theta\left(g^{-1}\right)$ of $\mathrm{G}$ on $\mathrm{Y}=\left\{g \in \mathrm{G}: g=\theta\left(g^{-1}\right)\right\}=\mathrm{X} w_{0}$ was considered instead of our right action of $\mathrm{G}$ on $\mathrm{X}$. Thus, to translate the results of [ibid.] to our setting, we identify $\mathrm{Y}$ and $\mathrm{X}$ via the map $y \mapsto x=\left(y w_{0}\right)^{-1}$. 
is the subgroup of elements $g=\operatorname{diag}\left(g_{l}\right)$ such that for all $\iota \in \mathfrak{I}$ we have

$$
\begin{cases}g_{\imath} \in \mathrm{G}_{k_{l}}^{w_{l} m_{l}} & \text { if } \iota \in \mathfrak{F}_{w}, \\ g_{w(l)}=\theta\left(g_{l}\right) & \text { otherwise. }\end{cases}
$$

Remark 6.3. - The above analysis works over any $\mathrm{F}$ of characteristic 0 . The only difference is the description of $\mathrm{X}_{k} / \mathrm{G}_{k}$ where the discriminant map $\mathrm{X}_{k} / \mathrm{G}_{k} \rightarrow \mathrm{F}^{*} / \mathrm{Nm} \mathrm{E}^{*}$ may not be injective. In particular, in the case $\mathrm{P}=\mathrm{P}_{0}$ there is no difference between the $p$-adic case and the Archimedean case.

The open $\mathrm{P}$-orbits in $\mathrm{X}$ are precisely those which lie above $w=w_{0}^{\mathrm{M}}$. There are exactly $2^{t}$ open orbits and their representatives can be taken in $\mathrm{X}^{\mathrm{M}} / \mathrm{M}$. Recall that $\mathrm{X}^{\circ}=$ $\mathrm{X}^{\mathrm{M}} \bullet \mathrm{P}$ (the F-points of the Zariski open orbit $\mathbf{X}^{\circ}$ of $\mathbf{P}$ on $\mathbf{X}$ ) is the union of the open P-orbits in $\mathrm{X}$ and for $x \in \mathrm{X}$ we set

$$
\mathrm{G}^{\circ}[x]=\left\{g \in \mathrm{G}: x \bullet g^{-1} \in \mathrm{X}^{\circ}\right\}
$$

which is the union of the $2^{t-1}$ open double cosets in $P \backslash G / G^{x}$.

A P-orbit that lies above $w=w_{0}$ is closed. There is a single orbit that lies above $w_{0}$ if $n$ is even and $\mathrm{M} \subseteq \mathrm{G}_{n / 2} \times \mathrm{G}_{n / 2}$ and there are precisely two such orbits otherwise (one in each G-orbit).

6.2. The geometric Lemma. - Suppose that $(\pi, \mathcal{V})$ is an induced representation $\mathrm{I}_{\mathrm{M}}^{\mathrm{G}}(\sigma)$. For $x \in \mathrm{X}$ we describe a filtration for the restriction of $\pi$ to $\mathrm{G}^{x}$.

We identify the double coset space $\mathrm{P} \backslash \mathrm{G} / \mathrm{G}^{x}$ with the set $(x \bullet \mathrm{G}) / \mathrm{P}$ of P-orbits on $x \bullet \mathrm{G}$ via $g \mapsto x \bullet g^{-1}$. By [BZ76, $\left.\S 1.5\right]$, there is an ordering $\left\{\epsilon_{i}\right\}_{i=1}^{k}$ of double coset representatives of $\mathrm{P} \backslash \mathrm{G} / \mathrm{G}^{x}$ such that

$$
\mathrm{Y}_{i}=\bigcup_{j=1}^{i} \mathrm{P} \epsilon_{j} \mathrm{G}^{x}
$$

is open in $\mathrm{G}$ for all $i=1, \ldots, k$. We take the first $2^{t-1} \epsilon_{i}$ 's to lie in $\mathrm{G}^{\circ}[x]$. If $x \in w_{0} \bullet \mathrm{G}$, we also take $\epsilon_{k}$ such that $x=w_{0} \bullet \epsilon_{k}$. Let

$$
\mathcal{V}_{i}=\left\{\varphi \in \mathcal{V}: \operatorname{supp}(\varphi) \subseteq \mathrm{Y}_{i}\right\}
$$

In particular,

$$
\mathcal{V}_{2^{t-1}}=\left\{\varphi \in \mathcal{V}: \operatorname{supp}(\varphi) \subseteq \mathrm{G}^{\circ}[x]\right\}
$$

and

$$
\mathcal{V}_{k-1}=\left\{\varphi \in \mathcal{V}:\left.\varphi\right|_{\epsilon_{k} \mathrm{G}^{x}} \equiv 0\right\}
$$


The sequence

$$
0=\mathcal{V}_{0} \subseteq \mathcal{V}_{1} \subseteq \cdots \subseteq \mathcal{V}_{k}=\mathcal{V}
$$

is a filtration of $\mathcal{V}$ by $\mathrm{G}^{x}$-invariant subspaces and the successive quotients are described as follows (see [BZ77, Theorem 5.2]). Fix $i=1, \ldots, k$ and choose $y$ in the P-orbit of $x \bullet \epsilon_{i}^{-1}$ and a representative $\iota_{y}^{x} \in \mathrm{G}$ such that $x=y \bullet \iota_{y}^{x}$. Thus, $\iota_{y}^{x} \in \mathrm{P} \epsilon_{i} \mathrm{G}^{x}$. For groups $\mathrm{A} \subseteq \mathrm{B}$, a representation $\varrho$ of $\mathrm{A}$ and $b \in \mathrm{B}$ we let $\varrho^{b}$ denote the representation of $b^{-1} \mathrm{~A} b$ on the space of $\varrho$ given by $\varrho^{b}\left(b^{-1} a b\right)=\varrho(a), a \in \mathrm{A}$. Then

$$
\begin{aligned}
\mathcal{V}_{i} / \mathcal{V}_{i-1} & \simeq \mathrm{V}_{y}^{\sigma}[x]:=\operatorname{ind}_{\mathrm{G}^{x} \cap\left(y_{y}^{x}\right)^{-1} \mathrm{P}_{y}^{x}}^{\mathrm{G}^{x}}\left(\left[\left.\sigma \cdot \delta_{\mathrm{P}}^{1 / 2}\right|_{\mathrm{P}^{y}}\right]^{l_{y}^{x}}\right) \\
& =\operatorname{ind}_{\left(l_{y}^{x}\right)^{-1} \mathrm{P}_{l_{y}^{x}}^{\mathrm{G}^{x}}}\left(\left[\left.\sigma \cdot \delta_{\mathrm{P}}^{1 / 2}\right|_{\mathrm{P}^{y}}\right]^{l_{y}^{x}}\right) \simeq\left(\operatorname{ind}_{\mathrm{P}}^{\mathrm{G}^{y}}\left(\left[\left.\sigma \cdot \delta_{\mathrm{P}}^{1 / 2}\right|_{\mathrm{P}}\right]\right)\right)^{l_{y}^{x}}
\end{aligned}
$$

where ind denotes unnormalized induction with compact support. In particular, the $\mathrm{G}^{x}$ space $\mathrm{V}_{y}^{\sigma}[x]$ depends only on the orbit $y \bullet \mathrm{P}$ and not on the choice of $y$ or $\iota_{y}^{x}$.

Recall that by Lemma 6.2, any P-orbit $\mathcal{O} \in \mathrm{X} / \mathrm{P}$ lies above some $w \in \mathrm{W}_{2}[\mathrm{M}]$ and we may choose a representative $y \in \mathcal{O} \cap w \mathrm{M}(w)$. Recall that $r_{\mathrm{L}, \mathrm{M}}: \mathcal{R}(\mathrm{M}) \rightarrow \mathcal{R}(\mathrm{L})$ is the normalized Jacquet functor.

Lemma 6.4. - Let $\sigma \in \mathcal{R}(\mathrm{M})$ and let $w \in \mathrm{W}_{2}[\mathrm{M}]$. Set $\mathrm{L}=\mathrm{M}(w)$ and let $y \in w \mathrm{~L} \cap \mathrm{X}$. Then there is an isomorphism of vector spaces

$$
\operatorname{Hom}_{\mathrm{G}^{x}}\left(\mathrm{~V}_{y}^{\sigma}[x], \mathbf{G}\right) \simeq \operatorname{Hom}_{\mathrm{L}^{y}}\left(r_{\mathrm{L}, \mathrm{M}}(\sigma), \mathbf{G}\right) .
$$

Proof. - Let $\mathrm{Q}=\mathrm{P}_{\gamma}=\mathrm{LV}$ with $\mathrm{V}=\mathrm{U}_{\gamma}$. Thus, $\mathrm{Q} \subseteq \mathrm{P}$. Let $\mathrm{R}$ be the unipotent radical of $\mathrm{P}^{y}$ and let $\operatorname{proj}_{\mathrm{M}}: \mathrm{P}^{y} \rightarrow \mathrm{M}$ be the projection map to the Levi subgroup $\mathrm{M}$ of $\mathrm{P}$. Set also $V_{1}=M \cap V$, so that $V=V_{1} U$ and $L V_{1}$ is the standard Levi decomposition of $\mathrm{Q} \cap \mathrm{M}$, which is a standard parabolic subgroup of M. By [LR03, Proposition 4.2.2] (adapted to our setup) we have $\mathrm{P}^{y}=\mathrm{Q}^{y}=\mathrm{L}^{y} \ltimes \mathrm{R}$ and $\operatorname{proj}_{\mathrm{M}}(\mathrm{R})=\mathrm{V}_{1}$. Since $w \mathrm{~L} w=\mathrm{L}$, it follows from [ibid., Proposition 4.3.2] that $\delta_{\mathrm{P}^{y}}=\delta_{\mathrm{Q}^{y}}=\left.\delta_{\mathrm{Q}}^{1 / 2}\right|_{\mathrm{P}^{y}}$. Therefore, by Frobenius reciprocity and $(\mathbf{6 . 3})$ we have

$$
\begin{aligned}
& \operatorname{Hom}_{\mathrm{G}^{x}}\left(\mathrm{~V}_{y}^{\sigma}[x], \mathbf{G}\right) \simeq \operatorname{Hom}_{\mathrm{G}^{y}}\left(\mathrm{~V}_{y}^{\sigma}[y], \mathbf{C}\right) \simeq \operatorname{Hom}_{\mathrm{P}^{y}}\left(\left.\sigma \cdot\left(\delta_{\mathrm{P}}^{\frac{1}{2}} \delta_{\mathrm{Q}^{-\frac{1}{2}}}\right)\right|_{\mathrm{P}^{y}}, \mathbf{G}\right) \\
&=\operatorname{Hom}_{\mathrm{L}^{y} \mathrm{~V}_{1}}\left(\left.\sigma \cdot\left(\delta_{\mathrm{P}}^{\frac{1}{2}} \delta_{\mathrm{Q}}^{-\frac{1}{2}}\right)\right|_{\mathrm{L}^{y} \mathrm{~V}_{1}}, \mathbf{C}\right)=\operatorname{Hom}_{\mathrm{L}}\left(r_{\mathrm{L}, \mathrm{M}}(\sigma), \mathbf{C}\right)
\end{aligned}
$$

where for the third isomorphism we used that $\operatorname{proj}_{M}(R)=V_{1}$ and the last equality follows from the definition of the normalized Jacquet functor. Altogether, we obtain the Lemma.

Definition 6.5. - We say that $y \in \mathrm{X}$ (or $y \bullet \mathrm{P} \in \mathrm{X} / \mathrm{P})$ is relevant for $\sigma$ (or for $\pi$ if $\mathrm{P}$ is clear from the context) if $\operatorname{Hom}_{\mathrm{G}^{y}}\left(\mathrm{~V}_{y}^{\sigma}[y], \mathbf{C}\right) \neq 0$ or equivalently, if $\operatorname{Hom}_{\mathrm{G}^{x}}\left(\mathrm{~V}_{y}^{\sigma}[x], \mathbf{G}\right) \neq 0$ for some $x \in y \bullet \mathrm{G}$. 
By Lemma 6.4, in its notation, we have $y$ is relevant for $\sigma$ if and only if $r_{\mathrm{L}, \mathrm{M}}(\sigma)$ is $\mathrm{L}^{y}$-distinguished.

For $w \in \mathrm{W}_{2}[\mathrm{M}]$ set $\mathrm{L}=\mathrm{M}(w)$ and let $y \in w \mathrm{~L} \cap \mathrm{X}$ be a representative as in (6.2). With the notation of Section 6.1, an irreducible representation $\varrho=\bigotimes_{l \in \Im(w)} \varrho_{l}$ of $\mathrm{L}$ is $\mathrm{L}^{y}$-distinguished if and only if for every $\iota \in \mathfrak{I}$ we have

$$
\begin{cases}\varrho_{\imath} \text { is }\left(\mathrm{G}_{k_{l}}\right)^{w_{l} m_{l}} \text {-distinguished } & \text { if } w(\imath)=\imath, \\ \varrho_{w(l)} \simeq \varrho_{l}^{\tau} & \text { otherwise }\end{cases}
$$

Definition 6.6. - We say that the unitary periods on $\pi=\mathrm{I}(\sigma, 0)$ are supported on open $\mathrm{P}$-orbits (or simply supported on open orbits if $\mathrm{P}$ is clear from the context) if the non-open orbits are not relevant. Similarly, we say that the unitary periods on $\pi=\mathrm{I}(\sigma, 0)$ are supported on $w_{0} \bullet \mathrm{P}$ if the only possibly relevant orbit is $w_{0} \bullet \mathrm{P}$.

We can of course relate the open and closed local periods defined in the previous sections to the geometric Lemma. Identify $\mathcal{E}_{\mathrm{M}}\left(\mathrm{M} \cap x \bullet \mathrm{G}, \sigma^{*}\right)$ with the subspace of $\alpha \in$ $\mathcal{E}_{\mathrm{M}}\left(\mathrm{X}^{\mathrm{M}}, \sigma^{*}\right)$ such that $\alpha_{y} \equiv 0$ for all $y \in \mathrm{X}^{\mathrm{M}} \backslash x \bullet \mathrm{G}$. Let $\mathrm{J}^{\circ}(x, \alpha, \lambda)$ be the restriction of $\mathrm{J}(x, \alpha, \lambda)$ to $\mathcal{V}_{2^{t-1}}$. Note that for $\varphi \in \mathcal{V}_{2^{t-1}}$ the integrands in (4.2) are compactly supported and therefore the integral is convergent and $\mathrm{J}^{\circ}(x, \alpha, \lambda)$ is entire in $\lambda$. It follows that

$$
\text { the map } \alpha \mapsto \mathrm{J}^{\circ}(x, \alpha, 0) \text { defines an isomorphism }
$$

$$
\mathcal{E}_{\mathrm{M}}\left(\mathrm{M} \cap x \bullet \mathrm{G}, \sigma^{*}\right) \simeq \operatorname{Hom}_{\mathrm{G}^{x}}\left(\mathcal{V}_{2^{t-1}}, \mathbf{G}\right) .
$$

Lemma 6.7. - Suppose that the unitary periods on $\pi$ are supported on open $\mathrm{P}$-orbits. Then

(1) The restriction map

$$
\operatorname{Hom}_{\mathrm{G}^{x}}(\pi, \mathbf{G}) \rightarrow \operatorname{Hom}_{\mathrm{G}^{x}}\left(\mathcal{V}_{2^{t-1}}, \mathbf{G}\right)
$$

is an isomorphism for any $x \in \mathrm{X}$.

(2) For every $\alpha \in \mathcal{E}_{\mathrm{M}}\left(\mathrm{X}^{\mathrm{M}}, \sigma^{*}\right), \mathrm{J}(\alpha, \lambda)$ is holomorphic at $\lambda=0$ and $\alpha \mapsto \mathrm{J}(\alpha, 0)$ is a vector space isomorphism between $\mathcal{E}_{\mathrm{M}}\left(\mathrm{X}^{\mathrm{M}}, \sigma^{*}\right)$ and $\mathcal{E}_{\mathrm{G}}\left(\mathrm{X}, \pi^{*}\right)$.

(3) For every $x \in \mathrm{X}$, the map $\alpha \mapsto \mathrm{J}(x, \alpha, 0)$ is a vector space isomorphism between $\mathcal{E}_{\mathrm{M}}(\mathrm{M} \cap$ $\left.x \bullet \mathrm{G}, \sigma^{*}\right)$ and $\operatorname{Hom}_{\mathrm{G}^{x}}(\pi, \mathbf{G})$.

Proof. - By our condition, for any $x \in \mathrm{X}$ the quotients $\mathcal{V}_{i+1} / \mathcal{V}_{i}, i \geq 2^{t-1}$ admit no $\mathrm{G}^{x}$-invariant functionals. Hence $\operatorname{Hom}_{\mathrm{G}^{x}}\left(\mathcal{V} / \mathcal{V}_{2^{t-1}}, \mathbf{G}\right)=0$, and it follows that $(\mathbf{6 . 7})$ is injective. Now let $\alpha \in \mathcal{E}_{\mathrm{M}}\left(\mathrm{M} \cap x \bullet \mathrm{G}, \sigma^{*}\right)$. By the support condition of unitary periods, $\mathrm{J}(x, \alpha, \lambda)$ is holomorphic at $\lambda=0$, for otherwise its leading term (along any line through 0 ) would not be supported on open orbits. Combined with $(\mathbf{6 . 6})$ this shows that the restriction map is also surjective. The rest of the Lemma also follows from $(\mathbf{6 . 6})$. 
Remark 6.8. - More generally, we say that the unitary periods on $\mathrm{I}_{\mathrm{M}}(\sigma, \lambda)$ are supported on open orbits if this holds for $\mathrm{I}_{\mathrm{M}}(\sigma[\lambda], 0)$. It is easy to see that this is the case for generic $\lambda \in \mathfrak{a}_{\mathrm{M}, \mathbf{G}}^{*}$, i.e. away from finitely many 'hyperplanes' of the form $\left\{\lambda: q^{\lambda_{i}-\lambda_{j}}=c\right\}$ for $1 \leq i<j \leq t$ and $c \neq 0$ (cf. [BD08]).

Lemma 6.9. - Suppose that the unitary periods on $\pi=\mathrm{I}(\sigma, 0)$ are supported on $w_{0} \bullet \mathrm{P}$. If $x \in w_{0} \bullet \mathrm{G}$ then the map $\operatorname{Hom}_{\mathrm{G}^{w_{0}}}\left(\mathrm{~V}_{w_{0}}^{\sigma}\left[w_{0}\right], \mathbf{G}\right) \rightarrow \operatorname{Hom}_{\mathrm{G}^{x}}(\pi, \mathbf{G})$ given by $\ell \mapsto \ell \circ p \circ \pi\left(\iota_{w_{0}}^{x}\right)$, where $p: \pi \rightarrow \mathrm{V}_{w_{0}}^{\sigma}\left[w_{0}\right]$ is the natural projection, is an isomorphism. On the other hand, if $x \notin w_{0} \bullet \mathrm{G}$ then $\operatorname{Hom}_{\mathrm{G}^{x}}(\pi, \mathbf{C})=0$. In particular, if in addition $\mathrm{M}$ is symmetric then $\beta \mapsto \mathrm{Z}(\beta, 0)$ is an isomorphism $\mathcal{E}_{\mathrm{M}}\left(\mathrm{X}_{\mathrm{M}}, \sigma^{*}\right) \rightarrow \mathcal{E}_{\mathrm{G}}\left(\mathrm{X}, \pi^{*}\right)$.

Proof. - By our condition $\mathcal{V}_{i+1} / \mathcal{V}_{i}$ admit no $\mathrm{G}^{x}$-invariant functions unless $i=k-1$ and $x \in w_{0} \bullet \mathrm{G}$. The first part immediately follows. The second part now follows from Lemma 6.4 (with $w=w_{0}$ and $\mathrm{L}=\mathrm{M}$ ) and Lemma 5.2.

In the case where $\sigma \in \Pi_{\mathrm{sqr}}^{\mathrm{M}}$ we can be more precise.

Lemma 6.10. - Let $\delta=\delta_{1} \otimes \cdots \otimes \delta_{t} \in \Pi_{\mathrm{sqr}}^{\mathrm{M}}$. Assume that for no $i<j$ we have $\delta_{i} \triangleleft \delta_{j}^{\tau}$. (For instance, this is satisfied if $\delta \in \Pi_{\text {cusp }}^{\mathrm{M}}$.) Suppose that $w \in \mathrm{W}_{2}[\mathrm{M}]$ and $y=w \operatorname{diag}\left(y_{1}, \ldots, y_{t}\right) \in$ $w \mathrm{M}(w) \cap \mathrm{X}$ is relevant for $\mathrm{I}(\delta)$. Then $w \mathrm{M} w=\mathrm{M}$ and viewed as an involution on $\{1, \ldots, t\}, w$ satisfies the following condition: for any $i=1, \ldots, t$,

$$
\begin{cases}\delta_{i} \text { is } \mathrm{G}_{n_{i}}^{w_{0} y_{i}} \text {-distinguished } & \text { if } w(i)=i, \\ \delta_{w(i)} \simeq \delta_{i}^{\tau} & \text { otherwise. }\end{cases}
$$

In particular, iffor no $i<j$ we have $\delta_{i} \unlhd \delta_{j}^{\tau}$ then unitary periods on $\mathrm{I}(\delta)$ are supported on open orbits.

Proof. - Let $\mathrm{L}=\mathrm{M}(w)$. $\mathrm{By}(\mathbf{6 . 4}) r_{\mathrm{L}, \mathrm{M}}(\delta)$ is $\mathrm{L}^{y}$-distinguished, and in particular nonzero. Recall that $\mathrm{L}$ is of type $\gamma$ with indices as in the notation of Section 6.1. Note that

$$
r_{\mathrm{L}, \mathrm{M}}(\delta)=r_{\mathrm{M}_{\gamma_{1}}, \mathrm{G}_{n_{1}}}\left(\delta_{1}\right) \otimes \cdots \otimes r_{\mathrm{M}_{\gamma_{t}}, \mathrm{G}_{n_{t}}}\left(\delta_{t}\right)
$$

and recall that

$$
r_{\mathrm{M}_{\gamma_{i}}, \mathrm{G}_{n_{i}}}\left(\delta_{i}\right)=\delta_{i, 1} \otimes \cdots \otimes \delta_{i, s_{i}} \in \Pi_{\mathrm{sqr}}^{\mathrm{M}_{\gamma_{i}}}
$$

(see $(\mathbf{1 . 1 3})$ for the description of $\left.\delta_{i, j}\right)$. As before, view $w$ as an involution on $\mathfrak{I}(w)$. The property $(\mathbf{6 . 1})$ implies that there exists $i_{0}$ such that $w(1,1)=\left(i_{0}, s_{i_{0}}\right)$.

Suppose first that $i_{0}=1$. Then $s_{1}=1$, for otherwise we would have $\delta_{1,1}^{\tau} \simeq \delta_{1, s_{1}}$ by (6.5) which would contradict the description of $r_{\mathrm{M}_{\gamma_{1}}, \mathrm{G}_{n_{1}}}\left(\delta_{1}\right)$. Thus, $y$ has the form $y=\operatorname{diag}\left(y_{1}, y^{\prime}\right)$ for some $y^{\prime} \in \mathrm{X}_{n-n_{1}}$ and by $(\mathbf{6 . 5}) \delta_{1}$ is $\mathrm{G}_{n_{1}}^{w_{0} y_{1}}$-distinguished.

Suppose now that $i_{0} \neq 1$. Then, once again by $(\mathbf{6 . 5})$ we have $\delta_{1,1}^{\tau} \simeq \delta_{i_{0}, s_{0}}$ which implies that $\delta_{1} \unlhd \delta_{i_{1}}^{\tau}$. By our assumption on $\delta$, we therefore have $\delta_{1}=\delta_{i_{1}}^{\tau}$, and then necessarily $s_{1}=s_{i_{0}}=1$. 
Thus, whether or not $i_{0}=1$, the induced representation $X_{j \neq 1, i_{0}} \delta_{j}$ admits a relevant orbit which is obtained from $w$ and $y$ by omitting the entry(ies) $\left\{1, i_{0}\right\}$. By induction, it follows immediately that $w$ is an involution satisfying $(\mathbf{6 . 8})$.

We will also need the following variant of the previous Lemma.

Lemma 6.11. - Let $\delta=\delta_{1} \otimes \cdots \otimes \delta_{t} \in \Pi^{\mathrm{M}}$ and let $1 \leq k \leq t$. Assume that

(1) $\delta_{i} \in \Pi_{\text {sqr }}$ for all $i \neq k$; say $\delta_{i}=\Delta_{\sigma_{i}}\left(\left[a_{i}, b_{i}\right]\right)$ for $\sigma_{i} \in \Pi_{\text {cusp }}$.

(2) There are no $i<j$ different from $k$ such that $\delta_{i} \unlhd \delta_{j}$.

(3) $\operatorname{supp}_{c}\left(\delta_{k}\right) \cap\left\{\sigma_{1}\left[b_{1}\right], \ldots, \sigma_{k-1}\left[b_{k-1}\right], \sigma_{k+1}\left[a_{k+1}\right], \ldots, \sigma_{t}\left[a_{t}\right]\right\}=\emptyset$.

Then unitary periods on $\mathrm{I}(\delta)$ are supported on open orbits.

Proof. - The Lemma is trivially true for $t=1$. Suppose therefore that $t>1$ and that $w \in \mathrm{W}_{2}[\mathrm{M}]$ is relevant for $\delta$. As before, we view $w$ as an involution on the set $\mathfrak{I}(w)$. We have $w(1,1)=\left(i_{0}, s_{i_{0}}\right)$ for some $i_{0}=1, \ldots, t$ and $w\left(t, s_{t}\right)=\left(j_{0}, 1\right)$ for some $j_{0}=1, \ldots, t$. Suppose that $k \neq 1$. As in the argument of the previous Lemma, we run into a contradiction unless $i_{0}=1$ and $s_{1}=1$. Similarly, if $k \neq t$ then necessarily $j_{0}=t$ and $s_{t}=1$. Hence, we can continue by induction on $t$.

6.3. Proof of Theorem 6.1. - Suppose that $\pi \in \Pi$ is $\mathrm{G}^{x}$-distinguished. We want to show that $\pi \in \Pi^{\tau}$. We already treated the supercuspidal case (by a global argument). Assume now that $\pi \in \Pi_{\text {sqr }}$. Write $\pi=\Delta_{\sigma}([a, b])$ with $\sigma \in \Pi_{\text {cusp. }}$ Then the induced representation $\tilde{\pi}=\sigma[a] \times \cdots \times \sigma[b]$ is also $\mathrm{G}^{x}$-distinguished. It follows from Lemma 6.10 that unitary periods on $\tilde{\pi}$ are supported on open orbits. It therefore follows from $(\mathbf{6 . 5})$ that $\sigma$ is distinguished by some unitary group. We now appeal to the supercuspidal case to conclude that $\sigma \in \Pi_{\text {cusp }}^{\tau}$ and therefore $\pi \in \Pi_{\text {sqr }}^{\tau}$.

Consider the general case. Assume that $\pi$ is the unique irreducible quotient of $\mathrm{I}(\delta, \lambda)$ where $\delta=\delta_{1} \otimes \cdots \otimes \delta_{t} \in \Pi_{\text {usqr }}^{\mathrm{M}}$ and $\lambda \in \overline{\left(\mathfrak{a}_{\mathrm{M}}^{*}\right)_{+}}$. By the uniqueness of the Langlands data it follows that $\lambda$ is uniquely determined by $\pi$ and $\delta$ is uniquely determined up to a permutation that fixes $\lambda$. Note that $\pi$ is Galois invariant if and only if there exists an involution $\xi$ on the indices $\{1, \ldots, t\}$ such that $\lambda_{\xi(i)}=\lambda_{i}$ and $\delta_{\xi(i)} \simeq \delta_{i}^{\tau}$ for all $i=1, \ldots, t$. If $\pi$ is $\mathrm{G}^{x}$-distinguished then so is $\mathrm{I}(\delta, \lambda)$. It follows from the analysis of Section 6.2 that there exists $w \in \mathrm{W}_{2}[\mathrm{M}]$ and $y=w \operatorname{diag}\left(y_{1}, \ldots, y_{t}\right) \in w \mathrm{M}(w) \cap x \bullet \mathrm{G}$ which is relevant for $\mathrm{I}(\delta, \lambda)$. The first part of Theorem 6.1 now follows from the square integrable case and Lemma 6.10 applied to $\delta[\lambda]$.

To show the second part of the Theorem, suppose that $\pi \in \Pi^{\tau}$ is totally $\tau$-isotropic (and in particular $n$ is even). By Lemma 3.4, we can write $\pi=\sigma \times \sigma^{\tau}$ for some $\sigma \in$ $\Pi^{\mathrm{G}_{n / 2}}$ such that $\sigma$ and $\sigma^{\tau}$ are disjoint. In particular, $\operatorname{supp}_{c}(\sigma) \cap \operatorname{supp}_{c}\left(\sigma^{\tau}\right)=\emptyset$. Let $\mathrm{P}$ be the parabolic subgroup of type $(n / 2, n / 2)$. We claim that the unitary periods on $\pi$ are supported on $w_{0} \bullet \mathrm{P}$. Recall that $w_{0} \bullet \mathrm{P}$ is the only orbit above $w_{0}$. Suppose that 
$w_{0} \neq w \in \mathrm{W}_{2}[\mathrm{M}]$. Then as a permutation on $[1, n], w$ necessarily has the form

$$
w(i)= \begin{cases}n+1-i & i=1, \ldots, k, n+1-k, \ldots, n, \\ n / 2+k+1-i & i=k+1, \ldots, n / 2, \\ 3 n / 2+1-k-i & i=n / 2+1, \ldots, n-k,\end{cases}
$$

for some $0 \leq k<n / 2$ and $\mathrm{L}:=\mathrm{M}(w)=\mathrm{G}_{k} \times \mathrm{G}_{n / 2-k} \times \mathrm{G}_{n / 2-k} \times \mathrm{G}_{k}$. If $y$ lies above $w$ then $\mathrm{L}^{y}$ is isomorphic to the product of $\mathrm{G}_{k}$ (embedded twisted diagonally in the first and last coordinate of $\mathrm{L}$ ) and a product of two unitary groups (in each of the middle coordinates). Observe that by the property of $\sigma$, for any irreducible subquotient $\sigma_{1} \otimes \sigma_{2}$ of the Jacquet module of $\sigma$ with respect to a maximal parabolic of $\mathrm{G}_{n / 2}$ we have $\sigma_{2} \not$ $\sigma_{2}^{\tau}$. Thus, $r_{\mathrm{L}, \mathrm{M}}\left(\sigma \otimes \sigma^{\tau}\right)$ is not $\mathrm{L}^{y}$-distinguished by the first part of Theorem 6.1 already proved. By $(\mathbf{6 . 4})$ this affirms our claim.

The Theorem now follows from Lemma 6.4, Lemma 6.9, and the fact that

$$
\operatorname{Hom}_{\mathrm{M}^{w}}\left(\sigma \otimes \sigma^{\tau}, \mathbf{C}\right) \simeq \operatorname{Hom}_{\mathrm{G}_{n / 2}}\left(\sigma \otimes \sigma^{\tilde{\theta}}, \mathbf{G}\right) \simeq \operatorname{Hom}_{\mathrm{G}_{n / 2}}\left(\sigma \otimes \sigma^{\vee}, \mathbf{G}\right)
$$

is one-dimensional.

6.4. Further remarks. - It is easy to see that if $\operatorname{supp}_{c}\left(\pi_{1}\right) \cap \operatorname{supp}_{c}\left(\pi_{2}^{\tau}\right)=\emptyset$ then the unitary periods on $\pi_{1} \times \pi_{2}$ are supported on open orbits. Therefore, by Lemma 6.7 and Theorem 6.1 , the study of $\mathcal{E}_{\mathrm{G}}\left(\mathrm{X}, \pi^{*}\right)$ reduces to the case where $\pi$ is pure and Galoisinvariant. Indeed, by Lemma 3.4, any $\pi \in \Pi^{\tau}$ can be written as $\pi=\pi_{1} \times \pi_{2}$ where $\pi_{1} \in \Pi^{\mathrm{G}_{n_{1}}, \tau, \mathrm{ti}}$ and $\pi_{2} \in \Pi^{\mathrm{G}_{n_{2}}, \tau, \text { an }}$. Thus,

$$
\mathcal{E}_{\mathrm{G}}\left(\mathrm{X}, \pi^{*}\right) \simeq \mathcal{E}_{\mathrm{G}_{n_{1}}}\left(\mathrm{X}_{n_{1}}, \pi_{1}^{*}\right) \otimes \mathcal{E}_{\mathrm{G}_{n_{2}}}\left(\mathrm{X}_{n_{2}}, \pi_{2}^{*}\right) \simeq \mathcal{E}_{\mathrm{G}_{n_{2}}}\left(\mathrm{X}_{n_{2}}, \pi_{2}^{*}\right)
$$

and

$$
\operatorname{Hom}_{\mathrm{G}^{x}}(\pi, \mathbf{G}) \simeq\left\{\begin{array}{c}
\operatorname{Hom}_{\mathrm{G}_{n_{2}}^{y}}\left(\pi_{2}, \mathbf{G}\right) \\
\quad \text { if } \exists y \in \mathrm{X}_{n_{2}} \text { such that } x=\operatorname{diag}\left(w_{0}^{\mathrm{G}_{n_{1}}}, y\right) \bullet \mathrm{G}, \\
0 \quad \text { otherwise, i.e., } n_{2}=0 \text { and } x \notin w_{0} \bullet \mathrm{G} .
\end{array}\right.
$$

This reduces to the case where $\pi \in \Pi^{\tau, \text { an }}$. By decomposing $\pi \in \Pi^{\tau \text {,an }}$ into its pure components $\pi_{1}, \ldots, \pi_{t}$ (which are Galois invariant) we get similarly that

$$
\mathcal{E}_{\mathrm{G}}\left(\mathrm{X}, \pi^{*}\right) \simeq \mathcal{E}_{\mathrm{M}}\left(\mathrm{X}^{\mathrm{M}}, \pi_{1}^{*} \otimes \cdots \otimes \pi_{t}^{*}\right) \simeq \bigotimes_{i=1}^{t} \mathcal{E}_{\mathrm{G}_{n_{i}}}\left(\mathrm{X}_{n_{i}}, \pi_{i}^{*}\right)
$$

and for any $x \in \mathrm{X}$

$$
\operatorname{Hom}_{\mathrm{G}^{x}}(\pi, \mathbf{G}) \simeq \mathcal{E}_{\mathrm{M}}\left(\mathrm{X}^{\mathrm{M}} \cap x \bullet \mathrm{G}, \pi_{1}^{*} \otimes \cdots \otimes \pi_{t}^{*}\right) .
$$

In the quasi-split case, Theorem 6.1 and its converse were conjectured by Jacquet, in analogy with the main global result of [Jac10]. 
6.5. A general conjecture. - Combining Theorem 6.1 (for the $p$-adic case) and Appendix $\mathrm{B}$ (for the Archimedean case) we obtain that if $\pi \in \Pi$ is $\mathrm{G}^{x}$-distinguished then $\pi \in \Pi^{\tau}$ and $\tilde{\mathfrak{w}}(\pi) \leq \mathfrak{w}(x)$. (We recall that $\mathfrak{w}(x)$ is the Witt index of $x$ and $\tilde{\mathfrak{w}}(\pi)$ is the $\tau$-Witt index of $\pi$ defined in Section 3.2.) We conjecture that the converse also holds. In other words,

Conjecture 6.12. - Suppose that $\pi \in \Pi$ and $x \in \mathrm{X}$. Then $\pi$ is $\mathrm{G}^{x}$-distinguished if and only if $\pi \in \Pi^{\tau}$ and $\tilde{\mathfrak{w}}(\pi) \leq \mathfrak{w}(x)$.

We will show this in Corollary 12.3 below for generic representations. Moreover, we will show that $\pi \in \Pi_{\text {gen }}^{\tau}$ is distinguished by $\mathrm{G}^{x}$ if and only if there exists $\pi^{\prime} \in \mathcal{B}(\pi)$ such that $\alpha_{x}^{\pi^{\prime}} \not \equiv 0$ (Corollary 12.9). We will also show Conjecture 6.12 in the unitarizable case.

We finish this section with a couple of instances of Conjecture 6.12 in the Archimedean case. First note that for compact unitary groups (i.e., for $x$ anisotropic) the conjecture reduces to Lemma 3.3.

As was pointed to us by Aizenbud, the conjecture holds for finite-dimensional $\pi$. Indeed,

Lemma 6.13. - Suppose that $\mathrm{E} / \mathrm{F}=\mathbf{C} / \mathbf{R}$ and $\pi \in \Pi$ is finite-dimensional and let $x \in \mathrm{X}$. Then $\pi$ is distinguished by $\mathrm{G}^{x}$ if and only if $\pi \in \Pi^{\tau}$ if and only if $\pi \in \Pi_{\mathrm{unr}}$.

Proof. - The statement is evidently invariant under twisting by an unramified character. Therefore we may assume that there exist algebraic irreducible representations $\sigma_{1}, \sigma_{2}$ of $\mathrm{GL}_{n} / \mathbf{C}$ such that $\pi$ is the restriction of $\sigma=\sigma_{1} \otimes \sigma_{2}$ to $\mathrm{G}$ under the embedding $g \mapsto\left(g, g^{\tau}\right)$. The condition on $\mathrm{L} \in \pi^{*}$ to be invariant under $\mathrm{G}^{x}$ is that $\mathrm{L} \circ \sigma\left(g,\left(x g^{-1} x^{-1}\right)^{t}\right)=\mathrm{L}$ for all $g \in \mathrm{G}^{x}$. Since $\mathrm{G}^{x}$ is Zariski dense in $\mathrm{GL}_{n}(\mathbf{C})$, the condition becomes $\mathrm{L}^{\prime} \circ \sigma\left(g,{ }^{t} g^{-1}\right)=\mathrm{L}^{\prime}$ for all $g \in \mathrm{G}$ where $\mathrm{L}^{\prime}=\mathrm{L} \circ \sigma\left(1,{ }^{t} x^{-1}\right)$. Such a non-zero $\mathrm{L}^{\prime}$ exists if and only if $\sigma_{2}=\sigma_{1}$. This proves the first equivalence. The second equivalence follows from the Cartan-Helgason Theorem.

Finally,

Lemma 6.14. - Conjecture 6.12 holds for $\Pi_{\mathrm{unr}}$.

Proof. - In the $p$-adic case, the statement follows from Corollary 4.2 and the fact that every unramified representation is induced from an unramified character of a Levi subgroup. (The latter follows from the Zelevinsky classification [Zel80].) Consider the complex case. Suppose that $\pi \in \Pi_{\text {unr }}$, i.e., $\pi$ is distinguished by $\mathrm{U}(n)$. By [Bar89, p. 129] there exists a parabolic subgroup $\mathrm{P}=\mathrm{MU}$, a finite-dimensional $\sigma \in \Pi_{\mathrm{unr}}^{\mathrm{M}}$ with trivial central character and $\lambda_{0} \in \mathfrak{a}_{\mathrm{M}, \mathbf{c}}^{*}$ such that $\pi=\mathrm{I}\left(\sigma, \lambda_{0}\right)$. Let $x \in \mathrm{X}$ and suppose without loss of generality that $x \in \mathbf{M}_{0}$. By Lemma 6.13, $\sigma$ is $\mathbf{M}^{x}$-distinguished. Therefore, 
there exists $0 \neq \alpha \in \mathcal{E}_{\mathrm{M}}\left(\mathrm{X}^{\mathrm{M}}, \sigma^{*}\right)$ such that $\alpha_{y} \equiv 0$ for all $y \notin x \bullet \mathrm{M}$. Since $\sigma$ is finitedimensional we have $\sigma=\mathrm{LQ}^{\mathrm{M}}\left(1_{\mathrm{M}_{0}}, \mu\right)$ for some $\mu \in\left(\mathfrak{a}_{0}^{\mathrm{M}}\right)_{+}^{*}$. By Theorem B.7 we have $\alpha \circ p=\mathrm{J}_{\mathrm{M}_{0}}^{\mathrm{M}}(\beta, \mu)$ for some $\beta \in \mathcal{E}_{\mathrm{M}_{0}}\left(\mathrm{X}^{\mathrm{M}_{0}}, 1_{\mathrm{M}_{0}}^{*}\right)$ where $p: \mathrm{I}_{\mathrm{M}_{0}}^{\mathrm{M}}\left(1_{\mathrm{M}_{0}}, \mu\right) \rightarrow \sigma$ is the natural projection. By the same Theorem $\mathrm{J}(\beta, \lambda+\mu)$ is holomorphic and non-zero for $\operatorname{Re} \lambda$ sufficiently regular in $\left(\mathfrak{a}_{\mathrm{M}}^{*}\right)_{+}$(i.e., when $\left.\lambda+\mu \in\left(\mathfrak{a}_{0}\right)_{+}^{*}\right)$. Therefore, by Lemma 4.4, $\mathrm{J}_{\mathrm{M}}(\alpha \circ p, \lambda)=\mathrm{J}_{\mathrm{M}}\left(\mathrm{J}_{\mathrm{M}_{0}}^{\mathrm{M}}(\beta, \mu), \lambda\right)$ is holomorphic and non-zero for $\operatorname{Re} \lambda$ sufficiently positive, and meromorphic for $\lambda \in \mathfrak{a}_{\mathrm{M}, \mathbf{G}}^{*}$. Note that $\mathrm{J}_{\mathrm{M}}(\alpha \circ p, \lambda)=\mathrm{J}_{\mathrm{M}}(\alpha, \lambda) \circ \mathrm{I}_{\mathrm{M}}^{\mathrm{G}}(p)$. Therefore $\mathrm{J}_{\mathrm{M}}(\alpha, \lambda)$ is also non-zero and meromorphic in $\lambda$. Arguing as in the proof of Corollary 4.2 this implies that $\mathrm{I}(\sigma, \lambda)$ is $\mathrm{G}^{x}$-distinguished for all $\lambda$.

\section{Local Bessel identities-the split case}

In this section we consider the split case. For $g \in \mathrm{G}^{\prime}$ let $g^{l}={ }^{t} g^{-1}$. Note that

$$
\mathrm{X}=\left\{\left(g,{ }^{t} g\right): g \in \mathrm{G}^{\prime}\right\}
$$

is a single G-orbit and for every $x=\left(h,{ }^{t} h\right) \in \mathrm{X}$ we have

$$
\mathrm{G}^{x}=\left\{\left(g,\left(h g h^{-1}\right)^{\iota}\right): g \in \mathrm{G}^{\prime}\right\} .
$$

We first relate the relative Bessel distributions on $\mathrm{X}$ with the usual Bessel distributions on $\mathrm{G}^{\prime}$.

Lemma 7.1. - Let $\mathcal{D}_{i}^{\prime}=\left(\pi_{i}^{\prime}, \hat{\pi}_{i}^{\prime},(\cdot, \cdot)_{i}^{\prime}\right)$ be dual couples of representations of $\mathrm{G}^{\prime}$ and let $\hat{\ell}_{i} \in \hat{\pi}_{i}^{\prime *}, i=1$, 2. Let $\mathrm{A}: \pi_{2}^{\prime} \rightarrow \hat{\pi}_{1}^{\prime \prime}$ be an intertwining operator. Define $\alpha \in \mathcal{E}_{\mathrm{G}}\left(\mathrm{X},\left(\pi_{1}^{\prime} \otimes \pi_{2}^{\prime}\right)^{*}\right)$ by $\alpha_{\left(g, g_{g}\right)}\left(v_{1} \otimes v_{2}\right)=\left(\pi_{1}^{\prime}(g) v_{1}, \mathrm{~A} v_{2}\right)_{1}^{\prime}, g \in \mathrm{G}^{\prime}, v_{1} \in \pi_{1}^{\prime}, v_{2} \in \pi_{2}^{\prime}$. Then

$$
\tilde{\mathfrak{B}}_{\mathcal{D}_{1}^{\prime} \otimes \mathcal{D}_{2}^{\prime}}^{\alpha, \hat{\ell}_{1} \otimes \hat{\ell}_{2}} \longleftrightarrow \mathfrak{B}_{\mathcal{D}_{2}^{\prime}}^{\hat{\ell}_{1} \circ \mathrm{A}, \hat{\ell}_{2}}
$$

Proof. - We first observe that the statement of the Lemma is invariant under $\hat{\ell}_{i} \mapsto$ $\hat{\ell}_{i} \circ \hat{\pi}_{i}\left(f_{i}^{\prime}\right), i=1,2$ for any $f_{1}^{\prime}, f_{2}^{\prime} \in \mathcal{S}\left(\mathrm{G}^{\prime}\right)$. Indeed, by (2.8) and (2.2) we have

$$
\tilde{\mathfrak{B}}_{\mathcal{D}_{1}^{\prime} \otimes \mathcal{D}_{2}^{\prime}}^{\alpha, \hat{\ell}_{1} \circ \hat{\pi}_{1}^{\prime}\left(f_{1}^{\prime}\right) \otimes \hat{\ell}_{2} \circ \hat{\pi}_{2}^{\prime}\left(f_{2}^{\prime}\right)}(\Phi)=\tilde{\mathfrak{B}}_{\mathcal{D}_{1}^{\prime} \otimes \hat{\mathcal{L}}_{2}^{\prime} \otimes \hat{\ell}_{2}}^{\prime}\left(\left[f_{1}^{\prime} \otimes f_{2}^{\prime}\right] * \Phi\right)
$$

and

$$
\begin{aligned}
\mathfrak{B}_{\mathcal{D}_{2}^{\prime}}^{\hat{\ell}_{1} \circ \hat{\pi}_{1}^{\prime}\left(f_{1}^{\prime}\right) \circ \mathrm{A}, \hat{\ell}_{2} \circ \pi_{2}^{\prime}\left(f_{2}^{\prime}\right)}\left(f^{\prime}\right) & =\mathfrak{B}_{\mathcal{D}_{2}^{\prime}}^{\hat{\ell}_{1} \circ \mathrm{A} \circ \hat{\pi}_{2}^{\prime}\left(\left(f_{1}^{\prime}\right)^{\iota}\right), \hat{\ell}_{2} \circ \pi_{2}^{\prime}\left(f_{2}^{\prime}\right)}\left(f^{\prime}\right) \\
& =\mathfrak{B}_{\mathcal{D}_{2}^{\prime}}^{\hat{\ell}_{1} \circ \mathrm{A}, \hat{\ell}_{2} \circ \pi_{2}^{\prime}\left(f_{2}^{\prime}\right)}\left(\left(f_{1}^{\prime}\right)^{\iota} * f^{\prime} *\left(f_{2}^{\prime}\right)^{\vee}\right)
\end{aligned}
$$

where $\left(f_{1}^{\prime}\right)^{\iota}\left(g^{\prime}\right)=f_{1}^{\prime}\left(g^{\prime l}\right)$ and $\left(f_{2}^{\prime}\right)^{\vee}\left(g^{\prime}\right)=f_{2}\left(g^{\prime-1}\right)$. On the other hand, it is evident from the definition of matching in the split case that

$$
\left[f_{1}^{\prime} \otimes f_{2}^{\prime}\right] * \Phi \longleftrightarrow\left(f_{1}^{\prime}\right)^{\iota} * f^{\prime} *\left(f_{2}^{\prime}\right)^{\vee}
$$

whenever $\Phi \longleftrightarrow f^{\prime}$. 
Thus, writing $f^{\prime}$ as a linear combination of $f_{1}^{\prime} * h^{\prime} * f_{2}^{\prime}$ for some $f_{1}^{\prime}, f_{2}^{\prime}, h^{\prime} \in \mathcal{S}\left(\mathrm{G}^{\prime}\right)$, we reduce the Lemma to the case where $\hat{\ell}_{i} \in\left(\hat{\pi}_{i}^{\prime}\right)^{\vee}, i=1,2$. In other words, we may assume that there exist $u_{i}^{\prime} \in \pi_{i}^{\prime}$ such that $\hat{\ell}_{i}\left(v_{i}\right)=\left(u_{i}^{\prime}, v_{i}\right)_{i}^{\prime}$ for all $v_{i} \in \hat{\pi}_{i}^{\prime}, i=1,2$.

Fix $\Phi \longleftrightarrow f^{\prime}$. Observe that $\ell:=\Phi \odot \alpha \in\left(\pi_{1}^{\prime} \otimes \pi_{2}^{\prime}\right)^{\vee}$ is given by $\ell\left(v_{1}^{\prime} \otimes v_{2}^{\prime}\right)=$ $\left(v_{1}^{\prime}, \mathrm{A} \pi_{2}^{\prime}\left(f^{\prime}\right) v_{2}^{\prime}\right)_{1}^{\prime}, v_{i}^{\prime} \in \pi_{i}^{\prime}, i=1,2$. Indeed,

$$
\begin{aligned}
{[\Phi \odot \alpha]\left(v_{1}^{\prime} \otimes v_{2}^{\prime}\right) } & =\int_{\mathrm{X}} \Phi(x) \alpha_{x}\left(v_{1}^{\prime} \otimes v_{2}^{\prime}\right) d x=\int_{\mathrm{G}^{\prime}} f^{\prime}(g)\left(\pi_{1}^{\prime}\left({ }^{t} g\right) v_{1}^{\prime}, \mathrm{A} v_{2}^{\prime}\right)_{1}^{\prime} d g \\
& =\int_{\mathrm{G}^{\prime}} f^{\prime}(g)\left(v_{1}^{\prime}, \hat{\pi}_{1}^{\prime}\left(g^{\imath}\right) \mathrm{A} v_{2}^{\prime}\right)_{1}^{\prime} d g \\
& =\int_{\mathrm{G}^{\prime}} f^{\prime}(g)\left(v_{1}^{\prime}, \mathrm{A} \pi_{2}^{\prime}(g) v_{2}^{\prime}\right)_{1}^{\prime} d g=\ell\left(v_{1}^{\prime} \otimes v_{2}^{\prime}\right) .
\end{aligned}
$$

Thus

$$
\tilde{\mathfrak{B}}_{\mathcal{D}_{1}^{\prime} \otimes \mathcal{D}_{2}^{\prime}}^{\alpha, \hat{\ell}_{1}}(\Phi)=\left(\hat{\ell}_{1} \otimes \hat{\ell}_{2}\right)\left(\Lambda_{\mathcal{D}_{1}^{\prime} \otimes \mathcal{D}_{2}^{\prime}} \ell\right)=\ell\left(u_{1}^{\prime} \otimes u_{2}^{\prime}\right)=\left(u_{1}^{\prime}, \mathrm{A} \pi_{2}^{\prime}\left(f^{\prime}\right) u_{2}^{\prime}\right)_{1}^{\prime}
$$

On the other hand,

$$
\left[\hat{\ell}_{1} \circ \mathrm{A} \circ \pi_{2}^{\prime}\left(f^{\prime}\right)\right]\left(u^{\prime}\right)=\ell\left(u_{1}^{\prime} \otimes u^{\prime}\right), \quad u^{\prime} \in \pi_{2}^{\prime},
$$

so that

$$
\begin{aligned}
\mathfrak{B}_{\mathcal{D}_{2}^{\prime}}^{\hat{\ell}_{1} \circ \mathrm{A}, \hat{\ell}_{2}}\left(f^{\prime}\right)=\hat{\ell}_{2} \circ \Lambda_{\mathcal{D}_{2}^{\prime}}\left(\hat{\ell}_{1} \circ \mathrm{A} \circ \pi_{2}^{\prime}\left(f^{\prime}\right)\right) & =\left[\hat{\ell}_{1} \circ \mathrm{A} \circ \pi_{2}^{\prime}\left(f^{\prime}\right)\right]\left(u_{2}^{\prime}\right) \\
& =\ell\left(u_{1}^{\prime} \otimes u_{2}^{\prime}\right) .
\end{aligned}
$$

The Lemma follows.

Let $\pi^{\prime} \in \Pi_{\text {gen }}^{\prime}$ and $\pi=\operatorname{bc}\left(\pi^{\prime}\right)=\pi^{\prime} \otimes \pi^{\prime} \in \Pi_{\text {gen }}^{\tau}$ and recall that $\mathcal{B}(\pi)=\left\{\pi^{\prime}\right\}$ and $\mathfrak{W}(\pi)=\mathfrak{W}\left(\pi^{\prime}\right) \otimes \mathfrak{W}\left(\pi^{\prime}\right)$. Recall also the definition of $\mathcal{B I}$ and $\alpha^{\pi^{\prime}}$ from Section 3.3. Taking $\mathcal{D}_{1}^{\prime}=\mathcal{D}_{2}^{\prime}=\mathfrak{W}\left(\pi^{\prime}\right), \hat{\ell}_{1}=\hat{\ell}_{2}=\delta_{e}^{\pi^{\prime \vee}}$ and $\mathrm{A}=\mathfrak{y}_{\pi^{\prime}} \circ \mathcal{W}\left(w_{0}, \pi^{\prime}\right)$ in the previous Lemma we get we have

Corollary 7.2. - (Cf. [Jac01, Lemma 2]) We have $\mathcal{B I}=\Pi_{\text {gen }}^{\prime}$. Moreover for any $\pi^{\prime} \in \Pi_{\text {gen }}^{\prime}$

$$
\alpha_{\left(h,{ }^{\prime} h\right)}^{\pi^{\prime}}\left(\mathrm{W}^{\prime} \otimes \mathrm{W}^{\prime \prime}\right)=\left[\mathcal{W}\left(h, \pi^{\prime}\right) \mathrm{W}^{\prime}, \mathfrak{y}_{\pi^{\prime}} \circ \mathcal{W}\left(w_{0}, \pi^{\prime}\right) \mathrm{W}^{\prime \prime}\right]_{\pi^{\prime}}
$$

for any $h \in \mathrm{G}^{\prime}, \mathrm{W}^{\prime}, \mathrm{W}^{\prime \prime} \in \mathcal{W}\left(\pi^{\prime}\right)$. 
7.1. Open periods. - Let $\mathrm{P}^{\prime}=\mathrm{M}^{\prime} \mathrm{U}^{\prime}$ be a standard parabolic subgroup of $\mathrm{G}^{\prime}$ and $\mathrm{P}=\mathrm{MU}$ the corresponding parabolic subgroup of G. Let $\sigma^{\prime}=\sigma_{1}^{\prime} \otimes \cdots \otimes \sigma_{t}^{\prime} \in \Pi_{\text {gen }}^{\mathrm{M}^{\prime}}$. Next we show that $\sigma^{\prime} \in \mathcal{B I}_{\mathrm{M}}$. To that end, we use the results of Shahidi on local coefficients. Beforehand, we interpret the split open period in terms of the intertwining operator.

Recall the intertwining operator $(\mathbf{1 . 4})$

$$
\Theta\left(\sigma^{\prime}, \lambda\right):\left[\mathrm{I}_{\overleftarrow{\mathrm{M}}^{\prime}}\left(\mathcal{W}^{\psi}\left(\overleftarrow{\sigma}^{\prime}\right), \overleftarrow{\lambda}\right)\right]^{\tilde{\theta}} \rightarrow \mathrm{I}_{\mathrm{M}^{\prime}}\left(\mathcal{W}^{\psi^{-1}}\left(\sigma^{\prime \vee}\right),-\lambda\right)
$$

Composing with translation by $w_{0}$ we obtain an intertwining operator

$$
\tilde{\Theta}\left(\sigma^{\prime}, \lambda\right): \mathrm{I}_{\overleftarrow{\mathrm{M}}^{\prime}}\left(\mathcal{W}^{\psi}\left(\overleftarrow{\sigma}^{\prime}\right), \overleftarrow{\lambda}\right) \rightarrow\left[\mathrm{I}_{\mathrm{M}^{\prime}}\left(\mathcal{W}^{\psi^{-1}}\left(\sigma^{\prime \vee}\right),-\lambda\right)\right]^{\iota}
$$

By (1.5) we have

$$
\mathbf{W}_{e}\left(\sigma^{\prime \vee},-\lambda\right) \circ \tilde{\Theta}\left(\sigma^{\prime}, \lambda\right)=\mathbf{W}\left(w_{0}: w_{\mathrm{M}} \sigma^{\prime}, w_{\mathrm{M}} \lambda\right) .
$$

Lemma 7.3. - Let $\sigma^{\prime} \in \Pi_{\text {gen }}^{\mathrm{M}^{\prime}}, x=\left(h,{ }^{t} h\right)$ with $h \in \mathrm{G}^{\prime}$ and $\varphi^{\prime}, \varphi^{\prime \prime} \in \mathrm{I}\left(\mathcal{W}\left(\sigma^{\prime}\right)\right)$. As meromorphic functions of $\lambda \in \mathfrak{a}_{\mathrm{M}, \mathbf{C}}^{*}$ we have

$$
\begin{aligned}
& \mathrm{J}\left(\varphi^{\prime} \otimes \varphi^{\prime \prime}: x, \alpha^{\sigma^{\prime}}, \lambda\right) \\
& \quad=\left(\mathrm{I}\left(h, \mathcal{W}\left(\sigma^{\prime}\right), \lambda\right) \varphi^{\prime}, \tilde{\Theta}\left(\sigma^{\prime}, \lambda\right) \circ \mathrm{M}\left(w_{\mathrm{M}}, \mathcal{W}\left(\sigma^{\prime}\right), \lambda\right) \varphi^{\prime \prime}\right)_{\mathrm{I}\left([\cdot, \cdot]_{\sigma^{\prime}}\right)}
\end{aligned}
$$

and

$$
\begin{aligned}
& \mathcal{J}_{\sigma^{\prime}}\left(\varphi^{\prime} \otimes \varphi^{\prime \prime}: x, \alpha^{\sigma^{\prime}}, \lambda\right) \\
& \quad=\left(\mathrm{I}\left(h, \mathcal{W}\left(\sigma^{\prime}\right), \lambda\right) \varphi^{\prime}, \tilde{\Theta}\left(\sigma^{\prime}, \lambda\right) \circ \mathrm{N}\left(w_{\mathrm{M}}, \mathcal{W}\left(\sigma^{\prime}\right), \lambda\right) \varphi^{\prime \prime}\right)_{\mathrm{I}\left([\cdot \cdot \cdot]_{\sigma^{\prime}}\right)}
\end{aligned}
$$

Proof. - Since in the split case we have $\mathfrak{n}_{\mathrm{M}^{\prime}}\left(\sigma^{\prime}, \lambda\right)=\mathrm{C}_{\mathrm{M}^{\prime}}\left(w_{\mathrm{M}}: \sigma^{\prime}, \lambda ; \psi^{\prime}\right)$, the second equality is immediate from the first one. For $x=\left(h,{ }^{t} h\right)$ we may choose $\iota_{e}^{x}=(h, e)$. By Corollary 7.2, $\sigma^{\prime} \in \mathcal{B I}^{\mathrm{M}}$, and using the definition of $\mathrm{J}\left(\alpha^{\sigma^{\prime}}, \lambda\right)$, for $\operatorname{Re} \lambda$ sufficiently positive $\mathrm{J}\left(\varphi^{\prime} \otimes \varphi^{\prime \prime}: x, \alpha^{\sigma^{\prime}}, \lambda\right)$ is given by

$$
\begin{aligned}
& \int_{\mathrm{P}^{e} \backslash \mathrm{G}^{e}} \alpha_{e}^{\sigma^{\prime}}\left(\left(\varphi^{\prime} \otimes \varphi^{\prime \prime}\right)_{\lambda}\left(g \iota_{e}^{x}\right)\right) d g \\
& =\int_{\mathrm{M}^{\prime} \backslash \mathrm{G}^{\prime}}\left[\varphi_{\lambda}^{\prime}(g h), \mathfrak{y} \overleftarrow{\sigma^{\prime}}\left(\mathcal{W}^{\mathrm{M}}\left(w_{0}^{\mathrm{M}}, \sigma^{\prime}\right) \varphi_{\lambda}^{\prime \prime}\left(g^{l}\right)\right)\right]_{\sigma^{\prime}} d g \\
& =\int_{\mathrm{P}^{\prime} \backslash \mathrm{G}^{\prime}} \int_{\mathrm{U}^{\prime}}\left[\varphi_{\lambda}^{\prime}(g h), \mathfrak{y} \overleftarrow{\sigma^{\prime}}\left(\mathcal{W}^{\mathrm{M}}\left(w_{0}^{\mathrm{M}}, \sigma^{\prime}\right) \varphi_{\lambda}^{\prime \prime}\left(u^{l} g^{\imath}\right)\right)\right] d u d g \\
& =\int_{\mathrm{P}^{\prime} \backslash \mathrm{G}^{\prime}} \int_{\mathrm{U}^{\prime}}\left[\left(\mathrm{I}\left(h, \mathcal{W}\left(\sigma^{\prime}\right), \lambda\right) \varphi^{\prime}\right)_{\lambda}(g), \mathfrak{y}_{\sigma^{\prime}}\left(\varphi_{\lambda}^{\prime \prime}\left(w_{0}^{\mathrm{M}} u^{\iota} g^{l}\right)\right)\right] d u d g
\end{aligned}
$$

It remains to invoke (1.7). The Lemma follows by meromorphic continuation. 
Corollary 7.4. - For any $\sigma^{\prime} \in \Pi_{\text {gen }}^{\mathrm{M}^{\prime}}$ we have the identity

$$
\tilde{\mathbf{B}}\left(\sigma^{\prime}, \lambda\right) \longleftrightarrow \mathbf{B}\left(\sigma^{\prime}, \lambda\right)
$$

of meromorphic functions in $\lambda \in \mathfrak{a}_{\mathrm{M}, \mathbf{c}}^{*}$. Thus, $\mathcal{B I}_{\mathrm{M}^{\prime}}=\Pi_{\text {gen }}^{\mathrm{M}^{\prime}}$ and we have

$$
\alpha_{\mathrm{M}}\left(\sigma^{\prime}, \lambda\right)=\mathcal{J}_{\sigma^{\prime}}\left(\alpha^{\sigma^{\prime}}, \lambda\right)
$$

Proof. - Set $\mathcal{D}_{1}^{\prime}=\mathcal{D}_{2}^{\prime}=\mathrm{I}\left(\mathfrak{W}\left(\sigma^{\prime}\right), \lambda\right)$ and $\hat{\ell}_{1}=\hat{\ell}_{2}=\mathbf{W}_{e}\left(\sigma^{\prime \vee},-\lambda\right)$. Consider the intertwining operator

$$
\mathrm{A}=\tilde{\Theta}\left(\sigma^{\prime}, \lambda\right) \circ \mathrm{N}\left(w_{\mathrm{M}}, \mathcal{W}\left(\sigma^{\prime}\right), \lambda\right): \mathrm{I}\left(\mathcal{W}\left(\sigma^{\prime}\right), \lambda\right) \rightarrow \mathrm{I}\left(\mathcal{W}\left(\sigma^{\prime \vee}\right),-\lambda\right)^{\iota} .
$$

We have

$$
\hat{\ell}_{1} \circ \mathrm{A} \stackrel{(\mathbf{7 . 1})}{=} \mathbf{W}\left(w_{0}: w_{\mathrm{M}} \sigma^{\prime}, w_{\mathrm{M}} \lambda\right) \circ \mathrm{N}\left(w_{\mathrm{M}}, \mathcal{W}\left(\sigma^{\prime}\right), \lambda\right) \stackrel{(\mathbf{1 . 1 0})}{=} \mathbf{W}\left(w_{0}: \sigma^{\prime}, \lambda\right) .
$$

Taking Lemma 7.3 into account, the identity (7.2) therefore follows from Lemma 7.1 and the definition $(\mathbf{4 . 1 3})$ of $\tilde{\mathbf{B}}\left(\sigma^{\prime}, \lambda\right)$. The last part of the Corollary is then immediate from the definition of $\mathcal{B I}_{\mathrm{M}^{\prime}}$ and (2.11).

7.2. Closed split periods. - Next we will show the Bessel identities for the closed Bessel distributions. Let L be even symmetric.

Proposition 7.5. - Let $\varrho \in \Pi_{\text {gen }}^{\mathrm{L}, w_{0}}$. Then for any $\lambda \in\left(\mathfrak{a}_{\mathrm{L}}^{*}\right)_{\mathbf{G}}^{w_{0}}$ such that $\pi=\mathrm{I}(\varrho, \lambda)$ is irreducible we have

$$
\tilde{\mathbf{D}}(\varrho, \lambda) \longleftrightarrow \mathbf{B}_{\pi^{\prime}}
$$

where $\mathcal{B}(\pi)=\left\{\pi^{\prime}\right\}$.

By Proposition 5.9 and (2.14) it is enough to prove the Proposition in the case where $\mathrm{L}=\mathrm{M}_{(k, k)}$. This means that $\lambda \in \mathfrak{a}_{\mathrm{G}}^{*}$. We might as well assume (by translating by $\lambda$ ) that $\lambda=0$. We therefore analyze this case. Thus, for the rest of this section let $n=2 k$ and $\mathrm{L}=\mathrm{M}_{(k, k)}$ so that $\mathrm{L} \simeq \mathrm{G}_{k} \times \mathrm{G}_{k} \simeq\left(\mathrm{G}_{k}^{\prime} \times \mathrm{G}_{k}^{\prime}\right) \times\left(\mathrm{G}_{k}^{\prime} \times \mathrm{G}_{k}^{\prime}\right)$. To avoid ambiguity when considering representations of $\mathrm{L}$, we will continue to use $\otimes$ for the tensor product of representations with respect to the decomposition $\mathrm{L}=\mathrm{G}_{k} \times \mathrm{G}_{k}$ or $\mathrm{L}^{\prime}=\mathrm{G}_{k}^{\prime} \times \mathrm{G}_{k}^{\prime}$ and use the symbol $\square$ to express the tensor product with respect to $\mathrm{L}=\mathrm{L}^{\prime} \times \mathrm{L}^{\prime}$ or $\mathrm{G}=\mathrm{G}^{\prime} \times \mathrm{G}^{\prime}$.

Let $\varrho_{j}^{\prime} \in \mathcal{R}\left(\mathrm{G}_{k}^{\prime}\right), j=1,2, \varrho^{\prime}=\varrho_{1}^{\prime} \otimes \varrho_{2}^{\prime}, \overleftarrow{\varrho^{\prime}}=\varrho_{2}^{\prime} \otimes \varrho_{1}^{\prime}$ and

$$
\varrho=\varrho^{\prime} \otimes \overleftarrow{\varrho^{\prime}}=\left(\varrho_{1}^{\prime} \otimes \varrho_{2}^{\prime}\right) \otimes\left(\varrho_{2}^{\prime} \otimes \varrho_{1}^{\prime}\right)
$$


Assume further that $\varrho_{j}^{\prime} \in \Pi_{\text {gen }}^{\mathrm{G}_{k}^{\prime}}$. It is immediate from the definitions that for any $\mathrm{W}^{\prime} \in$ $\mathcal{W}\left(\varrho^{\prime}\right)$ and $Z^{\prime} \in \mathcal{W}\left(\varrho^{\prime \vee}\right)$ we have

$$
\left[\mathrm{W}^{\prime}, \mathrm{Z}^{\prime}\right]_{\varrho^{\prime}}=\beta_{w_{0}}\left(\mathrm{~W}^{\prime} \otimes \mathfrak{y}_{\varrho^{\prime}} \mathrm{Z}^{\prime}\right)
$$

We can identify

$$
\mathrm{I}(\varrho, 0)=\mathrm{I}\left(\varrho^{\prime}, 0\right) \otimes \mathrm{I}\left(\overleftarrow{\varrho^{\prime}}, 0\right)
$$

and

$$
\delta_{e}^{\varrho}=\delta_{e}^{\varrho^{\prime}} \nabla \delta_{e}^{\overleftarrow{\varrho^{\prime}}}
$$

on $\mathcal{W}(\varrho)$. Therefore,

$$
\mathbf{W}_{e}(\varrho, 0)=\mathbf{W}_{e}\left(\varrho^{\prime}, 0\right) \otimes \mathbf{W}_{e}\left(\overleftarrow{\varrho^{\prime}}, 0\right)
$$

Consider the intertwining operator

$$
\tilde{\Theta}\left(\varrho^{\prime}, 0\right): \mathrm{I}\left(\mathcal{W}\left(\overleftarrow{\varrho^{\prime}}\right), 0\right) \rightarrow \mathrm{I}\left(\mathcal{W}\left(\varrho^{\prime \vee}\right), 0\right)^{\iota}
$$

Recall that

$$
\mathbf{W}_{e}\left(\varrho^{\prime \vee}, 0\right) \circ \tilde{\Theta}\left(\varrho^{\prime}, 0\right)=\mathbf{W}\left(w_{0}: \overleftarrow{\varrho^{\prime}}, 0\right)
$$

Lemma 7.6. - With the above notation, let $\varphi=\varphi^{\prime} \otimes \varphi^{\prime \prime} \in \mathrm{I}(\mathcal{W}(\varrho))$ where $\varphi^{\prime} \in \mathrm{I}\left(\mathcal{W}\left(\varrho^{\prime}\right)\right)$ and $\varphi^{\prime \prime} \in \mathrm{I}\left(\mathcal{W}\left(\overleftarrow{\varrho^{\prime}}\right)\right)$. Then for $g \in \mathrm{G}^{\prime}$ and $x=\left(g,{ }^{t} g\right) \in \mathrm{X}$ we have

$$
\mathrm{Z}\left(\varphi: x, \beta^{\varrho}, 0\right)=\left(\mathrm{I}\left(g, \mathcal{W}\left(\varrho^{\prime}\right), 0\right) \varphi^{\prime}, \tilde{\Theta}\left(\varrho^{\prime}, 0\right) \varphi^{\prime \prime}\right)_{\mathrm{I}\left([\cdot, \cdot] \varrho_{\varrho^{\prime}}\right)} .
$$

Proof. - It suffices to prove this for $g=w_{0}$, so that $x=\left(w_{0}, w_{0}\right)$. We have

$$
\begin{aligned}
\mathrm{Z}\left(\varphi: x, \beta^{\varrho}, 0\right) & =\int_{Q^{\prime} \backslash \mathrm{G}^{\prime}} \beta_{w_{0}}\left(\varphi\left(h, h^{\tilde{\theta}}\right)\right) d h=\int_{\mathcal{Q}^{\prime} \backslash \mathrm{G}^{\prime}} \beta_{w_{0}}\left(\varphi^{\prime}(h) \otimes \varphi^{\prime \prime}\left(h^{\tilde{\theta}}\right)\right) d h \\
& =\int_{\mathcal{Q}^{\prime} \backslash \mathrm{G}^{\prime}} \beta_{w_{0}}\left(\varphi^{\prime}(h) \nabla \mathfrak{y}_{\varrho^{\prime}}\left(\tilde{\Theta}\left(\varrho^{\prime}, 0\right) \varphi^{\prime \prime}\left(h w_{0}\right)\right)\right) d h \\
& =\int_{Q_{Q^{\prime} \backslash \mathrm{G}^{\prime}}} \beta_{w_{0}}\left(\varphi^{\prime}\left(h w_{0}\right) \nabla \mathfrak{y}_{\varrho^{\prime}}\left(\tilde{\Theta}\left(\varrho^{\prime}, 0\right) \varphi^{\prime \prime}(h)\right)\right) d h .
\end{aligned}
$$

The Lemma now follows from the relation $(\mathbf{7 . 4})$.

Finally, we can go back to the proof of Proposition 7.5. 
Proof of Proposition 7.5. - Recall that we can assume that $\mathrm{L}=\mathrm{M}_{(k, k)}$ and $\lambda \in \mathfrak{a}_{\mathrm{G}}^{*}$. We identify $\mathcal{D}_{1}=\mathrm{I}(\mathfrak{W}(\varrho), 0)$ with $\mathcal{D}_{2}=\mathcal{D}_{1}^{\prime} \otimes \mathcal{D}_{2}^{\prime}$ where

$$
\mathcal{D}_{1}^{\prime}=\mathrm{I}\left(\mathfrak{W}\left(\varrho^{\prime}\right), 0\right) \quad \text { and } \quad \mathcal{D}_{2}^{\prime}=\mathrm{I}\left(\mathfrak{W}\left(\overleftarrow{\varrho^{\prime}}\right), 0\right)
$$

More precisely we identify $\pi_{1}=\mathrm{I}(\mathcal{W}(\varrho), 0)$, with $\pi_{2}=\pi_{1}^{\prime} \otimes \pi_{2}^{\prime}$ where $\pi_{1}^{\prime}=\mathrm{I}\left(\mathcal{W}\left(\varrho^{\prime}\right), 0\right)$ and $\pi_{2}^{\prime}=\mathrm{I}\left(\mathcal{W}\left(\overleftarrow{\varrho^{\prime}}\right), 0\right)$ and we identify $\hat{\pi}_{1}=\mathrm{I}\left(\mathcal{W}\left(\varrho^{\vee}\right), 0\right)$ with $\hat{\pi}_{2}=\hat{\pi}_{1}^{\prime} \otimes \hat{\pi}_{2}^{\prime}$ where $\hat{\pi}_{1}^{\prime}=\mathrm{I}\left(\mathcal{W}\left(\varrho^{\prime \vee}\right), 0\right)$, and $\hat{\pi}_{2}^{\prime}=\mathrm{I}\left(\mathcal{W}\left(\left(\overleftarrow{\varrho^{\prime}}\right)^{\vee}\right), 0\right)$. Under these identifications $\alpha_{1}=\mathrm{Z}\left(\beta^{\varrho}, 0\right)$ becomes $\alpha_{2} \in \mathcal{E}_{\mathrm{G}}\left(\mathrm{X}, \pi_{2}^{*}\right)$ given by

$$
\left(\alpha_{2}\right)_{\left(g,{ }_{g} g\right.}\left(\varphi_{1}^{\prime} \otimes \varphi_{2}^{\prime}\right)=\left(\pi_{1}^{\prime}(g) \varphi_{1}^{\prime}, \tilde{\Theta}\left(\varrho^{\prime}, 0\right) \varphi_{2}^{\prime}\right)_{\mathcal{D}_{1}^{\prime}}
$$

(by Lemma 7.6) and $\hat{\ell}_{1}=\mathbf{W}_{e}\left(\varrho^{\vee}, 0\right)$ becomes $\hat{\ell}_{2}=\hat{\ell}_{1}^{\prime} \otimes \hat{\ell}_{2}^{\prime}$ where

$$
\hat{\ell}_{1}^{\prime}=\mathbf{W}_{e}\left(\varrho^{\prime \vee}, 0\right) \quad \text { and } \quad \hat{\ell}_{2}^{\prime}=\mathbf{W}_{e}\left(\left(\overleftarrow{\varrho^{\prime}}\right)^{\vee}, 0\right)
$$

by (7.5). Thus

$$
\tilde{\mathbf{D}}(\varrho, 0)=\tilde{\mathfrak{B}}_{\mathcal{D}_{1}^{\prime} \otimes \mathcal{L}_{2}^{\prime}}^{\alpha_{2} \hat{\ell}_{1}^{\prime} \otimes \hat{\ell}_{2}^{\prime}}
$$

It therefore follows from $(\mathbf{7 . 6})$ and Lemma 7.1 that

$$
\mathfrak{B}_{\mathcal{D}_{1}^{\prime} \otimes \mathcal{D}_{2}^{\prime}}^{\alpha_{2}, \hat{\ell}_{1}^{\prime} \otimes \hat{\ell}_{2}^{\prime}} \longleftrightarrow \mathbf{B}\left(\overleftarrow{\varrho^{\prime}}, 0\right)
$$

By assumption, $\pi^{\prime} \simeq \mathrm{I}\left(\overleftarrow{\varrho^{\prime}}, 0\right)$. The Proposition now follows from $(\mathbf{2 . 1 4})$

\section{Local Bessel identities - the unramified case}

For principal series representations the sought-after Bessel identities were obtained in [Off07, Theorem 3] using results of Hironaka on spherical functions on X [Hir99]. Actually the results of [Off07] are only up to a possible twist by $\eta$ in the transfer factor $\gamma$, but this was subsequently determined in [Off09, Theorem 7.1]. From these results we will deduce the necessary identities for unramified data.

Throughout this section assume that $\mathrm{F}$ is $p$-adic, $p \neq 2, \mathrm{E} / \mathrm{F}$ is either split or unramified (i.e., $\eta$ is unramified) and $\psi^{\prime}$ is unramified.

8.1. Recollection of the results of $\left[\right.$ Hir99] and $[O f f 09]^{8}$. - Let $\varrho=\left(\varrho_{1}, \ldots, \varrho_{n}\right) \in \Pi_{\text {unr }}^{\mathrm{M}_{0}}$ be an unramified character of $\mathbf{M}_{0}$. Throughout we denote by $\varphi_{0}=\varphi_{0, \varrho}$ the standard

\footnotetext{
${ }^{8}$ Note that the notational conventions in [Off09] are slightly different.
} 
spherical section of $\mathrm{I}(\varrho)$ normalized by $\varphi_{0}(e)=1$. Let $\varrho^{\prime}=\left(\varrho_{1}^{\prime}, \ldots, \varrho_{n}^{\prime}\right) \in \mathcal{B}(\varrho)\left(\subseteq \Pi_{\text {unr }}^{\mathrm{M}_{0}^{\prime}}\right)$. Then $\varrho^{\prime} \in \mathcal{B} \mathcal{I}^{\mathrm{M}_{0}}$ and according to our normalization, the linear forms $\alpha_{y}^{\varrho^{\prime}}, y \in \mathrm{X}^{\mathrm{M}_{0}}=\mathrm{M}_{0}^{\prime}$ on $\mathcal{W}(\varrho)=\mathbf{C}$ are given by multiplication by $\mathrm{L}(1, \eta)^{n} \eta(\operatorname{det} y) \varrho^{\prime}(y)$ and $\mathcal{E}_{\mathrm{M}_{0}}\left(\mathrm{X}^{\mathrm{M}_{0}}, \mathcal{W}(\varrho)^{*}\right)$ is the span of $\left\{\alpha^{\varrho^{\prime}}: \varrho^{\prime} \in \mathcal{B}(\varrho)\right\}$. Let $\mathbf{E}_{\mathbf{1}}$ be the kernel of $\mathrm{Nm}: \mathbf{G}_{\mathbf{1}} \rightarrow \mathbf{G}_{\mathbf{1}}^{\prime}$ considered as an algebraic group over $\mathrm{F}$, so that $\mathrm{E}_{1}=\left\{x \in \mathrm{E}^{*}: x x^{\tau}=1\right\}$ and let

$$
\mathbf{M}_{\mathbf{1}}=\left\{\operatorname{diag}\left(a_{1}, \ldots, a_{n}\right) \in \mathbf{M}_{\mathbf{0}}: a_{i} \in \mathbf{E}_{\mathbf{1}}\right\}
$$

Note that $\mathrm{P}_{0}^{y}=\mathrm{M}_{1}$ for $y \in \mathrm{X}^{\mathrm{M}_{0}}$ and therefore the linear form $\mathrm{J}\left(x, \alpha_{y}^{\varrho^{\prime}}, \lambda\right)$ on $\mathrm{I}_{\mathrm{M}_{0}}^{\mathrm{G}}(\varrho)$ is given by the meromorphic continuation of the sum of integrals

$$
\begin{aligned}
& \mathrm{J}\left(\varphi: x, \alpha_{y}^{\varrho^{\prime}}, \lambda\right) \\
& \quad=\mathrm{L}(1, \eta)^{n} \eta(\operatorname{det} x) \sum_{y \in\left(\mathrm{M}_{0}^{\prime} \cap x \bullet \mathrm{G}\right) / \mathrm{M}_{0}}\left(v_{0} \varrho^{\prime}\right)(y) e^{\frac{1}{2}\langle\rho+\lambda, \mathrm{H}(y)\rangle} \int_{\mathrm{M}_{1} \backslash \mathrm{G} y} \varphi_{\lambda}\left(g \iota_{y}^{x}\right) d g .
\end{aligned}
$$

Up to the factor $\mathrm{L}(1, \eta)^{n}$, this is also the "stable intertwining period" considered in [Off07]. (The terminology comes from the analogy with the stabilization of LabesseLanglands [LL79].) The normalized open periods are closely related to Hironaka's spherical functions on X (cf. [Off07, §3.2]). Using this relation, (4.6) and the explicit formulas of [Hir99] one gets

$$
\mathcal{J}_{\varrho^{\prime}}\left(\varphi_{0}: x, \alpha^{\varrho^{\prime}}, \lambda\right)=\mathrm{L}(1, \eta)^{n} \prod_{i<j} \frac{\mathrm{L}\left(1-\left(\lambda_{i}-\lambda_{j}\right), \varrho_{j}^{\prime}\left(\varrho_{i}^{\prime}\right)^{-1} \eta\right)}{\mathrm{L}\left(1+\lambda_{i}-\lambda_{j}, \varrho_{i}^{\prime}\left(\varrho_{j}^{\prime}\right)^{-1}\right)}
$$

for $x \in \mathrm{X} \cap \mathrm{K}=e \bullet \mathrm{K}[\mathrm{Off09},(7.13)]$.

Let $\mathcal{H}=\mathcal{H}(\mathrm{G}, \mathrm{K})=\mathcal{S}(\mathrm{K} \backslash \mathrm{G} / \mathrm{K})\left(\right.$ resp. $\left.\mathcal{H}^{\prime}\right)$ be the spherical Hecke algebra of $\mathrm{G}$ (resp. $\left.\mathrm{G}^{\prime}\right)$. The homomorphism bc $: \mathcal{H} \rightarrow \mathcal{H}^{\prime}$ defined by $\widehat{\mathrm{bc}(f)}\left(\pi^{\prime}\right)=\widehat{f}\left(\mathrm{bc}\left(\pi^{\prime}\right)\right)$ is injective and identifies $\mathcal{H}^{\prime}$ as an $\mathcal{H}$-algebra which is free of rank $2^{n}$. Let $\mathcal{M}=\mathcal{S}(\mathrm{X} / \mathrm{K})$. It is naturally an $\mathcal{H}$-module via convolution. Hironaka defined in [Hir99] a spherical Fourier transform $\Phi \mapsto \widehat{\Phi}$ on $\mathcal{M}$. In terms of the local open periods it is given by

$$
\widehat{\Phi}\left(\pi^{\prime}\right)=\mathcal{J}_{\mathrm{I}_{\mathrm{M}}^{\prime}}\left(\varphi_{0}: e, \alpha^{1_{\mathrm{M}_{0}^{\prime}}}, \lambda\right)^{-1} \int_{\mathrm{X}} \Phi(x) \mathcal{J}_{\mathrm{l}_{\mathrm{M}_{0}^{\prime}}}\left(\varphi_{0}: x, \alpha^{1 \mathrm{M}_{0}^{\prime}}, \lambda\right) d x
$$

where $\varphi_{0} \in \mathrm{I}\left(1_{\mathrm{M}_{0}}\right)$ is the standard spherical section and $\pi^{\prime}=\mathrm{I}\left(1_{\mathrm{M}_{0}^{\prime}}, \lambda\right)$.

By [Hir99, Theorem 2] we have an isomorphism of $\mathcal{H}$-modules between $\mathcal{H}^{\prime}$ and $\mathcal{M}$ given by $f^{\prime} \mapsto \Phi$ if $\widehat{f^{\prime}}=\widehat{\Phi}$.

Recall that $\mathbf{W}_{e}\left(\varphi_{0}^{\prime}: \varrho^{\prime}, \lambda\right)=\mathfrak{c s}\left(\varrho^{\prime}, \lambda\right)^{-1}$ where $\varphi_{0}^{\prime} \in \mathrm{I}\left(\varrho^{\prime}\right)$ is the standard spherical section and $\mathfrak{c s}$ was defined in (1.9) [CS80]. Taking our normalization into account we recall the following result. 
Theorem 8.1.

(1) We have $\Pi_{\mathrm{unr}}^{\mathrm{M}_{0}^{\prime}} \subseteq \mathcal{B I}_{\mathrm{M}_{0}^{\prime}}$ and for any $\varrho^{\prime} \in \Pi_{\mathrm{unr}}^{\mathrm{M}_{0}^{\prime}}$ we have

$$
\alpha_{\mathrm{M}_{0}}\left(\varrho^{\prime}, \lambda\right)=\mathcal{J}_{\varrho^{\prime}}\left(\alpha^{\varrho^{\prime}}, \lambda\right)
$$

as meromorphic functions in $\lambda \in \mathfrak{a}_{\mathrm{M}_{0}, \mathbf{G}}^{*}$.

(2) Assume that $\pi^{\prime}=\mathrm{I}\left(\varrho^{\prime}, \lambda\right) \in \Pi_{\mathrm{unr}, \mathrm{bc}-\mathrm{gen}}$. Then $\pi^{\prime} \in \mathcal{B I}$ and we have

$$
\alpha^{\pi^{\prime}} \circ \mathbf{W}(\varrho, \lambda)=\mathcal{J}_{\varrho^{\prime}}\left(\alpha^{\varrho^{\prime}}, \lambda\right) .
$$

In particular, $\mathcal{J}_{\varrho^{\prime}}\left(\alpha^{\varrho^{\prime}}, \cdot\right)$ is holomorphic at $\lambda$.

(3) For any $f^{\prime} \in \mathcal{H}^{\prime}$ and $\Phi \in \mathcal{M}$ such that $\widehat{\Phi}=\widehat{f}^{\prime}$ we have $\Phi \longleftrightarrow f^{\prime}$. Moreover, for any $\varrho^{\prime} \in \Pi_{\mathrm{unr}}^{\mathrm{M}_{0}^{\prime}}$ we have the identity

$$
\tilde{\mathbf{B}}\left(\Phi: \varrho^{\prime}, \lambda\right)=\mathbf{B}\left(f^{\prime}: \varrho^{\prime}, \lambda\right)
$$

of meromorphic functions in $\lambda \in \mathfrak{a}_{\mathrm{M}, \mathbf{G}}^{*}$.

(4) Suppose that $\mu \in\left(\mathfrak{a}_{0, \mathbf{G}}^{\mathrm{M}}\right)^{*}$ and $\sigma^{\prime}=\mathrm{I}_{\mathrm{M}_{0}^{\prime}}^{\mathrm{M}^{\prime}}\left(\varrho^{\prime}, \mu\right) \in \Pi_{\mathrm{unr}, \mathrm{bc} \text {-gen }}^{\mathrm{M}^{\prime}}$ and let $\sigma=\mathrm{I}_{\mathrm{M}_{0}}^{\mathrm{M}}(\varrho, \mu)$. Then as rational functions in $q_{\mathrm{F}}^{\lambda}, \lambda \in \mathfrak{a}_{\mathrm{M}, \mathbf{c}}^{*}$ we have

$$
\mathcal{J}_{\mathrm{M}, \sigma^{\prime}}\left(\alpha^{\sigma^{\prime}}, \lambda\right) \circ \mathrm{I}_{\mathrm{M}}^{\mathrm{G}}\left(\mathbf{W}_{\mathrm{M}_{0}}^{\mathrm{M}}(\varrho, \mu)\right) \circ \Gamma_{\mathrm{M}_{0}, \mathrm{M}}^{\mathrm{G}}(\varrho, \mu)=\mathcal{J}_{\mathrm{M}_{0}, \varrho^{\prime}}\left(\alpha^{\varrho^{\prime}}, \lambda+\mu\right) .
$$

Moreover $\sigma^{\prime} \in \mathcal{B I}_{\mathrm{M}^{\prime}}$ and

$$
\alpha\left(\sigma^{\prime}, \lambda\right)=\mathcal{J}_{\mathrm{M}, \sigma^{\prime}}\left(\alpha^{\sigma^{\prime}}, \lambda\right)
$$

Proof. - By Lemma 3.7 and $(\mathbf{4 . 6})$ we may reduce the first part to the case where $\varrho^{\prime}=1$. This case is [Off09, Theorem 7.1]. The second part follows from Lemma 3.6. The third part follows from [Off09, Theorem 10.1] and the first part.

To show the last part, observe that $(\mathbf{8 . 4})$ follows from $(\mathbf{8 . 3})$ and (4.9). Let $\mathrm{A}=\mathrm{I}_{\mathrm{M}}^{\mathrm{G}}\left(\mathbf{W}_{\mathrm{M}_{0}}^{\mathrm{M}}(\varrho, \mu)\right) \circ \Gamma_{\mathrm{M}_{0}, \mathrm{M}}^{\mathrm{G}}(\varrho, \mu)$ and $\hat{\mathrm{A}}=\mathrm{I}_{\mathrm{M}}^{\mathrm{G}}\left(\mathbf{W}_{\mathrm{M}_{0}}^{\mathrm{M}}\left(\varrho^{\vee},-\mu\right)\right) \circ \Gamma_{\mathrm{M}_{0}, \mathrm{M}}^{\mathrm{G}}\left(\varrho^{\vee},-\mu\right)$. Then $(\mathrm{A}, \hat{\mathrm{A}}): \mathrm{I}(\mathfrak{W}(\varrho), \mu+\lambda) \rightarrow \mathrm{I}(\mathfrak{W}(\sigma), \lambda)$ is an equivalence of dual couples (Proposition A.2). It follows from $(\mathbf{1 . 6})$ that $\mathbf{W}_{\mathrm{M}}\left(\sigma^{\vee},-\lambda\right)=\mathbf{W}_{\mathrm{M}_{0}}\left(\varrho^{\vee},-\mu-\lambda\right) \circ \hat{\mathrm{A}}^{-1}$. By part (1) of the Theorem, (2.9) (applied to $\left(\mathrm{A}^{-1}, \hat{\mathrm{A}}^{-1}\right)$ ) and $(\mathbf{2 . 1 1})$ we have $\sigma^{\prime} \in \mathcal{B I}_{\mathrm{M}^{\prime}}$ and $\alpha\left(\sigma^{\prime}, \lambda\right)=\alpha\left(\varrho^{\prime}, \mu+\lambda\right) \circ \mathrm{A}^{-1}$. Therefore $(\mathbf{8 . 5})$ follows from $(\mathbf{8 . 2})$ and $(\mathbf{8 . 4})$.

Finally, we provide an explicit formula for the open period for general $\mathrm{M}$ and unramified data.

Lemma 8.2. - Assume that $\sigma^{\prime}=\sigma_{1}^{\prime} \otimes \cdots \otimes \sigma_{t}^{\prime} \in \Pi_{\mathrm{unr}, \mathrm{bc} \text {-gen }}^{\mathrm{M}^{\prime}}$ and let $\sigma=\mathrm{bc}\left(\sigma^{\prime}\right)$ and $\xi_{0} \in \mathrm{I}_{\mathrm{M}}^{\mathrm{G}}(\mathcal{W}(\sigma))$ be the spherical section normalized by $\left[\xi_{0}(e)\right](e)=1$. For $x \in e \bullet \mathrm{K}$ we have

$$
\mathrm{J}_{\mathrm{M}}^{\mathrm{G}}\left(\xi_{0}: x, \alpha^{\sigma^{\prime}}, \lambda\right)=\left[\prod_{i=1}^{t} \mathrm{~L}\left(1, \sigma_{i}^{\prime} \times \sigma_{i}^{\prime \vee} \cdot \eta\right)\right] \prod_{i<j} \frac{\mathrm{L}\left(\lambda_{i}-\lambda_{j}, \sigma_{i}^{\prime} \times \sigma_{j}^{\prime \vee} \cdot \eta\right)}{\mathrm{L}\left(1+\lambda_{i}-\lambda_{j}, \sigma_{i}^{\prime} \times \sigma_{j}^{\prime \vee}\right)} .
$$


Proof. — Write $\sigma^{\prime}=\mathrm{I}_{\mathrm{M}_{0}^{\prime}}^{\mathrm{M}^{\prime}}\left(\varrho^{\prime}, \mu\right)$ and note that $\xi_{0}=\mathfrak{c s}^{\mathrm{M}}(\varrho, \mu) \mathrm{I}_{\mathrm{M}}\left(\mathbf{W}_{\mathrm{M}_{0}}^{\mathrm{M}}(\varrho, \mu)\right) \circ$ $\Gamma_{\mathrm{M}_{0}, \mathrm{M}}^{\mathrm{G}}(\varrho, \mu) \varphi_{0}$ where $\varphi_{0}$ is the standard section of $\mathrm{I}_{\mathrm{M}_{0}}(\varrho)$. Therefore by $(\mathbf{8 . 4}) \mathrm{J}_{\mathrm{M}}^{\mathrm{G}}\left(\xi_{0}\right.$ : $\left.x, \alpha^{\sigma^{\prime}}, \lambda\right)$ equals

$$
\begin{aligned}
& \mathfrak{n}_{\mathrm{M}^{\prime}}^{\mathrm{G}^{\prime}}\left(\sigma^{\prime}, \lambda\right)^{-1} \mathcal{J}_{\mathrm{M}, \sigma^{\prime}}\left(\xi_{0}: \alpha^{\sigma^{\prime}}, \lambda\right) \\
& \quad=\mathfrak{n}_{\mathrm{M}^{\prime}}^{\mathrm{G}^{\prime}}\left(\sigma^{\prime}, \lambda\right)^{-1} \mathfrak{c s}^{\mathrm{M}}(\varrho, \mu) \mathcal{J}_{\mathrm{M}_{0}, \varrho^{\prime}}\left(\varphi_{0}: \alpha^{\varrho^{\prime}}, \lambda+\mu\right) .
\end{aligned}
$$

On the other hand, from the identity $(\mathbf{8 . 1}) \mathcal{J}_{\mathrm{M}_{0}, \varrho^{\prime}}\left(\varphi_{0}: \alpha^{\varrho^{\prime}}, \lambda+\mu\right)$ equals

$$
\mathrm{L}(1, \eta)^{n} \frac{\mathfrak{c s}^{\mathrm{M}}\left(\varrho, w_{0}^{\mathrm{M}} \mu\right)}{\mathfrak{c s}^{\mathrm{M}^{\prime}}\left(\varrho^{\prime}, w_{0}^{\mathrm{M}} \mu\right) \mathfrak{c s}^{\mathrm{M}^{\prime}}\left(\varrho^{\prime}, \mu\right)} \prod_{1 \leq i<j \leq t} \frac{\mathrm{L}\left(1-\left(\lambda_{i}-\lambda_{j}\right), \sigma_{i}^{\prime \vee} \times \sigma_{j}^{\prime} \cdot \eta\right)}{\mathrm{L}\left(1+\lambda_{i}-\lambda_{j}, \sigma_{i}^{\prime} \times \sigma_{j}^{\prime \vee}\right)}
$$

The lemma follows from the easily verified identities

$$
\mathrm{L}(1, \eta)^{n} \frac{\mathfrak{c s}^{\mathrm{M}}\left(\varrho, w_{0}^{\mathrm{M}} \mu\right) \mathfrak{c s}^{\mathrm{M}}(\varrho, \mu)}{\mathfrak{c s}^{\mathrm{M}^{\prime}}\left(\varrho^{\prime}, w_{0}^{\mathrm{M}} \mu\right) \mathfrak{c s}^{\mathrm{M}^{\prime}}\left(\varrho^{\prime}, \mu\right)}=\prod_{i=1}^{t} \mathrm{~L}\left(1, \sigma_{i}^{\prime} \times \sigma_{i}^{\prime \vee} \cdot \eta\right)
$$

and

$$
\begin{aligned}
& \mathfrak{n}_{\mathrm{M}^{\prime}}^{\mathrm{G}^{\prime}}\left(\sigma^{\prime}, \lambda\right)^{-1} \prod_{1 \leq i<j \leq t} \frac{\mathrm{L}\left(1-\left(\lambda_{i}-\lambda_{j}\right), \sigma_{i}^{\prime \vee} \times \sigma_{j}^{\prime} \cdot \eta\right)}{\mathrm{L}\left(1+\lambda_{i}-\lambda_{j}, \sigma_{i}^{\prime} \times \sigma_{j}^{\prime \vee}\right)} \\
& =\prod_{1 \leq i<j \leq t} \frac{\mathrm{L}\left(\lambda_{i}-\lambda_{j}, \sigma_{i}^{\prime} \times \sigma_{j}^{\prime \vee} \cdot \eta\right)}{\mathrm{L}\left(1+\lambda_{i}-\lambda_{j}, \sigma_{i}^{\prime} \times \sigma_{j}^{\prime \vee}\right)} .
\end{aligned}
$$

8.2. Closed periods and Bessel distributions. - Denote by $\mathrm{M}_{0, k}$ the standard minimal Levi subgroup of $\mathrm{G}_{k}$. We have the following identity.

Lemma 8.3. - Let $n=2 k, \sigma^{\prime}=1_{\mathrm{M}_{0, k}^{\prime}} \otimes \eta 1_{\mathrm{M}_{0, k}^{\prime}}$ and $\lambda \in\left(\mathfrak{a}_{\mathrm{M}_{0}}^{*}\right)_{\mathbf{G}}^{w_{0}}$. Let $\left.\varphi_{0} \in \mathrm{I}_{\left(\mathrm{M}_{0}\right.}\right)$ be the standard spherical section. Then for $x \in \mathrm{X} \cap \mathrm{K}$ we have

$$
\mathcal{J}_{\sigma^{\prime}}\left(\varphi_{0}: x, \alpha^{\sigma^{\prime}}, \lambda\right)=\mathrm{Z}\left(\varphi_{0}: x, \beta^{\mathrm{l}_{\mathrm{M}_{0}}}, \lambda\right)
$$

Furthermore,

$$
\tilde{\mathbf{B}}\left(\mathbf{1}_{\mathrm{X} \cap \mathrm{K}}: \sigma^{\prime}, \lambda\right)=\tilde{\mathbf{D}}\left(\mathbf{1}_{\mathrm{X} \cap \mathrm{K}}: 1_{\mathrm{M}_{0}}, \lambda\right)
$$


Proof. - First note that both sides of $(\mathbf{8 . 6})$ are independent of $x \in \mathrm{X} \cap \mathrm{K}$ since they are $\mathrm{K}$-invariant and $\mathrm{X} \cap \mathrm{K}=e \bullet \mathrm{K}$. We have $\lambda_{n+1-i}=\lambda_{i}$ for all $i$ where $\lambda=\left(\lambda_{1}, \ldots, \lambda_{n}\right)$. By $(\mathbf{8 . 1})$ for $\varrho^{\prime}=\sigma^{\prime}$ the left-hand side of $(\mathbf{8 . 6})$ is equal to

$$
\begin{aligned}
\mathrm{L}(1, \eta)^{n}\left[\prod_{1 \leq i<j \leq k} \frac{\mathrm{L}\left(1-\left(\lambda_{i}-\lambda_{j}\right), \eta\right)}{\mathrm{L}\left(1+\lambda_{i}-\lambda_{j}, 1_{\mathrm{F}^{*}}\right)} \frac{\mathrm{L}\left(1-\left(\lambda_{j}-\lambda_{i}\right), \eta\right)}{\mathrm{L}\left(1+\lambda_{j}-\lambda_{i}, 1_{\mathrm{F}^{*}}\right)}\right] \\
\quad \times\left[\prod_{1 \leq i, j \leq k} \frac{\mathrm{L}\left(1-\left(\lambda_{j}-\lambda_{i}\right), 1_{\mathrm{F}^{*}}\right)}{\mathrm{L}\left(1+\lambda_{j}-\lambda_{i}, \eta\right)}\right] .
\end{aligned}
$$

The non-diagonal terms cancel out and we remain with $\mathrm{L}(1, \eta)^{k} \mathrm{~L}\left(1,1_{\mathrm{F}^{*}}\right)^{k}=\mathrm{L}\left(1,1_{\mathrm{E}^{*}}\right)^{k}$.

On the other hand, by (5.6) the right-hand side of $(\mathbf{8 . 6})$ is equal to $\beta^{1_{\mathrm{M}_{0, k}}}\left(\left(\varphi_{0}\right)_{\lambda}(e)\right)$ since $\mathrm{G}^{w_{0}}=\mathrm{P}_{0}^{w_{0}} \mathrm{~K}^{w_{0}}$. By our normalization of the inner product on the Whittaker model (on $\left.1_{\mathrm{M}_{0, k}}\right)$ we get $\mathrm{L}\left(1,1_{\mathrm{E}^{*}}\right)^{k}$ once again. We infer $(\mathbf{8 . 6})$.

The second part follows from $(\mathbf{8 . 6})$ and the definitions of the normalized Bessel distributions using (2.10) applied to $v_{0}=\hat{v}_{0}=\varphi_{0} \in \mathrm{I}\left(1_{\mathrm{M}_{0}}\right)$.

Corollary 8.4. - Let $\mathrm{M}$ be even symmetric and $\varrho \in \Pi_{\mathrm{unr}, \mathrm{Men}}^{\mathrm{M}, w_{0}}$. Let $\lambda \in\left(\mathfrak{a}_{\mathrm{M}}^{*}\right)_{\mathbf{C}}^{w_{0}}$ be such that $\mathrm{I}(\varrho, \lambda)$ is irreducible and let $\pi^{\prime} \in \mathcal{B}(\mathrm{I}(\varrho, \lambda))$ be such that $\pi^{\prime}=\pi^{\prime} \cdot \eta$. (Recall that this $\pi^{\prime}$ is unique.) Then

$$
\mathbf{B}_{\pi^{\prime}}\left(\mathbf{1}_{\mathrm{K}^{\prime}}\right)=\tilde{\mathbf{D}}\left(\mathbf{1}_{\mathrm{X} \cap \mathrm{K}}: \varrho, \lambda\right)
$$

Proof. - There exists $\mu \in\left(\mathfrak{a}_{\mathrm{M}}^{*}\right)_{\mathbf{C}}^{w_{0}}$ such that $\varrho=\mathrm{I}_{\mathrm{M}_{0}}^{\mathrm{M}}\left(1_{\mathrm{M}_{0}}, \mu\right)$. Then $\pi^{\prime} \simeq$ $\mathrm{I}_{\mathrm{M}_{0}^{\prime}}^{\mathrm{G}^{\prime}}\left(v^{\prime}, \mu+\lambda\right)$ where $v^{\prime}=1_{\mathrm{M}_{0, k}^{\prime}} \otimes \eta 1_{\mathrm{M}_{0, k}^{\prime}}$. It follows from Corollary 5.10 that

$$
\tilde{\mathbf{D}}(\varrho, \lambda)=\tilde{\mathbf{D}}\left(1_{\mathrm{M}_{0}}, \mu+\lambda\right)
$$

and from $(\mathbf{2 . 1 4})$ that

$$
\mathbf{B}_{\pi^{\prime}}=\mathbf{B}\left(v^{\prime}, \mu+\lambda\right) .
$$

The Corollary therefore follows from Lemma 8.3.

\section{Dependence on additive character}

So far we fixed a non-trivial character $\psi^{\prime}$ of $\mathrm{F}$. In this section we examine the dependence on $\psi^{\prime}$ of the various objects and identities. To indicate the dependence on $\psi^{\prime}$ in the notation (which was mostly suppressed so far) we append it as a superscript.

For the rest of this section fix $a \in \mathrm{F}^{*}$ and let $\psi_{a}^{\prime}=\psi^{\prime}(a \cdot)$.

Firstly, for $\pi_{i} \in \mathcal{R}_{\mathrm{pi}}\left(\mathrm{G}_{n_{i}}\right), i=1,2$ we have

$$
\varepsilon\left(s, \pi_{1} \times \pi_{2} ; \psi_{a}\right)=|a|_{\mathrm{E}}^{n_{1} n_{2}\left(s-\frac{1}{2}\right)} \omega_{\pi_{1}}^{n_{2}}(a) \omega_{\pi_{2}}^{n_{1}}(a) \varepsilon\left(s, \pi_{1} \times \pi_{2} ; \psi\right),
$$




$$
\gamma\left(s, \pi_{1} \times \pi_{2} ; \psi_{a}\right)=|a|_{\mathrm{E}}^{n_{1} n_{2}\left(s-\frac{1}{2}\right)} \omega_{\pi_{1}}^{n_{2}}(a) \omega_{\pi_{2}}^{n_{1}}(a) \gamma\left(s, \pi_{1} \times \pi_{2} ; \psi\right) .
$$

Thus, for $\sigma=\sigma_{1} \otimes \cdots \otimes \sigma_{t} \in \mathcal{R}_{\mathrm{pi}}(\mathrm{M}), \lambda=\left(\lambda_{1}, \ldots, \lambda_{t}\right) \in \mathfrak{a}_{\mathrm{M}, \mathbf{C}}^{*}$ and $w \in \mathrm{W}(\mathrm{M})$ we have

$$
\mathrm{C}_{\mathrm{M}}\left(w: \sigma, \lambda ; \psi_{a}\right)=|a|_{\mathrm{E}}^{-\frac{1}{2} \sum_{i<j: w(i)>w(j)} n_{i} n_{j}}\left[\prod_{\substack{1 \leq i<j \leq t \\ w(i)>w(j)}} \omega_{\sigma_{i}\left[\lambda_{i}\right]}(a)^{n_{j}} \omega_{\sigma_{j}\left[\lambda_{j}\right]}(a)^{-n_{i}}\right]
$$

$$
\times \mathrm{G}_{\mathrm{M}}(w: \sigma, \lambda ; \psi)
$$

In particular

$$
\mathrm{C}_{\mathrm{M}}\left(w_{\mathrm{M}}: \sigma, \lambda ; \psi_{a}\right)=|a|_{\mathrm{F}}^{-\frac{1}{2} \operatorname{dim} \mathrm{U}}\left[\prod_{i<j} \omega_{\sigma_{i}\left[\lambda_{i}\right]}(a)^{n_{j}} \omega_{\sigma_{j}\left[\lambda_{j}\right]}(a)^{-n_{i}}\right] \mathrm{C}_{\mathrm{M}}\left(w_{\mathrm{M}}: \sigma, \lambda ; \psi\right)
$$

The measure on $\mathrm{F}$ satisfies $d^{\psi_{a}^{\prime}} x=|a|_{\mathrm{F}}^{\frac{1}{2}} d^{\psi^{\prime}} x$. Accordingly, for any algebraic variety $\mathbf{Y}$ over $\mathrm{F}$ with a fixed gauge form $\boldsymbol{\omega}_{\mathbf{Y}}$ and constant $\lambda_{\mathbf{Y}}$, the measure $d y$ on $\mathrm{Y}$ satisfies $d^{\psi_{a}^{\prime}} y=|a|_{\mathrm{F}}^{\frac{1}{2} \operatorname{dim} \mathbf{Y}} d^{\psi^{\prime}} y$. It follows that

$$
\begin{aligned}
\mathrm{M}^{\psi_{a}}(w, \sigma, \lambda) & =|a|_{\mathrm{F}}^{\frac{1}{2}\left(\operatorname{dim}\left(\mathrm{U}_{w \kappa} \cap w \mathrm{U} w^{-1}\right) \backslash \mathrm{U}_{w \kappa}\right)} \mathrm{M}^{\psi}(w, \sigma, \lambda) \\
& =|a|_{\mathrm{E}}^{\frac{1}{2} \sum_{i<j: w(i)>w(j)} n_{i} n_{j}} \mathrm{M}^{\psi}(w, \sigma, \lambda)
\end{aligned}
$$

and combined with $(\mathbf{9 . 1})$ we get

$$
\mathrm{N}^{\psi_{a}}(w, \sigma, \lambda)=\left[\prod_{\substack{1 \leq i<j \leq t \\ w(i)>w(j)}} \omega_{\sigma_{i}\left[\lambda_{i}\right]}(a)^{n_{j}} \omega_{\sigma_{j}\left[\lambda_{j}\right]}(a)^{-n_{i}}\right] \mathrm{N}^{\psi}(w, \sigma, \lambda) .
$$

For $\pi \in \mathcal{R}(\mathrm{G})$ and $\alpha \in \mathcal{E}_{\mathrm{G}}\left(\mathrm{X}, \pi^{*}\right)$ the notation $\Phi \odot \alpha$ implicitly depends on the choice of $\psi^{\prime}$ through the measure on $\mathrm{X}$. Specifically

$$
\Phi \odot^{\psi_{a}^{\prime}} \alpha=|a|_{\mathrm{F}}^{\frac{1}{2} n^{2}} \Phi \odot^{\psi^{\prime}} \alpha .
$$

Let $t_{a}=t_{a, n}=\operatorname{diag}\left(a^{n-1}, \ldots, a, 1\right)$ and note that $\left(\psi_{a}\right)_{0}=\psi_{0}\left(t_{a} \cdot t_{a}^{-1}\right)$. Thus, for $\pi \in \Pi_{\text {gen }}$ and $\mathrm{W} \in \mathcal{W}^{\psi}(\pi)$ we have $\mathrm{W}\left(t_{a} \cdot\right) \in \mathcal{W}^{\psi_{a}}(\pi)$. Furthermore,

$$
\left[\mathrm{W}\left(t_{a} \cdot\right), \mathrm{W}^{\vee}\left(t_{a} \cdot\right)\right]^{\psi_{a}}=|a|_{\mathrm{E}}^{\left(\begin{array}{l}
n \\
3
\end{array}\right)+\frac{1}{2}\left(\begin{array}{l}
n \\
2
\end{array}\right)}\left[\mathrm{W}, \mathrm{W}^{\vee}\right]^{\psi}, \quad \mathrm{W} \in \mathcal{W}^{\psi}(\pi), \mathrm{W}^{\vee} \in \mathcal{W}^{\psi^{-1}}\left(\pi^{\vee}\right) .
$$

The identity follows by making a change of variable $p \mapsto t_{a}^{-1} p$ in the integral $(\mathbf{A . 1})$ defining $\mathrm{I}\left(\mathrm{W}\left(t_{a} \cdot\right), \mathrm{W}^{\vee}\left(t_{a} \cdot\right), s\right)$ and noting that the Jacobian is $\delta_{\mathcal{Q}_{n}}\left(t_{a}\right)^{-1} \delta_{\mathrm{P}_{0}}\left(t_{a}\right)=|a|_{\mathrm{E}}^{\left(\begin{array}{c}n+1 \\ 3\end{array}\right)-\left(\begin{array}{c}n \\ 2\end{array}\right)}=|a|_{\mathrm{E}}^{\left(\begin{array}{c}n \\ 3\end{array}\right)}$, 
while the measure changes by the factor $|a|_{\mathrm{E}}^{\frac{1}{2}\left(\begin{array}{l}n \\ 2\end{array}\right)}$. Let $\mathrm{A}_{\pi}^{\psi, a}: \mathcal{W}^{\psi}(\pi) \rightarrow \mathcal{W}^{\psi_{a}}(\pi)$ be defined by

$$
\mathrm{A}_{\pi}^{\psi, a} \mathrm{~W}=\mathrm{W}^{a}=|a|_{\mathrm{E}}^{-\frac{1}{2}\left(\begin{array}{c}
n \\
3
\end{array}\right)-\frac{1}{4}\left(\begin{array}{c}
n \\
2
\end{array}\right)} \mathrm{W}\left(t_{a} \cdot\right) .
$$

Then by $(\mathbf{9 . 4})\left(\mathrm{A}_{\pi}^{\psi, a}, \mathrm{~A}_{\pi^{\vee}}^{\psi^{-1}, a}\right): \mathfrak{W}^{\psi}(\pi) \rightarrow \mathfrak{W}^{\psi_{a}}(\pi)$ is an equivalence of dual couples. It is also clear that

$$
\delta_{g}^{\pi, \psi_{a}} \circ \mathrm{A}_{\pi}^{\psi, a}=|a|_{\mathrm{E}}^{-\frac{1}{2}\left(\begin{array}{c}
n \\
3
\end{array}\right)-\frac{1}{4}\left(\begin{array}{c}
n \\
2
\end{array}\right)} \delta_{t_{a g}}^{\pi, \psi} .
$$

The analogous property with respect to $\mathrm{F}$ together with the relation (2.4) imply that for $\pi^{\prime} \in \Pi_{\text {gen }}^{\prime}$ we have

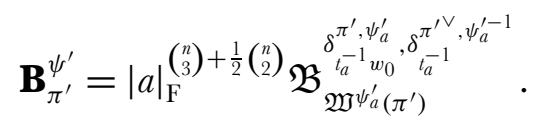

Note that

$$
\delta_{t_{a}^{-1} w_{0}}^{\pi^{\prime}, \psi_{a}^{\prime}}=\delta_{w_{0}}^{\pi^{\prime}, \psi_{a}^{\prime}} \circ \mathcal{W}^{\psi_{a}^{\prime}}\left(w_{0} t_{a}^{-1} w_{0}, \pi^{\prime}\right)=\delta_{w_{0}}^{\pi^{\prime}, \psi_{a}^{\prime}} \circ \mathcal{W}^{\psi_{a}^{\prime}}\left(z_{a}^{-(n-1)} t_{a}, \pi^{\prime}\right)
$$

where $z_{a}$ is the central element of $\mathrm{G}^{\prime}$ with $a$ in the diagonal. Hence, taking into account an extra factor of $|a|_{\mathrm{F}}^{\frac{1}{2} n^{2}}$ which comes from the change of measure on $\mathrm{G}^{\prime}$, it follows from (2.1) that

$$
\mathbf{B}_{\pi^{\prime}}^{\psi_{a}^{\prime}}\left(f_{a}^{\prime}\right)=\omega_{\pi^{\prime}}(a)^{n-1}|a|_{\mathrm{F}}^{\frac{1}{2}\left(\begin{array}{c}
n+1 \\
2
\end{array}\right)-\left(\begin{array}{l}
n \\
3
\end{array}\right)} \mathbf{B}_{\pi^{\prime}}^{\psi^{\prime}}\left(f^{\prime}\right)
$$

for every $f^{\prime} \in \mathcal{S}\left(\mathrm{G}^{\prime}\right)$ where

$$
f_{a}^{\prime}(g)=f^{\prime}\left(t_{a}^{-1} g t_{a}^{-1}\right) \text {. }
$$

For $\Phi \in \mathcal{S}(\mathrm{X})$ we have

$$
\Phi \stackrel{\psi^{\prime}}{\longleftrightarrow} f^{\prime} \quad \text { if and only if } \Phi_{a} \stackrel{\psi_{a}^{\prime}}{\longleftrightarrow} f_{a}^{\prime}
$$

where $\Phi_{a}(x)=\Phi\left(x \bullet t_{a}^{-1}\right)$. Indeed, by performing a change of variables $\left(u_{1}, u_{2}\right) \mapsto$ $\left(t_{a}^{-1} u_{1} t_{a}, t_{a}^{-1} u_{2} t_{a}\right)$ the orbital integral of $f_{a}^{\prime}$ with respect to $\psi_{a}^{\prime}$ at $t \in \mathrm{M}_{0}^{\prime}$ is $\delta_{\mathrm{P}_{0}^{\prime}}\left(t_{a}\right)^{-2}|a|_{\mathrm{F}}^{\operatorname{dim} \mathrm{U}_{0}^{\prime}}$ times the orbital integral of $f^{\prime}$ with respect to $\psi^{\prime}$ at $t_{a}^{-2} t$. Analogously, the orbital integral of $\Phi_{a}$ behaves in a compatible way.

Let $\pi^{\prime} \in \Pi_{\mathrm{bc}-\text { gen }}^{\prime}, \pi=\mathrm{bc}\left(\pi^{\prime}\right)$ and $\alpha \in \mathcal{E}_{\mathrm{G}}\left(\mathrm{X}, \mathcal{W}^{\psi}(\pi)^{*}\right)$. Define $\alpha^{a} \in$ $\mathcal{E}_{\mathrm{G}}\left(\mathrm{X}, \mathcal{W}^{\psi_{a}}(\pi)^{*}\right)$ by $\alpha_{x}^{a}\left(\mathrm{~W}^{a}\right)=\alpha_{x}(\mathrm{~W}), \mathrm{W} \in \mathcal{W}^{\psi}(\pi)$. Then as in the derivation of $(\mathbf{9 . 5})$ we have

$$
\begin{aligned}
& \tilde{\mathfrak{B}}_{\mathfrak{W}^{\psi^{*}(\pi)}}^{\alpha^{a}, \delta_{e}^{\pi^{\vee}, \psi_{a}^{-1}}}\left(\Phi_{a}\right) \stackrel{(\mathbf{2 . 9})+(\mathbf{9 . 3})}{=}|a|_{\mathrm{F}}^{\frac{1}{2} n^{2}}|a|_{\mathrm{F}}^{-\left(\begin{array}{c}
n \\
3
\end{array}\right)-\frac{1}{2}\left(\begin{array}{c}
n \\
2
\end{array}\right)} \tilde{\mathfrak{B}}_{\mathfrak{W}^{\psi}(\pi)}^{\alpha, \delta_{t^{\vee}}, \psi^{-1}} \quad\left(\Phi_{a}\right) \\
& \stackrel{(2.7)}{=}|a|_{\mathrm{F}}^{\frac{1}{2}\left(\begin{array}{c}
n+1 \\
2
\end{array}\right)-\left(\begin{array}{c}
n \\
3
\end{array}\right)} \tilde{\mathfrak{B}}_{\mathfrak{W}^{\psi}(\pi)}^{\alpha, \delta_{e} \pi^{\vee}, \psi^{-1}}(\Phi) \text {. }
\end{aligned}
$$


Hence, using (9.5) and (9.6), $\pi^{\prime} \in \mathcal{B I}_{x}^{\psi^{\prime}}$ if and only if $\pi^{\prime} \in \mathcal{B I}_{x}^{\psi_{a}^{\prime}}$ and we have

$$
\alpha_{x}^{\pi^{\prime}, \psi_{a}^{\prime}}\left(\mathrm{W}^{a}\right)=\omega_{\pi^{\prime}}(a)^{n-1} \alpha_{x}^{\pi^{\prime}, \psi^{\prime}}(\mathrm{W}), \quad \mathrm{W} \in \mathcal{W}^{\psi}(\pi) .
$$

More generally, for $\sigma \in \Pi_{\text {gen }}^{\mathrm{M}}$ let $\mathrm{A}_{\sigma}^{\psi, a}: \mathcal{W}^{\psi}(\sigma) \rightarrow \mathcal{W}^{\psi_{a}}(\sigma)$ be defined by

$$
\mathrm{A}_{\sigma}^{\psi, a} \mathrm{~W}=\mathrm{W}^{\mathrm{M}, a}=|a|_{\mathrm{E}}^{-\frac{1}{4} \sum_{i=1}^{t}\left(\begin{array}{c}
n_{i} \\
2
\end{array}\right)-\frac{1}{2} \sum_{i=1}^{t}\left(\begin{array}{c}
n_{i} \\
3
\end{array}\right)} \mathrm{W}\left(t_{a}^{\mathrm{M}} \cdot\right), \quad \mathrm{W} \in \mathcal{W}^{\psi}(\sigma)
$$

where $t_{a}^{\mathrm{M}}=\operatorname{diag}\left(t_{a, n_{1}}, \ldots, t_{a, n_{t}}\right)$. Then $\left(\mathrm{A}_{\sigma}^{\psi, a}, \mathrm{~A}_{\sigma^{\vee}}^{\psi^{-1}, a}\right): \mathfrak{W}^{\mathrm{M}, \psi}(\sigma) \rightarrow \mathfrak{W}^{\mathrm{M}, \psi_{a}}(\sigma)$ is an equivalence of dual couples.

Set further $\mathrm{A}^{\psi, a}(\sigma)=|a|_{\mathrm{F}}^{-\frac{1}{4} \operatorname{dim} \mathrm{U}_{\mathrm{M}}} \mathrm{I}_{\mathrm{M}}\left(\mathrm{A}_{\sigma}^{\psi, a}\right)$. More explicitly,

$$
\mathrm{A}^{\psi, a}(\sigma)(\varphi)(g)=\varphi^{a}(g):=|a|_{\mathrm{F}}^{-\frac{1}{4} \operatorname{dim} \mathrm{U}} \varphi(g)^{\mathrm{M}, a}=|a|_{\mathrm{E}}^{-\frac{1}{4}\left(\begin{array}{c}
n \\
2
\end{array}\right)-\frac{1}{2} \sum_{i=1}^{t}\left(\begin{array}{c}
n_{i} \\
3
\end{array}\right)} \varphi(g)\left(t_{a}^{\mathrm{M}} \cdot\right) .
$$

Then $\left(\mathrm{A}^{\psi, a}(\sigma), \mathrm{A}^{\psi^{-1}, a}\left(\sigma^{\vee}\right)\right): \mathrm{I}^{\psi}\left(\mathfrak{W}^{\psi}(\sigma), \lambda\right) \rightarrow \mathrm{I}^{\psi_{a}}\left(\mathfrak{W}^{\psi_{a}}(\sigma), \lambda\right)$ is an equivalence of dual couples for every $\lambda \in \mathfrak{a}_{\mathrm{M}, \mathbf{G}}^{*}$. (The factor $|a|_{\mathrm{F}}^{-\frac{1}{4} \operatorname{dim} \mathrm{U}}$ compensates for the change of measures on $\mathrm{P} \backslash \mathrm{G}$.)

Define the element $z_{a}^{\mathrm{M}}:=t_{a}^{\mathrm{M}} w_{\mathrm{M}}^{-1} t_{a}^{-1} w_{\mathrm{M}}$.

Lemma 9.1. - Let $\sigma \in \Pi_{\text {gen }}^{\mathrm{M}}$.

(1) We have $z_{a}^{\mathrm{M}}=\operatorname{diag}\left(a^{-l_{1}} \mathrm{I}_{n_{1}}, \ldots, a^{-l_{l}} \mathbf{I}_{n_{t}}\right)$ where $l_{j}=n_{1}+\cdots+n_{j-1}$. In particular, $z_{a}^{\mathrm{M}}$ is central in $\mathrm{M}$.

(2) For any $\sigma^{\prime} \in \mathcal{B}(\sigma)$ we have

$$
\frac{\omega_{\sigma[\lambda]}\left(z_{a}^{\mathrm{M}}\right) \omega_{\mathrm{I}\left(\sigma^{\prime}, \lambda\right)}(a)^{n-1} e^{-\left\langle\lambda, \mathrm{H}\left(t_{a}^{\mathrm{M}}\right)\right\rangle}}{\left[\prod_{i<j} \omega_{\sigma_{i}^{\prime}\left[\lambda_{i}\right]}(a)^{n_{j}} \omega_{\sigma_{j}^{\prime}\left[\lambda_{j}\right]}(a)^{-n_{i}}\right] \prod_{i=1}^{t} \omega_{\sigma_{i}^{\prime}}(a)^{n_{i}-1}}=1 .
$$

(3) We have

$$
\mathbf{W}^{\psi_{a}}(\sigma, \lambda) \circ \mathrm{A}^{\psi, a}(\sigma)=\omega_{\sigma[\lambda]}\left(z_{a}^{\mathrm{M}}\right) e^{-\left\langle\lambda, \mathrm{H}\left(t_{a}^{\mathrm{M}}\right)\right\rangle} \mathrm{A}_{\mathrm{I}(\sigma, \lambda)}^{\psi, a} \circ \mathbf{W}^{\psi}(\sigma, \lambda)
$$

whenever $\mathrm{I}(\sigma, \lambda) \in \Pi_{\text {gen }}$. In particular,

$$
\begin{aligned}
& \mathbf{W}_{e}^{\psi_{a}^{-1}}\left(\sigma^{\vee},-\lambda\right) \circ \mathrm{A}^{\psi^{-1}, a}\left(\sigma^{\vee}\right) \\
& =\omega_{\sigma[\lambda]}\left(z_{a}^{\mathrm{M}}\right)^{-1} e^{\left\langle\lambda, \mathrm{H}\left(t_{a}^{\mathrm{M}}\right)\right\rangle}|a|_{\mathrm{E}}^{-\frac{1}{4}\left(\begin{array}{c}
n \\
2
\end{array}\right)-\frac{1}{2}\left(\begin{array}{l}
n \\
3
\end{array}\right)} \mathbf{W}^{\psi^{-1}}\left(t_{a}: \sigma^{\vee},-\lambda\right) .
\end{aligned}
$$

Proof. - The first two parts are straightforward computations. For the last part, for any $\varphi \in \mathrm{I}\left(\mathcal{W}^{\psi}(\sigma)\right)$ we have 


$$
\begin{aligned}
\mathbf{W}^{\psi_{a}}\left(g: \varphi^{a}, \sigma, \lambda\right) & =\int_{\mathrm{U}_{\overleftarrow{k}}} \delta_{e}^{\sigma, \psi_{a}}\left[\left(\varphi^{a}\right)_{\lambda}\left(w_{\mathrm{M}}^{-1} u g\right)\right]\left(\psi_{a}\right)_{0}^{-1}(u) d^{\psi_{a}} u \\
& =\int_{\mathrm{U}_{\overleftarrow{\kappa}}} \delta_{e}^{\sigma, \psi_{a}}\left[\left(\varphi^{a}\right)_{\lambda}\left(w_{\mathrm{M}}^{-1} u g\right)\right] \psi_{0}^{-1}\left(t_{a} u t_{a}^{-1}\right) d^{\psi_{a}} u
\end{aligned}
$$

By a change of variable this is

$$
\delta_{\mathrm{P}_{\overleftarrow{\kappa}}}^{-1}\left(t_{a}\right) \int_{\mathrm{U}_{\overleftarrow{\kappa}}} \delta_{e}^{\sigma, \psi_{a}}\left[\left(\varphi^{a}\right)_{\lambda}\left(w_{\mathrm{M}}^{-1} t_{a}^{-1} u t_{a} g\right)\right] \psi_{0}^{-1}(u) d^{\psi_{a}} u
$$

which is equal to

$$
\begin{aligned}
& \delta_{\mathrm{P}_{\overleftarrow{\kappa}}}^{-1}\left(t_{a}\right)|a|_{\mathrm{E}}^{-\frac{1}{4}\left(\begin{array}{c}
n \\
2
\end{array}\right)-\frac{1}{2} \sum_{i=1}^{t}\left(\begin{array}{c}
n_{i} \\
3
\end{array}\right)} \\
& \quad \times \int_{\mathrm{U}_{\overleftarrow{\kappa}}} e^{\left\langle\lambda, \mathrm{H}\left(w_{\mathrm{M}}^{-1} t_{a}^{-1} u t_{a g}\right)\right\rangle} \delta_{t_{a}^{\mathrm{M}}}^{\sigma, \psi}\left[\varphi\left(w_{\mathrm{M}}^{-1} t_{a}^{-1} u t_{a} g\right)\right] \psi_{0}^{-1}(u) d^{\psi_{a}} u
\end{aligned}
$$

This is

$$
\begin{aligned}
& \delta_{\mathrm{P}_{\overleftarrow{\kappa}}}^{-1}\left(t_{a}\right)|a|_{\mathrm{E}}^{-\frac{1}{4}\left(\begin{array}{c}
n \\
2
\end{array}\right)-\frac{1}{2} \sum_{i=1}^{t}\left(\begin{array}{c}
n_{i} \\
3
\end{array}\right)} \delta_{\mathrm{P}}^{\frac{1}{2}}\left(w_{\mathrm{M}}^{-1} t_{a}^{-1} w_{\mathrm{M}}\right) e^{\left\langle\lambda, \mathrm{H}\left(w_{\mathrm{M}}^{-1} t_{a}^{-1} w_{\mathrm{M}}\right)\right\rangle} \\
& \quad \times \int_{\mathrm{U}_{\overleftarrow{\kappa}}} e^{\left\langle\lambda, \mathrm{H}\left(w_{\mathrm{M}}^{-1} u t_{a} g\right)\right\rangle} \delta_{z_{a}^{\mathrm{M}}}^{\sigma, \psi}\left[\varphi\left(w_{\mathrm{M}}^{-1} u t_{a} g\right)\right] \psi_{0}^{-1}(u) d^{\psi_{a}} u .
\end{aligned}
$$

Since $z_{a}^{\mathrm{M}}$ is central in $\mathrm{M}$ and

$$
\delta_{\mathrm{P}}\left(w_{\mathrm{M}}^{-1} t_{a}^{-1} w_{\mathrm{M}}\right)=\delta_{\mathrm{P}_{\overleftarrow{k}}}\left(t_{a}\right)
$$

we get

$$
\begin{gathered}
\delta_{\mathrm{P}_{\overleftarrow{\kappa}}}^{-\frac{1}{2}}\left(t_{a}\right)|a|_{\mathrm{E}}^{-\frac{1}{4}\left(\begin{array}{c}
n \\
2
\end{array}\right)-\frac{1}{2} \sum_{i=1}^{t}\left(\begin{array}{c}
n_{i} \\
3
\end{array}\right)} e^{\left\langle w_{\mathrm{M}} \lambda, \mathrm{H}\left(t_{a}^{-1}\right)\right\rangle} \omega_{\sigma}\left(z_{a}^{\mathrm{M}}\right) \\
\times \int_{\mathrm{U}_{\overleftarrow{\kappa}}} \delta_{e}^{\sigma, \psi}\left[\varphi_{\lambda}\left(w_{\mathrm{M}}^{-1} u t_{a} g\right)\right] \psi_{0}^{-1}(u) d^{\psi_{a}} u
\end{gathered}
$$

which is equal to

$$
\begin{aligned}
& \delta_{\mathrm{P}_{\overleftarrow{\kappa}}}^{-\frac{1}{2}}\left(t_{a}\right)|a|_{\mathrm{E}}^{-\frac{1}{2} \sum_{i=1}^{t}\left(\begin{array}{c}
n_{i} \\
3
\end{array}\right)} e^{-\left\langle\lambda, \mathrm{H}\left(t_{a}^{\mathrm{M}}\right)\right\rangle} \omega_{\sigma[\lambda]}\left(z_{a}^{\mathrm{M}}\right)|a|_{\mathrm{E}}^{\frac{1}{2}\left(\begin{array}{l}
n \\
3
\end{array}\right)}|a|_{\mathrm{F}}^{\frac{1}{2} \operatorname{dim} \mathrm{U}_{\overleftarrow{\kappa}}} \\
& \quad \times \mathrm{A}_{\mathrm{I}(\sigma, \lambda)}\left[\mathbf{W}^{\psi}(\varphi, \sigma, \lambda)\right](g) .
\end{aligned}
$$

It remains to verify (by a straightforward calculation) that

$$
|a|_{\mathrm{E}}^{\left(\begin{array}{c}
n \\
3
\end{array}\right)}|a|_{\mathrm{F}}^{\operatorname{dim}_{\overleftarrow{\kappa}} \mathrm{U}_{\overleftarrow{\kappa}}}=\delta_{\mathrm{P}_{\overleftarrow{\kappa}}}\left(t_{a}\right)|a|_{\mathrm{E}}^{\sum_{i=1}^{t}\left(\begin{array}{c}
n_{i} \\
3
\end{array}\right)} .
$$

Finally, the relation $(\mathbf{9 . 9})$ follows immediately. 
For any $\alpha \in \mathcal{E}_{\mathrm{G}}\left(\mathrm{X}, \mathrm{I}\left(\mathcal{W}^{\psi}(\sigma), \lambda\right)^{*}\right)$ let $\alpha^{a} \in \mathcal{E}_{\mathrm{G}}\left(\mathrm{X}, \mathrm{I}\left(\mathcal{W}^{\psi_{a}}(\sigma), \lambda\right)^{*}\right)$ be given by $\alpha_{x}^{a}\left(\varphi^{a}\right)=\alpha_{x}(\varphi), x \in \mathrm{X}$. Applying $(\mathbf{2 . 9}),(\mathbf{9 . 3})$ and $(\mathbf{9 . 9})$ we get

$$
\tilde{\mathfrak{B}}_{\mathrm{I}\left(\mathfrak{W}^{\psi a}(\sigma), \lambda\right)}^{\alpha^{a}, \mathbf{W}_{\sigma^{-a}}^{\alpha^{-1}\left(\sigma^{\vee},-\lambda\right)}}=|a|_{\mathrm{F}}^{\frac{1}{2}\left(\begin{array}{c}
n+1 \\
2
\end{array}\right)-\left(\begin{array}{c}
n \\
3
\end{array}\right)} \omega_{\sigma[\lambda]}\left(z_{a}^{\mathrm{M}}\right)^{-1} e^{\left\langle\lambda, \mathrm{H}\left(t_{a}^{\mathrm{M}}\right)\right\rangle} \tilde{\mathfrak{B}}_{\mathrm{I}\left(\mathfrak{W}^{\psi}(\sigma), \lambda\right)}^{\alpha, \mathbf{W}^{\psi^{-1}}\left(t_{a}, \sigma^{\vee},-\lambda\right)} .
$$

Therefore using $(\mathbf{2} .7),(\mathbf{2}$.14), $(\mathbf{9 . 5})$ and $(\mathbf{9 . 6})$ we get

Lemma 9.2. - Let $\sigma^{\prime} \in \Pi_{\mathrm{bc}-\mathrm{Men}}^{\mathrm{M}^{\prime}}$. Then $\sigma^{\prime} \in \mathcal{B I}_{\mathrm{M}^{\prime}}^{\psi^{\prime}}$ if and only if $\sigma^{\prime} \in \mathcal{B I}_{\mathrm{M}^{\prime}}^{\psi_{a}^{\prime}}$ and then

$$
\alpha_{\mathrm{M}}^{\psi_{a}^{\prime}}\left(\sigma^{\prime}, \lambda\right) \circ \mathrm{A}^{\psi, a}(\sigma)=\omega_{\sigma[\lambda]}\left(z_{a}^{\mathrm{M}}\right) \omega_{\mathrm{I}\left(\sigma^{\prime}, \lambda\right)}(a)^{n-1} e^{-\left\langle\lambda, \mathrm{H}\left(t_{a}^{\mathrm{M}}\right)\right\rangle} \alpha_{\mathrm{M}}^{\psi^{\prime}}\left(\sigma^{\prime}, \lambda\right)
$$

We turn to study the open periods. Note that for $y \in \mathrm{X}^{\mathrm{M}}$ we have $\operatorname{dim} \mathrm{G}^{y}-$ $\operatorname{dim} \mathrm{M}^{y}=n^{2}-\sum_{i=1}^{t} n_{i}^{2}=2 \operatorname{dim} \mathrm{U}^{\prime}$ and therefore by our choice of measures we have

$$
\mathrm{J}_{\mathrm{M}}^{\psi_{a}^{\prime}}(x, \alpha, \lambda)=|a|_{\mathrm{F}}^{\operatorname{dim} \mathrm{U}^{\prime}} \mathrm{J}_{\mathrm{M}}^{\psi^{\prime}}(x, \alpha, \lambda)
$$

Together with $(\mathbf{9 . 2})$ we deduce that

$$
\mathcal{J}_{\sigma^{\prime}}^{\psi_{a}^{\prime}}(\alpha, \lambda)=|a|_{\mathrm{F}}^{\frac{1}{2} \operatorname{dim} \mathrm{U}^{\prime}}\left[\prod_{i<j} \omega_{\sigma_{i}^{\prime}\left[\lambda_{i}\right]}(a)^{n_{j}} \omega_{\sigma_{j}^{\prime}\left[\lambda_{j}\right]}(a)^{-n_{i}}\right] \mathcal{J}_{\sigma^{\prime}}^{\psi^{\prime}}(\alpha, \lambda) .
$$

In particular, using $(\mathbf{9 . 7})$ we obtain

$$
\begin{aligned}
& \mathcal{J}_{\sigma^{\prime}}^{\psi_{a}^{\prime}}\left(\alpha^{\sigma^{\prime}, \psi_{a}^{\prime}}, \lambda\right) \circ \mathrm{A}^{\psi, a}(\sigma) \\
& \quad=\prod_{i=1}^{t} \omega_{\sigma_{i}^{\prime}}(a)^{n_{i}-1}\left[\prod_{i<j} \omega_{\sigma_{i}^{\prime}\left[\lambda_{i}\right]}(a)^{n_{j}} \omega_{\sigma_{j}^{\prime}\left[\lambda_{j}\right]}(a)^{-n_{i}}\right] \mathcal{J}_{\sigma^{\prime}}^{\psi^{\prime}}\left(\alpha^{\sigma^{\prime}, \psi^{\prime}}, \lambda\right) .
\end{aligned}
$$

We note that

$$
\begin{aligned}
& \tilde{\mathbf{B}}^{\psi^{\prime}}\left(\Phi: \sigma^{\prime}, \lambda\right) \\
& \stackrel{(2.7)}{=} \tilde{\mathfrak{B}}_{\mathrm{I}\left(\mathfrak{W}^{\psi}(\sigma), \lambda\right)}^{\mathcal{J}_{\sigma^{\prime}}^{\psi^{\prime}}\left(\alpha^{\sigma^{\prime}, \psi^{\prime}}, \lambda\right), \mathbf{W}^{\psi^{-1}}\left(t_{a}, \sigma^{\vee},-\lambda\right)}\left(\Phi_{a}\right) \\
& =|a|_{\mathrm{F}}^{-\frac{1}{2} n^{2}} \omega_{\sigma[\lambda]}\left(z_{a}^{\mathrm{M}}\right) e^{\left\langle-\lambda, \mathrm{H}\left(t_{a}^{\mathrm{M}}\right)\right\rangle}|a|_{\mathrm{F}}^{\frac{1}{2}\left(\begin{array}{c}
n \\
2
\end{array}\right)+\left(\begin{array}{c}
n \\
3
\end{array}\right)} \prod_{i=1}^{t} \omega_{\sigma_{i}^{\prime}}\left(a^{-1}\right)^{n_{i}-1} \\
& \times\left[\prod_{i<j} \omega_{\sigma_{i}^{\prime}\left[\lambda_{i}\right]}(a)^{-n_{j}} \omega_{\sigma_{j}^{\prime}\left[\lambda_{j}\right]}(a)^{n_{i}}\right] \tilde{\mathbf{B}}^{\psi_{a}^{\prime}}\left(\Phi_{a}, \sigma^{\prime}, \lambda\right) \\
& \stackrel{(9.8)}{=} \omega_{\pi^{\prime}}(a)^{1-n}|a|_{\mathrm{F}}^{\left(\begin{array}{c}
n \\
3
\end{array}\right)-\frac{1}{2}\left(\begin{array}{c}
n+1 \\
2
\end{array}\right)} \tilde{\mathbf{B}}^{\psi_{a}^{\prime}}\left(\Phi_{a}: \sigma^{\prime}, \lambda\right)
\end{aligned}
$$

where $\pi^{\prime}=\mathrm{I}\left(\sigma^{\prime}, \lambda\right)$ and the second equality follows from $(\mathbf{9 . 3}),(\mathbf{2 . 9}),(\mathbf{9 . 1 2})$ and $(\mathbf{9 . 9})$. 
Remark 9.3. - It follows from $(\mathbf{9 . 1 0}),(\mathbf{9 . 1 2})$ and $(\mathbf{9 . 8})$ that $(\mathbf{8 . 5})$ holds with respect to any $\psi^{\prime}$ without restriction on its conductor.

Finally, we study the closed periods. Recall the map $\mathfrak{y}_{\pi}: \mathcal{W}(\pi)^{\tilde{\theta}} \rightarrow \mathcal{W}^{\psi^{-1}}\left(\pi^{\vee}\right)$ defined in Appendix A.4. We have

$$
\omega_{\pi}(a)^{n-1} \mathrm{~A}_{\pi^{\vee}}^{\psi^{-1}, a} \circ \mathfrak{y}^{\pi, \psi}=\mathfrak{y}^{\pi, \psi_{a}} \circ \mathrm{A}_{\pi}^{\psi, a} .
$$

Suppose that $n=2 k, \mathrm{Q}=\mathrm{P}_{(k, k)}, \mathrm{L}=\mathrm{M}_{(k, k)}$ and $\mathrm{V}=\mathrm{U}_{(k, k)}$. Let $\varrho \in \Pi_{\text {gen }}^{\mathrm{L}}$ and $\beta \in$ $\mathcal{E}_{\mathrm{L}}\left(\mathrm{X}_{\mathrm{L}}, \varrho^{*}\right)$. Since $\operatorname{dim} Q^{w_{0}} \backslash \mathrm{G}^{w_{0}}=\operatorname{dim} \mathrm{Q}^{\prime} \backslash \mathrm{G}^{\prime}=\operatorname{dim} \mathrm{V}^{\prime}=k^{2}$ it follows from $(\mathbf{5 . 6})$ that

$$
\mathrm{Z}^{\psi_{a}^{\prime}}(\beta, 0)=|a|_{\mathrm{F}}^{\frac{1}{2} k^{2}} \mathrm{Z}^{\psi^{\prime}}(\beta, 0)
$$

for any $a \in \mathrm{F}^{*}$. Now assume that $\varrho=\sigma \otimes \sigma^{\tau}$ with $\sigma \in \Pi_{\text {gen }}^{\mathrm{G}_{k}}$ and let $\varrho^{\prime}=\operatorname{ai}(\sigma)$. The definition of $\beta^{\varrho}$ together with $(\mathbf{9 . 1 3})$ and $(\mathbf{9 . 4})$ imply that

$$
\beta^{\varrho, \psi_{a}} \circ \mathrm{A}_{\varrho}^{\psi, a}=\omega_{\sigma}(a)^{k-1} \beta^{\varrho, \psi} .
$$

From this relation and $(\mathbf{9 . 1 4})$ we deduce that

$$
\mathrm{Z}^{\psi_{a}^{\prime}}\left(\beta^{\varrho, \psi_{a}}, 0\right) \circ \mathrm{A}^{\psi, a}(\varrho)=\omega_{\sigma}(a)^{k-1} \mathrm{Z}\left(\beta^{\varrho, \psi}, 0\right)
$$

Together with $(\mathbf{9 . 3})$ and $(\mathbf{9 . 9})$ we can apply $(\mathbf{2 . 9})$ with the equivalence of dual couples

$$
\left(\mathrm{A}^{\psi, a}(\varrho), \mathrm{A}^{\psi^{-1}, a}\left(\varrho^{\vee}\right)\right)
$$

to infer that $\tilde{\mathbf{D}}^{\psi_{a}^{\prime}}\left(\Phi_{a}: \varrho, 0\right)$ is equal to

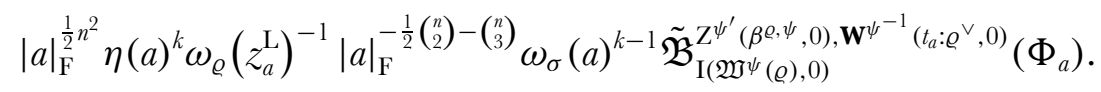

Here we also used the fact that $\boldsymbol{\lambda}_{\psi_{a}^{\prime}}=\eta(a) \boldsymbol{\lambda}_{\psi^{\prime}}$.

We have

$$
\omega_{\varrho}\left(z_{a}^{\mathrm{L}}\right)=\omega_{\sigma}(a)^{-k}
$$

On the other hand,

$$
\left.\omega_{\sigma}\right|_{\mathrm{F}^{*}}=\eta^{k} \omega_{\varrho^{\prime}} .
$$

Furthermore, clearly

$$
\mathbf{W}^{\psi^{-1}}\left(t_{a}: \varrho^{\vee}, 0\right) \circ \mathrm{I}\left(t_{a}^{-1}, \mathcal{W}\left(\varrho^{\vee}\right), 0\right)=\mathbf{W}_{e}^{\psi^{-1}}\left(\varrho^{\vee}, 0\right) .
$$

Using (2.7) with $g=t_{a}^{-1}$ we obtain

$$
\tilde{\mathbf{D}}^{\psi_{a}^{\prime}}\left(\Phi_{a}: \varrho, 0\right)=\omega_{\varrho^{\prime}}(a)^{n-1}|a|_{\mathrm{F}}^{\frac{1}{2}\left(\begin{array}{c}
n+1 \\
2
\end{array}\right)-\left(\begin{array}{c}
n \\
3
\end{array}\right)} \tilde{\mathbf{D}}^{\psi^{\prime}}(\Phi: \varrho, 0) .
$$


Now let $\kappa=\left(n_{1}, \ldots, n_{s}\right)$ be a composition of $k$ and let $n=2 k, \mathrm{Q}=\mathrm{P}_{(\kappa, \overleftarrow{\kappa})}, \mathrm{L}=$ $\mathrm{M}_{(\kappa, \overleftarrow{\kappa})}$ and $\mathrm{V}=\mathrm{U}_{(\kappa, \overleftarrow{\kappa})}$. Let $\varrho \in \Pi_{\text {gen }}^{\mathrm{L}}$ and $\beta \in \mathcal{E}_{\mathrm{L}}\left(\mathrm{X}_{\mathrm{L}}, \varrho^{*}\right)$ and $\lambda=(\mu, \overleftarrow{\mu}) \in\left(\mathfrak{a}_{\mathrm{L}}^{*}\right)_{\mathbf{G}}^{w_{0}}$ Assume that $\varrho=\sigma \otimes \overleftarrow{\sigma}^{\tau}$ with $\sigma=\sigma_{1} \otimes \cdots \otimes \sigma_{s} \in \Pi_{\text {gen }}^{\mathrm{M}_{\kappa}}$ and let $\varrho^{\prime}=$ ai $(\sigma)$. Let also $\mu^{\prime} \in \mathfrak{a}_{\mathrm{M}_{2 \kappa}^{\prime}}^{*}$ be as in Section 5.2. Applying Proposition 5.9 and $(\mathbf{9 . 1 5})$ we obtain

$$
\tilde{\mathbf{D}}^{\psi_{a}^{\prime}}\left(\Phi_{a}: \varrho, \lambda\right)=\omega_{\mathrm{I}\left(\varrho^{\prime}, \mu^{\prime}\right)}(a)^{n-1}|a|_{\mathrm{F}}^{\frac{1}{2}\left(\begin{array}{c}
n+1 \\
2
\end{array}\right)-\left(\begin{array}{l}
n \\
3
\end{array}\right)} \tilde{\mathbf{D}}^{\psi^{\prime}}(\Phi: \varrho, \lambda)
$$

\section{Global theory}

\section{Factorization of unitary periods-cuspidal representations}

We now turn to the global case and prove our main global results on factorization of periods of cusp forms and Eisenstein series. In this section and the next $\mathrm{E} / \mathrm{F}$ will be a quadratic extension of number fields.

10.1. Notation. - Let $\mathbf{A}=\mathbf{A}_{\mathrm{F}}$ denote the ring of adeles of $\mathrm{F}$ and let $\mathbf{A}^{*}$ be the group of ideles. For every place $v$ of $\mathrm{F}$ let $\mathrm{F}_{v}$ be the completion of $\mathrm{F}$ with respect to $v$ and set $\mathrm{E}_{v}=\mathrm{F}_{v} \bigotimes_{\mathrm{F}} \mathrm{E}$. For a finite place $v$ of $\mathrm{F}$ we will abbreviate by setting $\mathcal{O}_{v}=\mathcal{O}_{\mathrm{F}_{v}}$. Let $\tau$, Tr and Nm be as in the local case and let $\eta=\bigotimes_{v} \eta_{v}$ be the quadratic character attached to $\mathrm{E} / \mathrm{F}$ by class field theory, i.e., the unique non-trivial character of $\mathrm{F}^{*} \operatorname{Nm}\left(\mathbf{A}_{\mathrm{E}}^{*}\right) \backslash \mathbf{A}^{*}$. Here and elsewhere the product is taken over all places $v$ of $\mathrm{F}$. We fix a non-trivial character $\psi^{\prime}=\bigotimes_{v} \psi_{v}^{\prime}$ of $\mathbf{A}$ trivial on $\mathrm{F}$ and let $\psi=\psi^{\prime} \circ \mathrm{Tr}$. As usual, we suppress $\psi^{\prime}$ from the notation if it is clear from the context.

For an F-variety $\mathbf{Y}$ set $\mathrm{Y}_{v}=\mathbf{Y}\left(\mathrm{F}_{v}\right)$ for every place $v$ of $\mathrm{F}, \mathrm{Y}=\mathbf{Y}(\mathrm{F})$ and $\mathrm{Y}_{\mathbf{A}}=\mathbf{Y}(\mathbf{A})$. Let $\mathbf{G}^{\prime}, \mathbf{G}, \mathbf{X}, \mathbf{Z}, \mathbf{P}_{0}=\mathbf{M}_{0} \mathbf{U}_{0}$ and $\mathbf{P}_{\boldsymbol{\kappa}}=\mathbf{M}_{\kappa} \mathbf{U}_{\boldsymbol{\kappa}}$ (for a composition $\kappa$ of $n$ ) be defined as in the local case. For every $x \in \mathrm{X}_{\mathbf{A}}$ let $\mathrm{G}_{\mathbf{A}}^{x}$ be the stabilizer of $x$ in $\mathrm{G}_{\mathbf{A}}$. It is the restricted direct product of $\mathrm{G}_{v}^{x_{v}}$.

We shall fix some further notation and conventions pertaining to the group $\mathrm{G}$; the corresponding notation for $\mathrm{G}^{\prime}$ will be appended by a prime. Let $\mathbf{K}=\prod_{v} \mathrm{~K}_{v}$ be the standard maximal compact subgroup of $\mathrm{G}_{\mathbf{A}}$ where $\mathrm{K}_{v}$ is the standard maximal compact subgroup of $\mathrm{G}_{v}$ (see Section 1.1).

For a subgroup $\mathbf{Q}$ of $\mathbf{G}$ denote by $\mathrm{X}^{*}(\mathbf{Q})$ the lattice of F-rational characters of $\mathbf{Q}$ and let $\delta_{\mathbf{Q}}$ be the modulus function of $Q_{\mathbf{A}}$. Let $\mathfrak{a}_{\mathrm{Q}}^{*}$ be the real vector space $\mathrm{X}^{*}(\mathbf{Q}) \bigotimes_{\mathbf{Z}} \mathbf{R}$ and let $\mathfrak{a}_{\mathbf{Q}}$ be the dual space.

The function $\mathrm{H}: \mathrm{G}_{\mathbf{A}} \rightarrow \mathfrak{a}_{0}$ is defined by

$$
e^{\langle\chi, \mathrm{H}(g)\rangle}=\prod_{v}\left|\chi_{v}\left(m_{v}\right)\right|_{v}, \quad \chi \in \mathrm{X}^{*}\left(\mathbf{M}_{\mathbf{0}}\right)
$$

via the Iwasawa decomposition $g=u m k, u \in \mathbf{U}_{0, \mathbf{A}}, m=\left(m_{v}\right)_{v} \in \mathbf{M}_{0, \mathbf{A}}, k \in \mathbf{K}$ where $\chi_{v}$ is the extension of $\chi$ to $\mathrm{M}_{0}\left(\mathrm{~F}_{v}\right)$ for any place $v$ of $\mathrm{F}$. Note that with our conventions 
$\mathrm{H}(g)=2 \mathrm{H}^{\prime}(g)$ for all $g \in \mathrm{G}_{\mathbf{A}}^{\prime}$. Let $\mathrm{E}_{\infty}=\prod_{v \mid \infty} \mathrm{E}_{v}=\mathrm{E} \otimes_{\mathbf{Q}} \mathbf{R}$ and embed $\mathbf{R}_{+} \hookrightarrow \mathrm{E}_{\infty}^{*}$ via $x \mapsto 1 \otimes x$. Let $\mathrm{A}_{\mathrm{G}}$ be the subgroup of $\mathbf{Z}_{\mathbf{A}}$ consisting of scalar matrices which are the identity at the finite places and the scalar $x$ at the infinite part for some $x \in \mathbf{R}_{+}$. Note that $\mathrm{H}$ restricts to an isomorphism between $\mathrm{A}_{\mathrm{G}}$ and $\mathfrak{a}_{\mathrm{G}}$.

For an affine variety $\mathbf{Y}$ over $\mathrm{F}$ we define $\mathcal{S}\left(\mathrm{Y}_{\mathbf{A}}\right)=\bigotimes^{\prime} \mathcal{S}\left(\mathrm{Y}_{v}\right)$ where the restricted tensor product is with respect to the characteristic function of $\mathbf{Y}\left(\mathcal{O}_{v}\right)$ (defined for almost all $v)$.

10.2. Measures. - For $\mathbf{Y}=\mathbf{G}, \mathbf{G}^{\prime}, \mathbf{M}, \mathbf{M}^{\prime}, \mathbf{U}, \mathbf{U}^{\prime}$ or $\mathbf{X}$ we take the Tamagawa measure on $\mathrm{Y}_{\mathbf{A}}$ with respect to the convergence factors chosen in Section 1.2. This is the product of the local measures defined there with respect to $\psi_{v}^{\prime}$. Discrete groups are always endowed with the counting measure.

In particular, $\mathbf{A}_{\mathrm{F}}$ is endowed with the self-dual Haar measure with respect to $\psi^{\prime}$ and $\operatorname{vol}\left(\mathrm{F} \backslash \mathbf{A}_{\mathrm{F}}\right)=1$. The product measure on $\mathrm{X}_{\mathbf{A}}$ is compatible with the isomorphism

$$
\coprod_{\xi \in \mathrm{X}_{\mathbf{A}} / \mathrm{G}_{\mathbf{A}}} \mathrm{G}_{\mathbf{A}}^{\xi} \backslash \mathrm{G}_{\mathbf{A}} \simeq \mathrm{X}_{\mathbf{A}}
$$

where the measure on $\mathrm{G}_{\mathbf{A}}^{\xi}$ is the product measure.

10.3. Global Bessel distributions. - For our purposes, in the global case we will consider admissible representations of $\mathrm{G}_{\mathbf{A}}$ of the form $\pi=\bigotimes \pi_{v}$ (restricted tensor product with respect to $\left.e_{v}\right)$ where $\pi_{v} \in \mathcal{R}\left(\mathrm{G}_{v}\right)$ and $\pi_{v}$ is an unramified principal series with a choice of a non-zero unramified vector $e_{v}$ for almost all $v$. We can speak about dual couples $(\pi, \hat{\pi},(\cdot, \cdot))$ and define Bessel distributions, ordinary and relative, in the global setting in a way similar to the local case. Thus, a dual couple $\mathcal{D}$ gives rise to an equivalence $\Lambda_{\mathcal{D}}: \pi^{\vee}:=\bigotimes_{v} \pi_{v}{ }^{\vee} \rightarrow \hat{\pi}$, and for any $\ell \in \pi^{*}$ and $\hat{\ell} \in \hat{\pi}^{*}$ we set $\mathfrak{B}_{\mathcal{D}}^{\ell, \hat{\ell}}(f)=\hat{\ell}\left[\Lambda_{\mathcal{D}}(\ell \circ \pi(f))\right], f \in \mathcal{S}\left(\mathrm{G}_{\mathbf{A}}\right)$. Similarly, if $\alpha \in \mathcal{E}_{\mathrm{G}_{\mathbf{A}}}\left(\mathrm{X}_{\mathbf{A}}, \pi^{*}\right)$ (i.e., if $\alpha_{x \bullet g}=\alpha_{x} \circ \pi(g)$ for all $\left.g \in \mathrm{G}_{\mathbf{A}}, x \in \mathrm{X}_{\mathbf{A}}\right)$ we set $\tilde{\mathfrak{B}}_{\mathcal{D}}^{\alpha, \hat{\ell}}(\Phi)=\hat{\ell}\left[\Lambda_{\mathcal{D}}(\Phi \odot \alpha)\right], \Phi \in \mathcal{S}\left(\mathrm{X}_{\mathbf{A}}\right)$ where $\Phi \odot \alpha=\int_{\mathrm{X}_{\mathbf{A}}} \Phi(x) \alpha_{x} d x \in \pi^{\vee}$.

Suppose that $\mathcal{D}=\bigotimes_{v} \mathcal{D}_{v}$ where $\mathcal{D}_{v}=\left(\pi_{v}, \hat{\pi}_{v},(\cdot, \cdot)_{v}\right)$ and $\left(e_{v}, \hat{e}_{v}\right)_{v}=1$ for almost all $v$. Let $\ell=\bigotimes_{v} \ell_{v}, \ell_{v} \in \pi_{v}^{*}$ and $\hat{\ell}=\bigotimes_{v} \hat{\ell}_{v}, \hat{\ell}_{v} \in \hat{\pi}_{v}^{*}$ be factorizable functionals with $\ell_{v}\left(e_{v}\right)=\hat{\ell}_{v}\left(\hat{e}_{v}\right)=1$ for almost all $v$. Then

$$
\mathfrak{B}_{\mathcal{D}}^{\ell, \hat{\ell}}(f)=\prod_{v} \mathfrak{B}_{\mathcal{D}_{v}}^{\ell_{v}}, \hat{\ell}_{v}\left(f_{v}\right)
$$

for $f=\bigotimes f_{v}$.

We write $\mathcal{C}$ for the set of cuspidal automorphic representations of $\mathrm{G}_{\mathbf{A}}$ whose central character is trivial on $\mathrm{A}_{\mathrm{G}}$. Implicitly, any $\pi \in \mathcal{C}$ is realized on a space of (smooth) cusp forms of $\mathrm{G} \backslash \mathrm{G}_{\mathbf{A}}$. 
Given $\pi \in \mathcal{C}$, let $\mathfrak{A}_{\pi}$ be the dual couple $\left(\pi, \hat{\pi},(\cdot, \cdot)_{\mathrm{A}_{\mathrm{G}} \mathrm{G} \backslash \mathrm{G}_{\mathbf{A}}}\right)$ where $\hat{\pi} \in \mathcal{C}$ is realized on the space $\left\{\phi^{\tilde{\theta}}: \phi \in \pi\right\}$ with $\phi^{\tilde{\theta}}(g)=\phi\left(g^{\tilde{\theta}}\right)$ (where we recall that $\left.g^{\tilde{\theta}}=w_{0}{ }^{t} g^{-1} w_{0}\right)$ and

$$
(\phi, \hat{\phi})_{\mathrm{A}_{\mathrm{G}} \mathrm{G} \backslash \mathrm{G}_{\mathbf{A}}}=\int_{\mathrm{A}_{\mathrm{G}} \mathrm{G} \backslash \mathrm{G}_{\mathbf{A}}} \phi(g) \hat{\phi}(g) d g, \quad \phi \in \pi, \hat{\phi} \in \hat{\pi} .
$$

We denote by $\mathbf{W}^{\pi}(\phi)$ the $\psi_{0}$-th Fourier coefficient of a cusp form $\phi$ on $\mathrm{G}_{\mathbf{A}}$, i.e.,

$$
\mathbf{W}^{\pi}(g: \phi)=\int_{\mathrm{U}_{0} \backslash \mathrm{U}_{0, \mathbf{A}}} \phi(u g) \psi_{0}^{-1}(u) d u
$$

where $\psi_{0}$ is defined as in the local case. Also, let $\mathbf{W}_{e}^{\pi}(\phi)=\mathbf{W}^{\pi}(e: \phi)$ be the associated Whittaker functional. As in the local case, by abuse of notation $\mathbf{W}^{\hat{\pi}}$ and $\mathbf{W}_{e}^{\hat{\pi}}$ will be defined with respect to $\psi^{-1}$. We will use similar notation for $\mathrm{G}^{\prime}$.

Given $\pi^{\prime} \in \mathcal{C}^{\prime}$ define the global standard Bessel distribution

$$
\mathbf{B}_{\pi^{\prime}}=\mathfrak{B}_{\mathfrak{A}_{\pi^{\prime}}}^{\mathbf{W}^{\pi^{\prime}}\left(w_{0}\right), \mathbf{W}_{e}^{\hat{\pi}^{\prime}}} .
$$

We will factorize it according to the factorization of the inner product in the following manner (cf. [Jac01], [LO07, §2.2 $\left.]^{9}\right)$. Suppose that $\mathrm{S}$ is a finite set of places, containing the Archimedean ones, such that $\pi_{v}^{\prime}$ is unramified and $\psi_{v}^{\prime}$ has conductor $\mathcal{O}_{v}$ for all $v \notin \mathrm{S}$. For a right $\mathrm{K}^{\prime \mathrm{S}}=\prod_{v \notin \mathrm{S}} \mathrm{K}_{v}^{\prime}$-invariant cusp form $\phi^{\prime}$ in the space of $\pi^{\prime}$ which is a factorizable element of $\pi^{\prime} \simeq \bigotimes_{v} \pi_{v}^{\prime}$ (resp. $\left.\hat{\phi}^{\prime} \in\left(\hat{\pi}^{\prime}\right)^{\mathrm{K}^{\prime S}}\right)$ write $\mathbf{W}^{\pi^{\prime}}\left(g: \phi^{\prime}\right)=\prod_{v} \mathbf{W}_{v}^{\prime}(g)$ (resp. $\mathbf{W}^{\hat{\pi}^{\prime}}(g:$ $\left.\left.\hat{\phi}^{\prime}\right)=\prod_{v} \hat{\mathrm{W}}_{v}^{\prime}(g)\right)$ where $\mathrm{W}_{v}^{\prime} \in \mathcal{W}^{\psi_{v}^{\prime}}\left(\pi_{v}^{\prime}\right)\left(\operatorname{resp} . \hat{\mathrm{W}}_{v}^{\prime} \in \mathcal{W}^{\psi_{v}^{\prime-1}}\left(\hat{\pi}_{v}^{\prime}\right)=\mathcal{W}^{\psi_{v}^{\prime-1}}\left(\pi_{v}^{\prime \vee}\right)\right)$ and for all $v \notin \mathrm{S}$ the elements $\mathrm{W}_{v}^{\prime}, \hat{\mathrm{W}}_{v}^{\prime}$ are spherical and normalized by $\mathrm{W}_{v}^{\prime}(e)=\hat{\mathrm{W}}_{v}^{\prime}(e)=1$. We have

$(\mathbf{1 0 . 1})$

$$
\begin{aligned}
\left(\phi^{\prime}, \hat{\phi}^{\prime}\right)_{\mathrm{A}_{\mathrm{G}} \mathrm{G}^{\prime} \backslash \mathrm{G}_{\mathrm{A}}^{\prime}} & =\operatorname{Res}_{s=1} \mathrm{~L}^{\mathrm{S}}\left(s, \pi^{\prime} \times \pi^{\prime \vee}\right) \prod_{v \in \mathrm{S}}\left[\mathrm{W}_{v}^{\prime}, \hat{\mathrm{W}}_{v}^{\prime}\right]_{\pi_{v}^{\prime}} \\
& =\mathfrak{r} \mathfrak{l}^{\prime \pi^{\prime}} \prod_{v}\left(\mathfrak{r l}_{v}^{\prime \pi_{v}^{\prime}}\right)^{-1}\left[\mathrm{~W}_{v}^{\prime}, \hat{\mathrm{W}}_{v}^{\prime}\right]_{\pi_{v}^{\prime}}
\end{aligned}
$$

where $\mathfrak{r l}^{\mathfrak{l}^{\prime}}=\operatorname{Res}_{s=1} \mathrm{~L}\left(s, \pi^{\prime} \times \pi^{\prime \vee}\right)$ and $\mathfrak{r l}_{v}^{\prime \pi_{v}^{\prime}}=\mathrm{L}\left(1, \pi_{v}^{\prime} \times \pi_{v}^{\prime \vee}\right)$.

Let $\mathfrak{W}\left(\pi^{\prime}\right)$ be the dual couple $\left(\bigotimes_{v} \mathcal{W}\left(\pi_{v}^{\prime}\right), \bigotimes_{v} \mathcal{W}\left(\hat{\pi}_{v}^{\prime}\right), \mathfrak{r} \mathfrak{l}^{\prime \pi^{\prime}} \cdot \bigotimes_{v}\left(\left(\mathfrak{r} l_{v}^{\pi_{v}^{\prime}}\right)^{-1}[\cdot, \cdot]_{\pi_{v}^{\prime}}\right)\right)$. Then by the factorization $(\mathbf{1 0 . 1})\left(\mathbf{W}^{\pi^{\prime}}, \mathbf{W}^{\hat{\pi}^{\prime}}\right): \mathfrak{A}_{\pi^{\prime}} \rightarrow \mathfrak{W}\left(\pi^{\prime}\right)$ is an equivalence. Let $\delta_{g}^{\pi^{\prime}}$ be the evaluation map at $g$ on $\bigotimes_{v} \mathcal{W}\left(\pi_{v}^{\prime}\right)$. Applying the global analogue of $(\mathbf{2 . 4})$ we get $\mathbf{B}_{\pi^{\prime}}=\mathfrak{B}_{\mathfrak{W}\left(\pi^{\prime}\right)}^{\delta_{w_{0}^{\prime}}^{\pi_{0}},{\hat{\pi^{\prime}}}^{\prime}}$. Thus, for $f^{\prime}=\bigotimes_{v} f_{v}^{\prime} \in \mathcal{S}\left(\mathrm{G}_{\mathbf{A}}^{\prime}\right)$ we have

$$
\mathbf{B}_{\pi^{\prime}}\left(f^{\prime}\right)=\frac{1}{\mathfrak{r} \mathfrak{l}^{\prime \pi^{\prime}}} \prod_{v} \mathfrak{\mathfrak { r l } ^ { \prime } \boldsymbol { \pi } _ { v } ^ { \prime }} \mathbf{B}_{\pi_{v}^{\prime}}\left(f_{v}^{\prime}\right) .
$$

\footnotetext{
${ }^{9}$ Note the different convention of measures in [loc. cit.].
} 
We can also write this as

$$
\mathbf{B}_{\pi^{\prime}}\left(f^{\prime}\right)=\frac{\mathbf{B}_{\pi_{\mathrm{S}}^{\prime}}\left(f_{\mathrm{S}}^{\prime}\right)}{\operatorname{Res}_{s=1} \mathrm{~L}^{\mathrm{S}}\left(s, \pi^{\prime} \times \pi^{\prime \vee}\right)}
$$

whenever $f^{\prime}=f_{\mathrm{S}}^{\prime} \otimes \mathbf{1}_{\left(\mathrm{K}^{\prime}\right)} \mathrm{s}$ and $f_{\mathrm{S}}^{\prime}=\bigotimes_{v \in \mathrm{S}} f_{v}^{\prime} \in \mathcal{S}\left(\mathrm{G}_{\mathrm{S}}^{\prime}\right)$. Here $\mathbf{B}_{\pi_{\mathrm{S}}^{\prime}}\left(f_{\mathrm{S}}^{\prime}\right)=\prod_{v \in \mathrm{S}} \mathbf{B}_{\pi_{v}^{\prime}}\left(f_{v}^{\prime}\right)$. Recall that by $(\mathbf{2 . 1 7})$ this factorization is consistent with enlarging $\mathrm{S}$.

10.4. An extension of Jacquet's factorization Theorem. - For $\pi \in \mathcal{C}$ and an automorphism $J$ of $\mathbf{G}$ we denote by $\pi^{\jmath} \in \mathcal{C}$ the representation realized on the space of functions $\phi^{J}$ where $\phi$ is in the space of $\pi$ and $\phi^{J}\left(g^{J}\right)=\phi(g)$. In particular $\pi^{\tau}$ is the Galois twist of $\pi$.

Denote by $\mathcal{C}^{\tau}$ the subset of $\mathcal{C}$ consisting of the $\tau$-invariant representations. We have a functorial transfer bc from $\mathcal{C}^{\prime}$ to automorphic representations of $\mathrm{G}_{\mathbf{A}}$ [AC89]. If $\pi^{\prime} \in \mathcal{C}^{\prime}$ and $\pi^{\prime} \neq \pi^{\prime} \cdot \eta$ then $b c\left(\pi^{\prime}\right) \in \mathcal{C}^{\tau}$ and conversely, every $\pi \in \mathcal{C}^{\tau}$ is the image under bc of $\pi^{\prime} \in \mathcal{C}^{\prime}$ which is unique up to twist by $\eta$ and we have $\pi^{\prime} \neq \pi^{\prime} \cdot \eta$.

For any automorphic form $\phi$ and $x \in \mathrm{X}$ denote by $\mathcal{P}_{x}(\phi)$ the unitary period integral

$$
\mathcal{P}_{x}(\phi)=\int_{\mathrm{G}^{x} \backslash \mathrm{G}_{\mathbf{A}}^{x}} \phi(g) d g
$$

whenever absolutely convergent. We say that $\pi$ is (globally) $\mathrm{G}^{x}$-distinguished if $\mathcal{P}_{x}$ is not identically zero on the space of $\pi$.

Our goal is to extend Jacquet's Factorization Theorem [Jac01, Theorem 1] and provide a criterion for a cuspidal representation to be $\mathrm{G}^{x}$-distinguished.

Definition 10.1. - Let $\pi=\bigotimes_{v} \pi_{v} \in \mathcal{C}$. We say that unitary periods factorize compatibly on $\pi$ if at every place $v$ of $\mathrm{F}$ there exists $\alpha^{v} \in \mathcal{E}_{\mathrm{G}_{v}}\left(\mathrm{X}_{v}, \pi_{v}^{*}\right)$ such that for every $x \in \mathrm{X}$ and every pure tensor $\phi=\bigotimes_{v} \phi_{v}$ in the space of $\pi$ we have

$$
\mathcal{P}_{x}(\phi)=\prod_{v} \alpha_{x}^{v}\left(\phi_{v}\right)
$$

where almost all the factors are 1.

We recall the elements $\alpha^{\pi_{v}^{\prime}} \in \mathcal{E}_{\mathrm{G}_{v}}\left(\mathrm{X}_{v}, \pi_{v}^{*}\right)$ defined for any place $v$ in Section 3.3.

Theorem 10.2. - Let $\pi=\mathrm{bc}\left(\pi^{\prime}\right) \in \mathcal{C}^{\tau}$. Then unitary periods factorize compatibly on $\pi$. More precisely for any $x \in \mathrm{X}$ we have

$$
\begin{aligned}
\mathcal{P}_{x}(\phi) & =2 \mathrm{~L}\left(1, \pi^{\prime} \times \pi^{\prime \vee} \cdot \eta\right) \prod_{v}\left(\mathrm{~L}\left(1, \pi_{v}^{\prime} \times \pi_{v}^{\prime \vee} \cdot \eta_{v}\right)^{-1} \alpha_{x}^{\pi_{v}^{\prime}}\left(\mathrm{W}_{v}\right)\right) \\
& =2 \mathrm{~L}^{\mathrm{S}}\left(1, \pi^{\prime} \times \pi^{\prime \vee} \cdot \eta\right) \prod_{v \in \mathrm{S}} \alpha_{x}^{\pi_{v}^{\prime}}\left(\mathrm{W}_{v}\right)
\end{aligned}
$$


where $\phi$ is a cusp form in the space of $\pi$ which is a pure tensor and $\mathbf{W}^{\pi}(\phi)=\prod_{v} \mathrm{~W}_{v}$. Here $\mathrm{S}$ is a finite set of places, containing the Archimedean and the even places, such that for all $v \notin \mathrm{S}, v$ is either split or unramified in $\mathrm{E}, \psi_{v}^{\prime}$ has conductor $\mathcal{O}_{v}, \pi_{v}^{\prime}$ is unramified, $x_{v} \in \mathrm{K}_{v}$ and $\mathrm{W}_{v}$ is spherical and normalized by $\mathrm{W}_{v}(e)=1$. If moreover $\pi$ is $\mathrm{G}^{x}$-distinguished then $\pi_{v}^{\prime} \in \mathcal{B I}_{x}$ for all places $v$ of $\mathrm{F}$.

Proof. - We will prove Theorem 10.2 following closely the method of [Jac01]. Let $f^{\prime}=\bigotimes_{v} f_{v}^{\prime} \in \mathcal{S}\left(\mathrm{G}_{\mathbf{A}}^{\prime}\right)$ and $\Phi=\bigotimes \Phi_{v} \in \mathcal{S}\left(\mathrm{X}_{\mathbf{A}}\right)$ be matching functions, i.e., such that $\Phi_{v} \longleftrightarrow f_{v}^{\prime}$ for all $v$. For every $y \in \mathrm{X}_{\mathbf{A}}$ let $f^{y}=\bigotimes_{v} f_{v}^{y} \in \mathcal{S}\left(\mathrm{G}_{\mathbf{A}}\right)$ be such that $\left(f^{y}\right)_{y \in \mathrm{X}_{\mathbf{A}}}$ represents $\Phi$. (Definition as in the local case.) Let $\alpha^{\pi} \in \mathcal{E}_{\mathrm{G}_{\mathbf{A}}}\left(\mathrm{X}_{\mathbf{A}}, \pi^{*}\right)$ be given by

$$
\alpha_{x}^{\pi}(\phi)= \begin{cases}\mathcal{P}_{y}(\pi(g) \phi) & \text { if } x=y \bullet g, y \in \mathrm{X}, g \in \mathrm{G}_{\mathbf{A}}, \\ 0 & \text { otherwise. }\end{cases}
$$

It follows from the relative trace formula identity (see Theorem C.3) that

$$
\tilde{\mathfrak{B}}_{\mathfrak{A}_{\pi}}^{\alpha^{\pi}, \mathbf{W}_{e}^{\hat{e}}}(\Phi)=\mathbf{B}_{\pi^{\prime}}\left(f^{\prime}\right)+\mathbf{B}_{\pi^{\prime} \cdot \eta}\left(f^{\prime}\right) .
$$

Using the global analogue of $(\mathbf{2 . 1 1})$ we can write this identity as

$$
\sum_{y \in \mathrm{X} / \mathrm{G}} \mathfrak{B}_{\mathfrak{Q} \mathfrak{l}_{\pi}}^{\mathcal{P}_{j}, \mathbf{W}_{e}^{\hat{x}}}\left(f^{y}\right)=\mathbf{B}_{\pi^{\prime}}\left(f^{\prime}\right)+\mathbf{B}_{\pi^{\prime} \cdot \eta}\left(f^{\prime}\right)
$$

Fix $x \in \mathrm{X}$ and let $f \in \mathcal{S}\left(\mathrm{G}_{\mathbf{A}}\right)$. By Theorem 3.1 there exists $f^{\prime} \in \mathcal{S}\left(\mathrm{G}_{\mathbf{A}}^{\prime}\right)$ such that $f_{v}^{\prime} \in \mathcal{S}\left(\mathrm{G}_{v}^{\prime}[x]\right)$ for all $v$ and $f \stackrel{x}{\longleftrightarrow} f^{\prime}$. Since $x$ is a rational point, we have $f^{\prime}=(\eta \circ \operatorname{det}) f^{\prime}$. The factorization (10.2) together with (2.15) imply that $\mathbf{B}_{\pi^{\prime} \cdot \eta}\left(f^{\prime}\right)=\mathbf{B}_{\pi^{\prime}}\left(f^{\prime}\right)$. For any $y \in$ $x \bullet \mathrm{G}_{\mathbf{A}}$ choose $g \in \mathrm{G}_{\mathbf{A}}$ such that $y=x \bullet g$ and let $f^{y}=\mathrm{L}(g) f$. Set $f^{y}=0$ if $y \notin x \bullet \mathrm{G}_{\mathbf{A}}$. Then the family $\left(f^{y}\right)_{y \in \mathrm{X}_{\mathbf{A}}}$ represents a function $\Phi$ such that $\Phi \longleftrightarrow f^{\prime}$. It therefore follows from $(\mathbf{1 0 . 4})$ that

$$
\mathfrak{B}_{\mathfrak{A l}_{\pi}}^{\mathcal{P}_{x}, \mathbf{W}_{t}^{\hat{t}}}(f)=2 \mathbf{B}_{\pi^{\prime}}\left(f^{\prime}\right) .
$$

Assume first that $\pi$ is $\mathrm{G}^{x}$-distinguished and fix a place $v_{0}$. As in [Jac01, Lemma 1], we show that $\pi_{v_{0}}^{\prime} \in \mathcal{B I}_{x_{v_{0}}}$. By (a global analogue of) Lemma 2.2 there exists $f_{0}^{v_{0}}$ such that $f_{v_{0}} \mapsto \mathfrak{B}_{\mathfrak{A}_{\pi}}^{\mathcal{P}_{x}, \mathbf{W}_{c}^{\hat{t}}}\left(f_{v_{0}} \otimes f_{0}^{v_{0}}\right)$ is a non-zero distribution on $\mathbf{G}_{v_{0}}$. On the other hand, taking $f^{\prime}=f_{v_{0}}^{\prime} \otimes f_{0}^{\prime v_{0}}$ such that $f_{v_{0}} \otimes f_{0}^{v_{0}} \stackrel{x}{\longleftrightarrow} f^{\prime}$ as above, it follows from $(\mathbf{1 0 . 2})$ and $(\mathbf{1 0 . 5})$ that

$$
\mathfrak{B}_{\mathfrak{A}_{\pi}}^{\mathcal{P}_{\boldsymbol{T}}, \mathbf{W}_{i}^{\hat{t}}}\left(f_{v_{0}} \otimes f_{0}^{v_{0}}\right)=2 \mathbf{B}_{\pi^{\prime}}\left(f^{\prime}\right)=c \mathbf{B}_{\pi_{v_{0}}^{\prime}}\left(f_{v_{0}}^{\prime}\right)
$$

for some constant $c \neq 0$.

Define $\mathrm{A}_{x}: \mathcal{W}\left(\pi_{v_{0}}\right) \rightarrow \mathcal{W}\left(\pi^{v_{0}}\right)^{*}$ by

$$
\mathrm{A}_{x}(\mathrm{~W})(\xi)=\mathcal{P}_{x} \circ\left(\mathbf{W}^{\pi}\right)^{-1}(\mathrm{~W} \otimes \xi), \quad \mathrm{W} \in \mathcal{W}\left(\pi_{v_{0}}\right), \xi \in \mathcal{W}\left(\pi^{v_{0}}\right) .
$$


Note that $\mathrm{A}_{x} \circ \mathcal{W}\left(h, \pi_{v_{0}}\right)=\mathrm{A}_{x}$ for all $h \in \mathrm{G}_{v_{0}}^{x_{v_{0}}}$ since $\mathcal{P}_{x}$ is $\mathrm{G}_{v_{0}}^{x_{v_{0}}}$-invariant. Define $\ell_{0} \in$ $\mathcal{W}\left(\pi_{v_{0}}\right)^{*}$ by

$$
\ell_{0}(\mathrm{~W})=\delta_{e}^{\pi^{v_{0} \vee}} \circ \Lambda_{\mathfrak{W}\left(\pi^{v_{0}}\right)}\left(\mathrm{A}_{x}(\mathrm{~W}) \circ \mathcal{W}\left(f_{0}^{v_{0}}, \pi^{v_{0}}\right)\right)
$$

where we write $\mathfrak{W}(\pi)=\mathfrak{W}\left(\pi_{v_{0}}\right) \otimes \mathfrak{W}\left(\pi^{v_{0}}\right)$ and correspondingly $\Lambda_{\mathfrak{W}\left(\pi^{v_{0}}\right)}: \mathcal{W}\left(\pi^{v_{0}}\right)^{\vee} \rightarrow$ $\mathcal{W}\left(\pi^{v_{0} \vee}\right)$. Then $\ell_{0}$ is $\mathrm{G}_{v_{0}}^{x_{v_{0}}}$-invariant, since $\mathrm{A}_{x}$ is, and we have

$$
\mathfrak{B}_{\mathfrak{A}_{\pi}}^{\mathcal{P}_{x}, \mathbf{W}_{e}^{\hat{\imath}}}\left(f_{v_{0}} \otimes f_{0}^{v_{0}}\right)=\mathfrak{B}_{\mathfrak{W}\left(\pi_{v_{0}}\right)}^{\ell_{0}, \delta_{e} \bar{v}_{0}^{\vee}}\left(f_{v_{0}}\right)
$$

Together with (10.6), it follows that $\pi_{v_{0}}^{\prime} \in \mathcal{B I}_{x_{v_{0}}}$ and $\alpha_{x_{v_{0}}}^{\pi_{v_{0}}^{\prime}}=c^{-1} \ell_{0}$. This shows the last part of the Theorem.

By Lemma 3.9 we may define the linear form $\mathrm{P}$ on the space of $\pi$ by

$$
\mathrm{P}(\phi)=\prod_{v} \mathrm{~L}\left(1, \pi_{v}^{\prime} \times \pi_{v}^{\prime \vee} \cdot \eta_{v}\right)^{-1} \alpha_{x_{v}}^{\pi_{v}^{\prime}}\left(\mathrm{W}_{v}\right)
$$

whenever $\mathbf{W}^{\pi}(\phi)=\prod_{v} \mathbf{W}_{v}$. For matching functions $f \stackrel{x}{\longleftrightarrow} f^{\prime}$ as before, the identities $(\mathbf{1 0 . 2}),(\mathbf{1 0 . 5})$ and the defining property of $\alpha_{x}^{\pi_{v}^{\prime}}$ yield

$$
\mathfrak{B}_{\mathfrak{A}_{\pi}}^{\mathcal{P}_{x}, \mathbf{W}_{e}^{\hat{\tau}}}(f)=2\left(\mathfrak{r} l^{\prime \pi^{\prime}}\right)^{-1} \prod_{v} \mathfrak{r} \mathfrak{l}_{v}^{l_{v}^{\prime}} \mathfrak{B}_{\mathfrak{W}^{\psi_{v}}\left(\pi_{v}\right)}^{\alpha_{x}^{\pi_{v}^{\prime}}, \delta_{e}^{\hat{t}_{v}}}\left(f_{v}\right) .
$$

On the other hand, as in $(\mathbf{1 0 . 2})$, the analogue for $\mathrm{G}$ of the relation $(\mathbf{1 0 . 1})$ implies that

$$
\begin{aligned}
\mathfrak{B}_{\mathfrak{A}_{\pi}}^{\mathrm{P}, \mathbf{W}_{e}^{\hat{\pi}}}(f) & =\left(\mathfrak{r} \mathfrak{l}^{\pi}\right)^{-1} \prod_{v} \frac{\mathfrak{r} \mathfrak{r}_{v}^{\pi_{v}}}{\mathrm{~L}\left(1, \pi_{v}^{\prime} \times \pi_{v}^{\prime \vee} \cdot \eta_{v}\right)} \mathfrak{B}_{\mathfrak{W}^{\psi_{v}}\left(\pi_{v}\right)}^{\alpha^{\pi_{v}^{\prime}}, \delta_{e}^{\hat{\pi}_{v}}}\left(f_{v}\right) \\
& =\left(\mathfrak{r} \mathfrak{l}^{\pi}\right)^{-1} \prod_{v} \mathfrak{r} \mathfrak{l}_{v}^{\prime} \pi_{v}^{\prime} \mathfrak{B}_{\mathfrak{W}^{\psi_{v}}\left(\pi_{v}\right)}^{\alpha_{v}^{\pi_{v}^{v}}, \hat{\delta}_{e}^{\hat{v}_{v}}}\left(f_{v}\right) .
\end{aligned}
$$

Using these two equations we obtain that

$$
\mathfrak{B}_{\mathfrak{A}_{\pi}}^{\mathcal{P}_{x}, \mathbf{W}_{e}^{\hat{\tilde{t}}}}(f)=2 \mathrm{~L}\left(1, \pi^{\prime} \times \pi^{\prime \vee} \cdot \eta\right) \mathfrak{B}_{\mathfrak{A}_{\pi}^{\mathrm{P}}}^{\mathrm{P} \mathbf{W}_{e}^{\hat{\hat{t}}}}(f) .
$$

By (a global analogue of) Lemma 2.2 and the irreducibility of $\pi$ we infer $(\mathbf{1 0 . 3})$ and the Theorem under the assumption that $\pi$ is $G^{x}$-distinguished.

Finally, suppose that $\pi$ is not $\mathrm{G}^{x}$-distinguished. If there exists a place $v$ such that $\pi_{v}^{\prime} \notin \mathcal{B} \mathcal{I}_{x}$ then by definition $\alpha_{x}^{\pi_{v}^{\prime}} \equiv 0$ and therefore the identity $(\mathbf{1 0 . 3})$ is trivial. Otherwise $\pi_{v}^{\prime} \in \mathcal{B I}_{x_{v}}$ for all places $v$ of $\mathrm{F}$. The argument above is still applicable and shows $(\mathbf{1 0 . 3})$.

The factorization theorem immediately provides a criterion for distinction by any unitary group. 
Corollary 10.3. - Let $x \in \mathrm{X}$ and $\pi=\mathrm{bc}\left(\pi^{\prime}\right) \in \mathcal{C}^{\tau}$. Then

(1) $\pi$ is $\mathrm{G}^{x}$-distinguished if and only if $\alpha_{x}^{\pi_{v}^{\prime}} \not \equiv 0$ for every place $v$ of $\mathrm{F}$.

(2) In particular, if there exists a place $v$ of $\mathrm{F}$ such that $x \in \mathrm{X}_{v}^{-}$and $\pi_{v}^{\prime} \simeq \pi_{v}^{\prime} \cdot \eta_{v}$ then $\pi$ is not $\mathrm{G}^{x}$-distinguished.

(3) For the converse direction, suppose that $\mathrm{E} / \mathrm{F}$ splits at infinity, i.e., every real place of $\mathrm{F}$ splits in $\mathrm{E}$. Then $\pi$ is $\mathrm{G}^{x}$-distinguished (and consequently, $\pi_{v} \in \mathcal{B I}_{x}$ and $\alpha_{x}^{\pi_{v}} \not \equiv 0$ for all $v$ ) provided that for any place $v$ of $\mathrm{F}$ such that $x \notin \mathrm{X}_{v}^{+}$we have $\pi_{v}^{\prime} \neq \pi_{v}^{\prime} \cdot \eta_{v}$.

(4) Similarly, suppose that $n=2$ and $x \in \mathrm{X}_{v}^{+}$for all $v \mid \infty$ inert. Then $\pi$ is $\mathrm{G}^{x}$-distinguished (and consequently, $\pi_{v} \in \mathcal{B I}_{x}$ and $\alpha_{x}^{\pi_{v}} \not \equiv 0$ for all $v$ ) provided that for any finite place $v$ of $\mathrm{F}$ such that $x \notin \mathrm{X}_{v}^{+}$we have $\pi_{v}^{\prime} \not \pi_{v}^{\prime} \cdot \eta_{v}$.

Proof. - The first statement immediately follows from $(\mathbf{1 0 . 3})$. The second statement follows from Lemma 3.8. Now suppose that $\mathrm{E} / \mathrm{F}$ splits at infinity and $\pi$ is not $\mathrm{G}^{x}$ distinguished. For every $f^{\prime}=\bigotimes_{v} f_{v}^{\prime}$ with $f_{v}^{\prime} \in \mathcal{S}\left(\mathrm{G}_{v}^{\prime}[x]\right)$ there exists $f \stackrel{x}{\longleftrightarrow} f^{\prime}$. It follows from the global Bessel identity $(\mathbf{1 0 . 5})$ that $\mathbf{B}_{\pi^{\prime}}\left(f^{\prime}\right)=0$ for all such $f^{\prime}$. The factorization $(\mathbf{1 0 . 2})$ therefore implies that there exists a place $v$ such that $\left.\mathbf{B}_{\pi_{v}^{\prime}}\right|_{\mathcal{S}\left(\mathrm{G}_{v}^{\prime}[x]\right)} \equiv 0$. It now follows from Lemma 2.6 that $x \in \mathrm{X}_{v}^{-}$and $\pi_{v}^{\prime} \simeq \pi_{v}^{\prime} \cdot \eta_{v}$.

The same argument works in the case $n=2$ since $\mathrm{X}_{v}[x]=x \bullet \mathrm{G}_{v}$ for all $v \mid \infty$ by our assumption on $x$.

Remark 10.4. - In particular if $\mathrm{E} / \mathrm{F}$ splits at infinity and $n$ is odd then any $\pi \in \mathcal{C}^{\tau}$ is $\mathrm{G}^{x}$-distinguished for all $x \in \mathrm{X}$. Note that in this case $\mathrm{G}^{x}$ is automatically quasi-split. More generally, without an assumption on $\mathrm{E} / \mathrm{F}$ and $n$, Jacquet showed that any $\pi \in \mathcal{C}^{\tau}$ is $\mathrm{G}^{x}$-distinguished when $\mathrm{G}^{x}$ is quasi-split [Jac10]. In Corollary 12.9 we will further extend this to give an explicit criterion for the non-vanishing of $\alpha_{x}^{\pi_{v}^{\prime}}$.

\section{Factorization of unitary periods-Eisenstein series}

In this section we extend the global results of [LR00] and [Off07] from the case of Hecke characters of the diagonal torus to cuspidal automorphic representations on any Levi subgroup. We define global "open periods" and obtain their meromorphic continuation and functional equations. Using them we also obtain global Bessel identities.

11.1. Factorization of Bessel distributions for $\mathrm{G}^{\prime}$. - First we discuss the global analogue of the normalized Bessel distributions $\mathbf{B}\left(\sigma^{\prime}, \lambda\right)$ on $\mathrm{G}^{\prime}$.

Let $\mathrm{M}=\mathrm{M}_{\kappa}$ and $\sigma \in \mathcal{C}^{\mathrm{M}}$. Denote by $\mathcal{A}_{\sigma}=\mathcal{A}_{\mathrm{M}, \sigma}^{\mathrm{G}}$ the space of smooth functions $\varphi: \mathrm{U}_{\mathbf{A}} \mathrm{M} \backslash \mathrm{G}_{\mathbf{A}} \rightarrow \mathbf{C}$ such that for any $g \in \mathrm{G}_{\mathbf{A}}$ the function $\delta_{\mathrm{P}}^{-\frac{1}{2}} \varphi(\cdot g)$ on $\mathrm{M} \backslash \mathrm{M}_{\mathbf{A}}$ belongs to the space of $\sigma$. For $\varphi \in \mathcal{A}_{\sigma}$ we write $\varphi_{\lambda}=e^{\langle\lambda, \mathrm{H}(\cdot)\rangle} \varphi$ for any $\lambda \in \mathfrak{a}_{\mathrm{M}, \mathbf{C}}^{*}$. Let $\mathrm{I}(\sigma, \lambda)$ be the representation of $\mathrm{G}_{\mathbf{A}}$ on $\mathcal{A}_{\sigma}$ given by $[\mathrm{I}(g, \sigma, \lambda) \varphi]_{\lambda}(x)=\varphi_{\lambda}(x g), x, g \in \mathrm{G}_{\mathbf{A}}$. For $\varphi \in \mathcal{A}_{\sigma}$ 
denote by $\mathrm{E}(\varphi, \lambda)$ the Eisenstein series defined as the meromorphic continuation of the sum

$$
\mathrm{E}(g, \varphi, \lambda)=\sum_{\delta \in \mathrm{P} \backslash \mathrm{G}} \varphi_{\lambda}(\delta g)
$$

(Cf. [Lap08] for the meromorphic continuation in this context.) More generally, for $v \in$ $\mathcal{C}^{\mathrm{L}}, \varphi \in \mathcal{A}_{\mathrm{L}, v}^{\mathrm{G}}$ and $\lambda \in \mathfrak{a}_{\mathrm{L}, \mathbf{G}}^{*}$ we denote by $\mathrm{E}^{\mathrm{P}}(\varphi, \lambda)$ the meromorphic continuation of

$$
\mathrm{E}^{\mathrm{P}}(g, \varphi, \lambda)=\sum_{\delta \in \mathrm{Q} \backslash \mathrm{P}} \varphi_{\lambda}(\delta g) .
$$

The Fourier coefficient $\mathbf{W}(\varphi, \sigma, \lambda)$ of the Eisenstein series is given by (the meromorphic continuation of) the integral

$$
\begin{aligned}
\mathbf{W}(g: \varphi, \sigma, \lambda) & =\int_{\mathrm{U}_{0} \cap \overleftarrow{\mathrm{M}} \backslash\left(\mathrm{U}_{0}\right)_{\mathbf{A}}} \varphi_{\lambda}\left(w_{0}^{-1} u g\right) \psi_{0}^{-1}(u) d u \\
& =\int_{\mathrm{U}_{0} \cap \overleftarrow{\mathrm{M}} \backslash\left(\mathrm{U}_{0}\right)_{\mathbf{A}}} \varphi_{\lambda}\left(w_{\mathrm{M}}^{-1} u g\right) \psi_{0}^{-1}(u) d u
\end{aligned}
$$

We set

$$
\mathbf{W}_{e}(\varphi: \sigma, \lambda)=\mathbf{W}(e: \varphi, \sigma, \lambda)
$$

We write $\mathfrak{A}(\sigma, \lambda)$ for the triple $\left(\mathrm{I}(\sigma, \lambda), \mathrm{I}(\hat{\sigma},-\lambda),(\cdot, \cdot)_{\mathrm{AM}_{M} \mathrm{MU}_{\mathbf{A}} \backslash \mathrm{G}_{\mathbf{A}}}\right)$ where

$$
(\varphi, \hat{\varphi})_{\mathrm{A}_{\mathrm{M}} \mathrm{MU}_{\mathbf{A}} \backslash \mathrm{G}_{\mathbf{A}}}=\int_{\mathrm{A}_{\mathrm{M} M \mathrm{~A}_{\mathbf{A}} \backslash \mathrm{G}_{\mathbf{A}}}} \varphi(g) \hat{\varphi}(g) d g, \quad \varphi \in \mathcal{A}_{\sigma}, \hat{\varphi} \in \mathcal{A}_{\hat{\sigma}}
$$

Given $\sigma^{\prime}=\sigma_{1}^{\prime} \otimes \cdots \otimes \sigma_{t}^{\prime} \in \mathcal{C}^{\mathrm{M}^{\prime}}$ define

$(\mathbf{1 1 . 1})$

$$
\mathbf{B}\left(f^{\prime}: \sigma^{\prime}, \lambda\right)=\mathfrak{B}_{\mathfrak{A}\left(\sigma^{\prime}, \lambda\right)}^{\mathbf{W}\left(w_{0}: \sigma^{\prime}, \lambda\right), \mathbf{W}_{e}\left(\hat{\sigma}^{\prime},-\lambda\right)}\left(f^{\prime}\right), \quad f^{\prime} \in \mathcal{S}\left(\mathrm{G}_{\mathbf{A}}^{\prime}\right), \lambda \in \mathfrak{a}_{\mathrm{M}, \mathbf{G}}^{*} .
$$

As in the local case set

$$
\mathfrak{c s}\left(\sigma^{\prime}, \lambda\right)=\prod_{i<j} \mathrm{~L}\left(\lambda_{i}-\lambda_{j}+1, \sigma_{i}^{\prime} \times \sigma_{j}^{\prime \vee}\right) .
$$

We have a factorization

$$
\mathbf{W}\left(g: \varphi, \sigma^{\prime}, \lambda\right)=\mathfrak{c s}\left(\sigma^{\prime}, \lambda\right)^{-1} \prod_{v} \mathfrak{c} \mathfrak{s}_{v}\left(\sigma_{v}^{\prime}, \lambda\right) \mathbf{W}\left(g_{v}: \mathbf{W}_{\varphi_{v}}, \sigma_{v}^{\prime}, \lambda\right)
$$

for $\varphi=\bigotimes_{v} \varphi_{v} \in \mathcal{A}_{\sigma^{\prime}}$ and $\lambda \in \mathfrak{a}_{\mathrm{M}, \mathbf{C}}^{*}$ where $\mathrm{W}_{\varphi_{v}} \in \mathrm{I}_{\mathrm{M}}^{\mathrm{G}}\left(\mathcal{W}\left(\sigma_{v}^{\prime}\right)\right)$ are such that

(11.2) $\quad \mathbf{W}^{\mathrm{M}^{\prime}}(\mathrm{I}(g, \sigma, 0) \varphi)=\int_{\left(\mathrm{U}_{0}^{\prime} \cap \mathrm{M}^{\prime}\right) \backslash\left(\mathrm{U}_{0}^{\prime} \cap \mathrm{M}^{\prime}\right)_{\mathbf{A}}} \varphi(u g) \psi_{0}^{-1}(u) d u=\prod_{v} \mathrm{~W}_{\varphi_{v}}\left(g_{v}\right)(e), \quad g \in \mathrm{G}_{\mathbf{A}}^{\prime}$. 
Thus, if we define

$$
\mathrm{W}\left(g: \sigma^{\prime}, \lambda\right)=\mathfrak{c s}\left(\sigma^{\prime}, \lambda\right)^{-1} \bigotimes_{v} \mathfrak{c s}_{v}\left(\sigma_{v}^{\prime}, \lambda\right) \mathbf{W}\left(g_{v}: \sigma_{v}^{\prime}, \lambda\right)
$$

on $\mathrm{I}\left(\mathcal{W}\left(\sigma^{\prime}\right), \lambda\right)$ then using the above and the analogue of $(\mathbf{1 0 . 1})$ for $\mathrm{M}^{\prime}$ together with the global analogue of $(\mathbf{2 . 4})$ we get

$$
\mathbf{B}\left(f^{\prime}: \sigma^{\prime}, \lambda\right)=\mathfrak{B}_{\mathrm{I}\left(\mathfrak{W}\left(\sigma^{\prime}\right), \lambda\right)}^{\mathrm{W}\left(\sigma^{\prime}, \lambda\right), \mathrm{W}\left(e: \hat{\sigma}^{\prime},-\lambda\right)} .
$$

Therefore

$$
\mathbf{B}\left(f^{\prime}: \sigma^{\prime}, \lambda\right)=\mathfrak{r l}^{\prime}\left(\sigma^{\prime}, \lambda\right)^{-1} \prod_{v} \mathfrak{r} \mathfrak{l}^{\prime}\left(\sigma_{v}^{\prime}, \lambda\right) \mathbf{B}\left(f_{v}^{\prime}: \sigma_{v}^{\prime}, \lambda\right)
$$

where

$$
\begin{aligned}
\mathfrak{r l}^{\prime}\left(\sigma^{\prime}, \lambda\right) & =\mathfrak{r} \mathfrak{l}^{\prime \sigma^{\prime}} \prod_{i \neq j} \mathrm{~L}\left(\lambda_{i}-\lambda_{j}+1, \sigma_{i}^{\prime} \times \sigma_{j}^{\prime \vee}\right) \\
& =\mathfrak{r} \mathfrak{l}^{\prime \sigma^{\prime}} \mathfrak{c s}(\sigma, \lambda) \mathfrak{c s}\left(\sigma^{\vee},-\lambda\right), \quad \mathfrak{r} l^{\prime \sigma^{\prime}}=\prod_{i=1}^{t} \mathfrak{r} \mathfrak{l}^{\sigma_{i}^{\prime}}
\end{aligned}
$$

and

$$
\begin{aligned}
\mathfrak{r l}_{v}^{\prime}\left(\sigma_{v}^{\prime}, \lambda\right) & =\mathrm{L}\left(1, \mathrm{I}\left(\sigma_{v}^{\prime}, \lambda\right) \times \mathrm{I}\left(\sigma_{v}^{\prime \vee},-\lambda\right)\right) \\
& =\prod_{i, j} \mathrm{~L}\left(\lambda_{i}-\lambda_{j}+1,\left(\sigma_{i}^{\prime}\right)_{v} \times\left(\sigma_{j}^{\prime}\right)_{v}^{\vee}\right) \\
& =\mathfrak{r} \mathfrak{l}_{v}^{\prime \sigma_{v}^{\prime}} \mathfrak{c s}\left(\sigma_{v}, \lambda\right) \mathfrak{c s}\left(\sigma_{v}^{\vee},-\lambda\right) .
\end{aligned}
$$

11.2. Periods of Eisenstein series and Fourier inversion. - We will now apply Fourier inversion in the spirit of the stabilization of the elliptic part of the trace formula of SL(2) due to Labesse-Langlands [LL79] to write the (regularized) unitary period of any cuspidal Eisenstein series as a finite sum of factorizable invariant linear forms.

Let $\Xi=\mathrm{F}^{*} / \mathrm{Nm}\left(\mathrm{E}^{*}\right)$ and for every place $v$ of $\mathrm{F}$ let $\boldsymbol{\Xi}_{v}=\mathrm{F}_{v}^{*} / \mathrm{Nm}\left(\mathrm{E}_{v}^{*}\right)$. Thus $\boldsymbol{\Xi}_{v}$ is a group of order 2 with dual group $\Xi_{v}^{\vee}=\left\{1_{\mathrm{F}_{v}^{*}}, \eta_{v}\right\}$ if $v$ is inert and $\boldsymbol{\Xi}_{v}$ is the trivial group if $v$ is split. By class field theory, $\Xi$ embeds in $\Xi_{\mathbf{A}}:=\bigoplus_{v} \Xi_{v}=\mathbf{A}^{*} / \operatorname{Nm}\left(\mathbf{A}_{\mathrm{E}}^{*}\right)$ and the cokernel is a group of order 2 whose dual $\left(\boldsymbol{\Xi}_{\mathbf{A}} / \boldsymbol{\Xi}\right)^{\vee}=\left\{1_{\mathbf{A}^{*}}, \eta\right\}$ is the subgroup of $\boldsymbol{\Xi}_{\mathbf{A}}^{\vee}$ consisting of the characters of $\boldsymbol{\Xi}_{\mathbf{A}}$ that are trivial on $\boldsymbol{\Xi}$.

Fix a positive integer $t$. Let $f \in \mathrm{L}^{1}\left(\Xi_{\mathbf{A}}^{t}\right)$ (with respect to the counting measure) and let $\hat{f}$ be the Fourier transform defined by

$$
\hat{f}(\nu)=\sum_{\xi \in \Xi_{\mathbf{A}}^{t}} v(\xi) f(\xi), \quad v \in\left(\Xi_{\mathbf{A}}^{\vee}\right)^{t}=\left(\Xi_{\mathbf{A}}^{t}\right)^{\vee} .
$$


Similarly we define the Fourier transform in the local case with respect to $\Xi_{v}^{t}$. The following is a simple consequence of a finite Fourier inversion formula (cf. [LR03, Lemma 2]).

Lemma 11.1. - Let $f \in \mathrm{L}^{1}\left(\boldsymbol{\Xi}_{\mathbf{A}}^{t}\right)$. Then

$$
2^{t} \sum_{\xi \in \Xi^{t}} f(\xi)=\sum_{v \in\left(\left(\Xi_{\mathbf{A}} / \Xi\right)^{\vee}\right)^{t}} \hat{f}(v) .
$$

Furthermore, if there are functions $f_{v}$ on $\boldsymbol{\Xi}_{v}^{t}$ such that for any $\xi=\left(\xi_{v}\right)_{v} \in \boldsymbol{\Xi}_{\mathbf{A}}^{t}$ the product $\prod_{v} f_{v}\left(\xi_{v}\right)$ converges to $f(\xi)$ then for all $v \in\left(\left(\boldsymbol{\Xi}_{\mathbf{A}} / \boldsymbol{\Xi}\right)^{\vee}\right)^{t} \hat{f}(v)$ is given by the convergent product $\prod_{v} \hat{f}_{v}\left(v_{v}\right)$ where $v_{v}$ is the restriction of $v$ to $\boldsymbol{\Xi}_{v}^{t}$.

By abuse of notation we also treat an element $v=\left(v_{1}, \ldots, v_{t}\right) \in\left(\Xi_{\mathbf{A}}^{\vee}\right)^{t}$ as a character of $\mathbf{M}_{\mathbf{A}}^{\prime}$ or alternatively as a function on $\mathrm{X}_{\mathbf{A}} \cap \mathrm{M}_{\mathbf{A}}$ via

$$
v(y)=\prod_{i=1}^{t} v_{i}\left(\operatorname{det} y_{i}\right), \quad y=\operatorname{diag}\left(y_{1}, \ldots, y_{t}\right), y_{i} \in \mathrm{X}_{n_{i}} \text { or } y_{i} \in \mathrm{G}_{n_{i}}^{\prime} .
$$

Similarly, for the local components. We also set for convenience

$$
\eta v=\left(\eta v_{1}, \ldots, \eta v_{t}\right)
$$

Now let $\sigma \in \mathcal{C}^{\mathrm{M}, \tau}$. The fiber $\mathcal{B}(\sigma)$ of quadratic base change at $\sigma$ is a torsor of the group $\left(\left(\boldsymbol{\Xi}_{\mathbf{A}} / \Xi\right)^{\vee}\right)^{t}$. Thus, fixing a base point $\sigma^{\prime}=\sigma_{1}^{\prime} \otimes \cdots \otimes \sigma_{t}^{\prime} \in \mathcal{B}(\sigma)$ we have

$$
\mathcal{B}(\sigma)=\left\{\sigma^{\prime} \cdot v: v \in\left(\left(\Xi_{\mathbf{A}} / \Xi\right)^{\vee}\right)^{t}\right\}
$$

where we set $\sigma^{\prime} \cdot v=\sigma_{1}^{\prime} \cdot v_{1} \otimes \cdots \otimes \sigma_{t}^{\prime} \cdot v_{t}$.

Recall that by Theorem 10.2 the unitary periods, factorize compatibly on $\sigma$. More precisely, for any factorizable automorphic form $\phi$ in the space of $\sigma$ we have

$$
\int_{\mathrm{M}^{\vee} \backslash \mathrm{M}_{\mathbf{A}}^{\prime}} \phi(m) d m=2^{t}\left[\prod_{i=1}^{t} \mathrm{~L}\left(1, \sigma_{i}^{\prime} \times \sigma_{i}^{\prime \vee} \cdot \eta\right)\right] \prod_{v} \tilde{\alpha}_{y}^{\sigma_{v}^{\prime}}\left(\mathrm{W}_{v}\right)
$$

where

$$
\mathbf{W}^{\sigma}(\phi)=\prod_{v} \mathrm{~W}_{v}, \quad \mathrm{~W}_{v} \in \mathcal{W}^{\psi_{v}}\left(\sigma_{v}\right)
$$

and

$$
\tilde{\alpha}_{y}^{\sigma_{v}^{\prime}}=\left[\prod_{i=1}^{t} \mathrm{~L}\left(1,\left(\sigma_{i}^{\prime}\right)_{v} \times\left(\sigma_{i}^{\prime \vee}\right)_{v} \cdot \eta_{v}\right)^{-1}\right] \alpha_{y}^{\sigma_{v}^{\prime}}
$$


For $x \in \mathrm{X}, \sigma \in \mathcal{C}^{\mathrm{M}}$ and $\varphi \in \mathcal{A}_{\sigma}$ the intertwining period

$$
\mathrm{J}(\varphi: x, \sigma, \lambda)=\sum_{y \in\left(\mathrm{X}^{\mathrm{M} \cap x \bullet \mathrm{G}) / \mathrm{M}}\right.} \int_{\mathrm{M}^{y} \backslash \mathrm{G}_{\mathbf{A}}^{y}} \varphi_{\lambda}\left(g l_{y}^{x}\right) d g
$$

is defined by an absolutely convergent sum-integral for $\lambda \in \mathfrak{a}_{\mathrm{M}, \mathbf{C}}^{*}$ with $\operatorname{Re} \lambda$ sufficiently regular in the positive Weyl chamber (cf. [LR03, Theorem 5.2.1 and Lemma 5.2.1(2)]). Here, as before, $\iota_{y}^{x} \in \mathrm{G}$ is such that $x=y \bullet \iota_{y}^{x}$. Note that $\mathrm{J}(\varphi: x, \sigma, \lambda)$ vanishes unless there exists $y \in \mathrm{X}^{\mathrm{M}} \cap x \bullet \mathrm{G}$ such that $\sigma$ is $\mathrm{M}^{y}$-distinguished, and in particular, $\sigma \in \mathcal{C}^{\mathrm{M}, \tau}$. Moreover, in [LR03] the regularized period integral

$$
\mathcal{P}_{x}(\phi)=\int_{\mathrm{G}^{x} \backslash \mathrm{G}_{\mathbf{A}}^{x}}^{*} \phi(g) d g
$$

is defined for an automorphic form $\phi$ on $G \backslash G_{\mathbf{A}}$ under a certain open condition on the exponents of $\phi$ and by [ibid., Theorem 9.1.1] we have

$$
\mathcal{P}_{x}(\mathrm{E}(\varphi, \lambda))=\mathrm{J}(\varphi: x, \sigma, \lambda)
$$

for $\varphi \in \mathcal{A}_{\sigma}$ and $\operatorname{Re} \lambda$ sufficiently positive. By [Lap06, p. 290] $\mathcal{P}_{x} \circ \mathrm{E}(\cdot, \lambda) \in \mathrm{I}(\sigma, \lambda)^{*}$.

The infinite sum on the right-hand side of $(\mathbf{1 1 . 7})$ can be viewed as a sum over the P-orbits in the intersection of the G-orbit of $x$ with (the F-points of the unique Zariski open $\mathbf{P}$-orbit in $\mathbf{X}$. The individual summands are (factorizable) $\mathrm{G}_{\mathbf{A}}^{x}$-invariant linear forms, defined for Re $\lambda \gg 0$ by convergent integrals, but are not expected to admit a meromorphic continuation. We will show that, in fact, the resulting sum can be written as a finite sum of factorizable terms. To that end, we use the local invariant functionals defined in Section 4 to define for any $\sigma \in \mathcal{C}^{\mathrm{M}, \tau}, \sigma^{\prime}=\sigma_{1}^{\prime} \otimes \cdots \otimes \sigma_{t}^{\prime} \in \mathcal{B}(\sigma)$, a factorizable element $\varphi=\bigotimes_{v} \varphi_{v} \in \mathcal{A}_{\sigma}$ and $x \in \mathrm{X}_{\mathbf{A}}$

$$
\begin{aligned}
\mathrm{J}^{\mathrm{st}}\left(\varphi: x, \sigma^{\prime}, \lambda\right)= & {\left[\prod_{i=1}^{t} \mathrm{~L}\left(1, \sigma_{i}^{\prime} \times \sigma_{i}^{\prime \vee} \cdot \eta\right)\right] } \\
& \times \prod_{v}\left[\prod_{i=1}^{t} \mathrm{~L}\left(1,\left(\sigma_{i}^{\prime}\right)_{v} \times\left(\sigma_{i}^{\prime}\right)_{v}^{\vee} \cdot \eta_{v}\right)^{-1}\right] \\
& \times \mathrm{J}\left(\mathrm{W}_{\varphi_{v}}: x_{v}, \alpha^{\sigma_{v}^{\prime}}, \lambda\right)
\end{aligned}
$$

where $\mathrm{W}_{\varphi_{v}} \in \mathrm{I}_{\mathrm{M}}^{\mathrm{G}}\left(\mathcal{W}\left(\sigma_{v}\right)\right)$ are as in $(\mathbf{1 1 . 2})$. It follows from Proposition 4.1 and Lemma 8.2 that the product defining $\mathrm{J}^{\mathrm{st}}\left(\varphi: x, \sigma^{\prime}, \lambda\right)$ converges for $\operatorname{Re} \lambda \gg 0$ and admits a meromorphic continuation to $\lambda \in \mathfrak{a}_{\mathrm{M}, \mathbf{G}}^{*}$. In fact, whenever holomorphic at $\lambda$ the map $x \mapsto$ $\mathrm{J}^{\text {st }}\left(x, \sigma^{\prime}, \lambda\right)$ is an element of $\mathcal{E}_{\mathrm{G}_{\mathbf{A}}}\left(\mathrm{X}_{\mathbf{A}}, \mathrm{I}(\sigma, \lambda)^{*}\right)$ which we denote by $\mathrm{J}^{\text {st }}\left(\sigma^{\prime}, \lambda\right)$.

We also write

$$
\mathrm{J}^{\mathrm{st}}\left(\varphi: x, \sigma^{\prime}, \lambda\right)=\mathrm{j}\left(\sigma^{\prime}, \lambda\right) \prod_{v} \mathrm{j}_{v}\left(\sigma_{v}^{\prime}, \lambda_{v}\right)^{-1} \mathrm{~J}\left(\mathrm{~W}_{\varphi_{v}}: x_{v}, \alpha^{\sigma_{v}^{\prime}}, \lambda\right)
$$


where

$$
\begin{aligned}
\mathfrak{j}\left(\sigma^{\prime}, \lambda\right)= & {\left[\prod_{i=1}^{t} \mathrm{~L}\left(1, \sigma_{i}^{\prime} \times \sigma_{i}^{\prime \vee} \cdot \eta\right)\right] } \\
& \times \prod_{i<j} \frac{\mathrm{L}\left(\lambda_{i}-\lambda_{j}, \sigma_{i}^{\prime} \times \sigma_{j}^{\prime \vee} \cdot \eta\right)}{\varepsilon\left(\lambda_{i}-\lambda_{j}, \sigma_{i}^{\prime} \times \sigma_{j}^{\prime \vee} \cdot \eta\right) \mathrm{L}\left(1+\lambda_{i}-\lambda_{j}, \sigma_{i}^{\prime} \times \sigma_{j}^{\prime \vee}\right)}
\end{aligned}
$$

and

$$
\begin{aligned}
\mathfrak{j}_{v}\left(\sigma_{v}^{\prime}, \lambda_{v}\right)=\mathfrak{j}_{v}^{\psi_{v}^{\prime}}\left(\sigma_{v}^{\prime}, \lambda_{v}\right)= & {\left[\prod_{i=1}^{t} \mathrm{~L}\left(1,\left(\sigma_{i}^{\prime}\right)_{v} \times\left(\sigma_{i}^{\prime}\right)_{v}^{\vee} \cdot \eta_{v}\right)\right] } \\
\times & \mathrm{L}\left(\lambda_{i}-\lambda_{j},\left(\sigma_{i}^{\prime}\right)_{v} \times\left(\sigma_{j}^{\prime}\right)_{v}{ }^{\vee} \cdot \eta_{v}\right) \\
& \frac{\prod_{i<j}}{\varepsilon\left(\lambda_{i}-\lambda_{j},\left(\sigma_{i}^{\prime}\right)_{v} \times\left(\sigma_{j}^{\prime}\right)_{v}{ }^{\vee} \cdot \eta_{v} ; \psi_{v}^{\prime}\right) \mathrm{L}\left(1+\lambda_{i}-\lambda_{j},\left(\sigma_{i}^{\prime}\right)_{v} \times\left(\sigma_{j}^{\prime}\right)_{v}{ }^{\vee}\right)} .
\end{aligned}
$$

Recall that by Lemma 8.2 almost all the factors in $(\mathbf{1 1 . 8})$ are 1.

Theorem 11.2. - Let $\sigma \in \mathcal{C}^{\mathrm{M}, \tau}, \varphi \in \mathcal{A}_{\sigma}$ and $x \in \mathrm{X}$. Then as meromorphic functions in $\lambda$ we have

$$
\mathcal{P}_{x}(\mathrm{E}(\varphi, \lambda))=\mathrm{J}(\varphi: x, \sigma, \lambda)=\sum_{\sigma^{\prime} \in \mathcal{B}(\sigma)} \mathrm{J}^{\mathrm{st}}\left(\varphi: x, \sigma^{\prime}, \lambda\right)
$$

Proof. - We prove the identity for $\operatorname{Re} \lambda \gg 0$. The Theorem then follows by meromorphic continuation. Let $\mathrm{S}_{\infty}$ be the set of inert real places of $\mathrm{F}$. For every place $v$ of $\mathrm{F}$ the determinant map induces a surjection $\mathrm{X}_{v} / \mathrm{G}_{v} \rightarrow \boldsymbol{\Xi}_{v}$ and the map is a bijection if and only if $v \notin \mathrm{S}_{\infty}$. The Hasse principle for Hermitian forms implies that for any $x \in \mathrm{X}$ we have

$$
x \bullet \mathrm{G}_{\mathbf{A}} \cap \mathrm{X}=x \bullet \mathrm{G}
$$

and furthermore, $\mathrm{X} \bullet \mathrm{G}_{\mathbf{A}}=\mathrm{X}_{\mathbf{A}} \cap \operatorname{Ker} \eta$, an open subspace of $\mathrm{X}_{\mathbf{A}}$. It follows that $\mathrm{X} / \mathrm{G}$ is in bijection with the following subset of $\left(\mathrm{X}_{\mathrm{S}_{\infty}} / \mathrm{G}_{\mathrm{S}_{\infty}}\right) \times \Xi$ :

$$
\Omega_{\mathrm{G}}=\left\{\left(z \bullet \mathrm{G}_{\mathrm{S}_{\infty}}, \xi\right): z \in \mathrm{X}_{\mathrm{S}_{\infty}}, \xi \in \Xi, \operatorname{det} z_{v} \in \xi \text { for all } v \in \mathrm{S}_{\infty}\right\}
$$

Denote the bijection by $\iota_{\mathrm{G}}: \mathrm{X} / \mathrm{G} \rightarrow \Omega_{\mathrm{G}}$. Then $\iota_{\mathrm{G}}(x)=\left(x \bullet \mathrm{G}_{\mathrm{S}_{\infty}}\right.$, $\left.\operatorname{det} x \mathrm{Nm}\left(\mathrm{E}^{*}\right)\right)$. Similarly, the assignment

$$
\operatorname{diag}\left(y_{1}, \ldots, y_{t}\right) \mapsto\left(\operatorname{det} y_{1}, \ldots, \operatorname{det} y_{t}\right)
$$

defines surjective maps $d_{v}: \mathrm{X}_{v}^{\mathrm{M}_{v}} / \mathrm{M}_{v} \rightarrow \Xi_{v}^{t}$ for every place $v$ of $\mathrm{F}$ and $d: \mathrm{X}^{\mathrm{M}} / \mathrm{M} \rightarrow \Xi^{t}$. Again $d_{v}$ is bijective if $v \notin \mathrm{S}_{\infty}$ and $d$ is finite-to-one. We set 


$$
\begin{gathered}
\Omega_{\mathrm{M}}=\left\{\left(z \bullet \mathrm{M}_{\mathrm{S}_{\infty}}, \xi\right): z \in \mathrm{X}_{\mathrm{S}_{\infty}} \cap \mathrm{M}_{\mathrm{S}_{\infty}}, \xi \in \Xi^{t},\right. \\
\left.d_{v}\left(z_{v} \bullet \mathrm{M}_{v}\right)=\xi \text { for all } v \in \mathrm{S}_{\infty}\right\}
\end{gathered}
$$

and let $\iota_{\mathrm{M}}: \mathrm{X}^{\mathrm{M}} / \mathrm{M} \rightarrow \Omega_{\mathrm{M}}$ be the associated bijection. Note that for any $\xi \in \Xi^{t}$ we have

$$
\iota_{\mathrm{M}}\left(d^{-1}(\xi)\right)=\left\{(z, \xi): z \in\left(\mathrm{X}_{\mathrm{S}_{\infty}} \cap \mathrm{M}_{\mathrm{S}_{\infty}}\right) / \mathrm{M}_{\mathrm{S}_{\infty}}, d_{v}\left(z_{v}\right)=\xi \text { for all } v \in \mathrm{S}_{\infty}\right\}
$$

Fix $\sigma^{\prime} \in \mathcal{B}(\sigma)$, a factorizable section $\varphi=\bigotimes_{v} \varphi_{v} \in \mathcal{A}_{\sigma}$ and $\operatorname{Re} \lambda \gg 0$. For $y \in$ $\mathrm{X}_{v}^{\mathrm{M}_{v}} / \mathrm{M}_{v}$ let

$$
h_{v}(y)=\left\{\begin{array}{l}
v_{\mathrm{M}_{v}}(y) e^{\frac{1}{2}\left\langle\rho_{\mathrm{M}_{v}}+\lambda, \mathrm{H}(y)\right\rangle} \int_{\mathrm{M}_{v}^{y} \backslash \mathrm{G}_{v}^{y}} \tilde{\alpha}_{y}^{\sigma_{v}^{\prime}}\left(\left(\mathrm{W}_{\varphi_{v}}\right)_{\lambda}\left(g l_{y}^{x}\right)\right) d g \\
\text { if } y \in\left(x \bullet \mathrm{G}_{v} \cap \mathrm{M}_{v}\right) / \mathrm{M}_{v} \\
0 \quad \text { otherwise }
\end{array}\right.
$$

where $\tilde{\alpha}_{y}^{\sigma_{v}^{\prime}}$ is defined by $(\mathbf{1 1 . 6})$. For $\xi \in \mathbf{\Xi}_{v}^{t}$ let

$$
f_{v}(\xi)=\sum_{y \in d_{v}^{-1}(\xi)} h_{v}(y)
$$

In particular, for $v \notin \mathrm{S}_{\infty}$ we have $h_{v}=f_{v} \circ d_{v}$. Set $f^{\mathrm{S}_{\infty}}=\prod_{v \notin \mathrm{S}_{\infty}} f_{v}$ and $f=\prod_{v} f_{v}$. It follows from [LR03, Theorem 5.2.1] that the product is absolutely convergent and that $f \in \mathrm{L}^{1}\left(\boldsymbol{\Xi}_{\mathbf{A}}^{t}\right)$.

We get from $(\mathbf{1 1 . 5})$ and $(\mathbf{1 1 . 7})$ that

$$
\mathrm{J}(\varphi: x, \sigma, \lambda)=2^{t}\left[\prod_{i=1}^{t} \mathrm{~L}\left(1, \sigma_{i}^{\prime} \times \sigma_{i}^{\prime \vee} \cdot \eta\right)\right] \sum_{y \in\left(\mathrm{X}^{\mathrm{M} \cap{ }^{\prime}} \in \mathrm{G}\right) / \mathrm{M}} \prod_{v} h_{v}(y) .
$$

Summing in stages we have

$$
\sum_{y \in\left(\mathrm{X}^{\mathrm{M} \cap x} \bullet \mathrm{G}\right) / \mathrm{M}} \prod_{v} h_{v}(y)=\sum_{\xi \in \Xi^{t}} f^{\mathrm{S}_{\infty}}(\xi) \sum_{y \in d^{-1}(\xi)} \prod_{v \in \mathrm{S}_{\infty}} h_{v}(y) .
$$

Applying (11.10) we get that

$$
\sum_{y \in d^{-1}(\xi)} \prod_{v \in \mathrm{S}_{\infty}} h_{v}(y)=\sum_{z \in\left(\mathrm{X}_{\mathrm{S}_{\infty}} \cap \mathrm{M}_{\mathrm{S}_{\infty}}\right) / \mathrm{M}_{\mathrm{S}_{\infty}}} \prod_{v \in \mathrm{S}_{\infty}} \mathbf{1}_{d_{v}^{-1}(\xi)}\left(z_{v}\right) h_{v}\left(z_{v}\right)=\prod_{v \in \mathrm{S}_{\infty}} f_{v}(\xi)
$$

and therefore

$$
\mathrm{J}(\varphi: x, \sigma, \lambda)=2^{t}\left[\prod_{i=1}^{t} \mathrm{~L}\left(1, \sigma_{i}^{\prime} \times \sigma_{i}^{\prime \vee} \cdot \eta\right)\right] \sum_{\xi \in \Xi^{t}} f(\xi) .
$$


It now follows from Lemma 11.1 that

$$
\mathrm{J}(\varphi: x, \sigma, \lambda)=\left[\prod_{i=1}^{t} \mathrm{~L}\left(1, \sigma_{i}^{\prime} \times \sigma_{i}^{\prime \vee} \cdot \eta\right)\right]_{v \in\left(\left(\Xi_{\mathbf{A}} / \Xi\right)^{\vee}\right)^{t}} \prod_{v} \hat{f}_{v}\left(v_{v}\right)
$$

and the product on the right-hand side converges. Finally, we observe that by $(\mathbf{3 . 1 3})$ we have

$$
\tilde{\alpha}_{y}^{\sigma_{v}^{\prime} \cdot v_{v}}=v_{v}\left(w_{0}^{\mathrm{M}} y\right) \tilde{\alpha}_{y}^{\sigma_{v}^{\prime}}, y \in \mathrm{X}_{v}^{\mathrm{M}_{v}}
$$

and therefore

$$
\hat{f}_{v}\left(v_{v}\right)=v_{v}\left(w_{0}^{\mathrm{M}}\right) \mathrm{J}\left(\varphi_{v}: x, \tilde{\alpha}^{\sigma_{v}^{\prime} \cdot v_{v}}, \lambda\right) .
$$

Since globally $v\left(w_{0}^{\mathrm{M}}\right)=1$ we get that

$$
\prod_{v} \hat{f}_{v}\left(v_{v}\right)=\prod_{v} \mathrm{~J}\left(\varphi_{v}: x, \tilde{\alpha}^{\sigma_{v}^{\prime} \cdot v_{v}}, \lambda\right)
$$

Together with $(\mathbf{1 1 . 4})$ and the fact that $\prod_{i=1}^{t} \mathrm{~L}\left(1, \sigma_{i}^{\prime} \times \sigma_{i}^{\prime \vee} \cdot \eta\right)$ is independent of $\sigma^{\prime} \in \mathcal{B}(\sigma)$, this gives $(\mathbf{1 1 . 9})$ for $\operatorname{Re} \lambda \gg 0$.

11.3. Factorization of Bessel distributions. - We retain the notation of the previous section.

Observe that by applying the functional equations

$$
\mathrm{L}\left(\lambda_{i}-\lambda_{j}, \sigma_{i}^{\prime} \times \sigma_{j}^{\prime \vee} \cdot \eta\right)=\varepsilon\left(\lambda_{i}-\lambda_{j}, \sigma_{i}^{\prime} \times \sigma_{j}^{\prime \vee} \cdot \eta\right) \mathrm{L}\left(1-\lambda_{i}+\lambda_{j}, \sigma_{i}^{\prime \vee} \times \sigma_{j}^{\prime} \cdot \eta\right)
$$

we get

$$
\begin{aligned}
\frac{\mathfrak{j}\left(\sigma^{\prime}, \lambda\right)}{\mathfrak{c s}\left(\sigma^{\vee},-\lambda\right) \mathfrak{r} l^{\sigma}} & =\frac{\left[\prod_{i=1}^{t} \mathrm{~L}\left(1, \sigma_{i}^{\prime} \times \sigma_{i}^{\prime \vee} \cdot \eta\right)\right]\left[\prod_{1 \leq i<j \leq t} \frac{\mathrm{L}\left(1-\lambda_{i}+\lambda_{j}, \sigma_{i}^{\prime \vee} \times \sigma_{j}^{\prime} \cdot \eta\right)}{\mathrm{L}\left(1+\lambda_{i}-\lambda_{j}, \sigma_{i}^{\prime} \times \sigma_{j}^{\prime \prime}\right)}\right]}{\mathfrak{r} \mathfrak{l}^{\sigma} \mathfrak{c s}\left(\sigma^{\vee},-\lambda\right)} \\
& =\mathfrak{r} \mathfrak{l}^{\prime}\left(\sigma^{\prime}, \lambda\right)^{-1} .
\end{aligned}
$$

Similarly, for all $v$

$$
\frac{\mathfrak{j}_{v}\left(\sigma_{v}^{\prime}, \lambda\right)}{\mathfrak{c s}_{v}\left(\sigma_{v}^{\vee},-\lambda\right) \mathfrak{r} \mathfrak{l}_{v}^{\sigma_{v}}} \mathfrak{n}\left(\sigma_{v}^{\prime}, \lambda\right)=\mathfrak{r l}_{v}^{\prime}\left(\sigma_{v}^{\prime}, \lambda\right)^{-1}
$$

Using (11.11) and the discussion of the previous section we now obtain a factorization of

$$
\tilde{\mathbf{B}}\left(\sigma^{\prime}, \lambda\right):=\tilde{\mathfrak{B}}_{\mathfrak{A}(\sigma, \lambda)}^{\mathrm{st}^{\mathrm{st}}\left(\sigma^{\prime}, \lambda\right), \mathbf{W}_{e}(\hat{\sigma},-\lambda)}
$$


as

$(\mathbf{1 1 . 1 2})$

$$
\mathfrak{r l}^{\prime}\left(\sigma^{\prime}, \lambda\right)^{-1} \prod_{v} \mathfrak{r} \mathfrak{r}_{v}^{\prime}\left(\sigma_{v}^{\prime}, \lambda\right) \tilde{\mathbf{B}}\left(\sigma_{v}^{\prime}, \lambda\right)
$$

where $\tilde{\mathbf{B}}\left(\sigma_{v}^{\prime}, \lambda\right)$ is defined in $(\mathbf{4 . 1 3})$ and for almost all $v$ we have $\mathfrak{r l}_{v}^{\prime}\left(\sigma_{v}^{\prime}, \lambda\right) \tilde{\mathbf{B}}\left(\mathbf{1}_{\mathrm{X}_{v} \cap \mathrm{K}_{v}}\right.$ : $\left.\sigma_{v}^{\prime}, \lambda\right)=1$.

It now follows from Theorem 11.2 and the definition of $\alpha_{x}^{(\mathrm{M}, \sigma)}$ in $(\mathbf{C .} \mathbf{. 1})$ that

$$
\alpha_{x}^{(\mathrm{M}, \sigma)}(\varphi, \lambda)= \begin{cases}\sum_{\sigma^{\prime} \in \mathcal{B}(\sigma)} \mathrm{J}^{\mathrm{st}}\left(\varphi: x, \sigma^{\prime}, \lambda\right) & x \in \mathrm{X} \bullet \mathrm{G}_{\mathbf{A}} \\ 0 & \text { otherwise }\end{cases}
$$

In other words, we have

$$
\tilde{\mathbf{B}}(\sigma, \lambda)=\sum_{\sigma^{\prime} \in \mathcal{B}(\sigma)} \tilde{\mathbf{B}}\left(\sigma^{\prime}, \lambda\right)
$$

where the left-hand side is defined in Appendix C. We claim that for any $\sigma \in \mathcal{C}^{\mathrm{M}, \tau}$ we have

$$
\sum_{\sigma^{\prime} \in \mathcal{B}(\sigma)} \tilde{\mathbf{B}}\left(\sigma^{\prime}, \lambda\right) \longleftrightarrow \sum_{\sigma^{\prime} \in \mathcal{B}(\sigma)} \mathbf{B}\left(\sigma^{\prime}, \lambda\right)
$$

Indeed, it follows from Theorem C.3 that this holds up to a constant $c$ depending only on $\kappa$. Choosing an everywhere unramified quadratic extension $\mathrm{E} / \mathrm{F}$ that splits at infinity and at the even places and $\sigma \in \mathcal{C}^{\mathrm{M}, \tau}$ everywhere unramified, it follows from the local identities provided by Proposition 7.5 in the split places and $(\mathbf{8 . 5})$ for unramified data together with Remark 9.3 that $c=1$.

Assume now that $\mathrm{E} / \mathrm{F}$ splits at infinity. Then we can view the summands on the left-hand side of $(\mathbf{1 1 . 1 3})$ as distributions on $\mathrm{G}_{\mathbf{A}}^{\prime}$ via smooth matching. Let $\mathrm{S}$ be a finite set of places of $\mathrm{F}$ containing the Archimedean and the finite places with ramified data with respect to either $\mathrm{E} / \mathrm{F}, \psi^{\prime}$ or $\sigma$. Fixing any $f_{\mathrm{S}}^{\prime} \in \mathcal{S}\left(\mathrm{G}_{\mathrm{S}}^{\prime}\right)$, each distribution on $\mathrm{G}_{\mathbf{A}}^{\prime}$ can be restricted to a linear form on $\mathcal{H}^{\prime S}=\bigotimes_{v \notin \mathrm{S}}^{\prime} \mathcal{H}\left(\mathrm{G}_{v}^{\prime}, \mathrm{K}_{v}^{\prime}\right)$. Using part (3) of Theorem 8.1 the restriction of $\tilde{\mathbf{B}}\left(\sigma^{\prime}, \lambda\right)$ is proportional to $f^{\prime \mathrm{s}} \mapsto \hat{f}^{\prime \mathrm{s}}\left(\mathrm{I}\left(\sigma^{\prime \mathrm{s}}, \lambda\right)\right)$. Therefore, by linear independence of characters we get

Proposition 11.3. - Assume that $\mathrm{E} / \mathrm{F}$ splits at infinity. Then for every $\sigma \in \mathcal{C}^{\mathrm{M}, \tau}, \sigma^{\prime} \in \mathcal{B}(\sigma)$ and $\lambda \in \mathfrak{a}_{\mathrm{M}, \mathbf{G}}^{*}$ we have

$$
\tilde{\mathbf{B}}\left(\sigma^{\prime}, \lambda\right) \longleftrightarrow \mathbf{B}\left(\sigma^{\prime}, \lambda\right)
$$

Eventually, we will remove the assumption on $\mathrm{E} / \mathrm{F}$ in Corollary 12.5 below. 
11.4. Global closed Bessel distributions. - Let $\theta$ be the involution on $\mathrm{G}_{\mathbf{A}}$ defined, as in the local case, by $g^{\theta}=w_{0} \tau\left({ }^{t} g^{-1}\right) w_{0}$ and let $\pi \in \mathcal{C}$. If $\phi$ is a factorizable element of $\pi$ such that $\mathbf{W}^{\pi}(\phi)=\prod_{v} \mathbf{W}_{v}, \mathbf{W}_{v} \in \mathcal{W}^{\psi_{v}}\left(\pi_{v}\right)$ and $\phi^{\theta} \in \hat{\pi}^{\tau}$ is defined by $\phi^{\theta}\left(g^{\theta}\right)=\phi(g)$, $g \in \mathrm{G}_{\mathbf{A}}$ then

(11.14)

$$
\mathbf{W}^{\hat{\pi}, \psi^{-1}}\left(\phi^{\theta}\right)=\prod_{v} \mathfrak{y}_{\pi_{v}}\left(\mathrm{~W}_{v}\right)^{\tau}
$$

Let $\mathcal{C}^{\neg \tau}=\mathcal{C} \backslash \mathcal{C}^{\tau}$. Recall the automorphic induction map ai : $\mathcal{C}^{\mathrm{G}_{n}, \neg \tau} \rightarrow \mathcal{C}^{\mathrm{G}_{2 n}^{\prime}}$ satisfying bc $(\operatorname{aic}(\sigma))=\mathrm{I}\left(\sigma \otimes \sigma^{\tau}, 0\right)$ [AC89].

Let $\kappa=\left(n_{1}, \ldots, n_{s}\right)$ be a composition of $k$ and set $n=2 k, \mathbf{M}=\mathbf{M}_{(\kappa, \overleftarrow{\kappa})}$ and $\mathbf{X}_{\mathbf{M}}=$ $\mathbf{X} \cap w_{0} \mathbf{M}$. Suppose that $\varrho_{i} \in \mathcal{C}^{\mathrm{G}_{n_{i}}, \neg \tau}, i=1, \ldots, s$ and let

$$
\varrho=\varrho_{1} \otimes \cdots \otimes \varrho_{s} \otimes \varrho_{s}^{\tau} \otimes \cdots \otimes \varrho_{1}^{\tau} \in \mathcal{C}^{\mathrm{M}} .
$$

We define $\beta^{\varrho} \in \mathcal{E}_{\mathrm{M}_{\mathbf{A}}}\left(\left(\mathrm{X}_{\mathrm{M}}\right)_{\mathbf{A}}, \varrho^{*}\right)$ by

$$
\beta_{w_{0} \bullet g}^{\varrho}(\phi)=\int_{\left(\mathrm{A}_{\mathrm{M}} \cap \mathrm{M}_{\mathbf{A}}^{w_{0}}\right) \mathrm{M}^{w_{0}} \backslash \mathrm{M}_{\mathbf{A}}^{w_{0}}} \phi(m g) d m, \quad g \in \mathbf{M}_{\mathbf{A}} .
$$

Every element of $\mathbf{M}_{\mathbf{A}}^{w_{0}}$ is of the form $\operatorname{diag}\left(h, h^{\theta}\right)$ where $h \in\left(\mathbf{M}_{\kappa}\right)_{\mathbf{A}}$. If $h=\operatorname{diag}\left(h_{1}, \ldots, h_{s}\right)$ with $h_{i} \in\left(\mathrm{G}_{n_{i}}\right)_{\mathbf{A}}$ then $h^{\theta}=\operatorname{diag}\left(h_{s}^{\theta}, \ldots, h_{1}^{\theta}\right)$. It follows from $(\mathbf{1 1 . 1 4})$ and $(\mathbf{1 0 . 1})$ that we have

$(11.15)$

$$
\beta_{x}^{\varrho}(\phi)=\mathfrak{r} \mathfrak{l}^{\varrho} \prod_{v}\left(\mathfrak{r} \mathfrak{l}_{v}^{\varrho_{v}}\right)^{-1} \beta_{x}^{\varrho_{v}}\left(\mathrm{~W}_{v}\right), \quad x \in\left(\mathrm{X}_{\mathrm{M}}\right)_{\mathbf{A}}
$$

where

$$
\mathbf{W}^{\varrho}(\phi)=\prod_{v} \mathrm{~W}_{v}, \quad \mathrm{~W}_{v} \in \mathcal{W}^{\psi_{v}}\left(\varrho_{v}\right)
$$

For $\lambda \in\left(\mathfrak{a}_{\mathrm{M}}^{*}\right)_{\mathbf{C}}^{w_{0}}$ define $\mathrm{Z}(\varrho, \lambda) \in \mathcal{E}_{\mathrm{G}_{\mathbf{A}}}\left(\mathrm{X}_{\mathbf{A}}, \mathrm{I}_{\mathrm{M}}(\varrho, \lambda)^{*}\right)$ by $\mathrm{Z}(x, \varrho, \lambda)=0$ if $x \bullet \mathrm{G}_{\mathbf{A}} \cap$ $w_{0} \mathrm{M}=\emptyset$ and

$$
\mathrm{Z}(\varphi: x, \varrho, \lambda)=\int_{\mathrm{P}_{\mathbf{A}}^{w_{0}} \backslash \mathrm{G}_{\mathbf{A}}^{w_{0}}} \beta_{w_{0}}^{\varrho}\left(\left.\delta_{\mathrm{P}_{\mathbf{A}}}^{-\frac{1}{2}} \mathrm{I}_{\mathrm{M}}\left(h \iota_{w_{0}}^{x}, \varrho, \lambda\right) \varphi\right|_{\mathrm{M}_{\mathbf{A}}}\right) d h, \quad \varphi \in \mathcal{A}_{\varrho}
$$

if $x=w_{0} \bullet \iota_{w_{0}}^{x}$ for some $\iota_{w_{0}}^{x} \in \mathrm{G}_{\mathbf{A}}$. Note that by $(\mathbf{1 1 . 1 5})$ and $(\mathbf{5 . 6})$ we have

$$
\mathrm{Z}(\varphi: x, \varrho, \lambda)=\mathfrak{r} \mathfrak{l}^{\varrho} \prod_{v}\left(\mathfrak{r} \mathfrak{l}_{v}^{\varrho_{v}}\right)^{-1} \mathrm{Z}\left(\mathrm{W}_{v}: x_{v}, \beta^{\varrho_{v}}, \lambda\right)
$$

where

$$
\mathbf{W}^{\mathrm{M}}(\varphi, \lambda)=\prod_{v} \mathrm{~W}_{v}, \quad \mathrm{~W}_{v} \in \mathrm{I}\left(\mathcal{W}^{\psi_{v}}\left(\varrho_{v}\right)\right)
$$


As before we will obtain a factorization of Bessel distributions. We write $\lambda=$ $\left(\lambda^{\prime}, \overleftarrow{\lambda}^{\prime}\right), \varrho^{\prime}=$ ai $(\varrho)$, i.e., $\varrho^{\prime}=\varrho_{1}^{\prime} \otimes \cdots \otimes \varrho_{s}^{\prime}$ where $\varrho_{i}^{\prime}=$ ai $\left(\varrho_{i}\right)$. Note that

$$
\frac{\mathfrak{r} \mathfrak{l}^{\varrho}}{\mathfrak{c s}\left(\varrho^{\vee},-\lambda\right)}=\mathfrak{r l}^{\prime}\left(\varrho^{\prime}, \lambda^{\prime}\right)^{-1}
$$

and for all $v$

$$
\frac{\mathfrak{r} \varrho_{v}^{\varrho_{v}}}{\mathfrak{c s}_{v}\left(\varrho_{v}^{\vee},-\lambda\right)}=\mathfrak{r l}_{v}^{\prime}\left(\varrho_{v}^{\prime}, \lambda^{\prime}\right)^{-1}
$$

Therefore, the distribution

$$
\tilde{\mathbf{D}}(\varrho, \lambda):=\tilde{\mathfrak{B}}_{\mathfrak{A}(\varrho, \lambda)}^{Z\left((, \lambda), \mathbf{W}_{e}(\hat{\varrho},-\lambda)\right.}
$$

factorizes as

$$
\mathfrak{r} \mathfrak{l}^{\prime}\left(\varrho^{\prime}, \lambda^{\prime}\right)^{-1}\left[\bigotimes_{v} \mathfrak{r} l_{v}^{\prime}\left(\varrho_{v}^{\prime}, \lambda^{\prime}\right) \tilde{\mathbf{D}}\left(\varrho_{v}, \lambda\right)\right]
$$

where $\tilde{\mathbf{D}}\left(\varrho_{v}, \lambda\right)$ is defined in $(\mathbf{5 . 1 6})$. (Evaluating at $\Phi=\bigotimes_{v} \Phi_{v} \in \mathcal{S}\left(\mathrm{X}_{\mathbf{A}}\right)$ almost all the factors are 1.)

Assume now that $x=w_{0} \bullet \iota_{w_{0}}^{x}$. Then in the notation of Appendix $\mathrm{C}$ we have

$$
\alpha_{x}^{(\mathrm{M}, \pi)}(\varphi, \lambda)=\int_{\mathrm{P}_{\mathbf{A}}^{w_{0}} \backslash \mathrm{G}_{\mathbf{A}}^{w_{0}}} \int_{\left(\mathrm{A}_{\mathrm{M}} \cap \mathrm{M}_{\mathbf{A}}^{w_{0}}\right) \mathrm{M}^{w_{0}} \backslash \mathrm{M}_{\mathbf{A}}^{w_{0}}} \varphi_{\lambda}\left(m h \iota_{w_{0}}^{x}\right) d m d h .
$$

It follows that

$$
\alpha_{x}^{(\mathrm{M}, \pi)}(\varphi, \lambda)=\mathrm{Z}(\varphi: x, \varrho, \lambda) .
$$

In other words, we have

$$
\tilde{\mathbf{B}}(\varrho, \lambda)=\tilde{\mathbf{D}}(\varrho, \lambda) .
$$

It follows from Theorem C.3 that

$$
\tilde{\mathbf{D}}(\varrho, \lambda) \longleftrightarrow c_{\kappa} \mathbf{B}\left(\varrho^{\prime}, \lambda^{\prime}\right)
$$

where $c_{\kappa}$ depends only on $\kappa$.

Lemma 11.4. - We have $c_{\kappa}=1$, i.e.,

$$
\tilde{\mathbf{D}}(\varrho, \lambda) \longleftrightarrow \mathbf{B}\left(\varrho^{\prime}, \lambda^{\prime}\right)
$$


Proof. - Choose an everywhere unramified quadratic extension E/F that splits at infinity and at the even places and take $\varrho$ to be everywhere unramified. Let $\mathrm{S}$ be a finite set of places of $\mathrm{F}$, containing the Archimedean places, such that $\psi_{v}^{\prime}$ has conductor $\mathcal{O}_{v}$ for all $v \notin \mathrm{S}$. For every finite place $v \in \mathrm{S}$ let $a_{v} \in \mathrm{F}_{v}^{*}$ be such that $\psi_{v}^{\prime}\left(a_{v}^{-1} \cdot\right)$ has conductor $\mathcal{O}_{v}$. For $v \notin \mathrm{S}$ set $a_{v}=1$. For every finite place $v$ of $\mathrm{F}$ let $f_{v}^{\prime}=\left(\mathbf{1}_{\mathrm{K}_{v}^{\prime}}\right)_{a_{v}}$ and let $\Phi_{v}=\left(\mathbf{1}_{\mathrm{X}_{v} \cap K_{v}}\right)_{a_{v}}$. It follows from Proposition 7.5, Corollary 8.4, (2.14), (9.5), (9.6) and (9.16) together with the factorizations $(\mathbf{1 1 . 3})$ and $(\mathbf{1 1 . 1 6})$ that for every pair of matching functions $\bigotimes_{v \mid \infty} f_{v}^{\prime} \longleftrightarrow \bigotimes_{v \mid \infty} \Phi_{v}$ at the Archimedean places we have

$$
\tilde{\mathbf{D}}\left(\bigotimes_{v} \Phi_{v}: \varrho, \lambda\right)=\mathbf{B}\left(\bigotimes_{v} f_{v}^{\prime}: \varrho^{\prime}, \lambda^{\prime}\right)
$$

Thus $c_{\kappa}=1$.

\section{Local applications}

\section{Local Bessel identities and functional equations-the general case}

In this section we obtain the local Bessel identities introduced in Section 3, i.e., we show that $\mathcal{B I}=\Pi_{\mathrm{bc} \text {-gen }}^{\prime}$. More generally, we show that $\mathcal{B I}_{\mathrm{M}}=\Pi_{\mathrm{bc} \text {-gen }}^{\mathrm{M}^{\prime}}$ for any Levi subgroup M. We also deduce functional equations for the local open periods. The Bessel identities generalize (and refine) the main local results of [LR00] and [Off07] which treat the case $\mathbf{M}^{\prime}=\mathbf{M}_{0}^{\prime}$. New identities are also obtained for the closed periods. The proof is by global means using ideas originated in [LR00] and applying the Bessel identities obtained in Section 7 in the split case, Section 8 for unramified data and Section 11 in the global setting. In order to use the global result we first give a standard globalization argument. Many variants of this are known in the literature. For completeness we provide a proof. In order to simplify notation, for this lemma only $\mathrm{G}=\mathrm{GL}_{n}$ over the number field $\mathrm{F}$.

Lemma 12.1. - Let $\mathrm{S}$ be a finite set of finite places of $\mathrm{F}$ and let $u \notin \mathrm{S}$ be an auxiliary finite place. Let $\mathcal{U}_{\mathrm{S}}$ be a non-empty open subset of $\mathrm{i} \prod_{v \in \mathrm{S}} \mathfrak{a}_{\mathrm{G}}^{*}$ and $\delta_{\mathrm{S}} \in \Pi_{\mathrm{usqr}}^{\mathrm{G}}$. Suppose that there exist two distinct places $u_{1}, u_{2} \in \mathrm{S}$ such that $\delta_{u_{i}} \in \Pi_{\text {cusp }}^{\mathrm{G}_{u_{i}}}, i=1,2$. Then there exists $\pi \in \mathcal{C}$ which is unramified outside $\mathrm{S} \cup\{u\}$ and $\mu \in \mathcal{U}_{\mathrm{S}}$ such that $\pi_{\mathrm{S}} \simeq \delta_{\mathrm{S}}[\mu]$.

Proof. - We first remark that we can assume without loss of generality that $\mathcal{U}_{\mathrm{S}}=$ $\mathrm{i} \prod_{v \in \mathrm{S}} \mathfrak{a}_{\mathrm{G}}^{*}$. Indeed, granted the Lemma in that case, we can always find a unitary Hecke character $\chi: \mathrm{F}^{*} \backslash \mathbf{A}_{\mathrm{F}}^{*} \rightarrow \mathbf{C}^{*}$ which is unramified outside $u$, and such that $\chi_{\mathrm{S}}=e^{\langle\mu, \mathrm{H}(\cdot)\rangle}$ where $\mu$ lies in a prescribed open set of $\mathrm{i} \prod_{v \in \mathrm{S}} \mathfrak{a}_{\mathrm{G}}^{*}$ (cf. [LR00, Corollary 2]).

Henceforth, assume that $\mathcal{U}_{\mathrm{S}}=\mathrm{i} \prod_{v \in \mathrm{S}} \mathfrak{a}_{\mathrm{G}}^{*}$. We apply Arthur's trace formula for $f=$ $\bigotimes_{v} f_{v} \in \mathrm{C}_{c}^{\infty}\left(\mathrm{G}_{\mathbf{A}}\right)$ (or rather, its restriction to Ker $|\mathrm{det}|$ ) where $f_{v}$ are chosen as follows. For $v \in \mathrm{S}$ we take $f_{v}$ to be the product of the characteristic function of Ker $|\operatorname{det}|_{v}$ by 
a pseudo matrix-coefficient of $\delta_{v}$ (cf. [DKV84]). Thus $\operatorname{Tr} \sigma_{v}\left(f_{v}\right)=0$ for any irreducible representation $\sigma_{v}$ of $\mathrm{G}_{v}$ which is either an induced representation from a proper parabolic subgroup or a discrete series representation which is not an unramified twist of $\delta_{v}$. For $v \mid \infty$ we take $f_{v}=g_{v} * \bar{g}_{v} \vee$ where $g_{v} \in \mathrm{C}_{c}^{\infty}\left(\mathrm{G}_{v}\right)$ is any non-zero bi- $\mathrm{K}_{v}$-invariant function. At $u$ we take $f_{u}$ to be the characteristic function of a sufficiently small compact open subgroup of $\mathrm{G}_{u}$. At all other places we take $f_{v}=\mathbf{1}_{\mathrm{K}_{v}}$. By our choice of $f$ and the assumption on $\delta_{\mathrm{S}}$, the spectral side of the trace formula applied to $f$ is $\sum \operatorname{Tr}(\sigma(f))$ where $\sigma$ ranges over the cuspidal representations of $\mathrm{G}_{\mathbf{A}}$ which are unramified outside $\mathrm{S} \cup\{u\}$ and such that $\sigma_{\mathrm{S}}$ is an unramified twist of $\delta_{\mathrm{S}}$. On the geometric side, note that if $f\left(g^{-1} \gamma g\right) \neq 0$ for some $g \in \mathrm{G}_{\mathbf{A}}$ and $\gamma \in \mathrm{G}$ then the coefficients of the characteristic polynomial of $\gamma$ are adelically bounded and $u$-adically close to those of $(t-1)^{n}$ (because of the choice of $f_{u}$ ). Thus $\gamma$ is unipotent. It follows from Arthur's description of the geometric side of the trace formula [Art86] that only the unipotent conjugacy classes contribute. Suppose that $\gamma$ is a nontrivial unipotent element of $\mathrm{G}$. Then there exists a proper parabolic subgroup $\mathrm{P}$ such that the class of $\gamma$ intersects $U$ in a Zariski open dense subset. The invariant unipotent orbital integral of $f_{v}$ at $\gamma$ is given by $\int_{\mathrm{P}_{v} \backslash \mathrm{G}_{v}} \int_{\mathrm{U}_{v}} f_{v}\left(g^{-1} u g\right) d u d g$ [LM09b, Lemma 5.3]. Therefore it vanishes for $v=u_{1}, u_{2}$ since $\delta_{u_{i}}$ is supercuspidal. ${ }^{10}$ Hence, by Arthur's description of the unipotent contribution [Art85], the non-trivial unipotent contributions vanish as well and the geometric expansion reduces to $\operatorname{vol}\left(\mathrm{A}_{\mathrm{G}} \mathrm{G} \backslash \mathrm{G}_{\mathbf{A}}\right) f(e)$. It remains to recall that for all $v \in \mathrm{S}, f_{v}(e)$ is the formal degree of $\delta_{v}$, and therefore $f(e) \neq 0$.

12.1. The main local results. - We turn back to the local inert case. For the next result let $\delta^{\prime} \in \Pi_{\text {sqr }}^{\prime}$ and $\delta=\operatorname{bc}\left(\delta^{\prime}\right)$. Recall that $\delta \in \Pi_{\text {sqr }}$ if and only if $\mathfrak{b}_{\delta}>1$ if and only if $\delta^{\prime} \not \delta^{\prime} \cdot \eta$. In the Archimedean case this is further equivalent to the condition $n=1$.

Proposition 12.2. - Let $\delta^{\prime} \in \Pi_{\mathrm{sqr}}^{\prime}$ and $\delta=\mathrm{bc}\left(\delta^{\prime}\right)$. Then $\delta^{\prime} \in \mathcal{B I}$ and the following conditions are equivalent for any $x \in \mathrm{X}$.

(1) $\delta$ is $\mathrm{G}^{x}$-distinguished.

(2) $\alpha_{x}^{\delta^{\prime}} \not \equiv 0$.

(3) $\mathrm{G}^{x}$ is quasi-split or $\delta \in \Pi_{\mathrm{sqr}}$.

More generally, suppose that $\delta^{\prime} \in \Pi_{\mathrm{sqr}}^{\mathrm{M}^{\prime}}$ and let $\delta=\mathrm{bc}\left(\delta^{\prime}\right)=\delta_{1} \otimes \cdots \otimes \delta_{t}$. Then $\delta^{\prime} \in \mathcal{B} \mathcal{I}^{\mathrm{M}}$ and the following conditions are equivalent for any $x \in \mathrm{X}$.

(1) There exists $\lambda \in \mathfrak{a}_{\mathrm{M}, \mathbf{c}}^{*}$ such that $\mathrm{J}_{\mathrm{M}}\left(x, \alpha^{\delta^{\prime}}, \lambda\right) \not \equiv 0$.

(2) There exists $\lambda \in \mathfrak{a}_{\mathrm{M}, \mathbf{C}}^{*}$ such that $\mathcal{J}_{\delta^{\prime}}\left(x, \alpha^{\delta^{\prime}}, \lambda\right) \not \equiv 0$.

(3) $\mathrm{J}_{\mathrm{M}}\left(x, \alpha^{\delta^{\prime}}, \lambda\right) \not \equiv 0$ whenever holomorphic.

(4) There exists $y \in \mathrm{M} \cap x \bullet \mathrm{G}$ such that $\alpha_{y}^{\delta^{\prime}} \not \equiv 0$.

(5) There exists $y \in \mathrm{M} \cap x \bullet \mathrm{G}$ such that $\delta$ is $\mathrm{M}^{y}$-distinguished.

${ }^{10}$ Actually, this vanishing holds for all $v \in \mathrm{S}$. 
(6) There exists $y=\operatorname{diag}\left(y_{1}, \ldots, y_{t}\right) \in \mathrm{M} \cap x \bullet \mathrm{G}$ such that $\mathrm{G}_{n_{i}}^{y_{i}}$ is quasi-split except possibly (in the p-adic case) for a single index $i_{0}$ for which $\delta_{i_{0}} \in \Pi_{\mathrm{sqr}}$.

(7) $\tilde{\mathfrak{w}}(\delta) \leq \mathfrak{w}(x)$.

(8) In the p-adic case: $\mathrm{G}^{x}$ is quasi-split or $\mathfrak{b}_{\delta}>1$. In the Archimedean case: $\operatorname{dim} \mathfrak{a}_{0}^{\mathrm{M}} \leq \mathfrak{w}(x)$.

Proof. - We begin with the first set of equivalences. Suppose first that $\mathfrak{b}_{\delta}=1$ and $\mathrm{G}^{x}$ is not quasi-split. Then $\delta$ is not $\mathrm{G}^{x}$-distinguished. In the $p$-adic case this follows from Theorem 6.1. In the real case this is because $\mathrm{G}^{x}$ is conjugate to $\mathrm{K}$ and $\delta$ is not unramified. It further follows from the first part of Lemma 3.8 that $\delta^{\prime} \in \mathcal{B} \mathcal{I}_{x}$ (with $\alpha_{x}^{\delta^{\prime}} \equiv 0$ ).

For the first part of the proposition, it remains to see that if $\delta^{\prime} \in \Pi_{\mathrm{sqr}}^{\prime}$ and $\mathrm{G}^{x}$ is quasisplit or $\mathfrak{b}_{\delta}>1$ then $\alpha_{x}^{\delta^{\prime}} \not \equiv 0$ (and $\delta^{\prime} \in \mathcal{B I}_{x}$ ). We may assume without loss of generality that $\delta^{\prime} \in \Pi_{\text {usqr }}^{\prime}$. We use a global argument, starting with the $p$-adic case. Switching the notation, we consider now a quadratic extension of number fields $\mathrm{E} / \mathrm{F}$ which is split at infinity and a place $v_{1}$ of $\mathrm{F}$ such that $\mathrm{E}_{v_{1}} / \mathrm{F}_{v_{1}}$ is our given local extension. Let $y \in \mathrm{X}_{v_{1}}$ and if $\delta^{\prime} \simeq \delta^{\prime} \cdot \eta$ assume further that $\mathrm{G}_{v_{1}}^{y}$ is quasi-split (i.e., $y \in \mathrm{X}_{v_{1}}^{+}$since $n$ is even). Let $v_{2} \neq v_{1}$ be an additional auxiliary finite inert place of F. Fix $\delta_{v_{2}}^{\prime} \in \Pi_{\text {usqr }}^{\mathrm{G}_{v_{2}}^{\prime}}$ such that $\delta_{v_{2}}^{\prime} \nsucc \delta_{v_{2}}^{\prime} \cdot \eta$ (for instance the Steinberg representation). Take $y_{v_{2}} \in \mathrm{X}_{v_{2}}$ such that $\eta_{v_{2}}\left(w_{0} y_{v_{2}}\right)=\eta_{v_{1}}\left(w_{0} y\right)$. Then there exists $x \in \mathrm{X}$ which is contained in the local orbits $y \bullet \mathrm{G}_{v_{1}}, y_{v_{2}} \bullet \mathrm{G}_{v_{2}}$ and $w_{0} \bullet \mathrm{G}_{v}$ for all $v \neq v_{1}, v_{2}$. By Lemma 12.1, there exists $\pi^{\prime} \in \mathcal{C}^{\prime}$ such that $\pi_{v_{1}}^{\prime} \simeq \delta^{\prime}\left[s_{1}\right]$ and $\pi_{v_{2}}^{\prime} \simeq \delta_{v_{2}}^{\prime}\left[s_{2}\right]$ for some $s_{1}, s_{2} \in \mathbf{i R}$. Note that $\pi=\mathrm{bc}\left(\pi^{\prime}\right)$ is cuspidal thanks to our choice of $\delta_{v_{2}}^{\prime}$ and that $\mathrm{G}_{v}^{x}$ is quasi-split for all $v \neq v_{1}, v_{2}$. It follows from part (3) of Corollary 10.3 that $\pi$ is $\mathrm{G}^{x}$-distinguished and that $\pi_{v}^{\prime} \in \mathcal{B I}_{x}$ and $\alpha_{x}^{\pi_{v}^{\prime}} \not \equiv 0$ for all $v$. By $(\mathbf{3 . 1 1})$ and (3.12) we therefore get that $\delta^{\prime} \in \mathcal{B} \mathcal{I}_{y}$ and $\alpha_{y}^{\delta^{\prime}} \not \equiv 0$.

Consider now the case $\mathbf{C} / \mathbf{R}$. In this case $n \leq 2$. The case $n=1$ is trivial and we treat the case $n=2$ by using the quadratic extension $\mathrm{E} / \mathrm{F}=\mathbf{Q}[\sqrt{-1}] / \mathbf{Q}$. Let $y \in \mathrm{X}_{\infty}$. Note that $\delta^{\prime} \simeq \delta^{\prime} \cdot \eta$ for any $\delta^{\prime} \in \Pi_{\text {sqr }}^{\prime}$ and therefore we only need to consider the case $y \in \mathrm{X}_{\infty}^{+}$. Since $n=2$, we have $\mathrm{X}_{\infty}^{+}=w_{0} \bullet \mathrm{G}_{\infty}$ and $\mathrm{X}_{\infty}^{-}=( \pm e) \bullet \mathrm{G}_{\infty}$. Let D be the multiplicative group of the quaternion algebra which ramifies at $\{3, \infty\}$. Take any infinitedimensional irreducible automorphic representation $\sigma$ of $\mathbf{D}_{\mathbf{A}}$ which is trivial at 3 and up to an unramified twist transfers to $\delta^{\prime}$ via the Jacquet-Langlands correspondence at $\infty$. Then the Jacquet-Langlands transfer $\pi^{\prime}$ of $\sigma$ satisfies the following conditions:

(1) $\pi^{\prime} \in \mathcal{C}^{\prime}$,

(2) $\pi_{\infty}^{\prime}$ is an unramified twist of $\delta^{\prime}$,

(3) $\pi_{3}^{\prime}$ is the Steinberg representation.

As before $\pi=\operatorname{bc}\left(\pi^{\prime}\right)$ is cuspidal (since 3 is inert). It follows from the last part of Corollary 10.3 that $\pi$ is $\mathrm{G}^{w_{0}}$-distinguished, $\pi_{\infty}^{\prime} \in \mathcal{B I}_{w_{0}}$ and $\alpha_{w_{0}}^{\pi_{\infty}^{\prime}} \not \equiv 0$. As before, it follows that $\delta^{\prime} \in \mathcal{B I}_{y}$ and $\alpha_{y}^{\delta^{\prime}} \not \equiv 0$. This completes the proof of the first statement.

As for the second statement, the first four conditions are clearly equivalent by part (4) of Proposition 4.1. The other conditions are equivalent by virtue of the first part and the structure of $\mathrm{M} \cap x \bullet \mathrm{G}$. 
Corollary 12.3. - Let $\pi \in \Pi_{\text {gen }}^{\tau}$ and $x \in \mathrm{X}$ be such that $\tilde{\mathfrak{w}}(\pi) \leq \mathfrak{w}(x)$. Then $\pi$ is $\mathrm{G}^{x}-$ distinguished. Thus, Conjecture 6.12 holds for generic representations. In particular, $\pi \in \Pi_{\text {gen }}^{\tau}$ is $\mathrm{G}^{x}$ distinguished if $\mathrm{G}^{x}$ is quasi-split or if $\pi \in \Pi^{\tau, \text { an }}$ (and in particular, by Lemma 3.3 if $\pi \in \Pi_{\mathrm{unr}}$ ).

Proof. - There exists $\delta^{\prime} \in \Pi_{\mathrm{sqr}}^{\mathrm{M}^{\prime}}, \delta=\mathrm{bc}\left(\delta^{\prime}\right)$ such that $\pi=\mathrm{I}(\delta)$. Since $\tilde{\mathfrak{w}}(\pi)=\tilde{\mathfrak{w}}(\delta)$, the corollary follows from the second part of Proposition 12.2 together with Corollary 4.2.

We also remark that in the $p$-adic case, if $\delta \in \Pi_{\mathrm{sqr}}^{\mathrm{M}, \tau}$ and $\Gamma$ is a set of representatives $\left(\right.$ of size $2^{t-1}$ ) for $[\mathcal{B}](\delta)$ then

$$
\left\{\left.\alpha^{\delta^{\prime}}\right|_{x \bullet \mathrm{G} \cap \mathrm{M}}: \delta^{\prime} \in \Gamma\right\} \quad \text { are linearly independent in } \mathcal{E}_{\mathrm{M}}\left(x \bullet \mathrm{G} \cap \mathrm{M}, \delta^{*}\right) .
$$

This follows immediately from the non-vanishing of $\alpha_{x}^{\delta^{\prime}}$ and the relation $(\mathbf{3 . 1 3})$ (for $\mathrm{G}=\mathrm{M}$ ).

We are now ready to state and prove the main local result. Recall the distribution $\tilde{\mathbf{B}}\left(\sigma^{\prime}, \lambda\right)$ defined in $(\mathbf{4 . 1 3})$.

Theorem 12.4. - Let $\mathrm{E} / \mathrm{F}$ be a quadratic extension of local fields. Then for all $\mathrm{M}^{\prime}$ we have $\Pi_{\mathrm{bc}-\text { gen }}^{\mathrm{M}^{\prime}}=\mathcal{B} \mathcal{I}_{\mathrm{M}}$. In particular, $\Pi_{\mathrm{bc}-\text { gen }}^{\prime}=\mathcal{B I}$. Moreover, for any $n$ there exists a sign $v_{n}=v_{n}(\mathrm{E} / \mathrm{F})=$ \pm 1 depending only on $\mathrm{E} / \mathrm{F}$ with $v_{1}=1$ and with the following properties. Fix $\mathrm{M}^{\prime}$ and $\sigma^{\prime} \in \Pi_{\mathrm{bc} \text {-gen }}^{\mathrm{M}^{\prime}}$. Then

(1) As an identity of meromorphic functions in $\lambda \in \mathfrak{a}_{\mathrm{M}, \mathbf{G}}^{*}$ we have

$$
\alpha\left(\sigma^{\prime}, \lambda\right)=v_{\kappa} \mathcal{J}_{\sigma^{\prime}}\left(\alpha^{\sigma^{\prime}}, \lambda\right)
$$

and

$$
\tilde{\mathbf{B}}\left(\sigma^{\prime}, \lambda\right) \longleftrightarrow v_{\kappa} \mathbf{B}\left(\sigma^{\prime}, \lambda\right)
$$

where $v_{\kappa}=\frac{v_{n}}{\prod_{i=1}^{t} v_{n_{i}}}$. In particular, $\tilde{\mathbf{B}}\left(\sigma^{\prime}, \lambda\right)$ is entire.

(2) $\mathcal{J}_{\sigma^{\prime}}\left(\alpha^{\sigma^{\prime}}, \cdot\right)$ is holomorphic at any irreducibility point of $\mathrm{I}\left(\sigma^{\prime}, \lambda\right)$ and we have

$$
\alpha^{\mathrm{I}\left(\sigma^{\prime}, \lambda\right)} \circ \mathbf{W}(\sigma, \lambda)=v_{\kappa} \mathcal{J}_{\sigma^{\prime}}\left(\alpha^{\sigma^{\prime}}, \lambda\right)
$$

(3) If $\mathrm{E} / \mathrm{F}$ is unramified then $v_{n}=1$ for all $n$.

(4) For any $w \in \mathrm{W}(\mathrm{M})$ we have

$$
\mathcal{J}_{w \sigma^{\prime}}\left(\alpha^{w \sigma^{\prime}}, w \lambda\right) \circ \mathrm{N}(w, \mathcal{W}(\sigma), \lambda)=\mathcal{J}_{\sigma^{\prime}}\left(\alpha^{\sigma^{\prime}}, \lambda\right)
$$

Proof. - First note that the relations $(\mathbf{1 2 . 2})$ and $(\mathbf{1 2 . 3})$ are equivalent from the definitions of $\alpha\left(\sigma^{\prime}, \lambda\right), \tilde{\mathbf{B}}\left(\sigma^{\prime}, \lambda\right)$ and $\mathbf{B}\left(\sigma^{\prime}, \lambda\right)$. 
We begin with the $p$-adic case. We first prove that $\Pi_{\mathrm{sqr}}^{\mathrm{M}^{\prime}} \subseteq \mathcal{B I}_{\mathrm{M}}$ and that $(\mathbf{1 2 . 2})$ holds for $\sigma^{\prime}=\delta^{\prime}=\delta_{1}^{\prime} \otimes \cdots \otimes \delta_{t}^{\prime} \in \Pi_{\mathrm{sqr}}^{\mathrm{M}^{\prime}}$ for some sign $v_{\mathrm{M}}$. The relation (12.4) would then follow from Lemma 3.6. By Lemma 3.7, we may assume without loss of generality that $\delta^{\prime} \in \Pi_{\mathrm{usqr}}^{\mathrm{M}^{\prime}}$.

Let $\delta=\operatorname{bc}\left(\delta^{\prime}\right)$. By Proposition 12.2 we have $\delta^{\prime} \in \mathcal{B I}^{\mathrm{M}}$. As in the proof of Proposition 12.2 we switch to global notation. Thus, assume that $\delta^{\prime} \in \Pi_{\text {usqr }}^{\mathrm{M}^{\prime}\left(k^{\prime}\right)}$ and $\delta=\operatorname{bc}\left(\delta^{\prime}\right)$ with respect to a quadratic extension $k / k^{\prime}$ of $p$-adic fields. Choose a quadratic extension of number fields $\tilde{\mathrm{E}} / \tilde{\mathrm{F}}$ which splits at infinity and such that at some place $v_{0}$ of $\tilde{\mathrm{F}}$ we have $\tilde{\mathrm{E}}_{v_{0}} / \tilde{\mathrm{F}}_{v_{0}} \simeq k / k^{\prime}$. We can choose a different quadratic extension $\mathrm{F}$ of $\tilde{\mathrm{F}}$ which splits at $v_{0}$ and such that $\mathrm{F}_{v} \simeq \tilde{\mathrm{E}}_{v}$ at all places $v \neq v_{0}$ of $\tilde{\mathrm{F}}$ which are inert in $\tilde{\mathrm{E}}$ and are even or ramified at $\tilde{\mathrm{E}}$. Let $v_{1}, v_{2}$ be the places of $\mathrm{F}$ above $v_{0}$. Then $\mathrm{E}=\tilde{\mathrm{EF}}$ is a quadratic extension of $\mathrm{F}$ which splits at infinity and such that $\mathrm{E}_{v_{i}} / \mathrm{F}_{v_{i}} \simeq k / k^{\prime}, i=1,2$ and all other inert places of $\mathrm{E} / \mathrm{F}$ are odd and unramified. Let $u, u_{1}, u_{2}$ be three auxiliary finite places of $\mathrm{F}$ which split in $\mathrm{E}$ and let $\mathcal{U} \subseteq\left(\mathrm{ia}_{\mathrm{M}}^{*}\right)^{2}$ be an arbitrary non-empty open set. Denote by $\mathrm{St}^{\mathrm{M}_{v_{2}}^{\prime}}=\mathrm{St}_{n_{1}}^{\prime} \otimes \cdots \otimes \mathrm{St}_{n_{t}}^{\prime}$ the Steinberg representation of the group $\mathrm{M}_{v_{2}}^{\prime}$. It follows from Lemma 12.1 that there exists $\sigma^{\prime}=\sigma_{1}^{\prime} \otimes \cdots \otimes \sigma_{t}^{\prime} \in \mathcal{C}^{\mathrm{M}^{\prime}}$ unramified outside $\left\{v_{1}, v_{2}, u, u_{1}, u_{2}\right\}$ such that $\sigma_{v_{1}}^{\prime} \simeq \delta^{\prime}\left[\mu_{v_{1}}\right]$ and $\sigma_{v_{2}}^{\prime} \simeq \operatorname{St}^{\mathrm{M}_{v_{2}}^{\prime}}\left[\mu_{v_{2}}\right]$ for some $\left(\mu_{v_{1}}, \mu_{v_{2}}\right) \in \mathcal{U}$. In particular, $\sigma_{i}^{\prime} \neq \sigma_{i}^{\prime} \cdot \eta$ for all $i=1, \ldots, t$ since the same holds for the Steinberg representation. Therefore $\sigma=\operatorname{bc}\left(\sigma^{\prime}\right)$ is also cuspidal. Applying Proposition 11.3 and comparing the factorizations $(\mathbf{1 1 . 3})$ and $(\mathbf{1 1 . 1 2})$ we obtain that for every place $v, \sigma_{v}^{\prime} \in \mathcal{B I}_{\mathrm{M}_{v}}$ and there is a non-zero meromorphic function $v_{\mathrm{M}, v}\left(\sigma_{v}^{\prime}, \lambda\right)$ such that

$$
\alpha\left(\sigma_{v}^{\prime}, \lambda\right)=v_{\mathrm{M}, v}\left(\sigma_{v}^{\prime}, \lambda\right) \mathcal{J}_{\sigma_{v}^{\prime}}\left(\alpha^{\sigma_{v}^{\prime}}, \lambda\right)
$$

and moreover $\prod_{v} v_{\mathrm{M}, v}\left(\sigma_{v}^{\prime}, \lambda\right)=1$. Note that by $(\mathbf{9 . 1 0}),(\mathbf{9 . 1 2})$ and $(\mathbf{9 . 8}) v_{\mathrm{M}, v}\left(\sigma_{v}^{\prime}, \lambda\right)$ does not depend on $\psi_{v}^{\prime}$. Taking into account $(\mathbf{7 . 3})$ in the split case and $(\mathbf{8 . 5})$ and Remark 9.3 in the unramified case we infer that

$$
v_{\mathrm{M}, v_{1}}\left(\sigma_{v_{1}}^{\prime}, \lambda\right) v_{\mathrm{M}, v_{2}}\left(\sigma_{v_{2}}^{\prime}, \lambda\right)=1
$$

By Lemma 3.7 and $(\mathbf{4 . 6})$ we have $\delta^{\prime} \in \mathcal{B I}_{\mathrm{M}_{v_{1}}}$ and

$$
v_{\mathrm{M}, v_{1}}\left(\sigma_{v_{1}}^{\prime}, \lambda\right)=v_{\mathrm{M}, v_{1}}\left(\delta^{\prime}, \lambda+\mu_{v_{1}}\right) .
$$

Similarly $\mathrm{St}^{\mathrm{M}_{v_{2}}} \in \mathcal{B I}_{\mathrm{M}_{v_{2}}}$ and

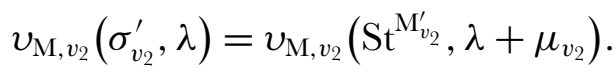

We infer that

$$
v_{\mathrm{M}, v_{1}}\left(\delta^{\prime}, \lambda+\mu_{v_{1}}\right) v_{\mathrm{M}, v_{2}}\left(\mathrm{St}^{\mathrm{M}_{v_{2}}^{\prime}}, \lambda+\mu_{v_{2}}\right)=1 \text {. }
$$


Since $\left(\mu_{v_{1}}, \mu_{v_{2}}\right) \in \mathcal{U}$ and $\mathcal{U}$ was an arbitrary open set we infer that

$$
v_{\mathrm{M}, v_{1}}\left(\delta^{\prime}, \lambda+\mu_{1}\right) v_{\mathrm{M}, v_{2}}\left(\mathrm{St}^{\mathrm{M}_{v_{2}}^{\prime}}, \lambda+\mu_{2}\right)=1
$$

for all $\mu_{1}, \mu_{2}$. Hence $v_{\mathrm{M}, v_{1}}=v_{\mathrm{M}, v_{1}}\left(\delta^{\prime}, \lambda\right)$ is independent of both $\delta^{\prime}$ and $\lambda$. Moreover, taking $\delta^{\prime}=\mathrm{St}^{\mathrm{M}_{v_{2}}}$, we infer that $v_{\mathrm{M}, v_{1}}=v_{\mathrm{M}, v_{2}}$ is a sign depending only on $\mathrm{M}$ and the local quadratic extension. Switching back to the local notation, we simply denote it by $v_{\mathrm{M}}$. This shows (12.2) in the square-integrable case up to the determination of the sign.

Consider the general case of $\sigma^{\prime} \in \Pi_{\mathrm{bc} \text {-gen }}^{\mathrm{M}^{\prime}}$. Let $\mathrm{L}^{\prime}$ be a Levi subgroup of $\mathrm{M}^{\prime}, \delta^{\prime} \in \Pi_{\mathrm{sqr}}^{\mathrm{L}^{\prime}}$ and $\mu \in \mathfrak{a}_{\mathrm{L}, \mathbf{G}}^{*}$ be such that $\sigma^{\prime} \simeq \mathrm{I}_{\mathrm{L}^{\prime}}^{\mathrm{M}^{\prime}}\left(\delta^{\prime}, \mu\right)$. Applying the above argument for $\delta^{\prime}$ with respect to both $\mathrm{G}^{\prime}$ and $\mathrm{M}^{\prime}$ and using Lemmas 4.4 and 3.6 we obtain

$$
v_{\mathrm{L}}^{\mathrm{M}} \alpha\left(\sigma^{\prime}, \lambda\right)=v_{\mathrm{L}} \mathcal{J}_{\sigma^{\prime}}\left(\alpha^{\sigma^{\prime}}, \lambda\right) .
$$

In particular, (12.2) holds whenever $\sigma^{\prime}$ (resp. $\sigma$ ) is a principal series representation of $\mathbf{M}^{\prime}$ (resp. $\mathbf{M}$ ) with proportionality constant $v_{\mathrm{M}_{0}} / v_{\mathrm{M}_{0}}^{\mathrm{M}}$. Using Lemma 12.1, and a global setting as before, this time for $\mathrm{S}=\left\{v_{1}, u_{1}, u_{2}\right\}$, we immediately obtain that $v_{\mathrm{M}}=v_{\mathrm{M}_{0}} / v_{\mathrm{M}_{0}}^{\mathrm{M}}$. Thus $v_{\mathrm{M}}=\frac{v_{n}}{\prod_{i} v_{n_{i}}}$ where we set $v_{n}=v_{\mathrm{M}_{0}}^{\mathrm{G}}$. Hence $v_{\mathrm{M}}=v_{\kappa}$, and it also follows that $v_{\mathrm{L}} v_{\mathrm{L}}^{\mathrm{M}}=v_{\mathrm{M}}$. We obtain $(\mathbf{1 2 . 2})$ from $(\mathbf{1 2 . 6})$.

For $\mathrm{E} / \mathrm{F}$ unramified it now follows from $(\mathbf{8 . 5})$ that $v_{n}=1$.

We turn to the Archimedean case. Consider first the case where $\delta^{\prime} \in \Pi_{\text {usqr }}^{\mathrm{M}^{\prime}}$. Take $\mathrm{E} / \mathrm{F}=\mathbf{Q}(\sqrt{-1}) / \mathbf{Q}$. Choose a globalization $\sigma^{\prime}=\sigma_{1}^{\prime} \otimes \cdots \otimes \sigma_{t}^{\prime}$ of $\delta^{\prime}$ such that at the prime 2 (denoted by the place $v_{2}$ ) we have $\left(\sigma_{i}^{\prime}\right)_{v_{2}} \not 4\left(\sigma_{i}^{\prime}\right)_{v_{2}} \cdot \eta_{v_{2}}$ for all $i$ and set $\sigma=$ $\operatorname{bc}\left(\sigma^{\prime}\right)$. (For existence of such $\sigma^{\prime}$ we may argue as in the Archimedean part of the proof of Proposition 12.2.) It follows from Lemma 2.2 and strong multiplicity one that the distributions $\bigotimes_{p<\infty} \mathfrak{r l} \mathfrak{l}^{\prime}\left(\sigma_{p}^{\prime \prime}, \lambda\right) \mathbf{B}\left(\sigma_{p}^{\prime \prime}, \lambda\right), \sigma^{\prime \prime} \in \mathcal{B}(\sigma)$ are linearly independent. It further follows from the result already established in the $p$-adic case that

$$
\bigotimes_{p<\infty} \mathfrak{r} \mathfrak{l}^{\prime}\left(\sigma_{p}^{\prime \prime}, \lambda\right) \tilde{\mathbf{B}}\left(\sigma_{p}^{\prime \prime}, \lambda\right) \longleftrightarrow v_{\mathrm{M}, v_{2}} \bigotimes_{p<\infty} \mathfrak{r} \mathfrak{l}^{\prime}\left(\sigma_{p}^{\prime \prime}, \lambda\right) \mathbf{B}\left(\sigma_{p}^{\prime \prime}, \lambda\right)
$$

The factorizations $(\mathbf{1 1 . 3})$ and $(\mathbf{1 1 . 1 2})$ together with the global identity $(\mathbf{1 1 . 1 3})$ imply that

$$
\tilde{\mathbf{B}}\left(\delta^{\prime}, \lambda\right) \longleftrightarrow v_{\mathrm{M}, v_{2}} \mathbf{B}\left(\delta^{\prime}, \lambda\right)
$$

In particular, $v_{\mathrm{M}, v_{\infty}}=v_{\mathrm{M}, v_{2}}$ is independent of $\delta^{\prime}$ and $\lambda$. Back in a local Archimedean setting, arguing as before in the $p$-adic case, one now proves the identities (12.2) for any $\sigma^{\prime} \in \Pi_{\mathrm{bc}-\text { gen }}^{\mathrm{M}^{\prime}}$ with $v_{n, v_{\infty}}=v_{n, v_{2}}$.

Finally, we show that $(\mathbf{1 2 . 5})$ follows from $(\mathbf{1 2 . 4})$. Indeed, since $\pi^{\prime}:=\mathrm{I}\left(\sigma^{\prime}, \lambda\right) \simeq$ $\mathrm{I}\left(w \sigma^{\prime}, w \lambda\right)$ and $v_{w \kappa}=v_{\kappa}$, applying $(\mathbf{1 2 . 2})$ to $\left(w \sigma^{\prime}, w \mathrm{M} w^{-1}\right)$ we get

$$
\alpha^{\pi^{\prime}} \circ \mathbf{W}(w \sigma, w \lambda)=v_{\kappa} \mathcal{J}_{w \sigma^{\prime}}\left(\alpha^{w \sigma^{\prime}}, w \lambda\right)
$$


Composing this with $\mathrm{N}(w, \mathcal{W}(\sigma), \lambda)$ and using the functional equation $(\mathbf{1 . 1 0})$ we obtain

$$
\alpha^{\pi^{\prime}} \circ \mathbf{W}(\sigma, \lambda)=v_{\kappa} \mathcal{J}_{w \sigma^{\prime}}\left(\alpha^{w \sigma^{\prime}}, w \lambda\right) \circ \mathrm{N}(w, \mathcal{W}(\sigma), \lambda)
$$

Using (12.4) once again we infer (12.5).

From $(\mathbf{1 1 . 1 3})$ we now deduce

Corollary 12.5. - Proposition 11.3 holds for any quadratic extension of numbers fields $\mathrm{E} / \mathrm{F}$ (not necessarily split at infinity). Moreover, $\prod_{v} v_{n}\left(\mathrm{E}_{v} / \mathrm{F}_{v}\right)=1$.

Remark 12.6. - In order to determine the signs $v_{n}$ it is necessary to have more information about the matching at the ramified places. For instance, a factor $\eta(-1)$ cannot be discerned at the split or unramified places, and can be incorporated into the transfer factor at will, resulting in a possible sign change in the $\alpha$ 's.

12.2. Bessel identities for closed Bessel distributions. - Next, we prove a similar relation for the closed periods. Recall the distribution $\tilde{\mathbf{D}}_{\mathrm{L}}(\varrho, \lambda)$ defined in $(\mathbf{5 . 1 6})$.

Proposition 12.7. - For any $k \geq 0$ and a local extension of quadratic fields $\mathrm{E} / \mathrm{F}$ there exist signs $\varsigma_{k}$ with the following property. Let $n=2 k, \kappa$ a composition of $k, \mathrm{~L}=\mathrm{M}_{(\kappa, \overleftarrow{\kappa})}$ an even symmetric Levi and $\varrho=\sigma \otimes \overleftarrow{\sigma^{\tau}} \in \Pi_{\text {gen }}^{\mathrm{L}, w_{0}}$. Then for any $\lambda=(\mu, \overleftarrow{\mu}) \in\left(\mathfrak{a}_{\mathrm{L}}^{*}\right)_{\mathbf{G}}^{w_{0}}$ such that $\mathrm{I}(\varrho, \lambda)$ is irreducible we have

$$
\alpha^{\pi^{\prime}} \circ \mathbf{W}(\varrho, \lambda)=\varsigma_{k} \lambda_{\psi^{\prime}}^{k} \mathrm{Z}\left(\beta^{\varrho}, \lambda\right)
$$

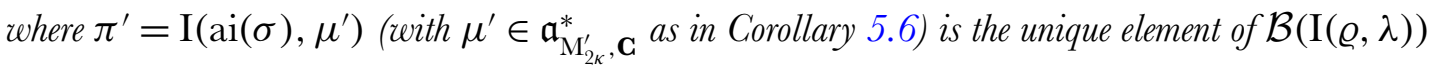
such that $\pi^{\prime} \cdot \eta=\pi^{\prime}$. Equivalently,

$(\mathbf{1 2 . 8})$

$$
\tilde{\mathbf{D}}(\varrho, \lambda) \longleftrightarrow \varsigma_{k} \mathbf{B}_{\mathrm{I}\left(\mathrm{ai}(\sigma), \mu^{\prime}\right)}
$$

Proof. - The equivalence of $(\mathbf{1}$ 2.7) and $(\mathbf{1 2 . 8})$ follows from the definition $(\mathbf{5 . 1 6})$ of $\tilde{\mathbf{D}}$ and the relation $(\mathbf{2 . 9})$ applied to the equivalence

$$
\left(\mathbf{W}(\varrho, \lambda), \mathbf{W}\left(\varrho^{\vee},-\lambda\right)\right): \mathrm{I}(\mathfrak{W}(\varrho), \lambda) \rightarrow \mathfrak{W}(\mathrm{I}(\varrho, \lambda)) .
$$

By Corollary 5.10 and $(\mathbf{2 . 1 4})$ we reduce $(\mathbf{1 2 . 8})$ to the case where $\sigma=\delta_{1} \otimes \cdots \otimes \delta_{s} \in$ $\Pi_{\text {usqr }}^{\mathrm{M}_{\kappa}}$. In this case we argue exactly as in the proof of Theorem 12.4. First note that by (2.14), (9.5), (9.6) and $(\mathbf{9 . 1 6})$ the identity $(\mathbf{1 2 . 8})$ is independent of $\psi^{\prime}$. In the $p$-adic case, switching to a global (split at infinity) setting, we globalize $\delta_{i}$ to $\varrho_{i} \in \mathcal{C}^{\mathrm{G}_{n_{i}}, \neg \tau}, i=1, \ldots, s$ (by using an auxiliary split place). By (11.17) we get (12.8) up to a constant. The argument in the proof of Theorem 12.4 using Proposition 7.5 and Corollary 8.4 shows that the proportionality constant is a sign which depends only on $k$. For the real case we argue once again as in Theorem 12.4 applying $(\mathbf{1 1 . 1 7})$ to $\mathbf{Q}[\sqrt{-1}] / \mathbf{Q}$. 
Now let $\mathrm{L}=\mathrm{M}_{(\kappa, m, \overleftarrow{\kappa})}$ be odd symmetric. We will use the setup of Proposition 5.4 and its notation. Namely, let $w=w_{(m, k, k)}^{(m+k, k)}, \tilde{\mathrm{L}}=w^{-1} \mathrm{~L} w=\mathrm{M}_{(m, \kappa, \overleftarrow{\kappa})}, \tilde{\mathrm{M}}=\mathrm{M}_{(m, 2 k)}, \widetilde{w_{*}}=$ $\operatorname{diag}\left(1_{m}, w_{0}^{\mathrm{G}_{2 k}}\right)$.

Let $\varrho=\sigma \otimes \delta \otimes \overleftarrow{\delta}^{\tau} \in \Pi_{\text {gen }}^{\tilde{\mathrm{L}}, \widetilde{w_{*}}}$ with $\sigma \in \Pi_{\text {gen }}^{\mathrm{G}_{m}, \tau}$ and $\delta \in \Pi_{\text {gen }}^{\mathrm{M}_{\kappa}}$. For any $\alpha \in$ $\mathcal{E}_{\mathrm{G}_{m}}\left(\mathrm{X}_{m}, \mathcal{W}(\sigma)^{*}\right)$ and $\left.\beta \in \mathcal{E}_{\mathrm{M}_{(\kappa, \overleftarrow{\kappa})}}\left(\mathrm{X}_{\mathrm{M}_{(\kappa, \overleftarrow{k})}^{\mathrm{G}_{2 k}}, \mathcal{W}(\delta \otimes \overleftarrow{\delta} \tau}\right)^{*}\right)$ define $\alpha \otimes \beta \in \mathcal{E}_{\tilde{\mathrm{L}}}\left(\mathrm{X}_{\tilde{\mathrm{L}}}^{\tilde{\mathrm{M}}}, \mathcal{W}(\varrho)^{*}\right)$ by

$$
(\alpha \otimes \beta)_{\operatorname{diag}\left(x_{1}, x_{2}\right)}=\alpha_{x_{1}} \otimes \beta_{x_{2}}, \quad x_{1} \in \mathrm{X}_{m}, \quad x_{2} \in \mathrm{X}_{\mathrm{M}_{(\kappa, \overleftarrow{\kappa})}^{\mathrm{G}_{2 k}}}
$$

We will view $w(\alpha \otimes \beta)$ as an element of $\mathcal{E}_{\mathrm{L}}\left(\mathrm{X}_{\mathrm{L}}^{\mathrm{M}_{(k, m, k)}}, \mathcal{W}(w \varrho)^{*}\right)$ by identifying $w \mathcal{W}(\varrho)$ with $\mathcal{W}(w \varrho)$ (as in our convention for the intertwining operators $\mathrm{N}(w, \mathcal{W}(\varrho), \lambda)$ ).

Proposition 12.8. - With the notation above, for any $\lambda=(z, \mu, \overleftarrow{\mu}) \in\left(\mathfrak{a}_{\tilde{\mathrm{L}}}^{*}\right)_{\mathbf{G}}^{\tilde{w}}$ with $z \in \mathbf{G}$, $\mu \in \mathfrak{a}_{\mathrm{M}_{\kappa}, \mathbf{G}}^{*}$ such that $\mathrm{I}(\varrho, \lambda) \in \Pi_{\text {gen }}$ and for any $\sigma^{\prime} \in \mathcal{B}(\sigma)$ we have

$$
\alpha^{\mathrm{I}\left(\varrho^{\prime}, 0\right)} \circ \mathbf{W}(w \varrho, w \lambda)=\frac{v_{m+2 k}}{v_{m} v_{2 k}} \varsigma_{k} \lambda_{\psi^{\prime-1}}^{(1-m) k} \mathrm{Z}\left(w\left(\alpha^{\sigma^{\prime}} \otimes \beta^{\delta \otimes \overleftarrow{\delta} \tau}\right), w \lambda\right)
$$

as elements of $\mathcal{E}_{\mathrm{G}}\left(\mathrm{X}, \mathrm{I}(\mathcal{W}(w \varrho), w \lambda)^{*}\right)$ where $\varrho^{\prime}=\sigma^{\prime}[z] \otimes \mathrm{I}\left(\mathrm{ai}(\delta), \mu^{\prime}\right) \in \Pi_{\text {gen }}^{\tilde{\mathrm{M}}^{\prime}}$.

Proof. - First note that by $(\mathbf{1 . 1 0})$ and $(\mathbf{1 . 6})$ we have

$$
\begin{aligned}
\mathbf{W}(w \varrho, w \lambda)= & \mathbf{W}(\varrho, \lambda) \circ \mathrm{N}(w, \mathcal{W}(\varrho), \lambda)^{-1} \\
= & \mathbf{W}_{\tilde{\mathrm{M}}}\left(\mathrm{I}_{\tilde{\mathrm{L}}}^{\tilde{\mathrm{M}}}(\varrho, \lambda), 0\right) \circ \mathrm{I}_{\tilde{\mathrm{M}}}\left(\mathbf{W}_{\tilde{\mathrm{L}}}^{\tilde{\mathrm{M}}}(\varrho, \lambda)\right) \\
& \circ \Gamma_{\tilde{\mathrm{L}}, \tilde{\mathrm{M}}}(\mathcal{W}(\varrho), \lambda) \circ \mathrm{N}(w, \mathcal{W}(\varrho), \lambda)^{-1} .
\end{aligned}
$$

Recall that $\operatorname{bc}\left(\varrho^{\prime}\right)=\mathrm{I}(\varrho, \lambda)=\mathrm{I}_{\tilde{\mathrm{M}}}\left(\mathrm{I}_{\tilde{\mathrm{L}}}^{\tilde{\mathrm{M}}}(\varrho, \lambda), 0\right)$. Thus, by $(\mathbf{1 2 . 4})$, the left-hand side of $(\mathbf{1 2 . 9})$ is

$$
\frac{v_{m+2 k}}{v_{m} v_{2 k}} \mathcal{J}_{\varrho^{\prime}}\left(\alpha^{\varrho^{\prime}}, 0\right) \circ \mathrm{I}_{\tilde{\mathrm{M}}}\left(\mathbf{W}_{\tilde{\mathrm{L}}}^{\tilde{\mathrm{M}}}(\varrho, \lambda)\right) \circ \Gamma_{\tilde{\mathrm{L}}, \tilde{\mathrm{M}}}(\mathcal{W}(\varrho), \lambda) \circ \mathrm{N}(w, \mathcal{W}(\varrho), \lambda)^{-1}
$$

Note that $\alpha^{\varrho^{\prime}}=\alpha^{\sigma^{\prime}[z]} \otimes \alpha^{\mathrm{I}\left(\mathrm{ai}(\delta), \mu^{\prime}\right)}$. Therefore we infer from $(\mathbf{1 2 . 7})$ that

$$
\mathcal{J}_{\varrho^{\prime}}\left(\alpha^{\varrho^{\prime}}, 0\right) \circ \mathrm{I}_{\tilde{\mathrm{M}}}\left(\mathbf{W}_{\tilde{\mathrm{L}}}^{\tilde{\mathrm{M}}}(\varrho, \lambda)\right)=\varsigma_{k} \boldsymbol{\lambda}_{\psi^{\prime}}^{k} \mathcal{J}_{\varrho^{\prime}}\left(\mathrm{Z}^{\tilde{\mathrm{M}}}\left(\alpha^{\sigma^{\prime}} \otimes \beta^{\delta \otimes \overleftarrow{\delta}^{\tau}}, \lambda\right), 0\right)
$$

The proposition therefore follows from $(\mathbf{5 . 1 4})$ since $\boldsymbol{\lambda}_{\psi^{\prime-1}}=\eta(-1) \boldsymbol{\lambda}_{\psi^{\prime}}$. tion $(\mathbf{3 . 8}))$.

The following Corollary strengthens Corollary 12.3 (taking into account the rela-

Corollary 12.9. - Let $\pi^{\prime} \in \Pi_{\mathrm{bc}-\text { gen }}^{\prime}$. Then $\alpha_{x}^{\pi^{\prime}} \not \equiv 0$ iff $\tilde{\mathfrak{w}}^{\prime}\left(\pi^{\prime}\right) \leq \mathfrak{w}(x)$. In particular, in the p-adic case $\alpha_{x}^{\pi^{\prime}} \not \equiv 0$ unless $\mathrm{G}^{x}$ is not quasi-split and $\pi^{\prime} \simeq \pi^{\prime} \cdot \eta$. 
Proof. - The $p$-adic case follows from Lemma 3.8 (and will also follow from the argument below).

By Lemma 3.2 we can write $\pi^{\prime}=\sigma^{\prime} \times$ ai $(\varrho)$ where $\varrho \in \Pi_{\text {gen }}^{\mathrm{G}_{k}}, k=\tilde{\mathfrak{w}}^{\prime}\left(\pi^{\prime}\right)$ and $\tilde{\mathfrak{w}}^{\prime}\left(\sigma^{\prime}\right)=0$. By $(\mathbf{1 2 . 9})$ the non-vanishing of $\alpha_{x}^{\pi^{\prime}}$ is equivalent to the non-vanishing of $\mathrm{Z}\left(x, w\left(\alpha^{\sigma^{\prime}} \otimes \beta^{\varrho \otimes} \overleftarrow{\varrho}^{\tau}\right), 0\right)$. In view of Lemma 5.2 and Lemma 5.1 part (1), it suffices to prove the Corollary for $\sigma^{\prime}$. That is, we may assume that $\tilde{\mathfrak{w}}^{\prime}\left(\pi^{\prime}\right)=0$.

Suppose therefore that $\tilde{\mathfrak{w}}^{\prime}\left(\pi^{\prime}\right)=0$. We have to show that $\alpha_{x}^{\pi^{\prime}} \not \equiv 0$ for all $x$. We reduce the statement further to the tempered case. Write $\pi^{\prime}$ as $\pi^{\prime}=\mathrm{I}\left(\sigma^{\prime}, \lambda\right)$ where $\sigma^{\prime} \in \Pi_{\text {temp }}^{\mathrm{M}^{\prime}}$ and $\lambda=\left(\lambda_{1}, \ldots, \lambda_{t}\right) \in\left(\mathfrak{a}_{\mathrm{M}}^{*}\right)_{+}$. By Theorem 12.4 the non-vanishing of $\alpha_{x}^{\pi^{\prime}}$ is equivalent to that of $\mathcal{J}_{\sigma^{\prime}}\left(x, \alpha^{\sigma^{\prime}}, \lambda\right)$. Recall that $\mathrm{C}_{\mathrm{M}}\left(w_{\mathrm{M}}: \sigma, \cdot ; \psi\right)$ is holomorphic and non-zero at $\lambda(\mathbf{1 . 1 1})$ and therefore the same is true for $\mathrm{C}_{\mathrm{M}^{\prime}}\left(w_{\mathrm{M}}: \sigma^{\prime}, \lambda ; \psi^{\prime}\right)$. Hence, the non-vanishing of $\mathcal{J}_{\sigma^{\prime}}\left(x, \alpha^{\sigma^{\prime}}, \lambda\right)$ is equivalent to that of $\mathrm{J}\left(x, \alpha^{\sigma^{\prime}}, \lambda\right)$. In turn, the latter condition is equivalent to the existence of $y \in \mathrm{M} \cap x \bullet \mathrm{G}$ such that $\alpha_{y}^{\sigma^{\prime}} \not \equiv 0$. Therefore it suffices to prove the corollary for $\sigma^{\prime}$.

Hence, we can assume that $\pi^{\prime} \in \Pi_{\text {temp }}^{\prime}$ and $\tilde{\mathfrak{w}}^{\prime}\left(\pi^{\prime}\right)=0$. Thus $\pi^{\prime}$ is induced from $\delta^{\prime}=\delta_{1}^{\prime} \otimes \cdots \otimes \delta_{t}^{\prime} \in \Pi_{\mathrm{usqr}}^{\mathrm{M}^{\prime}}$ with $\delta_{i}^{\prime} \not \neq \delta_{j}^{\prime} \cdot \eta$ for any $i, j$. Let $\delta=\operatorname{bc}\left(\delta^{\prime}\right) \in \Pi_{\mathrm{usqr}}^{\mathrm{M}}$. It follows from Lemma 1.1 that $\mathfrak{n}_{\mathrm{M}^{\prime}}\left(\delta^{\prime}, \lambda\right)$ is holomorphic and non-zero at $\lambda=0$. By the same argument as before, the non-vanishing of $\alpha_{x}^{\pi^{\prime}}$ follows from the analogous statement in the square-integrable case. The latter follows from Proposition 12.2.

Remark 12.10. - The Corollary gives an alternative proof of a result of Jacquet on the non-vanishing of $\alpha_{x}^{\pi^{\prime}}$ in the quasi-split case [Jac10].

Combining Corollaries 10.3 and 12.9 we obtain Theorem 0.1.

12.3. An example. - We finish this section with the following example which shows that in general, local distinction does not imply global distinction. Let $\tilde{\mathrm{E}} / \mathrm{F}$ be a quadratic extension of number fields with Galois involution $\tilde{\tau}$ and corresponding quadratic character $\tilde{\eta}: \mathrm{F}^{*} \backslash \mathbf{A}_{\mathrm{F}}^{*} \rightarrow\{ \pm 1\}$. Let $\tilde{\mathrm{G}}_{n}=\operatorname{Res}_{\tilde{\mathrm{E}} / \mathrm{F}} \mathrm{GL}_{n}$. Suppose that $\sigma \in \mathcal{C}^{\tilde{\mathrm{G}}_{n}, \neg \tilde{\tau}}$ and let $\pi^{\prime}=\operatorname{ai}_{\tilde{\mathrm{E}} / \mathrm{F}}(\sigma) \in \mathcal{C}^{\mathrm{G}_{2 n}^{\prime}}$ so that $\pi^{\prime}=\pi^{\prime} \cdot \tilde{\eta}$. Let $\mathrm{E} / \mathrm{F}$ be another quadratic extension such that $\pi^{\prime} \neq \pi^{\prime} \cdot \eta$. (For instance, this is the case if $\mathrm{E}_{v} / \mathrm{F}_{v}$ is ramified at a place where $\pi_{v}^{\prime}$ is unramified.) Thus, $\pi=\mathrm{bc}\left(\pi^{\prime}\right) \in \mathcal{C}^{\mathrm{G}_{2 n}}$. Let $x \in \mathrm{X}_{2 n}$ and let $\mathrm{S}_{x}$ be the (finite) set of places of $\mathrm{F}$ which are inert in $\mathrm{E}$ and such that $x$ is not quasi-split with respect to $\mathrm{E}_{v} / \mathrm{F}_{v}$. Assume that for any $v \in \mathrm{S}_{x} \pi_{v}$ is unramified and $\tilde{\mathrm{E}}_{v} / \mathrm{F}_{v} \simeq \mathrm{E}_{v} / \mathrm{F}_{v}$. Then by Corollary $12.3 \pi$ is locally $\mathrm{G}^{x}\left(\mathrm{~F}_{v}\right)$-distinguished for all $v$. On the other hand, by Corollary 10.3 $\pi$ is not globally $\mathrm{G}^{x}$-distinguished unless $x$ is quasi-split with respect to $\mathrm{E} / \mathrm{F}$ (i.e., unless $\left.\mathrm{S}_{x}=\emptyset\right)$. For otherwise, if $v \in \mathrm{S}_{x}$ then $\pi_{v}^{\prime}=\pi_{v}^{\prime} \cdot \eta_{v}$ since $\tilde{\eta}_{v}=\eta_{v}$ and hence $\alpha_{x}^{\pi_{v}^{\prime}} \equiv 0$ by Corollary 12.9. There is of course no difficulty in choosing $x$ which is not quasi-split and satisfying the properties above since $\tilde{\mathrm{E}}_{v} / \mathrm{F}_{v} \simeq \mathrm{E}_{v} / \mathrm{F}_{v}$ for infinitely many inert places $v$ of $\mathrm{F}$. 


\section{Multiplicity of unitary periods}

In this section we study the space of local unitary periods. Let $\mathrm{E} / \mathrm{F}$ be a quadratic extension of $p$-adic fields. For simplicity of notation, in the following we will identify all generic irreducible representations with their Whittaker models.

13.1. The supercuspidal case. - The following Theorem of Jacquet describes the space of local unitary periods in the supercuspidal case. It was proved in [Jac01, §5] for the case $n=3$ and $x=e$. Recall the notation $\mathcal{B}(\pi)$ and $[\mathcal{B}](\pi)$ from Section 3.2.

Theorem 13.1 facquet. - Let $\pi \in \Pi_{\text {cusp. }}^{\tau}$. Then for any $x \in \mathrm{X}$ we have $\operatorname{dim} \operatorname{Hom}_{\mathrm{G}^{x}}(\pi, \mathbf{C})=1$ and moreover, there exists $v^{\vee} \in \pi^{\vee}$ such that

$$
v \mapsto \int_{\mathrm{G}^{x}} v^{\vee}(\pi(g) v) d g
$$

is a non-zero element in $\operatorname{Hom}_{\mathrm{G}^{x}}(\pi, \mathbf{G})$.

Proof. - Since $\mathcal{B}(\pi)=\left\{\pi^{\prime}, \pi^{\prime} \cdot \eta\right\}$ for some $\pi^{\prime} \not \pi^{\prime} \cdot \eta$ in $\Pi_{\text {cusp }}^{\prime}$, it follows from Proposition 12.2 that $\pi$ is $\mathrm{G}^{x}$-distinguished (and in fact $\left.\alpha_{x}^{\pi^{\prime}} \not \equiv 0\right)$. Without loss of generality we may further assume that $\pi$ is unitary. The rest of the proof follows [Jac01, §5] verbatim.

Corollary 13.2. - Let $\sigma=\sigma_{1} \otimes \cdots \otimes \sigma_{t} \in \Pi_{\text {cusp }}^{\mathrm{M}, \tau}$ with $\sigma_{1}, \ldots, \sigma_{t}$ distinct. Then for every $\sigma^{\prime} \in \mathcal{B}(\sigma)$ and $x \in \mathrm{X}$, the linear form $\mathrm{J}\left(x, \alpha^{\sigma^{\prime}}, \cdot\right)$ on $\mathrm{I}(\sigma)$ is holomorphic at 0 . Furthermore, if $\Gamma$ is a set of representatives (of size $\left.2^{t-1}\right)$ for $[\mathcal{B}](\sigma)$ then the set $\left\{\mathrm{J}\left(x, \alpha^{\sigma^{\prime}}, 0\right): \sigma^{\prime} \in \Gamma\right\}$ is a basis of $\operatorname{Hom}_{\mathrm{G}^{x}}(\mathrm{I}(\sigma), \mathbf{G})$.

Proof. - Recall that by $(\mathbf{1 2 . 1})$ the set $\left\{\left.\alpha^{\sigma^{\prime}}\right|_{x \bullet \mathrm{G} \cap \mathrm{M}}: \sigma^{\prime} \in \Gamma\right\}$ is linearly independent in $\mathcal{E}_{\mathrm{M}}\left(x \bullet \mathrm{G} \cap \mathrm{M}, \sigma^{*}\right)$. On the other hand, by Lemma 6.10 , unitary periods on $\mathrm{I}(\sigma)$ are supported on open orbits. It therefore follows from Theorem 13.1 that $\left\{\left.\alpha^{\sigma^{\prime}}\right|_{x \bullet \mathrm{G} \cap \mathrm{M}}: \sigma^{\prime} \in\right.$ $\Gamma\}$ is a basis for $\mathcal{E}_{\mathrm{M}}\left(x \bullet \mathrm{G} \cap \mathrm{M}, \sigma^{*}\right)$. The lemma now follows from Lemma 6.7.

13.2. The square-integrable case. — Next we study the essentially square-integrable case.

Proposition 13.3. - For any $\delta \in \Pi_{\mathrm{sqr}}$ and $x \in \mathrm{X}$ we have $\operatorname{dim} \operatorname{Hom}_{\mathrm{G}^{x}}(\delta, \mathbf{G}) \leq 1$.

Proof. - Suppose that $\delta=\Delta_{\varrho}([a, b])$ for $\varrho \in \Pi_{\text {cusp }}^{\mathrm{G}_{d}}$ and let $\mathrm{P}=\mathrm{MU}$ be the parabolic subgroup of type $(d, \ldots, d)(t=b-a+1$ times $)$. If $\varrho \notin \Pi^{\tau}$ then $\delta \notin \Pi^{\tau}$. Hence, by Theorem 6.1 we may assume that $\varrho=\operatorname{bc}\left(\varrho^{\prime}\right)$ for some $\varrho^{\prime} \not \varrho^{\prime} \cdot \eta \in \Pi_{\text {cusp }}^{\mathrm{G}_{d}^{\prime}}$, in which case $\delta=\operatorname{bc}\left(\delta^{\prime}\right)$ where $\delta^{\prime}=\Delta_{\varrho^{\prime}}^{\prime}([a, b])$. Set $\sigma=\varrho[a] \otimes \cdots \otimes \varrho[b]$ and $\sigma^{\prime}=\varrho^{\prime}[a] \otimes \cdots \otimes \varrho^{\prime}[b]$ so that $\delta$ is the unique irreducible quotient of $\mathrm{I}(\sigma)$. Let $p: \mathrm{I}(\sigma) \rightarrow \delta$ be the projection. 
For $k=1, \ldots, t-1$ let $w_{k} \in \mathrm{W}(\mathrm{M})$ be the permutation associated with the simple reflection $(k, k+1)$ when identifying $\mathrm{W}(\mathrm{M})$ with the symmetric group on $[1, t]$. The operator $\mathrm{M}\left(w_{k}, w_{k} \sigma, w_{k} \lambda\right)$ is holomorphic at $\lambda=0$ and the image of $\mathrm{M}\left(w_{k}, w_{k} \sigma, 0\right)$ is a proper submodule of $\mathrm{I}(\sigma)$. Let

$$
\mathcal{V}:=\sum_{k=1}^{t-1} \operatorname{Im} \mathrm{M}\left(w_{k}, w_{k} \sigma, 0\right) \subseteq \operatorname{Ker} p .
$$

(In fact, it is known that equality holds but we do not need to know this a priori.)

It follows from $(\mathbf{1 2 . 5})$ that for every $k=1, \ldots, t-1$ and $v=\left(v_{1}, \ldots, v_{t}\right) \in \Xi^{t}$ we have the functional equation

$$
\begin{aligned}
& \mathcal{J}_{\sigma^{\prime} \cdot v}\left(x, \alpha^{\sigma^{\prime} \cdot v}, \lambda\right) \circ \mathrm{M}\left(w_{k}, w_{k} \sigma, w_{k} \lambda\right) \\
& \quad=\mathrm{C}_{\mathrm{M}}^{\mathrm{G}}\left(w_{k}: w_{k} \sigma, w_{k} \lambda ; \psi\right)^{-1} \mathcal{J}_{w_{k}\left(\sigma^{\prime} \cdot v\right)}\left(x, \alpha^{w_{k}\left(\sigma^{\prime} \cdot v\right)}, w_{k} \lambda\right)
\end{aligned}
$$

as rational functions in $q_{\mathrm{F}}^{\lambda}$. In terms of the unnormalized functionals this relation becomes

$$
\begin{aligned}
& \mathrm{J}\left(x, \alpha^{\sigma^{\prime} \cdot v}, \lambda\right) \circ \mathrm{M}\left(w_{k}, w_{k} \sigma, w_{k} \lambda\right) \\
& =c \gamma\left(\lambda_{k+1}-\lambda_{k}+1, \varrho^{\prime} \times \varrho^{\prime \vee} \cdot v_{k+1} v_{k}^{-1} ; \psi^{\prime}\right)^{-1} \\
& \quad \times \gamma\left(\lambda_{k}-\lambda_{k+1}-1, \varrho^{\prime} \times \varrho^{\prime \vee} \cdot v_{k} v_{k+1}^{-1} \eta ; \psi^{\prime}\right)^{-1} \mathrm{~J}\left(x, \alpha^{w_{k}\left(\sigma^{\prime} \cdot v\right)}, w_{k} \lambda\right)
\end{aligned}
$$

where $c \neq 0$ is a constant. Since $\varrho^{\prime} \nsucceq \varrho^{\prime} \cdot \eta$, it follows from Lemma 1.1 that the product

$$
\begin{aligned}
& \gamma\left(\lambda_{k+1}-\lambda_{k}+1, \varrho^{\prime} \times \varrho^{\prime \vee} \cdot v_{k+1} v_{k}^{-1} ; \psi^{\prime}\right)^{-1} \\
& \quad \times \gamma\left(\lambda_{k}-\lambda_{k+1}-1, \varrho^{\prime} \times \varrho^{\prime \vee} \cdot v_{k} v_{k+1}^{-1} \eta ; \psi^{\prime}\right)^{-1}
\end{aligned}
$$

is holomorphic at $\lambda=0$ and it is non-zero there if and only if $v_{k} \neq v_{k+1}$. Recall that by Corollary $13.2, \mathrm{~J}\left(x, \alpha^{\sigma^{\prime} \cdot \nu}, \lambda\right)$ is holomorphic at $\lambda=0$. Thus, for any $v \in \Xi^{t}$ there is a scalar $c_{k}(v) \in \mathbf{C}$ such that

$$
\mathrm{J}\left(x, \alpha^{\sigma^{\prime} \cdot v}, 0\right) \circ \mathrm{M}\left(w_{k}, w_{k} \sigma, 0\right)=c_{k}(v) \mathrm{J}\left(x, \alpha^{w_{k}\left(\sigma^{\prime} \cdot v\right)}, 0\right)
$$

and

$$
c_{k}(v) \neq 0 \quad \text { if and only if } \quad v_{k} \neq v_{k+1} .
$$

(Note that for $v=1$ this implies that $\mathrm{J}\left(x, \alpha^{\sigma^{\prime}}, 0\right)$ factors through $\mathrm{I}(\sigma) / \mathcal{V}$, which as was pointed out before, is in fact isomorphic to $\delta$.)

We will prove the Proposition by showing that for any $\ell \in \operatorname{Hom}_{\mathrm{G}^{x}}(\delta, \mathbf{G}), \ell \circ p \in$ $\operatorname{Hom}_{\mathrm{G}^{x}}(\mathrm{I}(\sigma), \mathbf{C})$ is proportional to $\mathrm{J}\left(x, \alpha^{\sigma^{\prime}}, 0\right)$. 
Let $\ell \in \operatorname{Hom}_{\mathrm{G}^{x}}(\delta, \mathbf{C})$. By Corollary 13.2 there exist complex numbers $\left\{a_{v}\right\}_{v \in \Xi^{t}: \nu_{1}=1}$ such that

$$
\ell \circ p=\sum_{\nu \in \Xi^{t}: v_{1}=1} a_{\nu} \mathrm{J}\left(x, \alpha^{\sigma^{\prime} \cdot \nu}, 0\right)
$$

Since $\ell \circ p$ is trivial on $\mathcal{V}$, for any $k=1, \ldots, t-1$ we have

$$
\sum_{\nu \in \Xi^{\prime}: \nu_{1}=1} a_{\nu} \mathrm{J}\left(x, \alpha^{\sigma^{\prime} \cdot v}, 0\right) \circ \mathrm{M}\left(w_{k}, w_{k} \sigma, 0\right) \equiv 0 .
$$

Together with $(\mathbf{1 3 . 1})$ we get

$$
\sum_{\nu \in \Xi^{t}: v_{1}=1} a_{v} c_{k}(v) \mathrm{J}\left(x, \alpha^{w_{k}\left(\sigma^{\prime} \cdot \nu\right)}, 0\right) \equiv 0 .
$$

By (13.2) and Corollary 13.2 (applied to $w_{k} \sigma^{\prime}$ ) we conclude that $a_{v}=0$ for all $v \in \boldsymbol{\Xi}^{t}$ such that $v_{1}=1$ and $v_{k} \neq v_{k+1}$. Applying this to all $k$, we infer that $\ell \circ p$ is proportional to $\mathrm{J}\left(x, \alpha^{\sigma^{\prime}}, 0\right)$ as required.

Remark 13.4. - In its notation, the proof shows that $\mathrm{J}\left(x, \alpha^{\sigma^{\prime}}, 0\right)$ factors through $\delta$ as a non-zero multiple of $\alpha_{x}^{\delta^{\prime}}$ (since the latter is non-zero). The same is true for $\mathcal{J}_{\sigma^{\prime}}\left(x, \alpha^{\sigma^{\prime}}, 0\right)$ by $(\mathbf{4 . 5})$ and Lemma 1.1 .

It is also possible to generalize the second part of Theorem 13.1 to the squareintegrable case. However, we will not discuss this aspect here.

From Theorem 6.1 and Propositions 13.3 and 12.2 together with the fact that $\mathfrak{b}_{\delta}=2$ for $\delta \in \Pi_{\mathrm{sqr}}^{\tau}$ we infer

Corollary 13.5. - For any $\delta \in \Pi_{\mathrm{sqr}}$ and $x \in \mathrm{X}$ we have

$$
\operatorname{dim} \operatorname{Hom}_{\mathrm{G}^{x}}(\delta, \mathbf{C})= \begin{cases}1 & \delta \in \Pi_{\mathrm{sqr}}^{\tau}, \\ 0 & \text { otherwise. }\end{cases}
$$

As in Corollary 13.2 we now get

Corollary 13.6. - Let $\delta=\delta_{1} \otimes \cdots \otimes \delta_{t} \in \Pi_{\mathrm{sqr}}^{\mathrm{M}, \tau}$ with no $i<j$ such that $\delta_{i} \unlhd \delta_{j}$. Then for every $\delta^{\prime} \in \mathcal{B}(\delta)$ and $x \in \mathrm{X}$ the linear form $\mathrm{J}\left(x, \alpha^{\delta^{\prime}}\right.$, $)$ on $\mathrm{I}(\delta)$ is holomorphic at 0 . Furthermore, if $\Gamma$ is a set of representatives (of size $\left.2^{t-1}\right)$ for $[\mathcal{B}](\delta)$ then the set $\left\{\mathrm{J}\left(x, \alpha^{\delta^{\prime}}, 0\right): \delta^{\prime} \in \Gamma\right\}$ is a basis of $\operatorname{Hom}_{\mathrm{G}^{x}}(\mathrm{I}(\delta), \mathbf{C})$.

We also have the following property. 
Lemma 13.7. - Let $\delta \in \Pi_{\mathrm{usqr}}^{\mathrm{M}, \tau}$ and $\delta^{\prime} \in \mathcal{B}(\delta)$. Then $\mathcal{J}_{\delta^{\prime}}\left(\alpha^{\delta^{\prime}}, \lambda\right)$ is holomorphic for $\operatorname{Re} \lambda \in$ $-\left(\mathfrak{a}_{\mathrm{M}}^{*}\right)_{+} \cdot$

Proof. - By Theorem 12.4, $\tilde{\mathbf{B}}\left(\delta^{\prime}, \lambda\right)$ is entire. Suppose that $\operatorname{Re} \lambda \in-\left(\mathfrak{a}_{\mathrm{M}}^{*}\right)_{+}$. Then by [JS83] $\mathbf{W}_{e}\left(\delta^{\vee},-\lambda\right) \in \mathrm{I}\left(\delta^{\vee},-\lambda\right)^{*}$ is injective. Hence, the Lemma follows from $(\mathbf{4 . 1 3})$, Lemma 2.3 and (2.11).

Definition 13.8. - Let $\pi \in \Pi$. Assume that $\pi$ is the unique irreducible quotient of $\mathrm{I}(\delta, \lambda)$ where $\delta=\delta_{1} \otimes \cdots \otimes \delta_{t} \in \Pi_{\mathrm{usqr}}^{\mathrm{M}}$ and $\lambda \in \overline{\left(\mathfrak{a}_{\mathrm{M}}^{*}\right)_{+}}$. We say that $\pi$ has regular parameters if $\delta_{1}\left[\lambda_{1}\right], \ldots, \delta_{t}\left[\lambda_{t}\right]$ are pairwise non-isomorphic. We write

$$
\Pi_{\text {reg }}^{\tau}=\left\{\pi_{1} \times \pi_{2}: \pi_{1} \in \Pi^{\tau, \mathrm{ti}}, \pi_{2} \in \Pi^{\tau, \text { an }}, \pi_{2} \text { with regular parameters }\right\} .
$$

Proposition 13.9. - For any $\pi \in \Pi_{\mathrm{reg}}^{\tau}$ and $x \in \mathrm{X}$ we have $\operatorname{dim} \operatorname{Hom}_{\mathrm{G}^{x}}(\pi, \mathbf{G}) \leq[\mathfrak{b}]_{\pi}$. (Recall that $[\mathfrak{b}]_{\pi}=\frac{1}{2} \mathfrak{b}_{\pi}$ as long as $\pi \notin \Pi^{\tau, \mathrm{ti}}$.)

Proof. - By $(\mathbf{6 . 9})$ and the fact that $\mathfrak{b}_{\pi}=\mathfrak{b}_{\pi_{2}}$ we reduce to the case where $\pi \in \Pi^{\tau \text {,an }}$. In this case $\pi$ is the Langlands quotient of $\mathrm{I}(\delta, \lambda)$ with $\delta=\delta_{1} \otimes \cdots \otimes \delta_{t} \in \Pi_{\text {usqr }}^{\mathrm{M}, \tau}$ and $\lambda=\left(\lambda_{1}, \ldots, \lambda_{t}\right) \in \overline{\left(\mathfrak{a}_{\mathrm{M}}^{*}\right)_{+}}$. By the regularity condition on $\pi$ we have $\delta_{i}\left[\lambda_{i}\right] \not \varnothing \delta_{j}\left[\lambda_{j}\right]$ for all $i \neq j$. Hence, the Proposition follows from Corollary 13.6 applied to $\delta[\lambda]$ together with the injectivity of the natural map $\operatorname{Hom}_{\mathrm{G}^{x}}(\pi, \mathbf{C}) \rightarrow \operatorname{Hom}_{\mathrm{G}^{x}}(\mathrm{I}(\delta, \lambda), \mathbf{G})$.

13.3. Ladder representations. - We can extend the uniqueness result of Proposition 13.3 to a wider class of representations.

Definition 13.10. - Let $\sigma \in \Pi_{\text {cusp }}^{\mathrm{G}_{d}}$ and let $a_{1}>\cdots>a_{t}, b_{1}>\cdots>b_{t}$ be integers such that $a_{i} \leq b_{i}$ for $i=1, \ldots, t$ and $b_{i+1} \geq a_{i}-1, i=1, \ldots, t-1$. Let $\Delta_{i}=\Delta_{\sigma}\left(\left[a_{i}, b_{i}\right]\right)$. (Thus, $\Delta_{i} \times \Delta_{i+1}$ is reducible for all $i=1, \ldots, t-1$.) The Langlands quotient $\pi$ of $\Delta_{1} \times \cdots \times \Delta_{t}$ will be called a (proper) ladder representation ${ }^{11}$ and will be denoted by $\mathrm{L}\left(\Delta_{1}, \ldots, \Delta_{t}\right)$.

Important special cases of ladder representations are the Speh representations $u(\delta, t)$ for any $\delta \in \Pi_{\text {usqr }}$ and $t \geq 1$ which comprise the building blocks for the unitary dual of $\mathrm{GL}_{n}$ [Tad86]. By definition $u(\delta, t)$ is the Langlands quotient of $\delta[(t-1) / 2] \times$ $\cdots \times \delta[(1-t) / 2]$. The class of ladder representations is closed under Zelevinsky involution. This follows from the combinatorial description of the latter [MW86].

Theorem 13.11. - For any ladder representation $\pi=\mathrm{L}\left(\Delta_{1}, \ldots, \Delta_{t}\right)$ and $x \in \mathrm{X}$ we have

$$
\operatorname{dim} \operatorname{Hom}_{\mathrm{G}^{x}}(\pi, \mathbf{C}) \leq 1
$$

and equality holds if and only if $\pi \in \Pi^{\tau}$.

\footnotetext{
${ }^{11}$ There is an unrelated, much earlier notion of ladder representations for unitary groups.
} 
Proof. - The proof of the first part is similar to the proof of Proposition 13.3. Note that $\pi^{\tau}=\mathrm{L}\left(\Delta_{1}^{\tau}, \ldots, \Delta_{t}^{\tau}\right)$ and therefore by uniqueness of Langlands data, if $\pi \in \Pi^{\tau}$ then $\Delta_{i} \in \Pi^{\tau}$ and therefore $\sigma \in \Pi^{\tau}$. Thus, we can assume that $\sigma=\operatorname{bc}\left(\sigma^{\prime}\right)$ for some $\sigma^{\prime} \in \Pi^{\prime}$ with $\sigma^{\prime} \not \sigma^{\prime} \cdot \eta$.

Let $\delta=\Delta_{1} \otimes \cdots \otimes \Delta_{t} \in \Pi^{\mathrm{M}}$ where $\mathrm{P}=\mathrm{MU}$ is the corresponding parabolic. Thus, $\pi$ is the Langlands quotient of $\mathrm{I}(\delta)$. Let $w_{k} \in \mathrm{W}(\mathrm{M}), k=1, \ldots, t-1$ be as in the proof of Proposition 13.3 and let

$$
\mathcal{V}:=\sum_{k=1}^{t-1} \operatorname{Ker} \mathrm{M}\left(w_{k}, \delta, 0\right)=\sum_{k=1}^{t-1} \operatorname{Im} \mathrm{N}\left(w_{k}^{-1}, w_{k} \delta, 0\right) \subseteq \operatorname{KerM}\left(w_{\mathrm{M}}, \delta, 0\right)
$$

where the second equality (and the holomorphy of $\mathrm{N}\left(w_{k}^{-1}, w_{k} \delta, w_{k} \lambda\right)$ at 0$)$ follow from Lemma 1.1 . We recall that the projection $p: \mathrm{I}(\delta) \rightarrow \pi$ is essentially the intertwining operator $\mathrm{M}\left(w_{\mathrm{M}}, \delta, 0\right)$, so that $\operatorname{Ker} \mathrm{M}\left(w_{\mathrm{M}}, \delta, 0\right)$ is the unique maximal submodule of $\mathrm{I}(\delta)$. Let

$$
\delta^{\prime}=\Delta_{\sigma^{\prime}}^{\prime}\left(\left[a_{1}, b_{1}\right]\right) \otimes \cdots \otimes \Delta_{\sigma^{\prime}}^{\prime}\left(\left[a_{t}, b_{t}\right]\right)
$$

It follows from $(\mathbf{1 2 . 5})$ that for every $k=1, \ldots, t-1$ and $v=\left(v_{1}, \ldots, v_{t}\right) \in \Xi^{t}$ we have the functional equations

$$
\mathrm{J}\left(x, \alpha^{\delta^{\prime} \cdot v}, \lambda\right) \circ \mathrm{N}\left(w_{k}^{-1}, w_{k} \delta, w_{k} \lambda\right)=c c_{k}(\nu, \lambda) \mathrm{J}\left(x, \alpha^{w_{k}\left(\delta^{\prime} \cdot v\right)}, w_{k} \lambda\right)
$$

where $c \neq 0$ is a constant and

$$
c_{k}(v, \lambda)=\frac{\gamma\left(\lambda_{k+1}-\lambda_{k}, \delta_{k}^{\prime \vee} \times \delta_{k+1}^{\prime} \cdot \eta v_{k} v_{k+1}^{-1} ; \psi^{\prime}\right)}{\gamma\left(\lambda_{k}-\lambda_{k+1}, \delta_{k}^{\prime} \times \delta_{k+1}^{\prime} \cdot \eta v_{k} v_{k+1}^{-1} ; \psi^{\prime}\right)}
$$

It follows from Lemma 1.1 and the assumption on $\pi$ that $c_{k}(\nu, \lambda)$ is holomorphic at $\lambda=0$, and writing $c_{k}(\nu)=c_{k}(\nu, 0)$ we have

$$
c_{k}(v) \neq 0 \quad \text { if and only if } \quad v_{k}=v_{k+1} .
$$

Let $\ell \in \operatorname{Hom}_{\mathrm{G}^{x}}(\pi, \mathbf{C})$. By Corollary 13.6, there exist complex numbers $\left\{a_{\nu}\right\}_{\nu \in \Xi^{t}: \nu_{1}=1}$ such that

$$
\ell \circ p=\sum_{\nu \in \Xi^{t}: \nu_{1}=1} a_{\nu} \mathrm{J}\left(x, \alpha^{\delta^{\prime} \cdot v}, 0\right) .
$$

Since $\ell \circ p$ vanishes on $\mathcal{V}$, we have

$$
\sum_{\nu \in \Xi^{t}: \nu_{1}=1} a_{\nu} \mathrm{J}\left(x, \alpha^{\delta^{\prime} \cdot \nu}, 0\right) \circ \mathrm{N}\left(w_{k}^{-1}, w_{k} \delta, 0\right) \equiv 0, \quad k=1, \ldots, t-1 .
$$


Using the functional equations $(\mathbf{1 3 . 3})$ we get

$$
\left.\sum_{\nu \in \Xi^{t}: \nu_{1}=1} a_{\nu} c_{k}(v) J\left(x, \alpha^{w_{k}\left(\delta^{\prime} \cdot v\right)}, w_{k} \lambda\right)\right|_{\lambda=0} \equiv 0 .
$$

Recall that by $(\mathbf{1 2 . 1})$ the set $\left\{\left.\alpha^{w_{k}\left(\delta^{\prime} \cdot v\right)}\right|_{x \bullet \mathrm{G} \cap \mathrm{M}}: v \in \boldsymbol{\Xi}^{t}, v_{1}=1\right\}$ is linearly independent in $\mathcal{E}_{\mathrm{M}}\left(x \bullet \mathrm{G} \cap \mathrm{M}, \delta^{*}\right)$. Let $\mathrm{J}^{\circ}\left(x, \alpha^{w_{k}\left(\delta^{\prime} \cdot v\right)}, w_{k} \lambda\right)$ be the restriction of $\mathrm{J}\left(x, \alpha^{w_{k}\left(\delta^{\prime} \cdot v\right)}, w_{k} \lambda\right)$ to sections supported on $\mathrm{G}^{\circ}[x]=\left\{g \in \mathrm{G}: x \bullet g^{-1} \in \mathrm{X}^{\circ}\right\}$ (the open orbits). Recall that $\mathrm{J}^{\circ}\left(x, \alpha^{w_{k}\left(\delta^{\prime} \cdot v\right)}, w_{k} \lambda\right)$ is holomorphic in $\lambda$ and by $(\mathbf{6 . 6})$ the set $\left\{\mathrm{J}^{\circ}\left(x, \alpha^{w_{k}\left(\delta^{\prime} \cdot v\right)}, 0\right): v \in \mathbf{\Xi}^{t}\right.$, $\left.v_{1}=1\right\}$ is linearly independent. Also,

$$
\sum_{\nu \in \Xi^{t}: \nu_{1}=1} a_{\nu} c_{k}(\nu) \mathrm{J}^{\circ}\left(x, \alpha^{w_{k}\left(\delta^{\prime} \cdot v\right)}, 0\right) \equiv 0 .
$$

We therefore get by $(\mathbf{1 3 . 4})$ that $a_{v}=0$ whenever $v_{k}=v_{k+1}$. Since this holds for all $k$ we infer that $\ell \circ p$ is proportional to $\mathrm{J}\left(x, \alpha^{\delta^{\prime} \cdot v_{\mathrm{M}}}, 0\right)$ where $v_{\mathrm{M}}$ is as in $(\mathbf{4 . 1})$. The first part follows.

To prove the second part, we use the fact proved in [LM] that $\mathcal{V}$ is in fact equal to the kernel of $\mathrm{M}\left(w_{\mathrm{M}}, \delta, 0\right)$. Therefore, it suffices to show that $\mathrm{J}\left(x, \alpha^{\delta^{\prime} \cdot v_{\mathrm{M}}}, 0\right)$ vanishes on $\mathcal{V}$, i.e., that $\mathrm{J}\left(x, \alpha^{\delta^{\prime} \cdot \nu_{\mathrm{M}}}, 0\right) \circ \mathrm{N}\left(w_{k}^{-1}, w_{k} \delta, 0\right)=0$ for all $k$. We will write the right-hand side of (13.3) for $v=v_{\mathrm{M}}$ differently as

$$
\begin{aligned}
& \gamma\left(\lambda_{k}-\lambda_{k+1}, \delta_{k}^{\prime} \times \delta_{k+1}^{\prime}{ }^{\vee} ; \psi^{\prime}\right)^{-1} \\
& \quad \times \mathrm{J}_{\mathrm{M}_{k}}\left(x, \mathcal{J}_{\mathrm{L}, w_{k}\left(\delta^{\prime} \cdot v_{\mathrm{M}}\right)}^{\mathrm{M}_{k}}\left(\alpha^{w_{k}\left(\delta^{\prime} \cdot v_{\mathrm{M}}\right)},\left(w_{k} \lambda\right)_{\mathrm{L}}^{\mathrm{M}_{k}}\right),\left(w_{k} \lambda\right)_{\mathrm{M}_{k}}\right)
\end{aligned}
$$

where $\mathrm{M}_{k}$ is the Levi of type $\left(n_{1}, \ldots, n_{k-1}, n_{k}+n_{k+1}, n_{k+2}, \ldots, n_{t}\right)$ and $\mathrm{L}=w_{k} \mathrm{M} w_{k}^{-1}$. By Lemma 1.1 and the assumption on $\pi$ we have $\gamma\left(0, \delta_{k}^{\prime} \times \delta_{k+1}^{\prime}{ }^{\vee} ; \psi^{\prime}\right)=\infty$. On the other hand, by Lemma 13.7

$$
\mathcal{J}_{\mathrm{L}, w_{k}\left(\delta^{\prime} \cdot v_{\mathrm{M}}\right)}^{\mathrm{M}_{k}}\left(\alpha^{w_{k}\left(\delta^{\prime} \cdot v_{\mathrm{M}}\right)},\left(w_{k} \lambda\right)_{\mathrm{L}}^{\mathrm{M}_{k}}\right)
$$

is holomorphic at $\lambda=0$ and we can apply Lemma 6.11 and Lemma 6.7 (with respect to $\mathrm{M}_{k}$ and $\left.\delta_{1} \otimes \cdots \otimes \delta_{k-1} \otimes \delta_{k} \times \delta_{k+1} \otimes \delta_{k+2} \otimes \cdots \otimes \delta_{t}\right)$ to infer that

$$
\mathrm{J}_{\mathrm{M}_{k}}\left(x, \mathcal{J}_{\mathrm{L}, w_{k}\left(\delta^{\prime} \cdot v_{\mathrm{M}}\right)}^{\mathrm{M}_{k}}\left(\alpha^{w_{k}\left(\delta^{\prime} \cdot v_{\mathrm{M}}\right)},\left(w_{k} \lambda\right)_{\mathrm{L}}^{\mathrm{M}_{k}}\right),\left(w_{k} \lambda\right)_{\mathrm{M}_{k}}\right)
$$

is holomorphic at $\lambda=0$. Our claim follows.

Corollary 13.12. - Conjecture 6.12 holds for any irreducible representation induced from ladder representations. In particular, it holds for any unitarizable representation.

Proof. - We already know the necessity part of the Conjecture (in general). The sufficiency part follows from the previous Theorem using Corollary 4.2. The last part follows from the description of the unitary dual of G [Tad86]. 
Remark 13.13. - In the complex case, every unitarizable representation is parabolically induced from a character. Therefore, Conjecture 6.12 holds for unitarizable representations in the Archimedean case as well.

13.4. Generic representations.

Proposition 13.14. - Let $\pi \in \Pi_{\text {gen }}^{\tau}$. Then the elements $\left\{\alpha^{\pi^{\prime}}: \pi^{\prime} \in \mathcal{B}(\pi)\right\}$ of $\mathcal{E}_{\mathrm{G}}\left(\mathrm{X}, \pi^{*}\right)$ are linearly independent and hence $\operatorname{dim} \mathcal{E}_{\mathrm{G}}\left(\mathrm{X}, \pi^{*}\right) \geq \mathfrak{b}_{\pi}$. Moreover, for any $x \in \mathrm{X}$ and a set $\Gamma$ of representatives for $[\mathcal{B}](\pi)$, the non-zero elements in

$$
\left\{\alpha_{x}^{\pi^{\prime}}: \pi^{\prime} \in \Gamma\right\}
$$

are linearly independent in $\operatorname{Hom}_{\mathrm{G}^{x}}(\pi, \mathbf{G})$. Thus $\operatorname{dim} \operatorname{Hom}_{\mathrm{G}^{x}}(\pi, \mathbf{G}) \geq[\mathfrak{b}]_{\pi}-\delta_{(x, \pi)}$ where

$$
\delta_{(x, \pi)}= \begin{cases}1 & \text { if } \mathrm{G}^{x} \text { is not quasi-split and } \pi=\delta \times \delta^{\tau} \text { for some } \delta \in \Pi_{\mathrm{gen}}, \\ 0 & \text { otherwise. }\end{cases}
$$

Proof. - By Lemma 2.2 the distributions $\left\{\mathbf{B}_{\pi^{\prime}}: \pi^{\prime} \in \mathcal{B}(\pi)\right\}$ are linearly independent. By Theorem 12.4 and smooth transfer, we infer that $\tilde{\mathfrak{B}}_{\mathfrak{W}^{\psi}(\pi)}^{\alpha^{\pi^{\prime}}, \delta_{e}^{\pi^{\vee}}}, \pi^{\prime} \in \mathcal{B}(\pi)$ are also linearly independent. Hence, the linear independence of $\left\{\alpha^{\pi^{\prime}}: \pi^{\prime} \in \mathcal{B}(\pi)\right\}$ follows from (2.12).

To prove the second part, let $\epsilon=\eta\left(x w_{0}\right)$ so that $x \in \mathrm{X}^{\epsilon}$ and let

$$
[\mathcal{B}]_{\epsilon}(\pi)= \begin{cases}\left\{\left[\pi^{\prime}\right] \in[\mathcal{B}](\pi): \pi^{\prime} \not \pi^{\prime} \cdot \eta\right\} & \epsilon=-1, \\ {[\mathcal{B}](\pi)} & \epsilon=1 .\end{cases}
$$

Choose a set of representatives $\Gamma_{\epsilon}$ for $[\mathcal{B}]_{\epsilon}(\pi)$. Since there is at most one $\pi^{\prime} \in \mathcal{B}(\pi)$ such that $\pi^{\prime} \simeq \pi^{\prime} \cdot \eta$, we have $\left|\Gamma_{\epsilon}\right|=[\mathfrak{b}]_{\pi}-\delta_{(x, \pi)}$.

By Lemma 3.8 and Theorem 12.4, $\alpha_{x}^{\pi^{\prime}} \not \equiv 0$ if and only if $\left[\pi^{\prime}\right] \in \mathcal{B}_{\epsilon}(\pi)$.

We claim that the restrictions of the distributions $\mathbf{B}_{\pi^{\prime}}, \pi^{\prime} \in \Gamma_{\epsilon}$ to $\mathcal{S}\left(\mathrm{G}^{\prime \epsilon}\right)$ are linearly independent. Indeed, otherwise there would exist a non-trivial linear combination

$$
\sum_{\pi^{\prime} \in \Gamma_{\epsilon}} a_{\pi^{\prime}} \mathbf{B}_{\pi^{\prime}}\left(f^{\prime}+\epsilon f_{\eta}^{\prime}\right)=0 \quad \forall f^{\prime} \in \mathcal{S}\left(\mathrm{G}^{\prime}\right) .
$$

By $(2.15)$ it follows that

$$
\sum_{\pi^{\prime} \in \Gamma_{\epsilon}} a_{\pi^{\prime}} \mathbf{B}_{\pi^{\prime}}+\sum_{\pi^{\prime} \in \Gamma_{\epsilon}} \epsilon a_{\pi^{\prime}} \mathbf{B}_{\pi^{\prime} \cdot \eta}=0
$$

This contradicts the linear independence of $\mathbf{B}_{\pi^{\prime}}, \pi^{\prime} \in \mathcal{B}(\pi)$.

As before, using in addition the property (3.5) we infer (using smooth transfer) that the distributions $\mathfrak{B}_{\mathfrak{W}^{\psi}(\pi)}^{\alpha_{x}^{\pi^{\prime}}, \delta_{e}^{\pi^{\vee}}}, \pi^{\prime} \in \Gamma_{\epsilon}$ on $\mathrm{G}$, and hence the functionals $\left\{\alpha_{x}^{\pi^{\prime}}: \pi^{\prime} \in \Gamma_{\epsilon}\right\}$, are linearly independent. The Proposition follows. 
Corollary 13.15. - Let $\pi \in \Pi_{\text {gen,reg }}^{\tau}$ and $x \in \mathrm{X}$. Then

$$
\operatorname{dim} \operatorname{Hom}_{\mathrm{G}^{x}}(\pi, \mathbf{C})= \begin{cases}0 & \text { if } \mathfrak{b}_{\pi}=1 \text { and } x \in \mathrm{X}^{-} \\ {[\mathfrak{b}]_{\pi}} & \text { otherwise. }\end{cases}
$$

This follows from Theorem 6.1 and Propositions 13.14 and 13.9, by noting that if $\pi \in \Pi_{\text {reg }}^{\tau}$ then $\delta_{(x, \pi)}=0$ unless $\mathfrak{b}_{\pi}=1$ and $x \in \mathrm{X}^{-}$.

Recall that any $\pi \in \Pi_{\text {gen }}^{\tau}$ can be written in the form

$$
\pi \simeq \sigma_{1} \times \cdots \times \sigma_{t}
$$

where $\sigma_{i}$ is the base-change of an essentially square integrable representation, and the $\sigma_{i}$ 's are unique up to permutation. Denote by $r=r(\pi)$ the number of indices $i$ such that $\sigma_{i}$ is itself essentially square integrable. Clearly $\mathfrak{b}_{\pi} \leq 2^{r}$ and $[\mathfrak{b}]_{\pi} \leq 2^{\max (r-1,0)}$. If $\pi \in \Pi_{\text {reg }}^{\tau}$ then equalities hold. In general however, $[\mathfrak{b}]_{\pi}$ may be much smaller. For instance, if $\pi$ is induced from the trivial character of $\mathrm{M}_{0}$ then $r(\pi)=n$ whereas $\left[\mathfrak{b}_{\pi}\right]=\left\lfloor\frac{n}{2}\right\rfloor+1$.

We apply Lemma D.1 and Corollary 13.15 to improve the lower bound obtained in Proposition 13.14 on the dimension of the space of local unitary periods of generic representations. For convenience, set

$$
2_{x}^{i}= \begin{cases}2^{i} & \text { if } i \geq 0, \\ 1 & \text { if } i=-1 \text { and } x \in \mathrm{X}^{+} \\ 0 & \text { if } i=-1 \text { and } x \in \mathrm{X}^{-}\end{cases}
$$

Corollary 13.16. - Let $\pi \in \Pi_{\text {gen }}^{\tau}$ and $x \in \mathrm{X}$. Then

$$
\operatorname{dim} \operatorname{Hom}_{\mathrm{G}^{x}}(\pi, \mathbf{G}) \geq 2_{x}^{r(\pi)-1} .
$$

Moreover, equality holds if $\pi \in \Pi_{\mathrm{reg}}^{\tau}$.

Conjecture 13.17. - For any $\pi \in \Pi_{\text {gen }}^{\tau}$ and $x \in \mathrm{X}$ we have an equality in $(\mathbf{1 3 . 5})$

13.5. Further remarks. - In the non-regular case, the naive upper bound on $\operatorname{dim} \operatorname{Hom}_{\mathrm{G}^{x}}(\pi, \mathbf{C})$ obtained from the Geometric Lemma is much coarser than the expected bound. For instance, in the case where $\pi$ is the principal series representation induced from the trivial character of $\mathrm{M}_{0}$, all $\mathrm{P}_{0}$-orbits are relevant their number is $\sum_{w \in \mathrm{S}_{n}: w^{2}=1} 2_{x}^{\left|\mathfrak{F}_{w}\right|-1}$ which is of course much larger than $2^{n-1}$. (Recall that $\mathfrak{F}_{w}$ is the set of fixed points of $w$.)

The following remark was communicated to us by Yiannis Sakellaridis. Suppose that $\mathrm{E} / \mathrm{F}$ is unramified. Then it follows from Hironaka's results [Hir99] that Conjecture 13.17 holds for unramified (generic) representations. Indeed, Hironaka showed that 
$\mathcal{S}(\mathrm{X})^{\mathrm{K}}=\mathcal{S}(\mathrm{X} / \mathrm{K})$ is a free module of rank $2^{n}$ over the Hecke algebra $\mathcal{H}=\mathcal{H}(\mathrm{G}, \mathrm{K})=$ $\mathcal{S}(\mathrm{K} \backslash \mathrm{G} / \mathrm{K})$ [ibid., Theorem 2]. Therefore, for any $\pi \in \Pi_{\text {unr }}$ we have

$$
\begin{aligned}
\mathcal{E}_{\mathrm{G}}\left(\mathrm{X}, \pi^{*}\right) & =\operatorname{Hom}_{\mathrm{G}}\left(\mathcal{S}(\mathrm{X}), \pi^{\vee}\right) \stackrel{\iota}{\longrightarrow} \operatorname{Hom}_{\mathcal{H}}\left(\mathcal{S}(\mathrm{X})^{\mathrm{K}},\left(\pi^{\vee}\right)^{\mathrm{K}}\right) \\
& \simeq \operatorname{Hom}_{\mathcal{H}}\left(\mathcal{H}^{2^{n}},\left(\pi^{\vee}\right)^{\mathrm{K}}\right) \simeq \mathbf{G}^{2^{n}} .
\end{aligned}
$$

Note that the restriction map $\iota$ is injective since if $f: \mathcal{S}(\mathrm{X}) \rightarrow \pi^{\vee}$ is a non-zero intertwining map then $f$ is onto, and therefore by averaging over $\mathbf{K}, \iota(f)$ is also onto. Thus, $\operatorname{dim} \mathcal{E}_{\mathrm{G}}\left(\mathrm{X}, \pi^{*}\right) \leq 2^{n}$. Using $(\mathbf{1 3 . 5})$ and Corollary 13.16 we get that if in addition $\pi$ is generic then $\iota$ is an isomorphism, $\operatorname{dim} \mathcal{E}_{\mathrm{G}}\left(\mathrm{X}, \pi^{*}\right)=2^{n}$ and $\operatorname{dim}_{\operatorname{Hom}_{\mathrm{G}^{x}}}(\pi, \mathbf{C})=2^{n-1}$ for all $x \in \mathrm{X}$. It would be interesting to generalize this type of argument to any generic representation.

13.6. Conclusion. - Let us summarize the main results obtained so far about the spaces $\mathcal{E}_{\mathrm{G}}\left(\mathrm{X}, \pi^{*}\right)$ and $\operatorname{Hom}_{\mathrm{G}^{x}}(\pi, \mathbf{C})$ and what still remains to be seen. The study of the space $\mathcal{E}_{\mathrm{G}}\left(\mathrm{X}, \pi^{*}\right)$ reduces to the case where $\pi$ is pure and Galois invariant. For irreducible representations of the form $\pi=\delta_{1} \times \cdots \times \delta_{k} \in \Pi^{\tau}$ with $\delta_{i}$ essentially square integrable we expect that

$$
\begin{aligned}
\operatorname{dim} \mathcal{E}_{\mathrm{G}}\left(\mathrm{X}, \pi^{*}\right) & =2^{\#\left\{i: \delta_{i} \in \Pi^{\tau}\right\}}, \\
\operatorname{dim} \operatorname{Hom}_{\mathrm{G}^{x}}(\pi, \mathbf{G}) & = \begin{cases}\left\lceil\frac{1}{2} \operatorname{dim} \mathcal{E}_{\mathrm{G}}\left(\mathrm{X}, \pi^{*}\right)\right\rceil & \text { if } \mathrm{G}^{x} \text { is quasi-split } \\
\left\lfloor\frac{1}{2} \operatorname{dim} \mathcal{E}_{\mathrm{G}}\left(\mathrm{X}, \pi^{*}\right)\right\rfloor & \text { otherwise. }\end{cases}
\end{aligned}
$$

This is known if $\mathrm{E} / \mathrm{F}$ and $\pi$ are unramified or if the Galois-invariant $\delta_{i}$ 's are distinct in which case an explicit basis for $\mathcal{E}_{\mathrm{G}}\left(\mathrm{X}, \pi^{*}\right)$ is given by $\left\{\alpha^{\pi^{\prime}}: \pi^{\prime} \in \mathcal{B}(\pi)\right\}$. In general we know the inequalities $\geq$ (even if $\delta_{i}$ are ladder representations) although it is not clear how to construct, even conjecturally, a nice basis for $\mathcal{E}_{\mathrm{G}}\left(\mathrm{X}, \pi^{*}\right)$ if $\pi \notin \Pi_{\mathrm{reg}}^{\tau}$.

Note that $(\mathbf{1 3 . 6 a})$ and $(\mathbf{1 3 . 6 b})$ hold at least if we twist the Galois-invariant $\delta_{i}$ 's by $\mid$ det $\left.\cdot\right|^{\lambda_{i}}$ for $\lambda_{i}$ 's outside finitely many 'hyperplanes' of the form $q^{\lambda_{i}-\lambda_{j}}=c$.

One may even wonder whether in general $\mathcal{E}_{\mathrm{G}}\left(\mathrm{X}, \pi_{1}^{*} \times \pi_{2}^{*}\right) \simeq \mathcal{E}_{\mathrm{G}_{n_{1}}}\left(\mathrm{X}_{n_{1}}, \pi_{1}^{*}\right) \otimes$ $\mathcal{E}_{\mathrm{G}_{n_{2}}}\left(\mathrm{X}_{n_{2}}, \pi_{2}^{*}\right)$ for any $\pi_{i} \in \Pi^{\mathrm{G}_{n_{i}}, \tau}, i=1,2$. Again, this holds upon twisting $\pi_{1}$ (or $\left.\pi_{2}\right)$ by $|\operatorname{det} \cdot|^{s}$ for $q^{s}$ outside a finite set and the inequality

$$
\operatorname{dim} \mathcal{E}_{\mathrm{G}}\left(\mathrm{X}, \pi_{1}^{*} \times \pi_{2}^{*}\right) \geq \operatorname{dim} \mathcal{E}_{\mathrm{G}_{n_{1}}}\left(\mathrm{X}_{n_{1}}, \pi_{1}^{*}\right) \operatorname{dim} \mathcal{E}_{\mathrm{G}_{n_{2}}}\left(\mathrm{X}_{n_{2}}, \pi_{2}^{*}\right)
$$

always holds.

Another aspect not discussed here is the computation of the local open periods on special vectors (such as new vectors). This will be useful to the analysis of the growth of unitary periods (cf. [LO07, Sar04]) as well as for possible arithmetic applications (cf. [CO07]). 


\section{Acknowledgements}

We are grateful to Akshay Venkatesh for calling our attention to a mistake in an earlier version of this paper. We also thank Yiannis Sakellaridis for pointing out to us that Hironaka's result gives the correct upper bound on multiplicities of local unitary periods for unramified principle series. We thank Avraham Aizenbud for sharing his insights on the Archimedean case which resulted in Appendix B. We thank Laurent Clozel for a useful suggestion in connection with the spectral comparison. We thank Michael Temkin and Yakov Varshavsky for helpful discussions. As usual, it is a pleasure to thank Hervé Jacquet for profitable discussions and inspiration and Jonathan Rogawski for constant encouragement. Thanks are also due to the referee for the careful reading of the paper and useful suggestions which prompted us to simplify some parts of the paper. Finally, we thank Joseph Bernstein, Eitan Sayag, Akshay Venkatesh and Nolan Wallach for their help with Appendix D.

Jonathan Rogawski passed away on September 27th 2011. We dedicate this work to his memory.

\section{Appendix A: Inner product for induced representations of $\mathrm{GL}_{n}$, by Erez Lapid and Omer Offen}

In this section let $\mathrm{G}=\mathrm{GL}_{n}(\mathrm{~F})$ where $\mathrm{F}$ is a local field and let $\mathcal{Q}=\mathcal{Q}_{n}$ be the mirabolic subgroup of $\mathrm{G}$, i.e., the stabilizer of $(0, \ldots, 0,1)$ under the right action of $\mathrm{G}$ on row vectors.

A.1 Canonical inner product. - Fix a non-trivial character $\psi$ of $\mathrm{F}$ and let $\pi \in \Pi_{\text {gen }}$. Recall that for simplicity we write $\mathcal{W}(\pi)=\mathcal{W}^{\psi}(\pi)$ and $\mathcal{W}\left(\pi^{\vee}\right)=\mathcal{W}^{\psi^{-1}}\left(\pi^{\vee}\right)$. It follows from the theory of Rankin-Selberg integrals developed by Jacquet, Piatetski-Shapiro and Shalika [JPSS83, JS90, Jac09] that the integral

$$
\begin{gathered}
\mathrm{I}\left(\mathrm{W}, \mathrm{W}^{\vee}, s\right)=\mathrm{L}\left(n, 1_{\mathrm{F}^{*}}\right) \int_{\mathrm{U}_{0} \backslash \mathcal{Q}_{n}} \mathrm{~W}(p) \mathrm{W}^{\vee}(p)|\operatorname{det} p|^{s} d p, \\
\mathrm{~W} \in \mathcal{W}(\pi), \mathrm{W}^{\vee} \in \mathcal{W}\left(\pi^{\vee}\right)
\end{gathered}
$$

converges for $\operatorname{Re} s \gg 0$ and admits a meromorphic continuation. Here, $\mathrm{L}\left(\cdot, 1_{\mathrm{F}^{*}}\right)$ is the Tate local L-factor attached to the trivial character of $\mathrm{F}^{*}$, which is included because of our measures convention. In the $p$-adic case, Bernstein showed that $\mathrm{I}\left(\mathrm{W}, \mathrm{W}^{\vee}, s\right)$ is holomorphic at $s=0$ and its value defines a non-degenerate $\mathrm{G}$-invariant pairing $[\cdot, \cdot]=$ $[\cdot, \cdot]_{\pi}$ between $\mathcal{W}(\pi)$ and $\mathcal{W}\left(\pi^{\vee}\right)$ [Ber84]. This was a consequence of his result that any $\mathcal{Q}_{n}$-invariant pairing is $\mathrm{G}$-invariant and the fact that the Kirillov model of $\pi$ contains the space of compactly supported functions on $\mathrm{U}_{0} \backslash \mathcal{Q}_{n}$. These results were subsequently 
proved in the Archimedean case by Baruch and Jacquet respectively ([Bar03], ${ }^{12}$ [Jac10, Proposition 5]). Therefore, $[\cdot, \cdot]$ is defined in the Archimedean case as well.

Note that the Rankin-Selberg zeta integral

$$
\begin{aligned}
& \mathrm{Z}\left(\mathrm{W}, \mathrm{W}^{\vee}, \Phi, s\right)=\int_{\mathrm{U}_{0} \backslash \mathrm{G}} \mathrm{W}(g) \mathrm{W}^{\vee}(g) \Phi((0, \ldots, 0,1) g)|\operatorname{det} g|^{s} d g, \\
& \Phi \in \mathcal{S}\left(\mathrm{F}^{n}\right)
\end{aligned}
$$

can be written as

$$
\begin{aligned}
& \mathrm{L}\left(n, 1_{\mathrm{F}^{*}}\right)^{-1} \int_{\mathcal{Q}_{n} \backslash \mathrm{G}} \mathrm{I}\left(\mathcal{W}(g, \pi) \mathrm{W}, \mathcal{W}\left(g, \pi^{\vee}\right) \mathrm{W}^{\vee}, s-1\right) \Phi((0, \ldots, 0,1) g) \\
& \quad \times|\operatorname{det} g|^{s} d g .
\end{aligned}
$$

In particular, since $\int_{\mathcal{Q}_{n} \backslash \mathrm{G}} \Phi((0, \ldots, 0,1) g)|\operatorname{det} g| d g=\mathrm{L}\left(n, 1_{\mathrm{F}^{*}}\right) \hat{\Phi}(0)$ we get

$$
\mathrm{Z}\left(\mathrm{W}, \mathrm{W}^{\vee}, \Phi, 1\right)=\left[\mathrm{W}, \mathrm{W}^{\vee}\right] \hat{\Phi}(0) \text {. }
$$

Note also that in the case where $\pi \in \Pi_{\text {gen }}$ is unitarizable we have $\mathcal{W}\left(\pi^{\vee}\right)=\{\overline{\mathrm{W}}$ : $\mathrm{W} \in \mathcal{W}(\pi)\}$ where $\overline{\mathrm{W}}(g)=\overline{\mathrm{W}(g)}, g \in \mathrm{G}$. In this case we have

$$
[\mathrm{W}, \overline{\mathrm{W}}]_{\pi}=\mathrm{L}\left(n, 1_{\mathrm{F}^{*}}\right) \int_{\mathrm{U}_{0} \backslash \mathcal{Q}_{n}}|\mathrm{~W}(p)|^{2} d p
$$

where the integral converges.

A.2 A remark by facquet on the square-integrable case. - In the case where $\pi \in \Pi_{\text {sqr }}$ the integral

$$
\left(\mathrm{W}, \mathrm{W}^{\vee}\right)=\int_{\mathrm{ZU}_{0} \backslash \mathrm{G}} \mathrm{W}(g) \mathrm{W}^{\vee}(g) d g
$$

converges, and by uniqueness, it is proportional to [W, $\left.\mathrm{W}^{\vee}\right]$. For completeness, we include the following result which was kindly communicated to us by Jacquet. (We will not use this result in the sequel.)

Lemma A.1 Jacquet. — With our normalization of measures, we have

$$
\left[\mathrm{W}, \mathrm{W}^{\vee}\right]=\frac{1}{n} \gamma\left(0, \pi^{\vee}, \mathrm{Ad} ; \psi^{-1}\right) \omega_{\pi}(-1)^{n-1} \mathrm{~L}\left(1,1_{\mathrm{F}^{*}}\right)\left(\mathrm{W}, \mathrm{W}^{\vee}\right)
$$

for any $\pi \in \Pi_{\mathrm{sqr}}$ and any $\mathrm{W} \in \mathcal{W}(\pi), \mathrm{W}^{\vee} \in \mathcal{W}\left(\pi^{\vee}\right)$.

\footnotetext{
${ }^{12}$ See $[$ AG09b] for a stronger statement.
} 
Proof. - Consider the local functional equation

$$
\begin{aligned}
\mathrm{Z}\left(\mathrm{W}^{\tilde{\theta}}, \mathrm{W}^{\vee \tilde{\theta}}, \hat{\Phi}, 1-s\right)= & \omega_{\pi}(-1)^{n-1} \gamma\left(s, \pi \times \pi^{\vee} ; \psi\right) \\
& \times \mathrm{Z}\left(\mathcal{W}\left(w_{0}, \pi\right) \mathrm{W}, \mathcal{W}\left(w_{0}, \pi^{\vee}\right) \mathrm{W}^{\vee}, \Phi, s\right)
\end{aligned}
$$

where $\mathrm{W}^{\tilde{\theta}}(g)=\mathrm{W}\left(w_{0}^{t} g^{-1} w_{0}\right)$. Write

$$
\gamma\left(s, \pi \times \pi^{\vee} ; \psi\right)=\gamma(s, \pi, \operatorname{Ad} ; \psi) \gamma\left(s, 1_{\mathrm{F}^{*}} ; \psi\right) .
$$

Thus, $\mathrm{Z}\left(\mathrm{W}^{\tilde{\theta}}, \mathrm{W}^{\vee \tilde{\theta}}, \hat{\Phi}, 1\right)$ is equal to

$$
\begin{aligned}
& \omega_{\pi}(-1)^{n-1} \gamma(0, \pi, \mathrm{Ad} ; \psi) \gamma^{\prime}\left(0,1_{\mathrm{F}^{*}} ; \psi\right) \\
& \quad \times \operatorname{Res}_{s=0} \mathrm{Z}\left(\mathcal{W}\left(w_{0}, \pi\right) \mathrm{W}, \mathcal{W}\left(w_{0}, \pi^{\vee}\right) \mathrm{W}^{\vee}, \Phi, s\right) .
\end{aligned}
$$

To compute the residue of the Rankin-Selberg integral at 0 we first recall the Tate integral

$$
\mathcal{Z}(\phi, s)=\int_{\mathrm{F}^{*}} \phi(t)|t|^{s} d^{*} t, \quad \phi \in \mathcal{S}(\mathrm{F})
$$

which converges for $\operatorname{Re} s>0$ and admits a meromorphic continuation with $\lim _{s \rightarrow 0} s \mathcal{Z}(\phi, s)=\frac{\mathrm{L}\left(1,1_{\mathrm{F}^{*}}\right)}{\gamma^{\prime}\left(0,1_{\left.\mathrm{F}^{*} ; \psi\right)}\right.} \phi(0)$. We can write

$$
\mathrm{Z}\left(\mathrm{W}, \mathrm{W}^{\vee}, \Phi, s\right)=\int_{\mathrm{ZU}_{0} \backslash \mathrm{G}} \mathrm{W}(g) \mathrm{W}^{\vee}(g) \mathcal{Z}\left(\phi_{g}, n s\right)|\operatorname{det} g|^{s} d g
$$

where $\phi_{g}(t)=\Phi((0, \ldots, 0, t) g)$. Note that $\left|s \mathcal{Z}\left(\phi_{g}, n s\right)\right||\operatorname{det} g|^{s}$ is bounded near $s=0$ by a constant multiple of $e^{s\langle\varpi, \mathrm{H}(g)\rangle}$ where $\varpi$ is the fundamental weight corresponding to the parabolic $\mathcal{Q}_{n} \mathrm{Z}$. On the other hand by easy estimates on the Whittaker function (e.g., [LM09a, Wal92]) the integral

$$
\int_{\mathrm{U}_{0} \backslash \mathrm{G}}|\mathrm{W}(g)|^{2} e^{s\langle\varpi, \mathrm{H}(g)\rangle} d g
$$

converges in a neighborhood of $s=0$. Therefore, we can take the residue at $s=0$ inside the integral of (A.2) to obtain

$$
\operatorname{Res}_{s=0} \mathrm{Z}\left(\mathrm{W}, \mathrm{W}^{\vee}, \Phi, s\right)=\frac{\mathrm{L}\left(1,1_{\mathrm{F}^{*}}\right)}{n \gamma^{\prime}\left(0,1_{\mathrm{F}^{*}} ; \psi\right)} \Phi(0) \int_{\mathrm{ZU}_{0} \backslash \mathrm{G}} \mathrm{W}(g) \mathrm{W}^{\vee}(g) d g .
$$

Thus,

$$
\begin{aligned}
{\left[\mathrm{W}^{\tilde{\theta}}, \mathrm{W}^{\vee \tilde{\theta}}\right]=} & \frac{1}{n} \gamma(0, \pi, \mathrm{Ad} ; \psi) \omega_{\pi}(-1)^{n-1} \mathrm{~L}\left(1,1_{\mathrm{F}^{*}}\right) \\
& \times\left(\mathcal{W}\left(w_{0}, \pi\right) \mathrm{W}, \mathcal{W}\left(w_{0}, \pi^{\vee}\right) \mathrm{W}^{\vee}\right) .
\end{aligned}
$$


Since $\left(\mathcal{W}\left(w_{0}, \pi\right) \mathrm{W}, \mathcal{W}\left(w_{0}, \pi^{\vee}\right) \mathrm{W}^{\vee}\right)=\left(\mathrm{W}, \mathrm{W}^{\vee}\right)=\left(\mathrm{W}^{\tilde{\theta}}, \mathrm{W}^{\vee \tilde{\theta}}\right)$ we get

$$
\left[\mathrm{W}^{\tilde{\theta}}, \mathrm{W}^{\vee \tilde{\theta}}\right]=\frac{1}{n} \gamma(0, \pi, \mathrm{Ad} ; \psi) \omega_{\pi}(-1)^{n-1} \mathrm{~L}\left(1,1_{\mathrm{F}^{*}}\right)\left(\mathrm{W}^{\tilde{\theta}}, \mathrm{W}^{\vee \tilde{\theta}}\right) .
$$

This is equivalent to the statement of the Lemma.

A.3 Let us analyze a few properties of $[\cdot, \cdot]$. Clearly,

$$
\left[\mathrm{W} \cdot \chi, \mathrm{W}^{\vee} \cdot \chi^{-1}\right]_{\pi \cdot \chi}=\left[\mathrm{W}, \mathrm{W}^{\vee}\right]_{\pi}
$$

for any $\mathrm{W} \in \mathcal{W}(\pi), \mathrm{W}^{\vee} \in \mathcal{W}\left(\pi^{\vee}\right)$ and a character $\chi$ of $\mathrm{F}^{*}$.

Suppose now that $\pi \in \Pi_{\text {unr }}$ and $\psi$ has conductor $\mathcal{O}_{\mathrm{F}}$. Let $\mathrm{W}_{0}^{\pi} \in \mathcal{W}(\pi)$ be the spherical Whittaker function normalized by $\mathrm{W}_{0}^{\pi}(e)=1$. Then we have [JS81b]

$$
\left[\mathrm{W}_{0}^{\pi}, \mathrm{W}_{0}^{\pi^{\vee}}\right]_{\pi}=\mathrm{L}\left(1, \pi \times \pi^{\vee}\right) .
$$

We will now study the behavior of $[\cdot, \cdot]$ with respect to induction. Let $\mathrm{P}=\mathrm{MU}$ be a standard parabolic subgroup of $\mathrm{G}, \sigma \in \Pi_{\text {gen }}^{\mathrm{M}}$ and $\lambda \in \mathfrak{a}_{\mathrm{M}, \mathbf{G}}^{*} \cdot$ Let $(\cdot, \cdot)_{\mathrm{M}}=\mathrm{I}_{\mathrm{M}}\left([\cdot, \cdot]_{\sigma}\right)$ be the pairing between the induced representations $\mathrm{I}(\mathcal{W}(\sigma), \lambda)$ and $\mathrm{I}\left(\mathcal{W}\left(\sigma^{\vee}\right),-\lambda\right)$ given by

$$
\left(\varphi, \varphi^{\vee}\right)_{\mathrm{M}}=\int_{\mathrm{P} \backslash \mathrm{G}}\left[\varphi(g), \varphi^{\vee}(g)\right]_{\sigma} d g
$$

where $[\cdot, \cdot]_{\sigma}$ is defined with respect to $\mathrm{M}$. Let $\mathbf{W}(\varphi, \sigma, \lambda)$ be the Jacquet integral defined in Section 1.4 and recall the convention $\mathbf{W}\left(\varphi^{\vee}, \sigma^{\vee},-\lambda\right)=\mathbf{W}^{\psi^{-1}}\left(\varphi^{\vee}, \sigma^{\vee},-\lambda\right)$ for $\varphi \in$ $\mathrm{I}\left(\mathcal{W}\left(\sigma^{\vee}\right)\right)=\mathrm{I}\left(\mathcal{W}^{\psi^{-1}}\left(\sigma^{\vee}\right)\right)$.

Proposition A.2. - We have

$$
\left(\varphi, \varphi^{\vee}\right)_{\mathrm{M}}=\left[\mathbf{W}(\varphi, \sigma, \lambda), \mathbf{W}\left(\varphi^{\vee}, \sigma^{\vee},-\lambda\right)\right]
$$

for all $\varphi \in \mathrm{I}(\mathcal{W}(\sigma)), \varphi^{\vee} \in \mathrm{I}\left(\mathcal{W}\left(\sigma^{\vee}\right)\right)$ and $\lambda \in \mathfrak{a}_{\mathrm{M}, \mathbf{C}}^{*}$ such that $\mathrm{I}(\sigma, \lambda)$ is irreducible.

Remark A.3. - In the principal series case (i.e., when P is the Borel subgroup) this follows by analytic continuation from [LO07, Proposition 1]. The extension to the general case provided here follows the same reasoning, but is technically more complicated.

Proof. - We first reduce to the case where $\sigma$ is unitarizable (or even, $\sigma \in \Pi_{\text {usqr }}$ is square integrable). Indeed, suppose that $\sigma=\mathrm{I}_{\mathrm{L}}^{\mathrm{M}}(\delta, \mu)$ where $\delta \in \Pi_{\text {usqr }}^{\mathrm{L}}$. Let $\xi \in$ $\mathrm{I}_{\mathrm{M}}(\mathcal{W}(\sigma))$ and $\xi^{\vee} \in \mathrm{I}_{\mathrm{M}}\left(\mathcal{W}\left(\sigma^{\vee}\right)\right)$. We may write $\xi=\mathbf{W}^{\mathrm{M}}(\varphi, \delta, \mu)$ where $\varphi \in \mathrm{I}_{\mathrm{L}}(\mathcal{W}(\delta))$. Similarly, write $\xi^{\vee}=\mathbf{W}^{\mathrm{M}}\left(\varphi^{\vee}, \delta^{\vee},-\mu\right)$ where $\varphi^{\vee} \in \mathrm{I}_{\mathrm{L}}\left(\mathcal{W}\left(\delta^{\vee}\right)\right)$. Thus, $\mathbf{W}_{\mathrm{M}}(\xi, \sigma, \lambda)=$ $\mathbf{W}_{\mathrm{L}}(\varphi, \delta, \mu+\lambda)$ and $\mathbf{W}_{\mathrm{M}}\left(\xi^{\vee}, \sigma^{\vee},-\lambda\right)=\mathbf{W}_{\mathrm{L}}\left(\varphi^{\vee}, \delta^{\vee},-\mu-\lambda\right)$. By assumption (applied to $\delta$ and $\mathrm{M}$ instead of $\mathrm{G})$ we have

$$
\left[\xi(g), \xi^{\vee}(g)\right]_{\sigma}=\int_{\mathrm{Q} \backslash \mathrm{P}} \delta_{\mathrm{P}}(p)^{-1}\left[\varphi(p g), \varphi^{\vee}(p g)\right]_{\delta} d p
$$


for all $g \in \mathrm{G}$. Hence,

$$
\begin{aligned}
\left(\xi, \xi^{\vee}\right)_{\mathrm{M}} & =\int_{\mathrm{P} \backslash \mathrm{G}}\left[\xi(g), \xi^{\vee}(g)\right]_{\sigma} d g=\int_{\mathrm{P} \backslash \mathrm{G}} \int_{\mathrm{Q} \backslash \mathrm{P}} \delta_{\mathrm{P}}(p)^{-1}\left[\varphi(p g), \varphi^{\vee}(p g)\right]_{\delta} d p d g \\
& =\int_{\mathrm{Q} \backslash \mathrm{G}}\left[\varphi(g), \varphi^{\vee}(g)\right]_{\delta} d g=\left(\varphi, \varphi^{\vee}\right)_{\mathrm{L}} .
\end{aligned}
$$

Once again by assumption (applied to $\delta$ and $\mathrm{G}$ ) this equals

$$
\left[\mathbf{W}(\varphi, \delta, \lambda+\mu), \mathbf{W}\left(\varphi^{\vee}, \delta^{\vee},-\lambda-\mu\right)\right]=\left[\mathbf{W}(\xi, \sigma, \lambda), \mathbf{W}\left(\xi^{\vee}, \sigma^{\vee},-\lambda\right)\right]
$$

as required.

From now on, suppose that $\sigma \in \Pi_{\text {gen }}^{\mathrm{M}}$ is unitarizable. In this case $\mathcal{W}\left(\sigma^{\vee}\right)=\{\overline{\mathrm{W}}$ : $\mathrm{W} \in \mathcal{W}(\sigma)\}$ where $\overline{\mathrm{W}}(m)=\overline{\mathrm{W}(m)}$ for all $m \in \mathrm{M}$ and $\mathrm{I}\left(\mathcal{W}\left(\sigma^{\vee}\right)\right)=\{\bar{\varphi}: \varphi \in \mathrm{I}(\mathcal{W}(\sigma))\}$ where $\bar{\varphi}(g)=\overline{\varphi(g)}$. Thus, we can take $\varphi^{\vee}=\bar{\varphi}$. Note that $\overline{\mathbf{W}(\varphi, \sigma, \lambda)}=\mathbf{W}\left(\varphi^{\vee}, \sigma^{\vee}, \bar{\lambda}\right)$.

We first observe that the right-hand side of $(\mathbf{A . 5})$ is holomorphic whenever $\mathrm{I}(\sigma, \lambda)$ is irreducible. Indeed, it is known that $\frac{\mathrm{Z}\left(\mathbf{W}(\varphi, \sigma, \lambda), \mathbf{W}\left(\varphi^{\vee}, \sigma^{\vee},-\lambda\right), \Phi, s\right)}{\mathrm{L}\left(s, \mathrm{I}(\sigma, \lambda) \times \mathrm{I}\left(\sigma^{\vee},-\lambda\right)\right)}$ is entire in $(\lambda, s) \in \mathfrak{a}_{\mathrm{M}, \mathbf{C}}^{*} \times \mathbf{C}$ [JPSS83, Jac04a]. In particular, it follows from $(\mathbf{1 . 1 2})$ that $\mathrm{Z}\left(\mathbf{W}(\varphi, \sigma, \lambda), \mathbf{W}\left(\varphi^{\vee}, \sigma^{\vee},-\lambda\right)\right.$, $\Phi, s)$ is holomorphic at $(\lambda, 1)$ whenever $\mathrm{I}(\sigma, \lambda)$ is irreducible. However, $\mathrm{Z}(\mathbf{W}(\varphi, \sigma, \lambda)$, $\left.\mathbf{W}\left(\varphi^{\vee}, \sigma^{\vee},-\lambda\right), \Phi, 1\right)$ is $\hat{\Phi}(0)$ times the right-hand side of (A.5). Our claim follows.

By analytic continuation, it is enough to consider the case $\lambda \in \mathrm{ia}_{\mathrm{M}}^{*}$. In fact, by twisting $\sigma$ we can also assume without loss of generality that $\lambda=0$.

By induction on $n$, we will reduce further to the case where $\mathrm{P}$ is maximal and at the same time to the existence of a positive constant $c_{\mathrm{M}}^{\mathrm{G}}$ depending only on normalization of measures, so that

$$
\left(\varphi, \varphi^{\vee}\right)_{\mathrm{M}}=c_{\mathrm{M}}^{\mathrm{G}}\left[\mathbf{W}(\varphi, 0), \mathbf{W}\left(\varphi^{\vee}, 0\right)\right] .
$$

Let $\mathrm{Q}$ be a maximal parabolic containing $\mathrm{P}$ and let $\delta=\mathrm{I}_{\mathrm{M}}^{\mathrm{L}}(\mathcal{W}(\sigma))$. Let $\varphi \in \mathrm{I}_{\mathrm{M}}(\mathcal{W}(\sigma))$ and let $\xi=\mathbf{W}^{\mathrm{L}}(\varphi, \sigma, 0)$. Then $\xi \in \mathrm{I}_{\mathrm{L}}(\mathcal{W}(\delta))$ and $\mathbf{W}_{\mathrm{L}}(\xi, \delta, 0)=\mathbf{W}_{\mathrm{M}}(\varphi, \sigma, 0)$. We have

$$
\begin{aligned}
\left(\varphi, \varphi^{\vee}\right)_{\mathrm{M}} & =\int_{\mathrm{P} \backslash \mathrm{G}}\left[\varphi(g), \varphi^{\vee}(g)\right]_{\mathrm{M}} d g \\
& =\int_{\mathrm{Q} \backslash \mathrm{G}} \int_{\mathrm{P} \backslash \mathrm{Q}} \delta_{\mathrm{Q}}(q)^{-1}\left[\varphi(q g), \varphi^{\vee}(q g)\right]_{\mathrm{M}} d q d g .
\end{aligned}
$$

By induction hypothesis, the inner integral is

$$
c_{\mathrm{M}}^{\mathrm{L}}\left[\xi(g), \xi^{\vee}(g)\right]_{\delta} .
$$

Thus,

$$
\begin{aligned}
\left(\varphi, \varphi^{\vee}\right)_{\mathrm{M}} & =c_{\mathrm{M}}^{\mathrm{L}}\left(\xi, \xi^{\vee}\right)_{\mathrm{L}}=c_{\mathrm{M}}^{\mathrm{L}} c_{\mathrm{L}}^{\mathrm{G}}\left[\mathbf{W}(\xi, \delta, 0), \mathbf{W}\left(\xi^{\vee}, \delta^{\vee}, 0\right)\right] \\
& =c_{\mathrm{M}}^{\mathrm{L}} c_{\mathrm{L}}^{\mathrm{G}}\left[\mathbf{W}(\varphi, \sigma, 0), \mathbf{W}\left(\varphi^{\vee}, \sigma^{\vee}, 0\right)\right],
\end{aligned}
$$


where for the second equality we used the assumption for the maximal parabolic case. Thus, knowing (A.6) for a maximal parabolic implies it for a general parabolic and implies further that whenever $\mathrm{P} \subseteq \mathrm{Q} \subseteq \mathrm{G}$ we have

$$
c_{\mathrm{M}}^{\mathrm{G}}=c_{\mathrm{M}}^{\mathrm{L}} c_{\mathrm{L}}^{\mathrm{G}}
$$

By [LO07, Proposition 1] we know that $c_{\mathrm{M}_{0}}^{\mathrm{G}}=1$. We therefore obtain $c_{\mathrm{M}}^{\mathrm{G}}=c_{\mathrm{M}_{0}}^{\mathrm{G}}\left(c_{\mathrm{M}_{0}}^{\mathrm{M}}\right)^{-1}=1$ for all M. For the rest of the proof we do not need to worry about normalization of measures.

Suppose now that $\mathrm{P}$ is maximal of type $\left(n_{1}, n_{2}\right)$. We will prove the assertion by applying Fourier transform $n_{1}$ times to functions on $n_{2}$-dimensional vector spaces. Write $\mathrm{M}=\mathrm{M}_{1} \times \mathrm{M}_{2}$ where $\mathrm{M}_{1} \simeq \mathrm{GL}_{n_{1}}$ in the upper diagonal block and $\mathrm{M}_{2} \simeq \mathrm{GL}_{n_{2}}$ in the lower diagonal block. Let $w=w_{\mathrm{M}}^{-1}=\left({ }_{\mathrm{I}_{n_{2}}}^{\mathrm{I}_{n_{1}}}\right)$ and let $\mathrm{P}^{\prime}=\mathrm{M}^{\prime} \mathrm{U}^{\prime}$ be the maximal parabolic of type $\left(n_{2}, n_{1}\right)$ so that $\mathbf{M}^{\prime}=w^{-1} \mathbf{M} w=\mathbf{M}_{2}^{\prime} \times \mathbf{M}_{1}^{\prime}$ where $\mathbf{M}_{i}^{\prime}=w^{-1} \mathbf{M}_{i} w, i=1,2$. Let $\mathrm{U}_{i}^{\prime}$ be the group of upper unitriangular matrices in $\mathrm{M}_{i}^{\prime}, i=1,2$ and $\tilde{\mathcal{Q}}_{i}^{\prime}$ the mirabolic subgroup of $\mathrm{M}_{i}^{\prime}, i=1,2$. We similarly define the analogous subgroups $\mathrm{U}_{i}=w \mathrm{U}_{i}^{\prime} w^{-1}$ and $\tilde{\mathcal{Q}}_{i}=w \tilde{\mathcal{Q}}_{i}^{\prime} w^{-1}$ of $\mathrm{M}_{i}, i=1,2$.

We can identify $\mathrm{U}^{\prime}$ with the additive group of matrices of size $n_{2} \times n_{1}$. For $i=$ $n_{2}+1, \ldots, n$ let

$$
\mathrm{C}_{i}=\left\{\mathrm{I}_{n}+\xi: \xi \text { a column vector of size } n_{2} \text { in the } i \text {-th column }\right\} \subseteq \mathrm{U}^{\prime}
$$

and let $\mathrm{X}_{i}=\mathrm{C}_{i+1} \cdots \mathrm{C}_{n}, i=n_{2}, \ldots, n$. In particular, $\mathrm{X}_{n_{2}}=\mathrm{U}^{\prime}$. Similarly, let

$$
\mathrm{R}_{i}=\left\{\mathrm{I}_{n}+\xi: \xi \text { a row vector of size } n_{2} \text { in the } i \text {-th row }\right\} \subseteq{ }^{t} \mathrm{U}^{\prime}
$$

and $\mathrm{Y}_{i}=\mathrm{R}_{n_{2}+1} \cdots \mathrm{R}_{i-1}$. We have a pairing between $\mathrm{C}_{i}$ and $\mathrm{R}_{i-1}$.

Note that $\mathrm{X}_{i}$ and $\mathrm{Y}_{i}$ are both normalized by $\mathrm{M}_{2}^{\prime} \mathrm{U}_{1}^{\prime}$ and $\left[\mathrm{X}_{i-1}, \mathrm{Y}_{i}\right] \subseteq \mathrm{U}_{1}^{\prime}$. In particular, $\mathrm{V}_{i}=\mathrm{U}_{1}^{\prime} \mathrm{X}_{i} \mathrm{Y}_{i}$ and $\mathrm{V}_{i}^{\prime}=\mathrm{U}_{1}^{\prime} \mathrm{X}_{i-1} \mathrm{Y}_{i}$ are unipotent groups.

Define the groups

$$
\mathrm{S}_{i}= \begin{cases}\mathrm{M}_{2}^{\prime} \mathrm{V}_{i} & i>n_{2} \\ \tilde{\mathcal{Q}}_{2}^{\prime} \mathrm{U}_{1}^{\prime} \mathrm{U}^{\prime} & i=n_{2}\end{cases}
$$

Note that for $i>n_{2}$, the modulus function $\delta_{i}$ of $\mathrm{S}_{i}$ is $|\operatorname{det}|^{n+n_{2}+1-2 i}$. For $i>n_{2}$ let $\psi_{i}$ be the character on $\mathrm{V}_{i}$ which is trivial on $\mathrm{X}_{i} \mathrm{Y}_{i}$ and coincides with $\psi$ on $\mathrm{U}_{1}^{\prime}$. We also define

$$
\mathrm{S}_{i}^{\prime}=\mathrm{M}_{2}^{\prime} \mathrm{V}_{i}^{\prime}, \quad i>n_{2}
$$

so that $\mathrm{S}_{i}^{\prime}=\mathrm{C}_{i} \mathrm{~S}_{i}$ for $i>n_{2}$ and $\mathrm{S}_{i}^{\prime}=\mathrm{R}_{i-1} \mathrm{~S}_{i-1}$ for $i>n_{2}+1$. It follows that the modulus function $\delta_{i}^{\prime}$ on $\mathrm{S}_{i}^{\prime}$ satisfies $\left.\delta_{i}^{\prime}\right|_{\mathrm{S}_{i}}=\delta_{i}|\operatorname{det}|$ for $i>n_{2}$ and $\left.\delta_{i}^{\prime}\right|_{\mathrm{S}_{i-1}}=\delta_{i-1}|\operatorname{det}|^{-1}$ for $i>n_{2}+1$. 
Write $\sigma=\sigma_{1} \otimes \sigma_{2}$ with $\sigma_{i} \in \Pi_{\text {gen }}^{\mathrm{G}_{n_{i}}}$ and view $\sigma_{2}$ also as a representation of $\mathbf{M}_{2}^{\prime}$. We define representations of $\mathcal{Q}_{n}$ as follows

$$
\mathcal{A}_{i}= \begin{cases}\mathrm{L}^{2}-\operatorname{Ind}_{\mathrm{S}_{i}}^{\mathcal{Q}_{n}}\left(\overline{\mathcal{W}\left(\sigma_{2}\right)} \otimes \psi_{i}\right) & i=n_{2}+1, \ldots, n, \\ \mathrm{~L}^{2}-\operatorname{Ind}_{\mathrm{U}_{0}}^{\mathcal{Q}_{n}} \psi & i=n_{2},\end{cases}
$$

where $\overline{\mathcal{W}\left(\sigma_{2}\right)}$ denotes the completion of $\mathcal{W}\left(\sigma_{2}\right)$ with respect to the inner product $\|\mathrm{W}\|_{\mathcal{W}\left(\sigma_{2}\right)}^{2}=[\mathrm{W}, \overline{\mathrm{W}}]_{\sigma_{2}}$ and the prefix $\mathrm{L}^{2}$ indicates $\mathrm{L}^{2}$-induction. Thus, for $i>n_{2}$

$$
\begin{gathered}
\mathcal{A}_{i}=\left\{\varphi: \mathcal{Q}_{n} \rightarrow \overline{\mathcal{W}\left(\sigma_{2}\right)} \text { measurable }: \varphi(m v g)\right. \\
=\psi_{i}(v)\left(\frac{\delta_{i}(m)}{|\operatorname{det} m|}\right)^{\frac{1}{2}} \overline{\mathcal{W}\left(\sigma_{2}\right)}(m)[\varphi(g)] \\
\quad \text { for all } g \in \mathcal{Q}_{n}, m \in \mathrm{M}_{2}^{\prime}, v \in \mathrm{V}_{i}, \\
\left.\|\varphi\|_{i}^{2}:=\int_{\mathrm{S}_{i} \backslash \mathcal{Q}_{n}}\|\varphi(g)\|_{\overline{\mathcal{W}\left(\sigma_{2}\right)}}^{2} d g<\infty\right\} /\left\{\|\cdot\|_{i}=0\right\} .
\end{gathered}
$$

The smooth part of $\mathcal{A}_{i}$ is contained in the smooth induction $\operatorname{Ind}_{\mathrm{S}_{i}}^{\mathcal{Q}_{n}}\left(\mathcal{W}\left(\sigma_{2}\right) \otimes \psi_{i}\right)$ consisting of smooth functions $\varphi$ with values in $\mathcal{W}\left(\sigma_{2}\right)$ satisfying the equivariance property above [Pou72]. Similarly for $i=n_{2}$.

Using induction by stages we have

$$
\begin{aligned}
\mathcal{A}_{i} & =\mathrm{L}^{2}-\operatorname{Ind}_{\mathrm{S}_{i}^{\prime}}^{\mathcal{O}_{n}}\left(\mathrm{~L}^{2}-\operatorname{Ind}_{\mathrm{S}_{i}}^{\mathrm{S}_{i}^{\prime}}\left(\overline{\mathcal{W}\left(\sigma_{2}\right)} \otimes \psi_{i}\right)\right), \quad i>n_{2}, \\
\mathcal{A}_{i-1} & =\mathrm{L}^{2}-\operatorname{Ind}_{\mathrm{S}_{i}^{\prime}}^{\mathcal{O}_{n}}\left(\mathrm{~L}^{2}-\operatorname{Ind}_{\mathrm{S}_{i-1}}^{\mathrm{S}_{i}^{\prime}}\left(\overline{\mathcal{W}\left(\sigma_{2}\right)} \otimes \psi_{i}\right)\right), \quad i>n_{2}+1, \\
\mathcal{A}_{n_{2}} & =\mathrm{L}^{2}-\operatorname{Ind}_{\mathrm{S}_{n_{2}+1}^{\prime}}^{\mathcal{O}_{n}}\left(\mathrm{~L}^{2}-\operatorname{Ind}_{\mathrm{U}_{0}} \mathrm{~S}_{\mathrm{S}_{2}+1}^{\prime} \psi\right) .
\end{aligned}
$$

For any $i>n_{2}$ the restriction to $\mathrm{C}_{i}$ identifies $\left.\mathrm{L}^{2}-\operatorname{Ind}_{\mathrm{S}_{i}}^{\mathrm{S}_{i}^{\prime}} \overline{\mathcal{W}\left(\sigma_{2}\right)} \otimes \psi_{i}\right)$ with $\mathrm{L}^{2}\left(\mathrm{C}_{i}, \overline{\mathcal{W}\left(\sigma_{2}\right)}\right)$. Similarly for any $i>n_{2}+1$ the restriction to $\mathrm{R}_{i-1}$ identifies $\mathrm{L}^{2}-\operatorname{Ind}_{\mathrm{S}_{i-1}}^{\mathrm{S}_{i}^{\prime}}\left(\overline{\mathcal{W}\left(\sigma_{2}\right)} \otimes \psi_{i-1}\right)$ with $\mathrm{L}^{2}\left(\mathrm{R}_{i-1}, \overline{\mathcal{W}\left(\sigma_{2}\right)}\right)$.

Lemma A.4. - Under these identifications, the Fourier transform

$$
\mathrm{L}^{2}\left(\mathrm{G}_{i}, \overline{\mathcal{W}\left(\sigma_{2}\right)}\right) \rightarrow \mathrm{L}^{2}\left(\mathrm{R}_{i-1}, \overline{\mathcal{W}\left(\sigma_{2}\right)}\right)
$$

induces a unitary equivalence of representations

$$
\mathcal{F}_{i}: \mathrm{L}^{2}-\operatorname{Ind}_{\mathrm{S}_{i}}^{\mathrm{S}_{i}^{\prime}}\left(\overline{\mathcal{W}\left(\sigma_{2}\right)} \otimes \psi_{i}\right) \rightarrow \mathrm{L}^{2}-\operatorname{Ind}_{\mathrm{S}_{i-1}}^{\mathrm{S}_{i}^{\prime}}\left(\overline{\mathcal{W}\left(\sigma_{2}\right)} \otimes \psi_{i-1}\right), \quad i>n_{2}+1
$$


Similarly, for $i=n_{2}+1$ we have a unitary equivalence of representations

$$
\mathcal{F}_{n_{2}+1}: \mathrm{L}^{2}-\operatorname{Ind}_{\mathrm{S}_{n_{2}+1}}^{\mathrm{S}_{n_{2}+1}^{\prime}}\left(\overline{\mathcal{W}\left(\sigma_{2}\right)} \otimes \psi_{n_{2}+1}\right) \rightarrow \mathrm{L}^{2}-\operatorname{Ind}_{\mathrm{U}_{0}}^{\mathrm{S}_{n_{2}+1}^{\prime}} \psi_{0}
$$

given by

$$
\left[\mathcal{F}_{n_{2}+1} \varphi\right](v m)=\psi_{0}(v)|\operatorname{det} m|^{\frac{1}{2}} \hat{\varphi}\left(\chi_{m}\right)(m), \quad m \in \mathrm{M}_{2}^{\prime}, v \in \mathrm{V}_{n_{2}+1}^{\prime}=\mathrm{U}_{1}^{\prime} \mathrm{U}^{\prime}
$$

where $\chi_{m}$ is the character of $\mathrm{C}_{n_{2}+1}$ given by $\chi_{m}(c)=\psi\left(m_{c m}^{-1}\right)$, and $\hat{\varphi}\left(\chi_{m}\right)=\int_{\mathrm{C}_{n_{2}+1}} \varphi(c) \chi_{m}(c) d c$ denotes the Fourier transform of $\varphi$.

Proof. - Consider first the case $i>n_{2}+1$. Suppose that $\left.f_{1} \in \mathrm{L}^{2}-\operatorname{Ind}_{\mathrm{S}_{i}}^{\mathrm{S}_{i}^{\prime}} \overline{\left(\mathcal{W}\left(\sigma_{2}\right)\right.} \otimes \psi_{i}\right)$ and $f_{2} \in \mathrm{L}^{2}-\operatorname{Ind}_{\mathrm{S}_{i-1}}^{\mathrm{S}_{i}^{\prime}}\left(\overline{\mathcal{W}\left(\sigma_{2}\right)} \otimes \psi_{i-1}\right)$ are such that the Fourier transform of $\left.f_{1}\right|_{\mathrm{C}_{i}}$ is $\left.f_{2}\right|_{\mathrm{R}_{i-1}}$. We have to check that for any $s \in \mathrm{S}_{i}^{\prime}$ the Fourier transform of $f_{1}^{s}:=f_{1}(\cdot s)$ (as a function of $\mathrm{G}_{i}$ ) is $f_{2}^{s}:=f_{2}(\cdot s)$ (as a function on $\mathrm{R}_{i-1}$ ). We check this separately for the cases $s \in \mathrm{X}_{i}$, $s \in \mathrm{Y}_{i-1}, s \in \mathrm{U}_{1}^{\prime}, s \in \mathbf{C}_{i}, s \in \mathbf{R}_{i-1}$ and $s \in \mathbf{M}_{2}^{\prime}$. In the first three cases $f_{1}^{s}=f_{1}$ and $f_{2}^{s}=f_{2}$ because $\psi_{i-1}$ is trivial on $\left[\mathrm{X}_{i} \mathrm{U}_{1}^{\prime}, \mathrm{R}_{i-1}\right]$ and $\psi_{i}$ is trivial on $\left[\mathrm{U}_{1}^{\prime} \mathrm{Y}_{i-1}, \mathrm{C}_{i}\right]$. In the case $s \in \mathrm{C}_{i}$, $f_{1}^{s}$ is the translate of $\left.f_{1}\right|_{\mathrm{C}_{i}}$ by $s$ and $f_{2}^{s}(r)=\psi_{i-1}([r, s]) f_{2}(r)$ for any $r \in \mathrm{R}_{i-1}$. Similarly, in the case $s \in \mathbf{R}_{i-1}, f_{1}^{s}(c)=\psi_{i}([c, s]) f_{1}(c), c \in \mathbf{C}_{i}$ and $f_{2}^{s}$ is the translate of $f_{2}$ by $s$. Finally, for $s \in \mathbf{M}_{2}^{\prime}, f_{1}^{s}(c)=|\operatorname{det} s|^{-\frac{1}{2}} \sigma_{2}(s) f_{1}\left(s^{-1} c s\right)$ and $f_{2}^{s}(r)=|\operatorname{det} s|^{\frac{1}{2}} \sigma_{2}(s) f_{2}\left(s^{-1} r s\right)$. Thus, all cases follow from standard properties of the Fourier transform.

In the case $i=n_{2}+1$, in order to check that $\mathcal{F}_{n_{2}+1}$ is an isometry (and well-defined) we compute

$$
\begin{aligned}
\left\|\mathcal{F}_{n_{2}+1} \varphi\right\|_{\mathrm{L}^{2}\left(\mathrm{U}_{2}^{\prime} \backslash \mathrm{M}_{2}^{\prime}\right)}^{2} & =\int_{\mathrm{U}_{2}^{\prime} \backslash \mathrm{M}_{2}^{\prime}}\left|\hat{\varphi}\left(\chi_{m}\right)(m)\right|^{2}|\operatorname{det} m| d m \\
& =\int_{\tilde{\mathcal{Q}}_{n_{2}}^{\prime} \backslash \mathrm{M}_{2}^{\prime}} \int_{\mathrm{U}_{2}^{\prime} \backslash \tilde{\mathcal{Q}}_{n_{2}}^{\prime}}\left|\hat{\varphi}\left(\chi_{m}\right)(p m)\right|^{2}|\operatorname{det} m| d p d m \\
& =\int_{\tilde{\mathcal{Q}}_{n_{2}}^{\prime} \backslash \mathrm{M}_{2}^{\prime}}\left\|\sigma_{2}(m) \hat{\varphi}\left(\chi_{m}\right)\right\|_{\mathcal{W}\left(\sigma_{2}\right)}^{2}|\operatorname{det} m| d m \\
& =\int_{\tilde{\mathcal{Q}}_{n_{2}}^{\prime} \backslash \mathrm{M}_{2}^{\prime}}\left\|\hat{\varphi}\left(\chi_{m}\right)\right\|_{\overline{\mathcal{W}\left(\sigma_{2}\right)}}^{2}|\operatorname{det} m| d m \\
& =\int_{\mathrm{C}_{n_{2}+1}}\|\varphi(r)\|_{\mathcal{\mathcal { W } ( \sigma _ { 2 } )}}^{2} d r=\|\varphi\|^{2} .
\end{aligned}
$$

Finally, it is straightforward to check that $\mathcal{F}_{n_{2}+1}$ is an intertwining map of representations of $\mathrm{S}_{n_{2}+1}^{\prime}$.

In view of $(\mathbf{A . 7}),(\mathbf{A . 8})$ and $(\mathbf{A . 9})$ we conclude that the map $\mathcal{F}_{i}$ induces a unitary equivalence of representations

$$
\mathrm{B}_{i}: \mathcal{A}_{i} \rightarrow \mathcal{A}_{i-1} \text {. }
$$


Note that if $\varphi$ is a smooth vector in $\mathcal{A}_{i}$ then for any $g \in \mathcal{Q}_{n}$ the Fourier transform of $\varphi(\cdot g)$ (a function on $\mathrm{C}_{i}$ ) is $\mathrm{B}_{i} \varphi(\cdot g)$ (as a function of $\mathrm{R}_{i-1}$ ).

We can now complete the proof of Proposition A.2. Let $\varphi \in \mathrm{I}(\mathcal{W}(\sigma))$ and let $\varphi_{n}$ : $\mathcal{Q}_{n} \rightarrow \mathcal{W}\left(\sigma_{2}\right)$ be given by

$$
\varphi_{n}(p)=\delta_{e}^{1} \varphi(w p), \quad p \in \mathcal{Q}_{n}
$$

where $\delta_{e}^{1}: \mathcal{W}\left(\sigma_{1} \otimes \sigma_{2}\right) \rightarrow \mathcal{W}\left(\sigma_{2}\right)$ denotes the evaluation map at $e$ in the first variable. We claim that $\varphi_{n} \in \mathcal{A}_{n}$ and

$$
\left(\varphi, \varphi^{\vee}\right)_{\mathrm{M}}=\left\|\varphi_{n}\right\|_{n}^{2}
$$

Indeed,

$$
\begin{aligned}
\left\|\varphi_{n}\right\|_{n}^{2} & =\int_{\mathrm{M}_{2}^{\prime} \mathrm{U}_{1}^{\prime} \mathrm{Y}_{n} \backslash \mathcal{Q}_{n}}\left\|\varphi_{n}(p)\right\|_{\mathcal{W}\left(\sigma_{2}\right)}^{2} d p \\
& =\int_{\mathrm{U}_{1}^{\prime} \backslash\left(\mathcal{Q}_{n} \cap \mathrm{M}_{1}^{\prime}\right)} \int_{\mathrm{U}^{\prime}}\left\|\varphi_{n}\left(u^{\prime} m_{1}^{\prime}\right)\right\|_{\mathcal{W}\left(\sigma_{2}\right)}^{2} d u^{\prime} d m_{1}^{\prime} \\
& =\int_{\mathrm{U}_{1} \backslash \tilde{\mathcal{Q}}_{n_{1}}} \int_{\mathrm{U}^{\prime}}\left\|\delta_{e}^{1} \varphi\left(m_{1} w u^{\prime}\right)\right\|_{\mathcal{W}\left(\sigma_{2}\right)}^{2} \delta_{\mathrm{P}^{\prime}}\left(w^{-1} m_{1} w\right) d u^{\prime} d m_{1} \\
& =\int_{\mathrm{U}_{1} \backslash \tilde{\mathcal{Q}}_{n_{1}}} \int_{\mathrm{U}^{\prime}}\left\|\delta_{m_{1}}^{1} \varphi\left(w u^{\prime}\right)\right\|_{\mathcal{W}\left(\sigma_{2}\right)}^{2} d u^{\prime} d m_{1}=\int_{\mathrm{U}^{\prime}}\left\|\varphi\left(w u^{\prime}\right)\right\|_{\mathcal{W}\left(\sigma_{1} \otimes \sigma_{2}\right)}^{2} d u^{\prime} \\
& =\left(\varphi, \varphi^{\vee}\right)_{\mathrm{M}^{\cdot}}
\end{aligned}
$$

Define $\varphi_{i} \in \mathcal{A}_{i}, i=n-1, \ldots, n_{2}$ recursively by $\varphi_{i}=\mathrm{B}_{i+1} \varphi_{i+1}$. In view of the above we have

$$
\left(\varphi, \varphi^{\vee}\right)_{\mathrm{M}}=\left\|\varphi_{n_{2}}\right\|_{n_{2}}^{2}=\int_{\mathrm{U}_{0} \backslash \mathcal{Q}_{n}}\left|\varphi_{n_{2}}(g)\right|^{2} d g .
$$

It remains to see that $\varphi_{n_{2}}$ is the Jacquet integral $\mathbf{W}(\varphi, \sigma, 0)$ of $\varphi$. To show this, observe first that $\varphi \mapsto \varphi_{n_{2}}$ is an intertwining map with respect to $\mathcal{Q}_{n}$. Since $\varphi$ is smooth, so is $\varphi_{n}$. Thus, $\varphi \mapsto \varphi_{n_{2}}(e)$ is a Whittaker functional on $\mathrm{I}(\sigma)$. By uniqueness, we necessarily have $\varphi_{n_{2}}=c \mathbf{W}(\varphi, \sigma, 0)$ for some scalar $c$. To determine $c$, it is enough to verify that $\varphi_{n_{2}}(e)=$ $\mathbf{W}(e: \varphi, \sigma, 0)$ for any $\varphi$ of our choice such that $\mathbf{W}(e: \varphi, \sigma, 0) \neq 0$. We will show this for $\varphi$ that is supported in the big cell $\mathrm{M} w \mathrm{U}^{\prime}$. Fix such $\varphi$. By descending induction on $i$ we show that for any $i>n_{2}$ and $u^{\prime} \in \mathrm{U}^{\prime}$ we have

$$
\varphi_{i}\left(u^{\prime}\right)=\int_{\mathrm{X}_{i}} \varphi_{n}\left(u u^{\prime}\right) d u=\int_{\mathrm{X}_{i}} \delta_{e}^{1} \varphi\left(w u u^{\prime}\right) d u .
$$

This follows immediately from the definition of $\mathrm{B}_{i}$ in view of the fact that in our case $\varphi_{i}$ is compactly supported on $\mathrm{U}^{\prime}$ and therefore the Fourier transform with respect to $\mathrm{C}_{i}$ is given by a convergent integral. By the same reasoning we get 


$$
\varphi_{n_{2}}(e)=\int_{\mathrm{U}^{\prime}} \delta_{e}^{\sigma_{2}} \varphi_{n}\left(u^{\prime}\right) \psi^{-1}\left(u^{\prime}\right) d u^{\prime}=\mathbf{W}(\varphi, \sigma, 0) .
$$

The proposition follows.

A.4 Finally, we reinterpret Proposition A.2 in a way suitable for the setup of Section 5.

Assume that $n=2 k$ and let $\mathrm{M}=\mathrm{M}_{(k, k)}$. Recall the automorphism $\tilde{\theta}$ of $\mathrm{G}_{k}$ given by $g \mapsto w_{0}{ }^{t} g^{-1} w_{0}$. Let $\pi \in \Pi_{\text {gen }}^{\mathrm{G}_{k}}$ and recall the equivalence $\mathfrak{y}_{\pi}: \mathcal{W}(\pi)^{\tilde{\theta}} \rightarrow \mathcal{W}^{\psi^{-1}}\left(\pi^{\vee}\right)$ of (1.3).

Embed $\mathrm{G}_{k}$ in $\mathrm{M}$ via $g \mapsto g^{J}=\left(g, g^{\tilde{\theta}}\right)$. Recall the mirabolic subgroup $\mathcal{Q}_{k}$ of $\mathrm{G}_{k}$. For $\varrho=\pi \otimes \pi \in \Pi_{\text {gen }}^{\mathrm{M}}$ let

$$
\beta_{0}^{\varrho}(\mathrm{W})=\mathrm{L}\left(k, 1_{\mathrm{F}^{*}}\right)\left(\int_{\mathrm{U}_{0}^{J} \backslash \mathcal{Q}_{k}^{J}} \mathrm{~W}(p)|\operatorname{det} p|^{s} d p\right)_{s=0}, \quad \mathrm{~W} \in \mathcal{W}(\varrho) .
$$

Then $0 \not \equiv \beta_{0}^{\varrho} \in \operatorname{Hom}_{\mathrm{G}_{k}^{J}}(\mathcal{W}(\varrho), \mathbf{C})$ and $\beta_{0}^{\varrho}\left(\mathrm{W}_{1} \otimes \mathrm{W}_{2}\right)=\left[\mathrm{W}_{1}, \mathfrak{y}_{\pi}\left(\mathrm{W}_{2}\right)\right]_{\pi}$ for any $\mathrm{W}_{1}, \mathrm{~W}_{2} \in$ $\mathcal{W}(\pi)$.

Now let $\kappa=\left(n_{1}, \ldots, n_{s}\right)$ be a composition of $k$ and let $\mathrm{L}=\mathrm{M}_{(\kappa, \overleftarrow{\kappa})}$. (We recall that $\left.\overleftarrow{\kappa}=\left(n_{s}, \ldots, n_{1}\right).\right)$ Let $\varrho \in \Pi_{\text {gen }}^{\mathrm{L}}$ be of the form

$$
\varrho=\sigma \otimes \overleftarrow{\sigma}
$$

where $\sigma=\sigma_{1} \otimes \cdots \otimes \sigma_{s} \in \Pi_{\text {gen }}^{\mathrm{M}_{\kappa}}$ and $\overleftarrow{\sigma}=\sigma_{s} \otimes \cdots \otimes \sigma_{1} \in \Pi_{\text {gen }}^{\mathrm{M}_{\overleftarrow{\kappa}}}$. Define

$$
\beta_{0}^{\varrho}(\mathrm{W})=\left[\prod_{i=1}^{s} \mathrm{~L}\left(n_{i}, 1_{\mathrm{F}^{*}}\right)\right]\left(\int_{\left(\mathrm{U}_{0} \cap \mathrm{M}_{\kappa}\right)^{\jmath} \backslash \mathcal{Q}_{\kappa}^{J}} \mathrm{~W}(p)|\operatorname{det} p|^{z} d p\right)_{z=0}, \quad \mathrm{~W} \in \mathcal{W}(\varrho)
$$

where $\mathcal{Q}_{\kappa}$ is the mirabolic subgroup of $\mathrm{M}_{\kappa}$ (i.e., the product of the mirabolic subgroups of $\left.\mathrm{G}_{n_{i}}, i=1, \ldots, s\right)$. From $(\mathbf{1 . 5})$ and Proposition A.2 we obtain

Corollary A.5. - Suppose that $v=\mathrm{I}_{\mathrm{L}}^{\mathrm{M}}(\varrho, \lambda) \in \Pi_{\text {gen }}^{\mathrm{M}}$ with $\varrho$ as above and $w_{0} \lambda=\lambda$ (so that $\lambda$ is of the form $(\mu, \overleftarrow{\mu})$ ). Then $v$ is $\mathrm{G}_{k}^{J}$-distinguished and

$$
\beta_{0}^{v}\left(\mathbf{W}_{\mathrm{L}}^{\mathrm{M}}(\varphi, \varrho, \lambda)\right)=\int_{\mathrm{P}_{k}^{J} \backslash \mathrm{G}_{k}^{J}} \beta_{0}^{\varrho}[\varphi(g)] d g, \quad \varphi \in \mathrm{I}_{\mathrm{L}}^{\mathrm{M}}(\mathcal{W}(\varrho)) .
$$

\section{Appendix B: Distinguished representations in the Archimedean case, by Avraham Aizenbud and Erez Lapid}

In this appendix we consider representations of $\mathrm{G}=\mathrm{GL}(n, \mathbf{C})$ and a unitary group $\mathrm{G}^{x}=\mathrm{U}(p, q) \subseteq \mathrm{G}$ defined with respect to a Hermitian form $x$ with signature $(p, q)$. Recall that we denote complex conjugation by $\tau$, the diagonal torus of $\mathrm{G}$ by $\mathrm{M}_{0}$ and the 
upper-triangular Borel subgroup by $\mathrm{P}_{0}$. For a character $\chi$ of $\mathrm{M}_{0}$ we denote by $\mathrm{I}(\chi)$ the representation induced from the character $\chi$ on $\mathrm{P}_{0}$.

Let $\mathrm{W}_{2}$ be the set of involutions in $\mathrm{W}$. Any $w \in \mathrm{W}_{2}$ can be written as a product of $g_{w}$ disjoint transpositions where the number of fixed points of $w$ is $f_{w}=n-2 g_{w}$. Set $\mathfrak{m}(w)=\left(\begin{array}{c}f_{w} \\ q-g_{w}\end{array}\right)=\left(\begin{array}{c}f_{w} \\ p-g_{w}\end{array}\right)\left(=0\right.$ if $\left.g_{w}>\mathfrak{w}(x)=\min (p, q)\right)$.

In this appendix we will prove the following result.

Theorem B.1. - Let $\chi=\left(\chi_{1}, \ldots, \chi_{n}\right)$ be a character of $\mathrm{M}_{0}$ such that $|\chi(t)|=$ $\left|t_{1}\right|^{\lambda_{1}} \cdots\left|t_{n}\right|^{\lambda_{n}}$ with $\lambda_{1} \geq \cdots \geq \lambda_{n}$. Then

$$
\operatorname{dim} \operatorname{Hom}_{\mathrm{G}^{x}}(\mathrm{I}(\chi), \mathbf{C}) \leq \sum_{w \in \mathrm{W}_{2}: w \chi=\chi^{\tau}} \mathfrak{m}(w)
$$

Thus, if $\pi$ is the Langlands quotient of $\mathrm{I}(\chi)$ then

$$
\operatorname{dim} \operatorname{Hom}_{\mathrm{G}^{x}}(\pi, \mathbf{G}) \leq \sum_{w \in \mathrm{W}_{2}: w \chi=\chi^{\tau}} \mathfrak{m}(w) .
$$

In particular, if $\pi$ is $\mathrm{G}^{x}$-distinguished then there exists $w \in \mathrm{W}_{2}$ with $g_{w} \leq \mathfrak{w}(x)$ such that $w \chi=\chi^{\tau}$. Hence, $\pi$ is $\tau$-invariant and $\tilde{\mathfrak{w}}(\pi) \leq \mathfrak{w}(x)$.

For $w \in \mathrm{W}_{2}$ set $\mathrm{I}_{w}=\{(i, j): i>j, w(i)<w(j)\}$ and define for any function $\kappa$ : $\mathrm{I}_{w} \rightarrow \mathbf{Z}_{\geq 0}$ a character of $\mathrm{M}_{0}$ by

$$
\alpha_{\kappa}\left(\operatorname{diag}\left(t_{1}, \ldots, t_{n}\right)\right)=\prod_{(i, j) \in \mathrm{I}_{w}}\left[\frac{t_{i}}{t_{j}}\right]^{\kappa(i, j)} .
$$

Let

$$
\mathrm{S}_{w}(\chi)=\left\{\kappa: \mathrm{I}_{w} \rightarrow \mathbf{Z}_{\geq 0} \mid \chi^{\tau} w \chi^{-1}=\alpha_{\kappa}^{\tau} w \alpha_{\kappa}^{-1}\right\} .
$$

Note that if $\chi$ satisfies the assumption of Theorem B.1 then

$$
\mathrm{S}_{w}(\chi)= \begin{cases}\{\kappa \equiv 0\} & \text { if } w \chi=\chi^{\tau} \\ \varnothing & \text { otherwise. }\end{cases}
$$

Thus, Theorem B.1 would follow from the following Proposition which will be proved at the end of the appendix.

Proposition B.2. - Let $\chi$ be a character of $\mathrm{M}_{0}$. Then

$$
\operatorname{dim} \operatorname{Hom}_{\mathrm{G}^{x}}(\mathrm{I}(\chi), \mathbf{G}) \leq \sum_{w \in \mathrm{W}_{2}} \mathfrak{m}(w)\left|\mathrm{S}_{w}(\chi)\right|
$$


We will prove the Proposition by representing the $G^{x}$-invariant linear forms on $\mathrm{I}(\chi)$ as equivariant distributions on the Schwartz space of $\mathrm{G} / \mathrm{G}^{x}$ and using the analysis of equivariant distributions developed in [AG08].

Henceforth, we will use the following notational conventions. For now, $G$ is an arbitrary group.

- For any $\mathrm{G}$-set $\mathrm{X}$ and a point $x \in \mathrm{X}$, we denote by $\mathrm{G}(x)$ the $\mathrm{G}$-orbit of $x$ and by $\mathrm{G}^{x}$ the stabilizer of $x$.

- For any representation of $\mathrm{G}$ on a vector space $\mathrm{V}$, we denote by $\mathrm{V}^{\mathrm{G}}$ the subspace of $\mathrm{G}$-invariant vectors in $\mathrm{V}$. For a character $\chi$ of $\mathrm{G}$, we denote by $\mathrm{V}^{\mathrm{G}, \chi}$ the subspace of $(\mathrm{G}, \chi)$-equivariant vectors in $\mathrm{V}$.

- Given manifolds $\mathrm{L} \subseteq \mathrm{M}$, we denote by $\mathrm{N}_{\mathrm{L}}^{\mathrm{M}}:=\left(\left.\mathrm{T}_{\mathrm{M}}\right|_{\mathrm{L}}\right) / \mathrm{T}_{\mathrm{L}}$ the normal bundle to $\mathrm{L}$ in $\mathrm{M}$ and by $\mathrm{CN}_{\mathrm{L}}^{\mathrm{M}}:=\left(\mathrm{N}_{\mathrm{L}}^{\mathrm{M}}\right)^{*}$ the conormal bundle. For any point $y \in \mathrm{L}$, we denote by $\mathrm{N}_{\mathrm{L}, y}^{\mathrm{M}}$ the normal space to $\mathrm{L}$ in $\mathrm{M}$ at the point $y$ and by $\mathrm{CN}_{\mathrm{L}, y}^{\mathrm{M}}$ the conormal space.

- The symmetric algebra of a vector space $\mathrm{V}$ will be denoted by $\operatorname{Sym}(\mathrm{V})=$ $\bigoplus_{k \geq 0} \operatorname{Sym}^{k}(\mathrm{~V})$.

We will use the theory of Schwartz functions and distributions on Nash manifolds as developed in [AG08] generalizing the usual notions for $\mathbf{R}^{n}{ }^{13}$

We denote the Fréchet space of Schwartz functions on a Nash manifold X by $\mathcal{S}(\mathrm{X})$ and the dual space of Schwartz distributions by $\mathcal{S}^{*}(\mathrm{X}):=\mathcal{S}(\mathrm{X})^{*}$. For a closed subset $\mathrm{Z}$ of a smooth manifold X we set $\mathcal{S}_{\mathrm{X}}^{*}(\mathrm{Z}):=\left\{\xi \in \mathcal{S}^{*}(\mathrm{X}): \operatorname{supp}(\xi) \subseteq \mathrm{Z}\right\}$. More generally, for a locally closed subset $\mathrm{Y} \subseteq \mathrm{X}$ we set $\mathcal{S}_{\mathrm{X}}^{*}(\mathrm{Y}):=\mathcal{S}_{\mathrm{X} \backslash(\overline{\mathrm{Y}} \backslash \mathrm{Y})}^{*}(\mathrm{Y})$.

If $\mathrm{U}$ is an open Nash submanifold of $\mathrm{X}$ then we have the following exact sequence

$$
0 \rightarrow \mathcal{S}_{\mathrm{X}}^{*}(\mathrm{X} \backslash \mathrm{U}) \rightarrow \mathcal{S}^{*}(\mathrm{X}) \rightarrow \mathcal{S}^{*}(\mathrm{U}) \rightarrow 0
$$

For any Nash vector bundle $\mathrm{E}$ over $\mathrm{X}$ we denote by $\mathcal{S}(\mathrm{X}, \mathrm{E})$ the space of Schwartz sections of $\mathrm{E}$ and by $\mathcal{S}^{*}(\mathrm{X}, \mathrm{E})$ its dual space.

We denote by $\mathrm{D}_{\mathrm{X}}$ the bundle of densities over $\mathrm{X}$ [AG08, A.1.1] and by $\mathcal{G}(\mathrm{X}):=$ $\mathcal{S}^{*}\left(\mathrm{X}, \mathrm{D}_{\mathrm{X}}\right)$ the space of generalized functions on $\mathrm{X}$. More generally we set $\mathcal{G}(\mathrm{X}, \mathrm{E}):=$ $\mathcal{S}^{*}\left(\mathrm{X}, \mathrm{E}^{*} \otimes \mathrm{D}_{\mathrm{X}}\right)$ for any Nash vector bundle $\mathrm{E}$ over $\mathrm{X}$. Note that $\mathcal{S}(\mathrm{X}, \mathrm{E})$ is naturally embedded into $\mathcal{G}(\mathrm{X}, \mathrm{E})$ but not into $\mathcal{S}^{*}(\mathrm{X}, \mathrm{E})$. For any locally closed subset $\mathrm{Y}$ of $\mathrm{X}$, the spaces $\mathcal{S}_{\mathrm{X}}^{*}(\mathrm{Y}, \mathrm{E}), \mathcal{G}_{\mathrm{X}}(\mathrm{Y}, \mathrm{E})$ and $\mathcal{G}_{\mathrm{X}}(\mathrm{Y})$ are similarly defined.

Suppose that a group $\mathrm{G}$ acts on a Nash manifold $\mathrm{X}$. Then $\mathrm{G}$ naturally acts on $\mathcal{S}(\mathrm{X})$ and $\mathcal{S}^{*}(\mathrm{X})$, and $\mathrm{T}_{\mathrm{X}}$ has a natural G-equivariant structure. Therefore all the standard bundles constructed from $\mathrm{T}_{\mathrm{X}}$, such as $\mathrm{D}_{\mathrm{X}}$, also have a $\mathrm{G}$-equivariant structure. This gives rise to an action of $\mathrm{G}$ on $\mathcal{S}\left(\mathrm{X}, \mathrm{D}_{\mathrm{X}}\right)$ and the dual action on $\mathcal{G}(\mathrm{X})$. Note that the G-action on $\mathcal{G}(\mathrm{X})$ extends the action on $\mathcal{S}(\mathrm{X})$ and similarly the action on $\mathcal{S}^{*}(\mathrm{X})$ extends the action on $\mathcal{S}\left(\mathrm{X}, \mathrm{D}_{\mathrm{X}}\right)$.

We will use some standard facts about equivariant distributions.

\footnotetext{
${ }^{13}$ In the present context we will only apply it to smooth real algebraic manifolds.
} 
Proposition B.3. - Let a Nash group $\mathrm{G}$ act on a Nash manifold $\mathrm{X}$. Let $\mathrm{Z} \subseteq \mathrm{X}$ be a closed $\mathrm{G}$-invariant subset with a $\mathrm{G}$-invariant stratification $\mathrm{Z}=\bigcup_{i=0}^{l} \mathrm{Z}_{i}$. Let $\chi$ be a character of $\mathrm{G}$. Then

$$
\operatorname{dim}\left(\mathcal{S}_{\mathrm{X}}^{*}(\mathrm{Z})^{\mathrm{G}, \chi}\right) \leq \sum_{i=0}^{l} \sum_{k=0}^{\infty} \operatorname{dim}\left(\mathcal{S}^{*}\left(\mathrm{Z}_{i}, \operatorname{Sym}^{k}\left(\mathrm{CN}_{\mathrm{Z}_{i}}^{\mathrm{X}}\right)\right)^{\mathrm{G}, \chi}\right)
$$

The proof is the same as in [AGS08, Corollary B.2.4].

Let $\phi: \mathrm{M} \rightarrow \mathrm{N}$ be a Nash submersion of Nash manifolds. Let $\mathrm{E}$ be a bundle on $\mathrm{N}$. We denote by $\phi^{*}: \mathcal{G}(\mathrm{N}, \mathrm{E}) \rightarrow \mathcal{G}\left(\mathrm{M}, \phi^{*}(\mathrm{E})\right)$ the pull back of generalized functions [AG09a, Notation B.2.5].

Proposition B.4. - Let $\mathrm{M}$ be a Nash manifold. Let $\mathrm{K}$ be a Nash group. Let $\mathrm{E} \rightarrow \mathrm{M}$ be a Nash bundle. Consider the standard projection $p: \mathrm{K} \times \mathrm{M} \rightarrow \mathrm{M}$. Then the map $p^{*}: \mathcal{G}(\mathrm{M}, \mathrm{E}) \rightarrow$ $\mathcal{G}\left(\mathrm{M} \times \mathrm{K}, p^{*} \mathrm{E}\right)^{\mathrm{K}}$ is an isomorphism.

For a proof see [AG09a, Proposition B.3.1].

Corollary B.5. - Let $\mathrm{G}$ be a real algebraic group and $\mathrm{H} \subseteq \mathrm{G}$ a closed subgroup. Then $\mathcal{G}(\mathrm{G})^{\mathrm{H}} \cong \mathcal{G}(\mathrm{G} / \mathrm{H})$.

Proof. - By [AG10, Proposition 4.0.6] the map G $\rightarrow$ G/H is a Nash locally trivial fibration [AG10, Definition 2.4.1]. The assertion follows from Proposition B.4 by a partition of unity argument (cf. [AG08, Theorem 5.2.1]).

The following version of Frobenius reciprocity is a slight generalization of [AG09a, Theorem 2.5.7]. For the convenience of the reader we sketch a proof.

Theorem $\mathbf{B . 6}$ (Frobenius reciprocity). — Let $\mathrm{G}$ be a Nash group acting transitively on a Nash manifold $\mathrm{Z}$ and let $\varphi: \mathrm{X} \rightarrow \mathrm{Z}$ be a $\mathrm{G}$-equivariant Nash map. Fix $z \in \mathrm{Z}$ and let $\mathrm{X}_{z}$ be the fiber of $z$. Let $\chi$ be a tempered character of $\mathrm{G}\left[A G 08\right.$, Definition 5.1.1]. Then $\mathcal{S}^{*}(\mathrm{X})^{\mathrm{G}, \chi}$ is canonically isomorphic to $\mathcal{S}^{*}\left(\mathrm{X}_{z}\right)^{\mathrm{G}_{z}, \chi \delta_{\mathrm{H}}^{-1} \delta_{\mathrm{G}}}$.

Moreover, for any $\mathrm{G}$-equivariant bundle $\mathrm{E}$ on $\mathrm{X}$, the space $\mathcal{S}^{*}(\mathrm{X}, \mathrm{E})^{\mathrm{G}, \chi}$ is canonically isomorphic to $\mathcal{S}^{*}\left(\mathrm{X}_{z},\left.\mathrm{E}\right|_{\mathrm{X}_{z}}\right)^{\mathrm{G}_{z}, \chi \delta_{\mathrm{H}}^{-1} \delta_{\mathrm{G}}}$. Here $\delta_{\mathrm{G}}$ and $\delta_{\mathrm{H}}$ are the modulus characters of the groups $\mathrm{G}$ and $\mathrm{H}$.

Proof. - As in [AG09a, Theorem 2.5.7], we will prove an equivalent statement for generalized functions. Namely, we will construct canonical isomorphisms HC: $\mathcal{G}(\mathrm{X}, \mathrm{E})^{\mathrm{G}, \chi} \rightarrow \mathcal{G}\left(\mathrm{X}_{z},\left.\mathrm{E}\right|_{\mathrm{X}_{z}}\right)^{\mathrm{G}_{z}, \chi}$ and Fr $: \mathcal{G}\left(\mathrm{X}_{z},\left.\mathrm{E}\right|_{\mathrm{X}_{z}}\right)^{\mathrm{G} z, \chi} \rightarrow \mathcal{G}(\mathrm{X}, \mathrm{E})^{\mathrm{G}, \chi}$.

Consider the natural submersion $a: \mathrm{G} \times \mathrm{X}_{z} \rightarrow \mathrm{X}$ and the projection $p: \mathrm{G} \times \mathrm{X}_{z} \rightarrow$ $\mathrm{X}_{z}$. Note that the equivariant structure of $\mathrm{E}$ gives us an identification $\phi: a^{*}(\mathrm{E}) \rightarrow$ $p^{*}\left(\left.\mathrm{E}\right|_{\mathrm{X}_{z}}\right)$. Consider the tempered function $f$ on $\mathrm{G} \times \mathrm{X}_{z}$ given by $f(g, x)=\chi^{-1}(g)$. Define the map $a^{*, \chi}: \mathcal{G}(\mathrm{X}, \mathrm{E})^{\mathrm{G}, \chi} \rightarrow \mathcal{G}\left(\mathrm{G} \times \mathrm{X}_{z}, p^{*}\left(\left.\mathrm{E}\right|_{\mathrm{X}_{z}}\right)\right)^{\mathrm{G}}$ by $a^{*, \chi}(\xi)=f \phi\left(a^{*}(\xi)\right)$. Here, the action of $\mathrm{G}$ on $\mathrm{G} \times \mathrm{X}_{z}$ is on the first coordinate. On the other hand, By Proposi- 
tion B.4 we have $\mathcal{G}\left(\mathrm{G} \times \mathrm{X}_{z}, p^{*}\left(\left.\mathrm{E}\right|_{\mathrm{X}_{z}}\right)\right)^{\mathrm{G}} \cong \mathcal{G}\left(\mathrm{X}_{z},\left.\mathrm{E}\right|_{\mathrm{X}_{z}}\right)$. Together, this gives the required map HC. A similar modification to the construction of Fr in [AG09a, Theorem 2.5.7] gives rise to Fr in our context.

Proof of Proposition B.2. - Let $\mathrm{G}=\mathrm{GL}_{n}(\mathbf{C})$ and $\mathrm{H}=\mathrm{U}(p, q)$. Note that after identifying $\mathrm{D}_{\mathrm{G}}$ and $\mathrm{D}_{\mathrm{G} / \mathrm{H}}$ with the trivial bundle (in a G-equivariant way) we have

$$
\mathrm{I}(\chi)^{*}=\mathcal{G}(\mathrm{G})^{\mathrm{P}_{0}, \chi \delta_{0}^{-1 / 2}}=\mathcal{S}^{*}(\mathrm{G})^{\mathrm{P}_{0}, \chi \delta_{0}^{-\frac{1}{2}}}
$$

where $\mathrm{P}_{0}$ acts on generalized functions on the left. Therefore

$$
\operatorname{Hom}_{\mathrm{H}}(\mathrm{I}(\chi), \mathbf{G})=\mathcal{G}(\mathrm{G} / \mathrm{H})^{\mathrm{P}_{0}, \chi \delta_{0}^{-1 / 2}}=\mathcal{S}^{*}(\mathrm{G} / \mathrm{H})^{\mathrm{P}_{0}, \chi \delta_{0}^{-\frac{1}{2}}} .
$$

We can stratify $\mathrm{G} / \mathrm{H}$ by $\mathrm{P}_{0}$-orbits. By Remark 6.3 any such orbit contains a unique element $x$ of the form $x=w a$ where $w \in \mathrm{W}_{2}$ and $a \in \mathrm{M}_{0}$ is such that $a_{i}=1$ if $w(i) \neq i$ and $a_{i}= \pm 1$ otherwise. The number of $\mathrm{P}_{0}$-orbits on $\mathrm{G} / \mathrm{H}$ above a given $w \in \mathrm{W}_{2}$ is precisely $\mathfrak{m}(w)$ and moreover,

$$
\mathbf{M}_{0}^{x}=\mathbf{M}_{0}^{w}=\left\{t \in \mathrm{M}_{0}: t w t^{\tau} w=1\right\}=\left\{t w\left(t^{-1}\right)^{\tau} w: t \in \mathrm{M}_{0}\right\} .
$$

Using Proposition B.3, it suffices to show that for any $w$ and $a$ as above we have

$$
\sum_{k=0}^{\infty} \operatorname{dim}\left(\mathcal{S}^{*}\left(\mathrm{P}_{0}(x), \operatorname{Sym}^{k}\left(\mathrm{CN}_{\mathrm{P}_{0}(x)}^{\mathrm{X}}\right)\right)^{\mathrm{P}_{0}, \chi \delta_{0}^{-1 / 2}}\right) \leq\left|\mathrm{S}_{w}(\chi)\right| .
$$

By Theorem B.6 and the relation $\left.\delta_{0}^{1 / 2}\right|_{\mathrm{P}_{0}^{x}}=\delta_{\mathrm{P}_{0}^{x}}[$ LR03, Proposition 4.3.2] we get

$$
\begin{aligned}
\mathcal{S}^{*}\left(\mathrm{P}_{0}(x), \operatorname{Sym}^{k}\left(\mathrm{CN}_{\mathrm{P}_{0}(x)}^{\mathrm{X}}\right)\right)^{\mathrm{P}_{0}, \chi \delta_{0}^{-1 / 2}} & =\mathcal{S}^{*}\left(\{x\}, \operatorname{Sym}^{k}\left(\mathrm{CN}_{\mathrm{P}_{0}(x), x}^{\mathrm{X}}\right)\right)^{\mathrm{P}_{0}, \chi \delta_{0}^{-1 / 2} \delta_{\mathrm{P}_{0}^{\delta}}^{-1} \delta_{0}} \\
& =\mathcal{S}^{*}\left(\{x\}, \operatorname{Sym}^{k}\left(\mathrm{CN}_{\mathrm{P}_{0}(x), x}^{\mathrm{X}}\right)\right)^{\mathrm{P}_{0}, \chi} \\
& =\left(\operatorname{Sym}^{k}\left(\mathrm{~N}_{\mathrm{P}_{0}(x), x}^{\mathrm{X}}\right) \otimes_{\mathbf{R}} \mathbf{C}\right)^{\mathrm{P}_{0}, \chi} .
\end{aligned}
$$

We reduce to showing that

$$
\operatorname{dim}\left(\operatorname{Sym}\left(\mathrm{N}_{\mathrm{P}_{0}(x), x}^{\mathrm{G} / \mathrm{H}}\right) \otimes_{\mathbf{R}} \mathbf{C}\right)^{\mathrm{P}_{0}^{x}, \chi} \leq\left|\mathrm{S}_{w}(\chi)\right| .
$$

To that end, it suffices to show that

$$
\operatorname{Sym}\left(\mathrm{N}_{\mathrm{P}_{0}(x), x}^{\mathrm{G} / \mathrm{H}}\right) \otimes_{\mathbf{R}} \mathbf{G}=\bigoplus_{\kappa: \mathrm{I}_{w} \rightarrow \mathbf{Z}_{\geq 0}} \alpha_{\kappa}
$$

as a representation of $\mathbf{M}_{0}^{x}$. Indeed, by $(\mathbf{B . 1})$ we have

$$
\left.\alpha_{\kappa}\right|_{\mathrm{M}_{0}^{x}}=\left.\chi\right|_{\mathrm{M}_{0}^{x}} \quad \Longleftrightarrow \quad \kappa \in \mathrm{S}_{w}(\chi)
$$


and hence it would follow that

$$
\operatorname{dim}\left(\operatorname{Sym}\left(\mathrm{N}_{\mathrm{P}_{0}(x), x}^{\mathrm{G} / \mathrm{H}}\right) \otimes_{\mathbf{R}} \mathbf{C}\right)^{\mathrm{P}_{0}^{x}, \chi} \leq \operatorname{dim}\left(\operatorname{Sym}\left(\mathrm{N}_{\mathrm{P}_{0}(x), x}^{\mathrm{G} / \mathrm{H}}\right) \otimes_{\mathbf{R}} \mathbf{G}\right)^{\mathrm{M}_{0}^{x}, \chi} \leq\left|\mathrm{S}_{w}(\chi)\right|
$$

as required.

It remains to show $(\mathbf{B . 2})$. We will deduce it by showing that

$$
\mathrm{N}_{\mathrm{P}_{0}(x), x}^{\mathrm{G} / \mathrm{H}} \otimes_{\mathbf{R}} \mathbf{G} \cong \bigoplus_{\imath \in \mathrm{I}_{w}} \alpha_{\delta_{l}}
$$

as a representation of $\mathrm{M}_{0}^{x}$ where $\delta_{l}$ is defined by $\delta_{l}(\mathrm{~J})=\delta_{l, \jmath}$.

We have

$$
\mathrm{N}_{\mathrm{P}_{0}(x), x}=\operatorname{Herm} / \operatorname{Im}(\phi)
$$

where Herm is the space of $n \times n$ Hermitian matrices and $\phi: \operatorname{Lie}\left(\mathrm{P}_{0}\right) \rightarrow$ Herm is defined by $\phi(b)=b w a+w a^{t} b^{\tau}$.

It is easy to see that

$$
\begin{aligned}
\operatorname{Im}(\phi) & =\operatorname{Span}_{\mathbf{G}}\left(\left\{e_{i, w(j)}, e_{w(j), i}: j \geq i\right\}\right) \cap \text { Herm } \\
& =\operatorname{Span}_{\mathbf{G}}\left(\left\{e_{i, j}, e_{j, i}: w(j) \geq i\right\}\right) \cap \text { Herm } \\
& =\operatorname{Span}_{\mathbf{G}}\left(\left\{e_{i, j}: w(j) \geq i \text { or } w(i) \geq j\right\}\right) \cap \text { Herm }
\end{aligned}
$$

where $e_{i, j}$ is the standard basis for $n \times n$ matrices. Therefore

$$
\begin{aligned}
\mathrm{N}_{\mathrm{P}_{0}(x), x} \cong & \operatorname{Span}_{\mathbf{G}}\left(\left\{e_{i, j}: i>w(j), j>w(i)\right\}\right) \cap \text { Herm } \\
= & \operatorname{Span}_{\mathbf{G}}\left(\left\{e_{i, w(j)}: i>j, w(j)>w(i)\right\}\right) \cap \text { Herm } \\
= & \operatorname{Span}_{\mathbf{C}}\left(\left\{e_{i, w(j)}:(i, j) \in \mathrm{I}_{w}\right\}\right) \cap \text { Herm } \\
& \cong \bigoplus_{\left\{(i, j) \in \mathrm{I}_{w}: i=w(j)\right\}} \operatorname{Span}_{\mathbf{R}}\left(e_{i, w(j)}\right) \\
& \bigoplus \bigoplus_{\left\{(i, j) \in \mathrm{I}_{w}: i<w(j)\right\}} \operatorname{Span}_{\mathbf{R}}\left(e_{i, w(j)}+e_{w(j), i}, \sqrt{-1}\left(e_{i, w(j)}-e_{w(j), i}\right)\right) .
\end{aligned}
$$

By $(\mathbf{B . 1})$, the action of $\mathbf{M}_{0}^{x}$ on $e_{i, w(j)}$ is given by $\alpha_{\delta_{(i, j)}}=t_{i} / t_{j}$. Thus, as a representation of $\mathrm{M}_{0}^{x}$ we have

$$
\begin{aligned}
\mathrm{N}_{\mathrm{P}_{0}(x), x} \otimes_{\mathbf{R}} \mathbf{G} & \cong \bigoplus_{\left\{(i, j) \in \mathrm{I}_{w}, i=w(j)\right\}} \alpha_{\delta_{(i, j)}} \oplus \bigoplus_{\left\{(i, j) \in \mathrm{I}_{w}, i<w(j)\right\}}\left(\alpha_{\delta_{(i, j)}} \oplus \alpha_{\delta_{(i, j)}^{\tau}}^{\tau}\right) \\
& =\bigoplus_{\left\{(i, j) \in \mathrm{I}_{w}, i=w(j)\right\}} \alpha_{\delta_{(i, j)}} \oplus \bigoplus_{\left\{(i, j) \in \mathrm{I}_{w}, i<w(j)\right\}}\left(\alpha_{\delta_{(i, j)}} \oplus \alpha_{\delta_{(w(j) w(i))}}\right)
\end{aligned}
$$




$$
\begin{gathered}
=\bigoplus_{\left\{(i, j) \in \mathrm{I}_{w}, i=w(j)\right\}} \alpha_{\delta_{(i, j)}} \oplus \bigoplus_{\left\{(i, j) \in \mathrm{I}_{w}, i<w(j)\right\}} \alpha_{\delta_{(i, j)}} \\
\oplus \bigoplus_{\left\{(i, j) \in \mathrm{I}_{w}, i>w(j)\right\}} \alpha_{\delta_{(i, j)}}=\bigoplus_{i \in \mathrm{I}_{w}} \alpha_{\delta_{l}}
\end{gathered}
$$

as required.

Finally, we go back to the definition of the open periods in Section 4.

Theorem B.7. - For any $\lambda \in \mathfrak{a}_{\mathrm{M}_{0}, \mathbf{G}}^{*}$ with $\operatorname{Re} \lambda_{1}>\cdots>\operatorname{Re} \lambda_{n}$ the map $\alpha \mapsto \mathrm{J}(\alpha, \lambda)$ is holomorphic and defines an isomorphism $\left.\mathcal{E}_{\mathrm{M}_{0}}\left(\mathrm{X}^{\mathrm{M}_{0}}, 1_{\mathrm{M}_{0}}^{*}\right) \rightarrow \mathcal{E}_{\mathrm{G}}\left(\mathrm{X}, \mathrm{I}_{\left(\mathrm{M}_{0}\right.}, \lambda\right)^{*}\right)$.

Proof. - Let $\chi_{\lambda}$ be the character $t \mapsto\left|t_{1}\right|^{\lambda_{1}} \ldots\left|t_{n}\right|^{\lambda_{n}}$ of $\mathrm{M}_{0}$. The argument above shows that for $\lambda$ as in the statement, unitary periods on $\left.\mathrm{I}_{\left(\mathrm{M}_{0}\right.}, \lambda\right)$ are supported on open orbits, in the sense that for any $x$ outside the open orbits we have $\left(\operatorname{Sym}\left(\mathrm{N}_{\mathrm{P}_{0}(x), x}^{\mathrm{G} / \mathrm{H}}\right) \otimes_{\mathbf{R}}\right.$ C) ${ }^{\mathrm{P}_{0}^{x}, \chi_{\lambda}}=0$. We can now argue exactly as in the proof of Lemma 6.7.

\section{Appendix G: The relative trace formula, by Erez Lapid and Omer Offen}

For $f^{\prime} \in \mathcal{S}\left(\mathrm{G}_{\mathbf{A}}^{\prime}\right)$ and $\Phi \in \mathcal{S}\left(\mathrm{X}_{\mathbf{A}}\right)$ let

$$
\mathrm{K}_{f^{\prime}}^{\prime}(x, y)=\sum_{\gamma \in \mathrm{G}^{\prime}} f^{\prime}\left(x^{-1} \gamma y\right), \quad x, y \in \mathrm{G}_{\mathbf{A}}^{\prime}
$$

and

$$
\mathrm{K}_{\Phi}(g)=\sum_{x \in \mathrm{X}} \Phi(x \bullet g), \quad g \in \mathrm{G}_{\mathbf{A}} .
$$

Define the relative trace formula

$$
\operatorname{RTF}(\Phi)=\int_{\mathrm{U}_{0} \backslash \mathrm{U}_{0, \mathbf{A}}} \mathrm{K}_{\Phi}(u) \psi_{0}(u) d u
$$

and the Kuznetsov trace formula

$$
\operatorname{KTF}\left(f^{\prime}\right)=\int_{\mathrm{U}_{0}^{\prime} \backslash \mathrm{U}_{0, \mathbf{A}}^{\prime}} \int_{\mathrm{U}_{0}^{\prime} \backslash \mathrm{U}_{0, \mathbf{A}}^{\prime}} \mathrm{K}_{f^{\prime}}\left({ }^{t} u_{1}^{-1}, u_{2}\right) \psi_{0}^{\prime}\left(u_{1} u_{2}\right) d u_{1} d u_{2} .
$$

Expanding the geometric sides according to double cosets and applying [Jac03a, Théorème 1.1] for the non-Archimedean case and [AG] for the Archimedean case, we see that for $\Phi$ and $f^{\prime}$ matching we have

$$
\operatorname{RTF}(\Phi)=\operatorname{KTF}\left(f^{\prime}\right)
$$


Using the spectral expansion for the kernel (cf. [Art78, §4]) and the computation of the Whittaker coefficient of Eisenstein series, the spectral decomposition of $\mathrm{KTF}\left(f^{\prime}\right)$ is given by

$$
\operatorname{KTF}\left(f^{\prime}\right)=\sum_{\left(\mathrm{M}^{\prime}, \pi^{\prime}\right) \in \mathcal{X}^{\prime}} \int_{\mathrm{ia}_{\mathrm{M}}^{*}} \mathbf{B}\left(f^{\prime}: \pi^{\prime}, \lambda^{\prime}\right) d \lambda^{\prime}
$$

where $\mathcal{X}^{\prime}$ is the set of pairs $\left(\mathrm{M}^{\prime}, \pi^{\prime}\right), \pi^{\prime} \in \mathcal{C}^{\mathrm{M}^{\prime}}$ up to conjugation and we recall that $\mathbf{B}\left(f^{\prime}: \pi^{\prime}, \lambda^{\prime}\right)$ was defined in $(\mathbf{1 1 . 1})$.

The spectral expansion of $\operatorname{RTF}(\Phi)$ was considered in [Lap06]. Only pairs (L, $\pi$ ), $\pi \in \mathcal{C}^{\mathrm{L}}$ which are conjugate to $\left(\mathrm{L}, \pi^{\tau}\right)$ contribute. We can conjugate such $(\mathrm{L}, \pi)$ to have the form

$$
\pi=\varrho_{1} \otimes \cdots \otimes \varrho_{s} \otimes \sigma_{1} \otimes \cdots \otimes \sigma_{r} \otimes \varrho_{s}^{\tau} \otimes \cdots \otimes \varrho_{1}^{\tau}
$$

where $\varrho_{i} \in \mathcal{C}^{\mathrm{GL}_{n_{i}}, \neg \tau}, i=1, \ldots, s$ and $\sigma_{j} \in \mathcal{C}^{\mathrm{GL}_{m_{j}}, \tau}, j=1, \ldots, r$. Denote by $\mathcal{X}^{\tau}$ the collection of all such cuspidal data $(\mathrm{L}, \pi)$. Let $\mathrm{P}=\mathrm{MU}$ be the parabolic of type $\left(n_{1}, \ldots, n_{s}, m_{1}+\cdots+m_{r}, n_{s}, \ldots, n_{1}\right)$ and let $\mathfrak{a}_{(\mathrm{L}, \pi)}^{*}=\left(\mathfrak{a}_{\mathrm{L}}^{*}\right)^{w_{\mathrm{M}}}$, i.e., the subspace of $\mathfrak{a}_{\mathrm{L}}^{*}$ given by

$$
\left\{\left(\mu_{1}, \ldots, \mu_{s}, \lambda_{1}, \ldots, \lambda_{r}, \mu_{s}, \ldots, \mu_{1}\right): \forall i, j, \lambda_{i}, \mu_{j} \in \mathbf{R}\right\} .
$$

We define $\alpha^{(\mathrm{L}, \pi)}(\lambda) \in \mathcal{E}_{\mathrm{G}_{\mathbf{A}}}\left(\mathrm{X}_{\mathbf{A}}, \mathrm{I}(\pi, \lambda)^{*}\right), \lambda \in \mathfrak{a}_{(\mathrm{L}, \pi)}^{*}$ as follows. If $x \bullet \mathrm{G}_{\mathbf{A}} \cap w_{0} \mathrm{M}=\emptyset$ then we set $\alpha_{x}^{(\mathrm{L}, \pi)} \equiv 0$. Otherwise, suppose that $y=x \bullet g^{-1} \in \mathrm{X} \cap w_{0} \mathrm{M}$ for some $g \in \mathrm{G}_{\mathbf{A}}$ and set

$$
\alpha_{x}^{(\mathrm{L}, \pi)}(\varphi, \lambda)=\int_{\left(\mathrm{A}_{\mathrm{M}} \cap \mathrm{G}_{\mathbf{A}}^{y}\right) \mathrm{U}_{\mathbf{A}}^{y} \mathrm{M}^{y} \backslash \mathrm{G}_{\mathbf{A}}^{y}}^{*} \mathrm{E}^{\mathrm{P}}(h g, \varphi, \lambda) d h .
$$

(See [Lap06] for the meaning of the regularized integral $\int^{*}$.) This is well-defined because the M-orbit of $y$ is determined by $x$, and given $y, g$ is determined up to left multiplication by $\mathrm{G}_{\mathbf{A}}^{y}$.

In particular, if $\pi \in \mathcal{C}^{\mathrm{L}, \tau}$ then $\mathrm{M}=\mathrm{G}, \mathfrak{a}_{(\mathrm{L}, \pi)}^{*}=\mathfrak{a}_{\mathrm{L}}^{*}$ and

$$
\alpha_{x}^{(\mathrm{L}, \pi)}(\varphi, \lambda)= \begin{cases}\int_{\mathrm{G}^{y} \backslash \mathrm{G}_{\mathbf{A}}^{\nu}}^{*} \mathrm{E}(h g, \varphi, \lambda) d h & \text { if } x=y \bullet g, y \in \mathrm{X}, g \in \mathrm{G}_{\mathbf{A}} \\ 0 & x \notin \mathrm{X} \bullet \mathrm{G}_{\mathbf{A}} .\end{cases}
$$

Of course, if $\mathrm{L}=\mathrm{G}$ and $x \bullet g^{-1}=y \in \mathrm{X}$, this is just $\int_{\mathrm{G}^{y} \backslash \mathrm{G}_{\mathbf{A}}^{y}} \varphi(h g) d h$.

We set

$$
\tilde{\mathbf{B}}(\Phi: \pi, \lambda)=\tilde{\mathfrak{B}}_{\mathfrak{A}(\pi, \lambda)}^{\alpha^{(\mathrm{L}, \pi)}(\lambda), \mathbf{W}_{e}^{\psi^{-1}}(\hat{\pi},-\lambda)}(\Phi), \quad \lambda \in \mathrm{ia}_{(\mathrm{L}, \pi)}^{*} .
$$


Theorem C.1 [Lap06]. - The spectral expansion of the relative trace formula is given by

$$
\sum_{(\mathrm{L}, \pi) \in \mathcal{X}^{\tau}} c_{\mathrm{L}}^{\mathrm{M}} \int_{\mathrm{ia}_{(\mathrm{L}, \pi)}^{*}} \tilde{\mathbf{B}}(\Phi: \pi, \lambda) d \lambda
$$

where $c_{\mathrm{L}}^{\mathrm{M}}$ are certain combinatorial constants depending only on the types of $\mathrm{L}$ and $\mathrm{M}$. In particular, $c_{\mathrm{G}}^{\mathrm{G}}=1$. The integral-sum is absolutely convergent.

Remark C.2. - The formulation in [Lap06] is given in a slightly different form, but it amounts to the one given here. Note that in [ibid.] the notation suggests that $c_{\mathrm{L}}^{\mathrm{M}}$ depends on $\pi$ as well, but in fact it is clear from the proof that it does not. Finally, in [Lap06], the statement is made for compactly supported $\Phi$. However, it is easy to see that the argument extends to all $\Phi \in \mathcal{S}\left(\mathrm{X}_{\mathbf{A}}\right)$. First, for any such $\Phi$ there are still only finitely many G-orbits $x \bullet \mathrm{G}$ in $\mathrm{X}$ such that $\Phi(x \bullet g) \neq 0$ for some $g \in \mathrm{G}_{\mathbf{A}}$. Thus, just like in [ibid.] it is enough to deal with a single orbit. Everything boils down to extending the majorization of Eisenstein series given in [Lap06, Proposition 6.1] to any $f \in \mathcal{S}\left(\mathrm{G}_{\mathbf{A}}\right)$. Examining the proof, the only place where the compact support of $f$ was used is the majorization of the operator norm of $\mathrm{I}(f, \lambda)$ on the induced space (for $\operatorname{Re} \lambda$ possibly non-zero, but bounded). But in any case this is bounded by

$$
\int_{\mathrm{G}_{\mathbf{A}}} f(g)\|g\|^{\mathrm{N}} d g
$$

for some $\mathrm{N}$ (depending on $\operatorname{Re} \lambda$ ), and therefore it is still a continuous semi-norm on $\mathcal{S}\left(\mathrm{G}_{\mathbf{A}}\right)$. We note that the inequality [Lap06, (6.2)] is used only for fixed auxiliary compactly supported functions $g_{1}, g_{2}$, but never for $f$ itself.

Let us compare the two spectral expansions. Let $\mathrm{L}_{1}^{\prime}$ be a Levi subgroup of $\mathrm{G}^{\prime}$ and $\pi^{\prime} \in \mathcal{C}^{\mathrm{L}_{1}^{\prime}}$. After conjugation, we may assume that $\mathrm{L}_{1}^{\prime}$ is of type $\left(m_{1}, \ldots, m_{r}, 2 n_{1}, \ldots, 2 n_{s}\right)$ and

$$
\pi^{\prime}=\sigma_{1}^{\prime} \otimes \cdots \otimes \sigma_{r}^{\prime} \otimes \varrho_{1}^{\prime} \otimes \cdots \otimes \varrho_{s}^{\prime}
$$

where $\sigma_{i}^{\prime} \not \sigma_{i}^{\prime} \cdot \eta$ for all $i$ and $\varrho_{j}^{\prime} \simeq \varrho_{j}^{\prime} \cdot \eta$ for all $j$. Define

$$
\mathrm{bc}\left(\left(\mathrm{L}_{1}^{\prime}, \pi^{\prime}\right)\right)=(\mathrm{L}, \pi)
$$

where

$$
\pi=\varrho_{1} \otimes \cdots \otimes \varrho_{s} \otimes \mathrm{bc}\left(\sigma_{1}^{\prime}\right) \otimes \cdots \otimes \mathrm{bc}\left(\sigma_{r}^{\prime}\right) \otimes \varrho_{s}^{\tau} \otimes \cdots \otimes \varrho_{1}^{\tau}
$$

and where

$$
\operatorname{bc}\left(\varrho_{j}^{\prime}\right)=\mathrm{I}\left(\varrho_{j} \otimes \varrho_{j}^{\tau}, 0\right)
$$


i.e., $\varrho_{j}^{\prime}=\operatorname{ai}\left(\varrho_{j}\right)$ for all $j$. Note that we can identify $\mathfrak{a}_{\mathrm{bc}\left(\mathrm{L}_{1}^{\prime}, \pi^{\prime}\right)}$ with $\mathfrak{a}_{\mathrm{L}_{1}^{\prime}}^{*}$ via

$$
\lambda=\left(\mu_{1}, \ldots, \mu_{s}, \lambda_{1}, \ldots, \lambda_{r}, \mu_{s}, \ldots, \mu_{1}\right) \mapsto \lambda^{\prime}=\left(\lambda_{1}, \ldots, \lambda_{r}, \mu_{1}, \ldots, \mu_{s}\right) .
$$

Theorem C.3. - For any $(\mathrm{L}, \pi) \in \mathcal{X}^{\tau}$ and $\mathrm{M}$ as above there exists a constant $c$ depending only on the types of $\mathrm{L}$ and $\mathrm{M}$ such that for all $\lambda \in \mathrm{i} \mathfrak{a}_{(\mathrm{L}, \pi)}^{*}$ we have

$$
\tilde{\mathbf{B}}(\Phi: \pi, \lambda)=c \sum_{\left(\mathrm{L}_{1}^{\prime}, \pi^{\prime}\right): \mathrm{bc}\left(\mathrm{L}_{1}^{\prime}, \pi^{\prime}\right)=(\mathrm{L}, \pi)} \mathbf{B}\left(f^{\prime}: \pi^{\prime}, \lambda\right)
$$

for any $\Phi \longleftrightarrow f^{\prime}$. In particular, if $\pi \in \mathcal{C}^{\mathrm{L}, \tau}$ then

$$
\tilde{\mathbf{B}}(\Phi: \pi, \lambda)=c \sum_{\pi^{\prime}: \mathrm{bc}\left(\pi^{\prime}\right)=\pi} \mathbf{B}\left(f^{\prime}: \pi^{\prime}, \lambda\right) .
$$

Especially, if $\pi \in \mathcal{C}^{\mathrm{G}, \tau}$ then

$$
\tilde{\mathfrak{B}}_{\mathfrak{A}_{\pi}}^{\alpha^{\pi}, \mathbf{W}_{e}^{\psi^{-1}}}(\Phi)=\sum_{\pi^{\prime} \in \mathcal{C}^{\mathcal{G}^{\prime}}: \mathrm{bc}\left(\pi^{\prime}\right)=\pi} \mathbf{B}_{\pi^{\prime}}\left(f^{\prime}\right) .
$$

(The right-hand side consists of two summands.)

Proof. - We will use the general linear independence of characters argument [LR00, Lemma 4]. Fix $\Phi=\bigotimes \Phi_{v} \in \mathcal{S}\left(\mathrm{G}_{\mathbf{A}}\right)$ and $f^{\prime}=\bigotimes f_{v}^{\prime} \in \mathcal{S}\left(\mathrm{G}_{\mathbf{A}}^{\prime}\right)$ such that $\Phi_{v} \longleftrightarrow f_{v}^{\prime}$ for all places $v$ of $\mathrm{F}$ and a finite set $\mathrm{S}$ of places of $\mathrm{F}$ containing the Archimedean and the even ones, outside of which $\mathrm{E} / \mathrm{F}$ is unramified, $\psi_{v}^{\prime}$ has conductor $\mathcal{O}_{v}, \Phi_{v}=\mathbf{1}_{\mathrm{K}_{v} \cap \mathrm{X}_{v}}$ and $f_{v}^{\prime}=\mathbf{1}_{\mathrm{K}_{v}^{\prime}}$. Let $f^{\mathrm{S}}$ be in the Hecke algebra of $\mathrm{G}\left(\mathbf{A}^{\mathrm{S}}\right)$. Then by the Fundamental Lemma $\left(f^{\mathrm{S}}\right)^{\vee} * \Phi \longleftrightarrow f_{\mathrm{S}}^{\prime} \otimes \mathrm{bc}\left(f^{\mathrm{S}}\right)$ where

$$
f^{\mathrm{S}} * \Phi(x)=\int_{\mathrm{G}\left(\mathbf{A}^{\mathrm{S}}\right)} f^{\mathrm{S}}(g) \Phi(x \bullet g) d g, \quad x \in \mathbf{X}_{\mathbf{A}} .
$$

We infer an identity

$$
\begin{aligned}
& \sum_{(\mathrm{L}, \pi)} c_{\mathrm{L}}^{\mathrm{M}} \int_{\mathrm{ia}_{(\mathrm{L}, \pi)}^{*}} \widehat{f^{\mathrm{S}}}(\mathrm{I}(\pi, \lambda)) \tilde{\mathrm{B}}(\Phi: \pi, \lambda) d \lambda \\
& =\sum_{\left(\mathrm{L}_{1}^{\prime}, \pi^{\prime}\right)} \int_{\mathrm{ia}_{\mathrm{L}_{1}^{\prime}}^{*}} \widehat{f^{\mathrm{S}}}\left(\mathrm{bc}\left(\mathrm{I}\left(\pi^{\prime}, \lambda^{\prime}\right)\right) \mathbf{B}\left(f^{\prime}: \pi^{\prime}, \lambda^{\prime}\right) d \lambda^{\prime}\right.
\end{aligned}
$$

where the sums are over $\pi$ (resp. $\pi^{\prime}$ ) which are unramified outside S. To separate the contribution of (L, $\pi$ ) and $\lambda$ we appeal to [LR00, Lemma 4] whose conditions are satisfied by the Jacquet-Shalika classification Theorem [JS81b, JS81a]. 


\section{Appendix D: Upper semicontinuity of multiplicity, by Erez Lapid and Omer Offen}

The following statement and proof are well known to experts. For convenience we include a simple proof which was suggested to us by Joseph Bernstein, Akshay Venkatesh and Nolan Wallach. We are very grateful to them for allowing us to include the proof here.

For now $\mathrm{G}$ is any $p$-adic group and $\mathrm{H}$ is any closed subgroup of $\mathrm{G}$.

Lemma D.1. - Let $\pi_{\lambda}$ be an analytic family of admissible representations of $\mathrm{G}$ on $\mathrm{V}$. Suppose that there exist $k$ meromorphic families of linear functionals $l_{\lambda}^{i}, i=1, \ldots, k$ on $\mathrm{V}$ which are $\mathrm{H}$-invariant and generically linearly independent. Then $\operatorname{dim} \operatorname{Hom}_{\mathrm{H}}\left(\pi_{\lambda}, 1\right) \geq k$ for all $\lambda$.

Proof. - Let $\mathrm{K}_{1} \supset \mathrm{K}_{2} \supset \cdots$ be a basis of compact open subgroups of $\mathrm{G}$ and let $\mathrm{V}_{n}=\mathrm{V}^{\mathrm{K}_{n}}$. There exists $\mathrm{N}$ such that the restrictions of $l_{\lambda}^{1}, \ldots, l_{\lambda}^{k}$ to $\mathrm{V}_{\mathrm{N}}$ are generically linearly independent. Suppose that $\lambda_{0}$ is given. By passing to a one-parameter family in general position through $\lambda_{0}$, we may assume that the set of parameters is the unit disc $\mathrm{D}=\{\lambda \in \mathbf{C}:|\lambda|<1\}$, that $\lambda_{0}=0$, and that the restrictions of $l_{\lambda}^{1}, \ldots, l_{\lambda}^{k}$ to $\mathrm{V}_{\mathrm{N}}$ are linearly independent for $\lambda \in \mathrm{D} \backslash\{0\}$. For a finite-dimensional vector space $\mathrm{W}$ over $\mathbf{C}$, let $\mathcal{G}_{k}(\mathrm{~W})$ denote the Grassmannian variety of $k$-dimensional vector subspaces of $\mathbf{W}$, which we view as a closed subvariety of the projective space of $\bigwedge^{k} \mathrm{~W}$ through the Plücker embedding $\iota_{\mathrm{W}}$. For any $n \geq \mathrm{N}$, let

$$
\mu_{n}=\min _{v_{1}, \ldots, v_{k} \in \mathrm{V}_{n}} \operatorname{ord}_{\lambda=0} \operatorname{det}\left(l_{\lambda}^{i}\left(v_{j}\right)\right)_{i, j=1, \ldots, k} \in \mathbf{N} .
$$

The map $\lambda \mapsto\left\langle l_{\lambda}^{1}, \ldots, l_{\lambda}^{k}\right\rangle$ defines a holomorphic curve $\mathrm{F}_{n}: \mathrm{D} \rightarrow \mathcal{G}_{k}\left(\mathrm{~V}_{n}^{*}\right)$ such that, if we fix a basis $v_{1}, \ldots, v_{d}$ of $\mathrm{V}_{n}$, the homogeneous coordinates of $\iota_{\mathrm{V}_{n}^{*}}\left(\mathrm{~F}_{n}(\lambda)\right)$ are $\lambda^{-\mu_{n}} \operatorname{det}\left(l_{\lambda}^{i}\left(v_{m_{j}}\right)\right)_{i, j=1, \ldots, k}, 1 \leq m_{1}<\cdots<m_{k} \leq d$. By enlarging $\mathrm{N}$ if necessary, we may assume that $\mu_{n}=\mu_{\mathrm{N}}$ for all $n \geq \mathrm{N}$. Then for any $n \geq m \geq \mathrm{N}$ and for all $\lambda \in \mathrm{D}$ (including 0 ), $\mathrm{F}_{m}(\lambda)$ is the image of $\mathrm{F}_{n}(\lambda)$ under the restriction map $\mathrm{V}_{n}^{*} \rightarrow \mathrm{V}_{m}^{*}$. Thus, for any $\lambda \in \mathrm{D}$ we get a $k$-dimensional subspace $\mathrm{F}(\lambda)$ of $\mathrm{V}^{*}$ whose image in $\mathrm{V}_{n}^{*}$ is $\mathrm{F}_{n}(\lambda)$ for all $n \geq \mathrm{N}$. Fix $h \in \mathrm{H}$ and $n \geq \mathrm{N}$. By assumption, we have $\left.l \circ \pi_{\lambda}(h)\right|_{\mathrm{V}_{n}}=\left.l\right|_{\mathrm{V}_{n}}$ for any $l \in \mathrm{F}_{n}(\lambda)$. This equality depends only on $\left.l\right|_{\mathrm{V}_{m}}$ provided that $\mathrm{K}_{m} \subseteq \mathrm{K}_{n} \cap h \mathrm{~K}_{n} h^{-1}$. Therefore, $\left.l \circ \pi_{\lambda}(h)\right|_{\mathrm{V}_{n}}=\left.l\right|_{\mathrm{V}_{n}}$ for any $l \in \mathrm{F}(\lambda)$. It follows that $l$ is $\mathrm{H}$-invariant for any $l \in \mathrm{F}(\lambda), \lambda \in \mathrm{D}$.

Consider now the Archimedean case and assume in addition that $\mathbf{G}$ is reductive and $\mathbf{H}$ is the fixed point subgroup of an involution of $\mathbf{G}$. Let $\mathrm{K}$ be a maximal compact subgroup of $\mathrm{G}$ such that $\mathrm{H} \cap \mathrm{K}$ is a maximal compact subgroup of $\mathrm{H}$. (This can always be arranged.) The proof above works for the underlying $(\mathfrak{g}, \mathrm{K})$-module of $\pi_{\lambda}$ (where we take $\mathrm{V}_{n}$ to be the sum of the isotypic components of the first $n$ irreducible representations of $\mathrm{K}$, ordered arbitrarily). It yields that $\operatorname{dim}_{(\mathfrak{h}, \mathrm{H} \cap \mathrm{K})}\left(\pi_{\lambda}, 1\right) \geq k$ for all $\lambda$. By the automatic continuity Theorem for H-invariant functionals [vdBD88, BD92] we get $\operatorname{dim} \operatorname{Hom}_{H}\left(\pi_{\lambda}, 1\right) \geq k$. Thus Lemma D. 1 holds in this case as well. We thank Eitan Sayag for pointing this out to us. 


\section{Index of symbols}

A, Sect. 0

$\eta$, Sect. 0

$\mathbf{G}^{\prime}, \mathbf{G}, \mathbf{X}$, Sect. 0

$\mathcal{P}_{x}$, Sect. 0

bc, Sects. 0, 3.2

$\psi^{\prime}, \psi$, Sect. 1.1

$x \bullet g$, Sect. 1.1

$\mathrm{Q}^{x}$, Sect. 1.1

$\mathfrak{w}(x)$, Sect. 1.1

$\mathrm{P}_{0}, \mathrm{M}_{0}, \mathrm{U}_{0}$, Sect. 1.1

$\mathrm{P}_{\kappa}, \mathrm{M}_{\kappa}, \mathrm{U}_{\kappa}$, Sect. 1.1

$\overleftarrow{\mathrm{P}}, \overleftarrow{\mathrm{M}}, \overleftarrow{\mathrm{U}}$, Sect. 1.1

$\overleftarrow{\kappa}$, Sect. 1.1

$\Delta_{0}^{\mathrm{M}}$, Sect. 1.1

W, Sect. 1.1

$w_{0}^{\mathrm{M}}$, Sect. 1.1

$\mathrm{W}^{\mathrm{M}}(\mathrm{L})$, Sect. 1.1

$w \kappa$, Sect. 1.1

$\delta_{\mathrm{Q}}$, Sect. 1.1

$\mathfrak{a}_{\mathrm{Q}}^{*}, \mathfrak{a}_{\mathrm{Q}}, \mathfrak{a}_{0}^{*}, \mathfrak{a}_{0}$, Sect. 1.1

H, Sect. 1.1

$\rho$, Sect. 1.1

$\left(\mathfrak{a}_{\mathrm{M}}^{*}\right)_{+}$, Sect. 1.1

$\mathbf{1}_{\Gamma}, 1_{\mathrm{Q}}$, Sect. 1.1

$\mathrm{L}(x), \mathrm{R}(y)$, Sect. 1.1

$\mathcal{S}(\mathrm{Y})$, Sect. 1.1

$f * \Phi$, Sect. 1.1

$\boldsymbol{\omega}_{\mathbf{v}}$, Sect. 1.2

$\mathcal{R}(\mathrm{G})$, Sect. 1.3

$\omega_{\pi}$, Sect. 1.3

$\pi^{*}, \pi^{\vee}$, Sect. 1.3

$\pi \cdot \chi, \pi[s], \pi^{J}$, Sect. 1.3

$\mathrm{I}_{\mathrm{M}}^{\mathrm{G}}(\sigma), \mathrm{I}_{\mathrm{M}}^{\mathrm{G}}(\sigma, \lambda)$, Sect. 1.3

$\mathrm{I}_{\mathrm{M}}^{\mathrm{G}}(\mathrm{T})$, Sect. 1.3

$\sigma_{1} \times \cdots \times \sigma_{t}$, Sect. 1.3

$\varphi \stackrel{\mu}{\leftrightarrows} \xi, \Gamma_{\mathrm{L}, \mathrm{M}}(\nu, \mu)$, Sect. 1.3

$\varphi_{\mathrm{M}}$, Sect. 1.3

$r_{\mathrm{L}, \mathrm{M}}, r_{\mathrm{M}}$, Sect. 1.3

$\psi_{0}^{\prime}, \psi_{0}$, Sect. 1.4

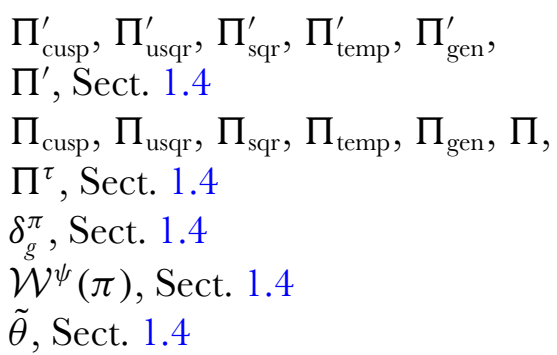

$\mathbf{W}_{e}(\varphi: \sigma, \lambda)$, Sects. 1.4, 11.1

$\mathbf{W}(\varphi, \sigma, \lambda)$, Sects. $1.4,11.1$

$w \sigma$, Sect. 1.4

$\overleftarrow{\sigma}, \overleftarrow{\lambda}$, Sect. 1.4

$\mathrm{M}(w, \sigma, \lambda), \mathrm{M}(w, \mathcal{W}(\sigma), \lambda)$, Sect. 1.4

$\mathcal{R}_{\text {pi }}(\mathrm{G})$, Sect. 1.4

$\mathrm{L}\left(s, \pi_{1} \times \pi_{2}\right), \varepsilon\left(s, \pi_{1} \times \pi_{2} ; \psi\right)$, Sect. 1.4

$\gamma\left(s, \pi_{1} \times \pi_{2} ; \psi\right)$, Sect. 1.4

$\mathrm{C}_{\mathrm{M}}(w: \sigma, \lambda ; \psi)$, Sect. 1.4

$\mathfrak{c s}(\sigma, \lambda)$, Sects. 1.4, 11.1

$\mathrm{N}(w, \sigma, \lambda), \mathrm{N}(w, \mathcal{W}(\sigma), \lambda)$, Sect. 1.4

$\operatorname{LQ}(\sigma, \lambda)$, Sect. 1.5

$\Pi_{\text {unr }}$, Sect. 1.5

$\hat{f}(\pi)$, Sect. 1.5

$\sigma[\mathbf{Z}]$, Sect. 1.5

$\operatorname{supp}_{c}(\pi)$, Sect. 1.5

$\unlhd, \triangleleft$, Sect. 1.5

$\mathfrak{B}_{\mathcal{D}}^{\ell, \hat{\ell}}(f)$, Sect. 2.1

$\mathcal{D}^{\circ}$, Sect. 2.1

$\mathcal{D} \cdot \chi$, Sect. 2.1

$\mathrm{I}(\mathcal{D}, \lambda)$, Sect. 2.1

$\|g\|$, Sect. 2.1

$\mathcal{E}_{\mathrm{G}}\left(\mathrm{X}, \pi^{*}\right)$, Sect. 2.1

$\Phi \odot \alpha$, Sect. 2.1

$\alpha \circ \mathrm{T}$, Sect. 2.1

$\mathfrak{W}(\pi)$, Sect. 2.2

$\mathbf{B}_{\pi^{\prime}}$, Sects. 2.2, 10.3

$\mathcal{S}^{*}\left(\mathrm{G}^{\prime}\right)^{\left({ }^{t} \mathrm{U}_{0}^{\prime} \times \mathrm{U}_{0}^{\prime},{ }^{t} \psi_{0}^{\prime} \times \psi_{0}^{\prime-1}\right)}$,

Sect. 2.2

$\mathbf{B}\left(f^{\prime}: \sigma^{\prime}, \lambda\right)$, Sect. 2.2

$\mathrm{G}^{\prime \pm}$, Sect. 2.2 
$\Phi \longleftrightarrow f^{\prime}, f \stackrel{x}{\longleftrightarrow} f^{\prime}$, Sect. 3.1

$\mathrm{X}[x], \mathrm{G}^{\prime}[x]$, Sect. 3.1

$\mathrm{D} \longleftrightarrow \mathrm{D}^{\prime}$, Sect. 3.1

$\mathcal{S}^{*}(\mathrm{X})^{\left(\mathrm{U}_{0}, \psi_{0}^{-1}\right)}$, Sect. 3.1

ai, Sect. 3.2

$\boldsymbol{\lambda}_{\psi^{\prime}}$, Sect. 3.2

$\mathcal{B}(\pi), \mathfrak{b}_{\pi},[\mathcal{B}](\pi),[\mathfrak{b}]_{\pi}$, Sect. 3.2

$\tilde{\mathfrak{w}}(\pi)$, Sect. 3.2

$\tilde{\mathfrak{w}}^{\prime}\left(\pi^{\prime}\right)$, Sect. 3.2

$\Pi^{\tau, \mathrm{ti}}, \Pi^{\tau, \text { an }}$, Sect. 3.2

$\Pi_{\mathrm{bc}-\text { gen }}^{\prime}$, Sect. 3.2

$\alpha^{\pi^{\prime}}, \mathcal{B I}_{x}, \mathcal{B I}$, Sect. 3.3

$\mathcal{B I}_{\mathrm{M}, x}, \mathcal{B I}_{\mathrm{M}}$, Sect. 3.3

$\mathbf{X}^{\circ}$, Sect. 4

$\mathrm{J}_{\mathrm{M}}(\varphi: x, \alpha, \lambda)$, Sect. 4

$\mathrm{G}^{\circ}[x]$, Sect. 4

$\Phi^{\mathrm{M}}$, Sect. 4

$\mathfrak{n}\left(\sigma^{\prime}, \lambda\right), \mathcal{J}_{\sigma^{\prime}}(x, \alpha, \lambda)$, Sect. 4

$\tilde{\mathbf{B}}\left(\sigma^{\prime}, \lambda\right)$, Sect. 4

$\mathbf{X}_{\mathbf{L}}$, Sect. 5.1

$\theta$, Sect. 5.1

$\left(\mathfrak{a}_{\mathrm{M}}^{*}\right)^{w}$, Sect. 5.1
$\mathrm{Z}(\varphi: x, \beta, \lambda)$, Sect. 5.1

$\mathcal{F}(\varphi: x, \beta, \lambda)$, Sect. 5.2

$\beta^{\varrho}$, Sect. 5.3

$\tilde{\mathbf{D}}_{\mathrm{L}}(\varrho, \lambda)$, Sect. 5.3

$\mathrm{W}_{2}[\mathrm{M}], \mathrm{M}(w)$, Sect. 6

$\mathfrak{I}, \prec$, Sect. 6

$\mathfrak{F}_{w}$, Sect. 6

$[\cdot, \cdot]_{\pi}$, Sect. A. 1

$\mathrm{A}_{\mathrm{G}}$, Sect. 10.1

$\mathcal{C}$, Sect. 10.3

$\mathfrak{A}_{\pi}$, Sect. 10.3

$\mathbf{W}^{\pi}, \mathbf{W}_{e}^{\pi}$, Sect. 10.3

$\mathfrak{r} \mathfrak{l}^{\prime \pi^{\prime}}$, Sect. 10.3

$\mathfrak{W}\left(\pi^{\prime}\right)$, Sect. 10.3

$\mathcal{A}_{\mathrm{M}, \sigma}$, Sect. 11.1

$\mathrm{E}(g, \varphi, \lambda)$, Sect. 11.1

$\mathfrak{A}(\sigma, \lambda)$, Sect. 11.1

$\Xi$, Sect. 11.2

$\mathfrak{r l}(\sigma, \lambda)$, Sect. 11.2

$\mathrm{J}(\varphi: x, \sigma, \lambda)$, Sect. 11.2

$\mathcal{C} \neg \tau$, Sect. 11.4

$\Pi_{\text {reg }}^{\tau}$, Sect. 13.2

\section{REFERENCES}

AC89. J. ARthur and L. Clozel, Simple Algebras, Base Change, and the Advanced Theory of the Trace Formula, Annals of Mathematics Studies, vol. 120, Princeton University Press, Princeton, 1989. MR 1007299 (90m:22041).

AG. A. Aizenbud and D. Gourevitch, Smooth transfer of Kloosterman integrals (the Archimedean case), Amer. f. Math., to appear.

AG08. A. Aizenbud and D. Gourevitch, Schwartz functions on Nash manifolds, Int. Math. Res. Not., 5 (2008), rnm155. 37. MR 2418286 (2010g:46124).

AG09a. A. Aizenbud and D. Gourevitch, Generalized Harish-Chandra descent, Gelfand pairs, and an Archimedean analog of Jacquet-Rallis's theorem, Duke Math. F., 149 (2009), 509-567, with an appendix by the authors and Eitan Sayag. MR 2553879 (201 lc:22026).

AG09b. A. Aizenbud and D. Gourevitch, Multiplicity one theorem for $\left(\mathrm{GL}_{n+1}(\mathbf{R}), \mathrm{GL}_{n}(\mathbf{R})\right)$, Sel. Math. New Ser., 15 (2009), 271-294. MR 2529937 (2010i:22012).

AG10. A. Aizenbud and D. Gourevitch, The de-Rham theorem and Shapiro lemma for Schwartz function on Nash manifolds, Isr. F. Math., 177 (2010), 155-188. MR 2684417.

AGS08. A. Aizenbud, D. Gourevitch, and E. SAyaG, $\left(\mathrm{GL}_{n+1}(\mathrm{~F}), \mathrm{GL}_{n}(\mathrm{~F})\right)$ is a Gelfand pair for any local field F, Compos. Math., 144 (2008), 1504-1524. MR 2474319 (2009k:22022).

Art78. J. G. ARTHUR, A trace formula for reductive groups. I. Terms associated to classes in G(Q), Duke Math. F., 45 (1978), 911-952. MR 518111 (80d:10043).

Art85. J. ARthur, A measure on the unipotent variety, Can. F. Math., 37 (1985), 1237-1274. MR 828844 (87m:22049).

Art86. J. ARThur, On a family of distributions obtained from orbits, Can. F. Math., 38 (1986), 179-214. MR 835041 (87k:11058). 
Bar89. D. Barbasch, The unitary dual for complex classical Lie groups, Invent. Math., 96 (1989), 103-176. MR 981739 (90c:22044).

Bar03. E. M. BARUch, A proof of Kirillov's conjecture, Ann. Math. (2), 158 (2003), 207-252. MR 1999922 (2004f:22012).

BD92. J.-L. BRYLinski and P. Delorme, Vecteurs distributions H-invariants pour les séries principales généralisées d'espaces symétriques réductifs et prolongement méromorphe d'intégrales d'Eisenstein, Invent. Math., 109 (1992), 619664. MR 1176208 (93m:22016).

BD08. P. Blanc and P. Delorme, Vecteurs distributions H-invariants de représentations induites, pour un espace symétrique réductif $p$-adique G/H, Ann. Inst. Fourier (Grenoble), 58 (2008), 213-261. MR 2401221 (2009e:22015).

Ber84. J. N. Bernstein, P-invariant distributions on GL(N) and the classification of unitary representations of GL(N) (non-Archimedean case), in Lie group representations, II. Lecture Notes in Math., vol. 1041, pp. 50-102, Springer, Berlin, 1984. MR 748505 (86b:22028).

BK. J. Bernstein and B. Krötz, Smooth Fréchet globalizations of Harish-Chandra modules, Israel f. Math., to appear.

BK93. G. J. Bushnell and P. C. Kutzko, The Admissible Dual of GL(N) via Compact Open Subgroups, Annals of Mathematics Studies, vol. 129, Princeton University Press, Princeton, 1993. MR 1204652 (94h:22007).

BZ76. I. N. BernšteǏn and A. V. ZeLEvinskiĬ, Representations of the group GL $(n, \mathrm{~F})$, where $\mathrm{F}$ is a local non-Archimedean field, Usp. Mat. Nauk, 31 (1976), 5-70. MR 0425030 (54 \#12988) no. 3(189).

BZ77. I. N. Bernstein and A. V. Zelevinsky, Induced representations of reductive p-adic groups. I, Ann. Sci. Éc. Norm. Super. (4), 10 (1977), 441-472. MR 0579172 (58 \#28310).

CD94. J. CARmona and P. Delorme, Base méromorphe de vecteurs distributions H-invariants pour les séries principales généralisées d'espaces symétriques réductifs: equation fonctionnelle, f. Funct. Anal., 122 (1994), 152-221. MR 1274587 (95g:22021).

Châ99. N. B. Châu, Le lemme fondamental de Jacquet et Ye en caractéristique positive, Duke Math. J., 96 (1999), 473-520. MR 1671212 (2000f:11059).

CO07. G. Chinta and O. OfFen, Unitary periods, Hermitian forms and points on flag varieties, Math. Ann., 339 (2007), 891-913. MR 2341906 (2009e:11070).

CS80. W. Gasselman and J. Shalika, The unramified principal series of $p$-adic groups. II. The Whittaker function, Compos. Math., 41 (1980), 207-231. MR 581582 (83i:22027).

CS98. W. Casselman and F. Shahidi, On irreducibility of standard modules for generic representations, Ann. Sci. Éc. Norm. Super. (4), 31 (1998), 561-589. MR 1634020 (99f:22028).

DKV84. P. Deligne, D. Kazhdan, and M.-F. Vignéras, Représentations des algèbres centrales simples p-adiques, in Representations of Reductive Groups over a Local Field, Travaux en Cours, pp. 33-117, Hermann, Paris, 1984. MR 771672 (86h:11044).

FJŌS88. M. Flensted-Jensen, T. Ōshima, and H. Schlichtkrull, Boundedness of certain unitarizable Harish-Chandra modules, in Representations of Lie groups, Kyoto, Hiroshima, 1986. Adv. Stud. Pure Math., vol. 14, pp. 651-660, Academic Press, Boston, 1988. MR 1039855 (91e:22015).

GK75. I. M. GEL'FAND and D. A. KaJDAn, Representations of the group GL $(n, \mathrm{~K})$ where K is a local field, in Lie Groups and Their Representations, pp. 95-118, Halsted, New York, 1975. MR 0404534 (53 \#8334).

Gow84. R. Gow, Two multiplicity-free permutation representations of the general linear group GL(n, $\left.q^{2}\right)$, Math. Z., 188 (1984), 45-54. MR 767361 (86a:20008).

Hen10. G. Henniart, Induction automorphe pour GL(n, G), J. Funct. Anal., 258 (2010), 3082-3096. MR 2595735 (2011e:22028).

HH95. G. Henniart and R. Herb, Automorphic induction for GL(n) (over local non-Archimedean fields), Duke Math. F., 78 (1995), 131-192. MR 1328755 (96i:22038).

Hir99. Y. HironaKa, Spherical functions and local densities on Hermitian forms, J. Math. Soc. Jpn., 51 (1999), 553-581. MR 1691493 (2000c:11064).

HLR86. G. Harder, R. P. Langlands, and M. Rapoport, Algebraische Zyklen auf Hilbert-Blumenthal-Flächen, f. Reine Angew. Math., 366 (1986), 53-120. MR 833013 (87k:11066).

HM98. J. Hakim and Z. Mao, Supercuspidal representations of GL(n) distinguished by a unitary subgroup, Pac. F. Math., 185 (1998), 149-162. MR 1653208 (99j:22023).

HM02a. J. HAKim and F. Murnaghan, Globalization of distinguished supercuspidal representations of GL(n), Can. Math. Bull., 45 (2002), 220-230. MR 1904086 (2003f:22022).

HM02b. J. Hakim and F. Murnaghan, Tame supercuspidal representations of GL( $n)$ distinguished by a unitary group, Compos. Math., 133 (2002), 199-244. MR 1923582 (2003g:22019).

HW93. A. G. Helminck and S. P. WAng, On rationality properties of involutions of reductive groups, Adv. Math., 99 (1993), 26-96. MR 1215304 (94d:20051). 
Ich08. A. Ichino, Trilinear forms and the central values of triple product L-functions, Duke Math. F., 145 (2008), $281-307$. MR 2449948 (2009i:1 1066).

II10. A. IChINO and T. IKEDA, On the periods of automorphic forms on special orthogonal groups and the Gross-Prasad conjecture, Geom. Funct. Anal., 19 (2010), 1378-1425. MR 2585578.

Jac67. H. Jacquet, Fonctions de Whittaker associées aux groupes de Chevalley, Bull. Soc. Math. Fr., 95 (1967), $243-309$. MR 0271275 (42 \#6158).

Jac92. H. JacQuet, Relative Kloosterman integrals for GL(3). II, Can. F. Math., 44 (1992), 1220-1240. MR 1192415 (94c:11048).

Jac95. H. JACQUET, The continuous spectrum of the relative trace formula for GL(3) over a quadratic extension, Isr. $\mathcal{F}$. Math., 89 (1995), 1-59. MR 1324453 (96a:22029).

Jac98. H. JACQuet, A theorem of density for Kloosterman integrals, Asian f. Math., 2 (1998), 759-778. Mikio Sato: a great Japanese mathematician of the twentieth century. MR 1734128 (2001e:11054).

Jac01. H. JaCQuet, Factorization of period integrals, f. Number Theory, 87 (2001), 109-143. MR 1816039 (2002a:1 1050).

Jac02. H. Jacouet, Transfert lisse d'intégrales de Kloosterman, C. R. Math. Acad. Sci. Paris, 335 (2002), 229-232. MR 1933663 (2003k:1 1082).

Jac03a. H. JacQuet, Facteurs de transfert pour les intégrales de Kloosterman, C. R. Math. Acad. Sci. Paris, 336 (2003), 121-124. MR 1969564 (2004e:11052).

Jac03b. H. JacQuet, Smooth transfer of Kloosterman integrals, Duke Math. J., 120 (2003), 121-152. MR 2010736 (2005a:1 1066).

Jac04a. H.JAcQuet, Integral representation of Whittaker functions, in Contributions to Automorphic Forms, Geometry, and Number Theory, pp. 373-419, Johns Hopkins Univ. Press, Baltimore, 2004. MR 2058615 (2005f:11100).

Jac04b. H. JACQuet, Kloosterman identities over a quadratic extension, Ann. Math. (2), 160 (2004), 755-779. MR 2123938 (2006d:11051).

Jac05a. H. Jacquet, Kloosterman identities over a quadratic extension. II, Ann. Sci. Éc. Norm. Super. (4), 38 (2005), 609 669. MR 2172953 (2006j:11070).

Jac05b. H. Jacquet, Kloosterman integrals for GL(2, R), Pure Appl. Math. Q., 1 (2005), 257-289. MR 2194725 (2007j:22019) no. 2, part 1.

Jac09. H. JACQUET, Archimedean Rankin-Selberg integrals, in Automorphic Forms and L-functions II. Local Aspects, Contemp. Math., vol. 489, pp. 57-172, Amer. Math. Soc., Providence, 2009. MR 2533003 (2011a:11103).

Jac10. H. JAcquet, Distinction by the quasi-split unitary group, Isr. F. Math., 178 (2010), 269-324. MR 2733072 (2011k:11073).

JLR99. H. Jacquet, E. LAPID, and J. Rogawski, Periods of automorphic forms, 7. Am. Math. Soc., 12 (1999), 173-240. MR 1625060 (99c:11056).

JLR04. H. Jacouet, E. LAPID, and S. Rallis, A spectral identity for skew symmetric matrices, in Contributions to Automorphic Forms, Geometry, and Number Theory, pp. 421-455, Johns Hopkins Univ. Press, Baltimore, 2004. MR 2058616 (2005h:11105).

JPSS83. H. Jacquet, I. I. Piatetskit-Shapiro, and J. A. Shalika, Rankin-Selberg convolutions, Am. F. Math., 105 (1983), 367-464. MR 701565 (85g:11044).

JS81a. H. Jacquet and J. A. Shalika, On Euler products and the classification of automorphic forms. II, Am. F. Math., 103 (1981), 777-815. MR 623137 (82m:10050b).

JS81b. H. JACQUET and J. A. Shalika, On Euler products and the classification of automorphic representations. I, Am. $\mathcal{F}$. Math., 103 (1981), 499-558. MR 618323 (82m:10050a).

JS83. H. Jacquet and J. Shalika, The Whittaker models of induced representations, Pac. F. Math., 109 (1983), $107-120$. MR $716292(85 \mathrm{~h}: 22023)$.

JS90. H. JACQUET and J. Shalika, Rankin-Selberg convolutions: Archimedean theory, in Festschrift in Honor of I. I. PiatetskiShapiro on the Occasion of His Sixtieth Birthday, Part I. Israel Math. Conf. Proc. (Ramat Aviv, 1989), vol. 2, pp. 125-207, Weizmann, Jerusalem, 1990. MR 1159102 (93d:22022).

JY90. H. Jacquet and Y. Ye, Une remarque sur le changement de base quadratique, C. R. Acad. Sci. Paris Sér. I Math., 311 (1990), 671-676. MR 1081622 (92j:11046).

JY92. H. JACQUET and Y. Ye, Relative Kloosterman integrals for GL(3), Bull. Soc. Math. Fr., 120 (1992), 263-295. MR 1180831 (94c:11047).

JY96. H. JACQUeT and Y. Ye, Distinguished representations and quadratic base change for GL(3), Trans. Am. Math. Soc., 348 (1996), 913-939. MR 1340178 (96h:11041).

JY99. H. JAcQUET and Y. Ye, Germs of Kloosterman integrals for GL(3), Trans. Am. Math. Soc., 351 (1999), $1227-1255$. MR 1443878 (99j:11053). 
Lag08. N. Lagier, Terme constant de fonctions sur un espace symétrique réductif p-adique, f. Funct. Anal., 254 (2008), 1088-1145. MR 2381204 (2009d:22013).

Lap06. E. M. LAPID, On the fine spectral expansion of Jacquet's relative trace formula, f. Inst. Math. Fussieu, 5 (2006), 263-308. MR 2225043 (2007d:11059).

Lap08. E. M. LAPID, A remark on Eisenstein series, in Eisenstein Series and Applications. Progr. Math., vol. 258, pp. 239-249, Birkhäuser, Boston, 2008. MR 2402686 (2009m:11072).

LL79. J.-P. Labesse and R. P. LANGLANDS, L-indistinguishability for SL(2), Can. F. Math., 31 (1979), 726-785. MR 540902 (81b:22017).

LM. E. Lapid and A. Mínguez, On a determinantal formula of Tadić, Amer. F. Math., to appear.

LM09a. E. LAPID and Z. MAO, On the asymptotics of Whittaker functions, Represent. Theory, 13 (2009), 63-81. MR 2495561 (2010b:22024).

LM09b. E. LAPID and W. MülLER, Spectral asymptotics for arithmetic quotients of SL(n, R)/SO(n), Duke Math. F., 149 (2009), 117-155. MR 2541128.

LO07. E. Lapid and O. Offen, Compact unitary periods, Compos. Math., 143 (2007), 323-338. MR 2309989 (2008g:11091).

LR00. E. LaPID and J. Rogawski, Stabilization of periods of Eisenstein series and Bessel distributions on GL(3) relative to U(3), Doc. Math., 5 (2000), 317-350 (electronic). MR 1767567 (2002b:11068).

LR01. E. Lapid and J. Rogawski, Periods of Eisenstein series, C. R. Acad. Sci. Paris Sér. I Math., 333 (2001), 513-516. MR 1860921 (2002k:11072).

LR03. E. M. Lapid and J. D. Rogawski, Periods of Eisenstein series: the Galois case, Duke Math. J., 120 (2003), $153-226$. MR 2010737 (2004m:11077).

MW86. G. Møglin and J.-L. Waldspurger, Sur l'involution de Zelevinski, f. Reine Anger. Math., 372 (1986), $136-177$. MR 863522 (88c:22019).

MW89. C. Meglin and J.-L. Waldspurger, Le spectre résiduel de GL(n), Ann. Sci. Éc. Norm. Super. (4), 22 (1989), 605674. MR 1026752 (91b:22028).

Ngô99. B. C. NGÔ, Faisceaux pervers, homomorphisme de changement de base et lemme fondamental de Jacquet et Ye, Ann. Sci. Éc. Norm. Super. (4), 32 (1999), 619-679. MR 1710755 (2001g:11076).

Off07. O. Offen, Stable relative Bessel distributions on GL(n) over a quadratic extension, Am. J. Math., 129 (2007), 1183-1226. MR 2354318 (2009j:22021).

Off09. O. OfFen, Unitary periods and Jacquet's relative trace formula, in Automorphic Forms and L-functions I. Global Aspects, Contemp. Math., vol. 488, pp. 183-236, Amer. Math. Soc., Providence, 2009. MR 2522031 (2010k:11083).

Pou72. N. Skovhus Poulsen, On $\mathrm{C}^{\infty}$-vectors and intertwining bilinear forms for representations of Lie groups, f. Funct. Anal., 9 (1972), 87-120. MR 0310137 (46 \#9239).

Pra01. D. Prasad, On a conjecture of Jacquet about distinguished representations of GL(n), Duke Math. 7., 109 (2001), 67-78. MR 1844204 (2002g:22036).

Sar04. P. SARnaK, A letter to Cathleen Morawetz, http://www.math.princeton.edu/sarnak (2004).

Sha84. F. SHAHId, Fourier transforms of intertwining operators and Plancherel measures for GL(n), Am. F. Math., 106 (1984), 67-111. MR 729755 (86b:22031).

Sha85. F. SHaHidi, Local coefficients as Artin factors for real groups, Duke Math. J., 52 (1985), 973-1007. MR 816396 (87m:11049).

Sha90. F. SHAHIDI, A proof of Langlands' conjecture on Plancherel measures; complementary series for $p$-adic groups, Ann. Math. (2), 132 (1990), 273-330. MR 1070599 (91m:11095).

Shi76. T. Shintani, Two remarks on irreducible characters of finite general linear groups, f. Math. Soc. Jpn., 28 (1976), 396-414. MR 0414730 (54 \#2825).

Spr85. T. A. Springer, Some results on algebraic groups with involutions, in Algebraic Groups and Related Topics. Adv. Stud. Pure Math., vol. 6, pp. 525-543, North-Holland, Amsterdam, 1985. MR 803346 (86m:20050).

Tad86. M. TAdić, Classification of unitary representations in irreducible representations of general linear group (nonArchimedean case), Ann. Sci. Éc. Norm. Super. (4), 19 (1986), 335-382. MR 870688 (88b:22021).

vdBD88. E. van den BAN and P. Delorme, Quelques propriétés des représentations sphériques pour les espaces symétriques réductifs, f. Funct. Anal., 80 (1988), 284-307. MR 961900 (89j:22025).

Wal85. J.-L. WaLdspurger, Sur les valeurs de certaines fonctions L automorphes en leur centre de symétrie, Compos. Math., 54 (1985), 173-242. MR 783511 (87g:11061b).

Wal92. N. R. Wallach, Real Reductive Groups. II. Pure and Applied Mathematics, vol. 132, Academic Press, Boston, 1992. MR 1170566 (93m:22018). 
Wei82. A. WeIL, Adeles and Algebraic Groups. Progress in Mathematics, vol. 23, Birkhäuser, Boston, 1982. With appendices by M. Demazure and Takashi Ono. MR 670072 (83m:10032).

Ye88. Y. YE, Kloosterman integrals and base change, in Number Theory and Its Applications in China. Contemp. Math., vol. 77, pp. 163-170, Amer. Math. Soc., Providence, 1988. MR 973234 (90b:11058).

Ye89. Y. Ye, Kloosterman integrals and base change for GL(2), f. Reine Angew. Math., 400 (1989), 57-121. MR 1013725 (90i:11134).

Ye93. Y. YE, The fundamental lemma of a relative trace formula for GL(3), Compos. Math., 89 (1993), 121-162. MR 1255692 (95b:22023).

Ye94. Y. Ye, An integral transform and its applications, Math. Ann., 300 (1994), 405-417. MR 1304430 (95j:1 1045).

Ye95. Y. YE, The lifting of Kloosterman sums, F. Number Theory, 51 (1995), 275-287. MR 1326749 (97a:11126).

Ye98. Y. YE, A Kloosterman sum in a relative trace formula for $\mathrm{GL}_{4}$, Represent. Theory, 2 (1998), 370-392 (electronic). MR 1641835 (99j:11092).

Zel80. A. V. Zelevinsky, Induced representations of reductive p-adic groups. II. On irreducible representations of GL $(n)$, Ann. Sci. Éc. Norm. Super. (4), 13 (1980), 165-210. MR 584084 (83g:22012).

\section{B. Feigon}

Department of Mathematics, The City College of New York, New York, NY 10031, USA

bfeigon@ccny.cuny.edu

\section{E. Lapid}

Institute of Mathematics,

The Hebrew University of Jerusalem, Jerusalem 91904, Israel

erezla@math.huji.ac.il

\section{O. Offen}

Department of Mathematics,

Technion-Israel Institute of Technology,

Haifa 32000, Israel

offen@tx.technion.ac.il
Manuscrit reçu le 10 mars 2011

Manuscrit accepté le 28 avril 2012 publié en ligne le 1 juin 2012. 